\title{
WestVirginiaUniversity
}

THE RESEARCH REPOSITORY @ WVU

Graduate Theses, Dissertations, and Problem Reports

2008

\section{Electrical and optical properties of zinc oxide for scintillator applications}

Xiaocheng Yang
West Virginia University

Follow this and additional works at: https://researchrepository.wvu.edu/etd

\section{Recommended Citation}

Yang, Xiaocheng, "Electrical and optical properties of zinc oxide for scintillator applications" (2008). Graduate Theses, Dissertations, and Problem Reports. 2729.

https://researchrepository.wvu.edu/etd/2729

This Dissertation is protected by copyright and/or related rights. It has been brought to you by the The Research Repository @ WVU with permission from the rights-holder(s). You are free to use this Dissertation in any way that is permitted by the copyright and related rights legislation that applies to your use. For other uses you must obtain permission from the rights-holder(s) directly, unless additional rights are indicated by a Creative Commons license in the record and/ or on the work itself. This Dissertation has been accepted for inclusion in WVU Graduate Theses, Dissertations, and Problem Reports collection by an authorized administrator of The Research Repository @ WVU.

For more information, please contact researchrepository@mail.wvu.edu. 


\title{
ELECTRICAL AND OPTICAL PROPERTIES OF ZINC OXIDE FOR SCINTILLATOR APPLICATIONS
}

\author{
By
}

\author{
Xiaocheng Yang \\ Dissertation submitted to the \\ Eberly College of Arts and Sciences \\ at West Virginia University \\ in partial fulfillment of the requirements \\ for the degree of \\ Doctor of Philosophy \\ in \\ Physics \\ Nancy C. Giles, Ph.D., Chair \\ Larry E. Halliburton, Ph.D. \\ Wathiq Abdul-Razzaq, Ph.D. \\ Feruz Ganikhanov, Ph.D. \\ Charter D. Stinespring, Ph. D. \\ Department of Physics \\ Morgantown, West Virginia \\ 2008
}

Keywords: Zinc Oxide, Photoluminescence, Hall Effect, Optical absorption Copyright 2008 Xiaocheng Yang 


\title{
Abstract \\ ELECTRICAL AND OPTICAL PROPERTIES OF ZINC OXIDE FOR SCINTILLATOR APPLICATIONS
}

\begin{abstract}
Xiaocheng Yang
Zinc oxide ( $\mathrm{ZnO})$ is a wide-band-gap semiconductor suitable for many optical and optoelectronic applications. Among these is to use single crystal, powder, or ceramic forms of $\mathrm{ZnO}$ as a fast $\mathrm{UV}$ scintillator. In this work, the electrical and optical properties of $\mathrm{ZnO}$ were studied using photoluminescence, $\mathrm{X}$-ray-induced luminescence, optical absorption, and Hall Effect techniques. This study included single crystal $\mathrm{ZnO}$ and $\mathrm{ZnO}$ :Ga samples grown from high-pressure-melt (HPM), seeded chemical-vapor-transport (SCVT), and hydrothermal (HYD) techniques; powder samples synthesized using both solution and solid-state processes, and purchased from different commercial sources; and ceramic samples prepared by hot-uniaxial-pressing and spark-plasma-sintering methods. Temperature-dependent PL and Hall measurements were combined to establish the luminescence origins in the n-type $\mathrm{ZnO}$ and ZnO:Ga single crystals. Based on a PL line-shape analysis, including band-gap renormalization, the direct (e,h) transition is the main luminescent channel in highly n-type $\mathrm{ZnO}: \mathrm{Ga}$, while FX and FX-LO recombinations are responsible for the UV PL from as-grown $\mathrm{ZnO}$. An intrinsic mobility limit for $\mathrm{n}$-type $\mathrm{ZnO}$ was established by including three major phononscattering mechanisms. Analysis of Hall data from single-crystal samples including both neutral- and ionized-impurity scatterings provided donor and acceptor concentrations and energy levels. High n-type single-crystal ZnO samples prepared either by Ga doping and codoping, or by after-growth treatments, were also studied. Absorption and reflectance data were used to obtain free carrier concentrations from the Ga-doped and co-doped crystals, and it was found that several samples with $\mathrm{n} \sim$ high- $10^{18}$ to low- $10^{19} \mathrm{~cm}^{-3}$ had optimum UV luminescence. Anneal treatments in reducing atmospheres increased free carrier concentrations in HPM and HYD samples, but an induced absorption band due to oxygen vacancies limited the UV emission from these samples. PL and X-ray-induced luminescence studies on powder ZnO:Ga samples demonstrated that high $\mathrm{Ga}$-doping levels and $\mathrm{H}$-anneal treatments can improve UV emission, while impurities such as $\mathrm{Cu}$ and $\mathrm{Li}$ enhance the lower energy visible emissions and affect the UV output. For ceramic forms of $\mathrm{ZnO}$, reduction of scattering losses remains as the main challenge for improved scintillation.
\end{abstract}




\section{ACKNOWLEDGMENTS}

I really couldn't find a right word to express my gratitude to Dr. Nancy Giles, my advisor. I would like to thank her for her support and guidance for my study and research here at her lab, but what I appreciate more is her exemplary dedication to research and teaching. Working with Dr. Giles, I learned not only that I can do research, but I can enjoy it, not only how to focus on the present project, but how to see the whole forest. Each time we talked about science, I benefited from her insightful and inspiring discussions. At times that I experienced personal frustrations, she always encouraged me to chin up again.

Thanks are also due to Dr. Larry Halliburton, for his help and encouragement on my study, and for his willingness to share his knowledge as well as his optimism and wisdom.

I'd like to thank my committee members Dr. Wathiq Abdul-Razzaq, Dr. Feruz Ganikhanov, and Dr. Charter Stinespring for sacrificing their priceless time to serve in my committee.

I would also like to acknowledge the support of Dr. John Neal at ORNL for his support, and thank our colleagues at ORNL and UC-Davis: Dr. Lynn Boatner, Dr. Zuhair Munir, Dr. David DeVito, Dr. Mei Hong, Beth Armstrong, Joanne Ramey, Banu Kesanli, and Jane Howe, for allowing me to be a member of a strong and fruitful team.

I appreciate the help and friendship I received from Shan Yang, Dr. Lihua Bai, Dr. Ming Luo, Dr. Chunchuan Xu, Dr. Yongquan Jiang, and Dr. Sean Evans.

Last but not least, I'd like to thank my wife Daisy for her support and understanding over these years, thank my son for his cheers, and thank my mother and my siblings for their encouragement from the other side of the globe. 


\section{TABLE OF CONTENTS}

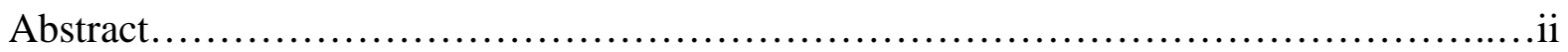

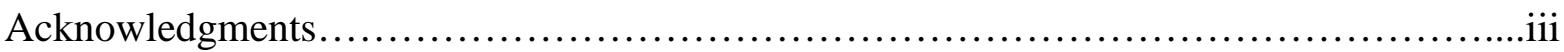

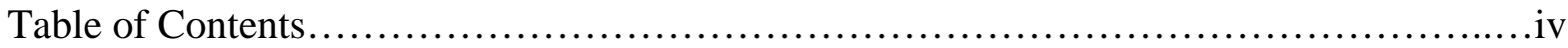

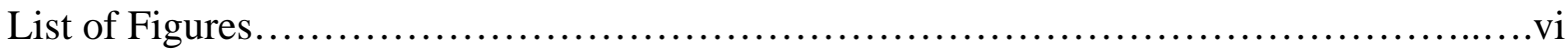

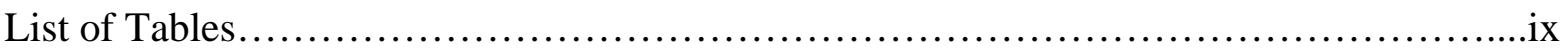

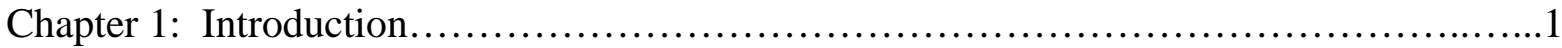

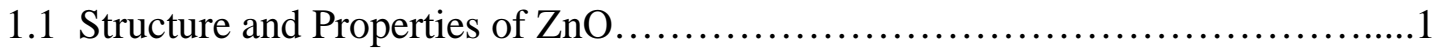

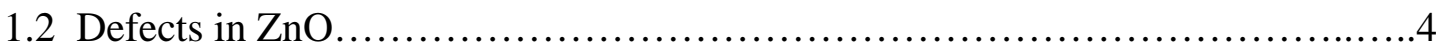

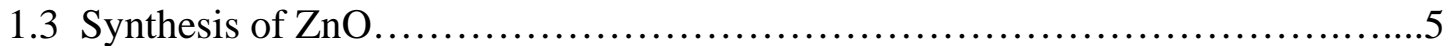

$1.4 \mathrm{ZnO}$ Applications: Scintillator and More.......................................

1.5 Motivation and Goals.........................................................13

Chapter 2: Photoluminescence and Hall Effect........................................ 15

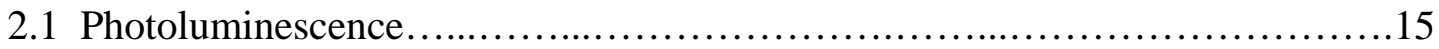

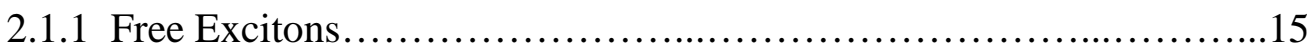

2.1.2 Bound Excitons .................................................20

2.1.3 Band-to-band Transitions..........................................22

2.1.4 Band-to-bound Transitions.....................................22

2.1.5 Donor-acceptor-pair Transitions....................................23

2.1.6 Competition between PL and Non-radiative Processes..................25

2.2 Temperature-dependent Bandgap and Bandgap Renormalization................26

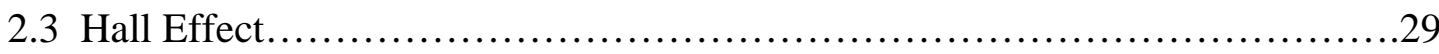

2.3.1 Hall r Factor..................................................... 31

2.3.2 Hall Measurements Using the van der Pauw Method....................34

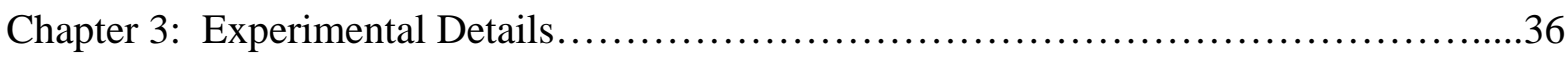

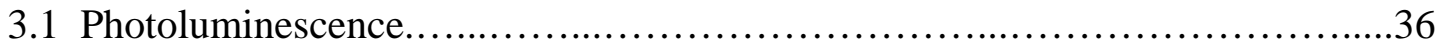

3.2 X-ray-induced Luminescence..............................................40

3.3 Hall Effect.............................................................. 40

3.4 Absorption................................................................... 
Chapter 4: Photoluminescence from As-grown and Gallium-doped ZnO.

4.1 Room Temperature Photoluminescence: Recombination Mechanisms..........44

4.2 Temperature-dependent Photoluminescence................................53

4.3 Effect of Self-absorption on Photoluminescence in Transmission

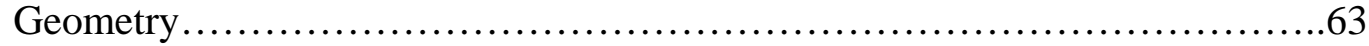

Chapter 5: Temperature-Dependent Hall Data Analysis..............................69

5.1 Hall Effect Analysis: General Approach....................................69

5.2 Intrinsic Hall r Factor and Intrinsic Mobility in $\mathrm{ZnO} \ldots \ldots \ldots \ldots \ldots \ldots \ldots \ldots \ldots . \ldots \ldots$

5.3 Impurity Scattering and Mobility Fitting................................. 80

5.4 Results and Discussion................................................ 84

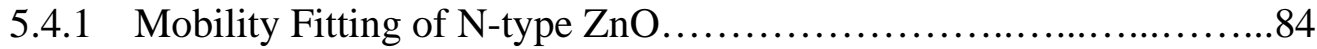

5.4 .2 Total Hall $r$ Factor............................................... 88

5.4.3 Free-carrier Concentration Fitting..............................89

5.5 Identification of Impurities............................................91

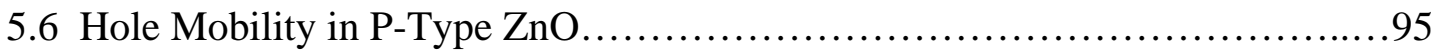

Chapter 6: Effects of Doping and Post-Growth Treatments on Optical and

Electrical Properties of Single-Crystal ZnO.............................100

6.1 Effects of Heavy Doping on ZnO.....................................101

6.2 Free Carrier Concentration Determined Using Infrared Reflectance

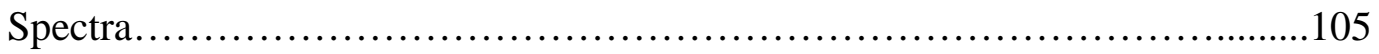

6.3 Effects of Anneal Treatments on $\mathrm{ZnO}$.....................................107

Chapter 7: Evaluation of Powder and Ceramic ZnO for Scintillator Applications..........116

7.1 PL Characterization of ZnO:Ga Powder Samples Prepared at ORNL...........117

7.2 PL Characterization of Commercial ZnO Powder Samples....................127

7.3 PL Characterization of Ceramic ZnO Samples............................130

7.4 X-ray-induced Luminescence of $\mathrm{ZnO}$ and $\mathrm{ZnO}$ :Ga Samples..................139

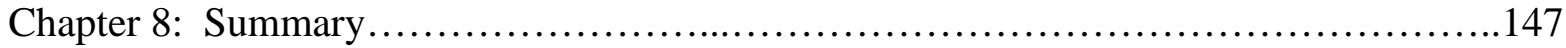

References.................................................................... 152

Curriculum Vitae..........................................................159 


\section{LIST OF FIGURES}

Fig. 1.1

Fig. 1.2

Fig. 1.3

Fig. 1.4

Fig. 1.5

Fig. 2.1

Fig. 2.2

Fig. 2.3

Fig. 2.4

Fig. 3.1

Fig. 3.2

Fig. 3.3

Fig. 4.1

Fig. 4.2

Fig. 4.3

Fig. 4.4

Fig. 4.5

Fig. 4.6

Fig. 4.7

Fig. 4.8

Fig. 4.9

Fig. 4.10

Fig. 4.11

Fig. 4.12

Fig. 4.13

Fig. 4.14

Fig. 4.15

Fig. 4.16

Fig. 4.17

ZnO crystal structures

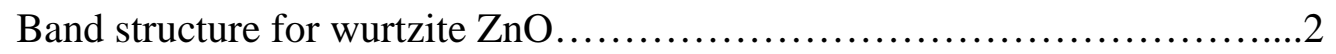

The high-pressure-melt growth apparatus.............................6

Effect of correction for sample self-absorption.........................11

The D-T neutron generator and associated $\alpha$-particle detector...............13

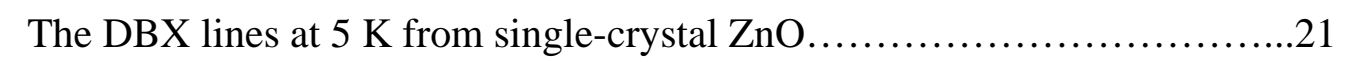

The DAP peak at $300 \mathrm{~K}$ from single-crystal $\mathrm{ZnO} . . . \ldots \ldots \ldots \ldots \ldots \ldots . . . . . . . .24$

A schematic sketch of a Hall effect measurement........................30

A schematic sketch of a van der Pauw Hall effect measurement.............34

PL setup for above-bandgap excitation experiments.......................36

PL setup for selective excitation experiments...............................39

A schematic sketch of X-ray excited luminescence experiment..............40

RT PL from undoped and Ga-doped Cermet ZnO.......................45

Schematic sketches of 300-K PL compared to the predicted bandgaps..........47

300-K PL from Cermet undoped $\mathrm{ZnO}$ and a theoretic fit..................48

RT PL from Cermet ZnO:Ga and a fit using indirect (e,h) model...........49

RT PL from Cermet ZnO:Ga and a fit using direct (e,h) model...............51

RT PL from Cermet ZnO:Ga using selected excitations.....................52

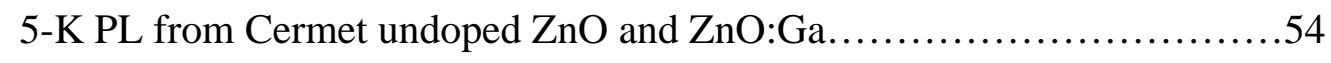

An expanded view of Fig. 4.7 in the near-band-edge region...................54

Temperature-dependent PL from Cermet undoped ZnO (5-78 K)............56

Temperature-dependent PL from Cermet undoped ZnO (78-300 K).........56

Temperature-dependent PL peak positions for Cermet undoped ZnO.........59

Temperature-dependent PL from Cermet ZnO:Ga (5-300 K).................60

Integrated PL intensities from Cermet undoped $\mathrm{ZnO}$ and $\mathrm{ZnO}: \mathrm{Ga} . . . . . . . . . . . .61$

A fit of FX transition energy using MW equation........................63

Correction for self-absorption effect for sample EP-14....................64

Correction for self-absorption effect for sample CMT1...................66

Correction for self-absorption effect for sample Cermet $\mathrm{ZnO}$ Ga.............67 
Fig. $5.1 \quad$ F-values for different donor levels.................................... 72

Fig. 5.2 Schematic diagrams of electron scattering by phonons......................78

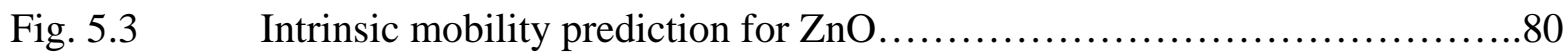

Fig. 5.4 A schematic sketch of electron scattering by an ionized impurity.............81

Fig. 5.5 Temperature-dependent $\mu$ data and fittings for four $\mathrm{ZnO}$ samples.............86

Fig. 5.6 Temperature-dependent total Hall $\mathrm{r}$ factors for four $\mathrm{ZnO}$ samples................89

Fig. 5.7 Temperature-dependent $\mathrm{n}$ data and fittings for four $\mathrm{ZnO}$ samples............91

Fig. 5.8 5-K PL edge emissions from samples EP and Cermet......................92

Fig. 5.9 DAP emission from sample TD (5-60 K) ...............................94

Fig. $5.10 \quad \mathrm{LiOH}^{-}$absorption at $10 \mathrm{~K}$ from sample TD.................................94

Fig. 5.11 Simulation of hole mobility in p-type $\mathrm{ZnO}\left(300-\mathrm{K} \mu=35 \mathrm{~cm}^{2} / \mathrm{Vs}\right) \ldots \ldots \ldots \ldots \ldots . . .97$

Fig. 5.12 Simulated Hall $\mu$ of holes as a function of compensation.....................98

Fig. 5.13 Simulation of hole mobility in p-type $\mathrm{ZnO}\left(300-\mathrm{K} \mu=2 \mathrm{~cm}^{2} / \mathrm{Vs}\right) \ldots \ldots \ldots \ldots \ldots . . .99$

Fig. 6.1 Absorption spectra from four as-grown single-crystal $\mathrm{ZnO}$............................100

Fig. $6.2 \quad$ Absorption spectra from as-grown and doped $\mathrm{ZnO}$.........................102

Fig. 6.3 Absorption spectra from doped and co-doped $\mathrm{ZnO}$ in log-log scale.............104

Fig. 6.4 FTIR free carrier absorption from two Cermet $\mathrm{ZnO}$ in log-log scale.........105

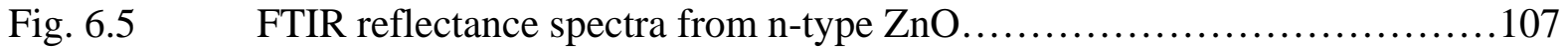

Fig. 6.6 Absorption spectra from heat-treated Cermet HPM ZnO......................108

Fig. $6.7 \quad 200-K$ PL from three HPM grown samples..............................110

Fig. 6.8 Absorption spectra from heat-treated Airtron HYD ZnO....................112

Fig. 6.9 Gaussian fit to absorption band at $3.03 \mathrm{eV}$ from heat-treated $\mathrm{ZnO}$............113

Fig. 7.1 300-K PL from JNA powder samples before and after $\mathrm{H}$-anneal..............120

Fig. 7.2 300-K PL from JNR powder samples before and after H-anneal..............120

Fig. 7.3 5-K PL from sample JNR-2 and a three-component fit........................121

Fig. 7.4 300-K PL from JNK powder samples before and after H-anneal.............123

Fig. 7.5 Comparison of the effects of $\mathrm{H}$ and Air anneals for sample JNK-6.............124

Fig. 7.6 TEM images of sample JNK-8 before and after $\mathrm{H}$-anneal..................125

Fig. $7.7 \quad 300-K$ PL from JNK-8 and JNC powders................................126

Fig. 7.8 300-K PL from commercial AA and SA ZnO powders....................128

Fig. 7.9 300-K and 5-K PL from AA ZnO powders of different impurities............129 
Fig. 7.10 300-K PL from HUP sintered ZnO ceramics...........................131

Fig. 7.11 300-K PL from SPS sintered ZnO ceramics...........................133

Fig. 7.12 80-K PL from SPS ceramic Pure950 after annealed in air and LiF.............134

Fig. 7.13 80-K PL from two SPS ceramics after anneal in air.........................135

Fig. 7.14 300-K PL from powder and ceramic WL1201..........................137

Fig. 7.15 300-K PL from ceramic BK5-203 before and after $\mathrm{H}$ anneal.................138

Fig. 7.16 300-K PL from SPS ceramic AA23558 before and after $\mathrm{H}$ anneal...........139

Fig. 7.17 PL and X-ray-induced luminescence from Cermet ZnO:Ga.................140

Fig. $7.18 \quad \mathrm{X}$-ray-induced luminescence: reflection vs transmission.................141

Fig. 7.19 PL and X-ray-induced luminescence from AA and SA powder ZnO.......143

Fig. 7.20 PL and X-ray-induced luminescence from four ZnO:Ga powders..........144

Fig. 7.21 X-ray-induced luminescence from SPS ceramic Pure950..................145

Fig. $7.22 \quad$ X-ray-induced luminescence from ceramic WL1201.....................146 


\section{LIST OF TABLES}

Table 1.1 Structure and electronic parameters of wurtzite $\mathrm{ZnO}$.

Table 5.1 Parameters for $\mu$ and $n$ fittings for four n-type single-crystal $\mathrm{ZnO}$..........90

Table 6.1 Free carrier concentration and p-value obtained from absorption data.........104

Table 6.2 Free carrier concentration from Hall measurements and comparison of free carrier absorption for 6 CMT samples.............................110

Table 6.3 Comparison of $\mathrm{V}_{\mathrm{O}}$ density obtained from absorption and $\mathrm{n}_{\mathrm{H}}$ from Hall measurements for 5 Airtron HYD samples.........................114

Table 7.1 Phase purity, size control, and crystal parameters for ZnO:Ga powder samples synthesized at ORNL .........................118 


\section{Chapter 1 Introduction}

\subsection{Structure and Properties of $\mathrm{ZnO}$}

Zinc oxide can be produced in three different crystal structures: wurtzite, zinc blende, and rocksalt (Figure 1.1). The hexagonal wurtzite (WZ) structure is the most stable. The cubic zincblende structure can be stable only if grown on cubic substrates. The cubic rocksalt version of $\mathrm{ZnO}$ may be obtained at relatively high pressures (Özgür, 2005).

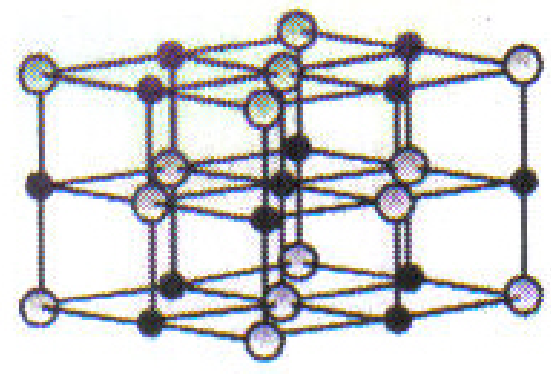

(a)

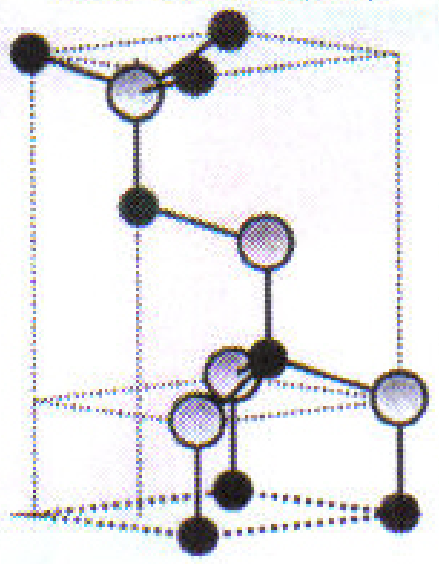

(b)

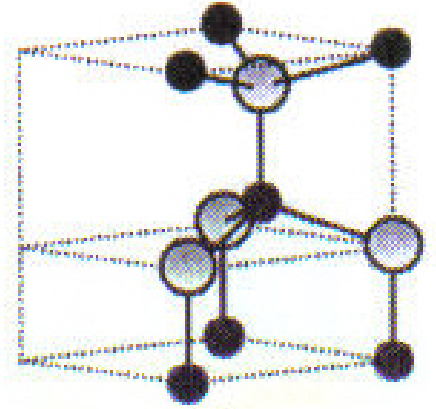

(c)

Figure 1.1. $\mathrm{ZnO}$ crystal structures: (a) rock-salt, (b) zinc blende, and (c) wurtzite. O atoms: black spheres; Zn atoms: gray spheres (from Özgür, 2005).

The WZ-structure $\mathrm{ZnO}$ consists of two interpenetrating hexagonal-close-packed (hcp) sublattices, one composed of $\mathrm{Zn}$ atoms and the other of $\mathrm{O}$ atoms, with the two basic lattice parameters $\mathrm{a}=3.252 \AA$ and $\mathrm{c}=5.213 \AA$ at room temperature. These lattice parameters are temperature dependent. The thermal-expansion coefficients, $\alpha_{\mathrm{a}}=\Delta \mathrm{a} / \mathrm{a}$ and $\alpha_{\mathrm{c}}=\Delta \mathrm{c} / \mathrm{c}$, are also temperature dependent. At $4.2 \mathrm{~K}, \mathrm{a}=3.2496 \AA$ and $\mathrm{c}=5.2042 \AA$ (Karzel, 1996). The two sublattices are displaced along the c-axis by a distance of $b$, which is the nearest neighbor distance. The ratio b/c is $0.3819 \mathrm{in} \mathrm{ZnO}$. In a $\mathrm{ZnO}$ unit cell, each $\mathrm{Zn}$ atom is surrounded by 
four $\mathrm{O}$ atoms. The $\mathrm{Zn}-\mathrm{O}$ bond length along the $\mathrm{c}$-axis is given by $\mathrm{b}=1.9875 \AA$. The other three $\mathrm{O}$ atoms in the basal plane have a $\mathrm{Zn}-\mathrm{O}$ bond length of $\mathrm{b}_{1}=1.9743 \AA$. The angle between the axial $\mathrm{Zn}-\mathrm{O}$ bond and each non-axial $\mathrm{Zn}-\mathrm{O}$ bond is $\alpha=180^{\circ}-\cos ^{-1}\left((\mathrm{c}-2 \mathrm{~b}) / \mathrm{b}_{1}\right)=$ $128.5^{\circ}$. The angle between two non-axial Zn-O bonds is $\beta=\cos -1\left(\left(b_{1}{ }^{2}-a^{2} / 2\right) / b_{1}{ }^{2}\right)=110.8^{\circ}$. $\mathrm{ZnO}$ in the $\mathrm{WZ}$ structure has a direct bandgap at room temperature (RT) with band-gap energy $\mathrm{E}_{\mathrm{g}}=3.37 \mathrm{eV}$. At $2 \mathrm{~K}$, the bandgap energy is $\mathrm{E}_{\mathrm{g}}=3.437 \mathrm{eV}$. The conduction bands are the $4 \mathrm{~s}$ states of $\mathrm{Zn}$, and the top valence bands originate from the hybrid $2 \mathrm{~s} 2 \mathrm{p}$ states of $\mathrm{O}$. Figure 1.2 shows the band structure for $\mathrm{WZ} \mathrm{ZnO} \mathrm{calculated} \mathrm{using} \mathrm{the} \mathrm{empirical}$ pseudopotential method (Bloom, 1973). Due to the spin-orbit interaction, the two top valence bands $\left(\Gamma_{5}\right.$ and $\left.\Gamma_{1}\right)$ split into three spin-degenerate bands: A $\left(\Gamma_{7}\right)$, B $\left(\Gamma_{9}\right)$, and $\mathrm{C}\left(\Gamma_{7}\right)$. The spin-orbit splitting is about $-8 \mathrm{meV}$, while the crystal-field splitting is about $42 \mathrm{meV}$.

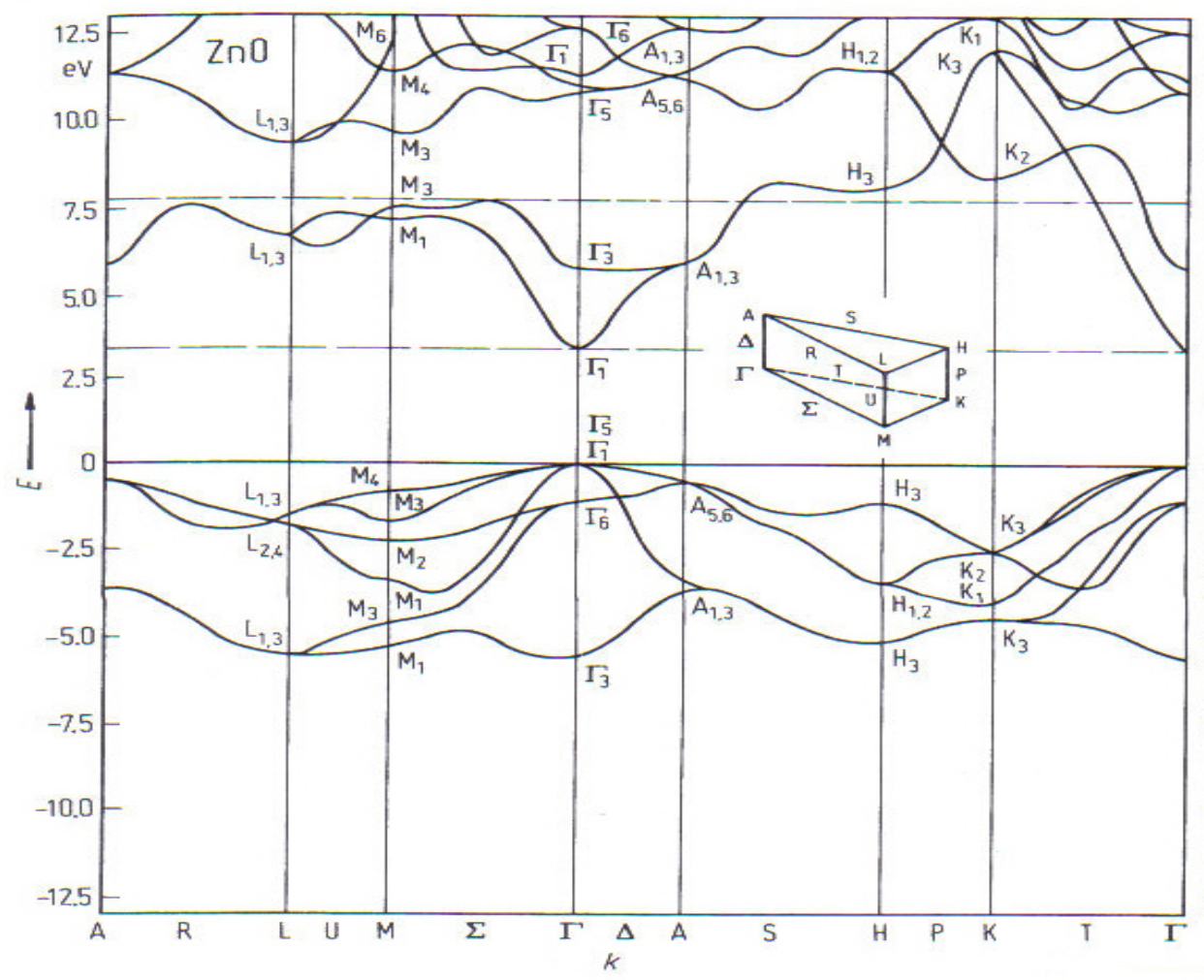

Figure 1.2. Band structure for wurtzite $\mathrm{ZnO}$ calculated using the empirical pseudo-potential method (from Bloom, 1973). 
Table 1.1: Structural and electronic parameters of $\mathrm{WZ} \mathrm{ZnO}$.

\begin{tabular}{|c|c|c|c|}
\hline Parameter & Value & Techniques & Reference \\
\hline $\begin{array}{l}\text { Lattice } \\
\text { parameters }\end{array}$ & $\begin{array}{l}\mathrm{a}=3.253 \AA(300 \mathrm{~K}) \\
\mathrm{c}=5.213 \AA(300 \mathrm{~K}) \\
\mathrm{a}=3.2496 \AA(4.2 \mathrm{~K}) \\
\mathrm{c}=5.2042 \AA(4.2 \mathrm{~K})\end{array}$ & $\begin{array}{l}\text { Capacitive method } \\
\text { X-ray diffraction }\end{array}$ & $\begin{array}{l}\text { Ibach, } 1969 \\
\text { Karzel, } 1996\end{array}$ \\
\hline $\begin{array}{l}\text { Thermal- } \\
\text { expansion } \\
\text { coefficients }\end{array}$ & $\begin{array}{c}\alpha_{\mathrm{a}}=2.9 \times 10^{-6}\left(\mathrm{~K}^{-1}\right) \\
\alpha_{\mathrm{c}}=4.75 \times 10^{-6}\left(\mathrm{~K}^{-1}\right)\end{array}$ & Capacitive method & Ibach, 1969 \\
\hline $\begin{array}{l}\text { Band-gap } \\
\text { energy }\end{array}$ & $\begin{array}{l}\mathrm{E}_{\mathrm{gA}}=3.4376 \mathrm{eV}(1.6 \mathrm{~K}) \\
\mathrm{E}_{\mathrm{gB}}=3.4405 \mathrm{eV}(1.6 \mathrm{~K}) \\
\mathrm{E}_{\mathrm{gC}}=3.4790 \mathrm{eV}(1.6 \mathrm{~K})\end{array}$ & $\begin{array}{l}\text { Exciton series limit } \\
\text { reflection }\end{array}$ & Hümmer, 1973 \\
\hline $\begin{array}{c}\text { Electron } \\
\text { effective mass }\end{array}$ & $\begin{array}{c}\mathrm{m}_{\mathrm{e}}{ }^{*}=0.24 \mathrm{~m}_{0} \text { (rigid- } \\
\text { lattice mass) } \\
\mathrm{m}_{\mathrm{e}, \mathrm{p}}{ }^{*}=0.29 \mathrm{~m}_{0} \text { ( polaron } \\
\text { mass) }\end{array}$ & Faraday rotation & Baer, 1967 \\
\hline $\begin{array}{l}\text { Hole effective } \\
\text { mass }\end{array}$ & $\begin{array}{c}\mathrm{m}_{\mathrm{h}}{ }^{*}=0.45 \mathrm{~m}_{0} \text { (rigid- } \\
\text { lattice mass) } \\
\mathrm{m}_{\mathrm{h}, \mathrm{p}}{ }^{*}=0.59 \mathrm{~m}_{0} \text { (polaron } \\
\text { mass) }\end{array}$ & $\begin{array}{c}\text { Zeeman effect } \\
1.6 \mathrm{~K} \\
\text { Magnetoreflection, } 1.6 \mathrm{~K}\end{array}$ & $\begin{array}{l}\text { Dinges, } 1970 \\
\text { Hümmer, } 1973\end{array}$ \\
\hline $\begin{array}{l}\Gamma \text {-point phonon } \\
\text { energies }\end{array}$ & $\begin{array}{l}72.8 \mathrm{meV}\left(\mathrm{E}_{1}-\mathrm{LO}\right) \\
71.2 \mathrm{meV}\left(\mathrm{A}_{1}-\mathrm{LO}\right) \\
50.7 \mathrm{meV}\left(\mathrm{E}_{1}-\mathrm{TO}\right) \\
47.1 \mathrm{meV}\left(\mathrm{A}_{1}-\mathrm{TO}\right) \\
54.3 \mathrm{meV}\left(\mathrm{E}_{2}-\mathrm{high}\right) \\
12.6 \mathrm{meV}\left(\mathrm{E}_{2}-\mathrm{low}\right)\end{array}$ & Raman spectroscopy & $\begin{array}{c}\text { Ashkenov, } 2003 \\
\text { Arguello, } 1969\end{array}$ \\
\hline $\begin{array}{l}\text { Dielectric } \\
\text { constants }\end{array}$ & $\begin{array}{l}\varepsilon(0) \perp=7.8, \varepsilon(0)_{/ /}=8.75 \\
\varepsilon(\infty) \perp=3.7, \varepsilon(\infty)_{/ /}=3.75\end{array}$ & Reflectivity & Heltemes, 1967 \\
\hline
\end{tabular}

Some important structural and electronic parameters of $\mathrm{WZ} \mathrm{ZnO}$ are listed in Table 1.1. The nearly isotropic conduction band electron effective mass is $\mathrm{m}_{\mathrm{e}}{ }^{*}=0.24 \mathrm{~m}_{0}$, where $\mathrm{m}_{0}$ is the free electron mass. The hole effective mass is $\mathrm{m}_{\mathrm{p}}{ }^{*}=0.45 \mathrm{~m}_{0}$ for the $\mathrm{A}$ and $\mathrm{B}$ valence bands. Due to the ionic nature of $\mathrm{ZnO}$, polaron effects must be considered when determining transport properties. The relation between rigid-lattice mass and polaron mass will be discussed in Chapter 2. The free exciton (FX) binding energy in $\mathrm{ZnO}$ is $60 \mathrm{meV}$. The highest $\mathrm{LO}$ phonon is the $\mathrm{E}_{1}$ mode $(72.8 \mathrm{meV})$. 


\subsection{Defects in $\mathrm{ZnO}$}

Point defects affect the electrical and optical properties of a semiconductor. Intrinsic defects such as cation and anion vacancies and interstitials can be introduced during growth or through post-growth treatments. In $\mathrm{ZnO}$, first-principle studies have addressed formation energies of several native defects (Kohan, 2000). The oxygen vacancy $\left(V_{O}\right)$ is predicted to have a lower formation energy than the zinc interstitial $\left(\mathrm{Zn}_{\mathrm{i}}\right)$. Thus, the $\mathrm{V}_{O}$ defect has been suggested to be the major native defect formed under Zn-rich conditions. In O-rich conditions, the zinc vacancy $\left(\mathrm{V}_{\mathrm{Zn}}\right)$ is believed to be the most abundant native defect. Both $\mathrm{V}_{\mathrm{O}}$ and $\mathrm{Zn}_{\mathrm{i}}$ would act as donors, while $\mathrm{V}_{\mathrm{Zn}}$ would act as acceptors.

Extrinsic defects (e.g., impurities) can act either as donors, acceptors, or isovalent defects. In $\mathrm{ZnO}$, the most common impurities believed to act as donors are hydrogen and group III elements. Hydrogen's role as a donor has been theoretically predicted (Van de Walle, 2001) but clear experimental evidence is not yet obtained. However, a proton can bond with an oxygen ion next to an acceptor defect and form an $\mathrm{OH}^{-}$complex, while the electron from the $\mathrm{H}$ atom is trapped in one of the empty bond states associated with the acceptor defect. This complex does not contribute free carriers in the conduction band and is isovalent when the acceptor is a singly ionized impurity such as Li. Thus, hydrogen can play a role in acceptor passivation even though it may not be a donor in the conventional sense.

Group III donors (Al, Ga, and In) substituting for zinc atoms are responsible for the n-type conductivity in most as-grown $\mathrm{ZnO}$ samples. These impurities have donor binding energies of about $60-70 \mathrm{meV}$. Much deeper donor states have been associated with transition metal ions (Jiang, 2007). These deeper donors do not provide high n-type conductivity, but they can play a role in passivating acceptor impurities. 
Group I elements such as $\mathrm{Li}$ and $\mathrm{Cu}$ act as accepters if they substitute for $\mathrm{Zn}$ ions in $\mathrm{ZnO}$ (Garces, 2002; Cox, 1978). Li is commonly found in crystals grown by the hydrothermal technique, but the Li acceptor energy level is deep $(\sim 0.6 \mathrm{eV})$ and is almost always totally compensated by shallow donors that are also present in the crystals. In general, a low solubility for most acceptor candidates coupled with large acceptor ionization energies make it very difficult to realize p-doping. Group $\mathrm{V}$ elements substituting for $\mathrm{O}$ ions also act as acceptors. Theoretical calculations predicted an acceptor level of $400 \mathrm{meV}$ for substitutional $\mathrm{N}_{\mathrm{O}}$, and a nearest-neighbor $\mathrm{Zn}-\mathrm{N}$ bond length of $1.88 \AA$, even shorter than the Zn-O bonds (Park, 2002). Experimental reports showed that the ionization energy of $\mathrm{N}_{\mathrm{O}}$ acceptor was about $200 \mathrm{meV}$ (Thonke, 2001; Wang, 2004). Some groups have reported success in p-type $\mathrm{ZnO}$ thin films by co-doping of group $\mathrm{V}$ acceptors such as $\mathrm{N}$ or As with group III donors (Bian, 2004; Zhuge, 2005). However, the unreasonably high hole mobility $\left(140 \sim 160 \mathrm{~cm}^{2} / \mathrm{Vs}\right)$ and poor stability and reproducibility make these results questionable.

\subsection{Synthesis of $\mathrm{ZnO}$}

Depending on the application, $\mathrm{ZnO}$ samples in single-crystal, thin-film, powder, or ceramic form may be required. In this section, a summary of several synthesis methods for single crystal and thin-film $\mathrm{ZnO}$ is given. Synthesis of powder $\mathrm{ZnO}$ samples will be discussed later in Chapter 7.

Growth of high-quality, large area, single-crystal bulk $\mathrm{ZnO}$ has been carried out mainly by three methods: seeded chemical vapor transport (SCVT), hydrothermal (HYD), and high-pressure-melt (HPM) growth. In the SCVT growth, powder or polycrystalline ZnO is heated at the hot end (about $1150{ }^{\circ} \mathrm{C}$ ) of a nearly closed horizontal tube. The sublimated 
$\mathrm{ZnO}$ reacts with a $\mathrm{H}_{2}$ carrying gas, $\mathrm{ZnO}+\mathrm{H}_{2} \rightarrow \mathrm{Zn}+\mathrm{H}_{2} \mathrm{O}$, and the mixture gas is transported to the cooler end (about $1100{ }^{\circ} \mathrm{C}$ ), where the reverse reaction takes place, and the $\mathrm{ZnO}$ product slowly deposits on a single-crystal seed (Look,1998). In the HYD growth, powder $\mathrm{ZnO}$ nutrient is mixed with a mineralizer solution of $\mathrm{Li}_{2} \mathrm{CO}_{3}, \mathrm{KOH}$, or $\mathrm{NaOH}$ in a platinum-lined autoclave, at a temperature of approximately $350{ }^{\circ} \mathrm{C}$ and a pressure between 70 and $100 \mathrm{MPa}$. Crystalline $\mathrm{ZnO}$ precipitates on single-crystal seeds suspended by Pt wires, at a rate of 10 mils per day (Suscavage, 1999). A main drawback of the HYD growth is that the product is prone to impurities due to the use of the mineralizer. The HPM growth method (Figure 1.3) employed at Cermet Inc. (Atlanta, GA) uses a pressurized induction melting apparatus where the melt is contained in a water-cooled crucible. Powder $\mathrm{ZnO}$ is used as the starting material. The heat is induced by radio frequency energy until the $\mathrm{ZnO}$ is molten at about $1900{ }^{\circ} \mathrm{C}$. Then the crucible is slowly lowered away from the heated zone to allow crystallization (Nause, 1999).

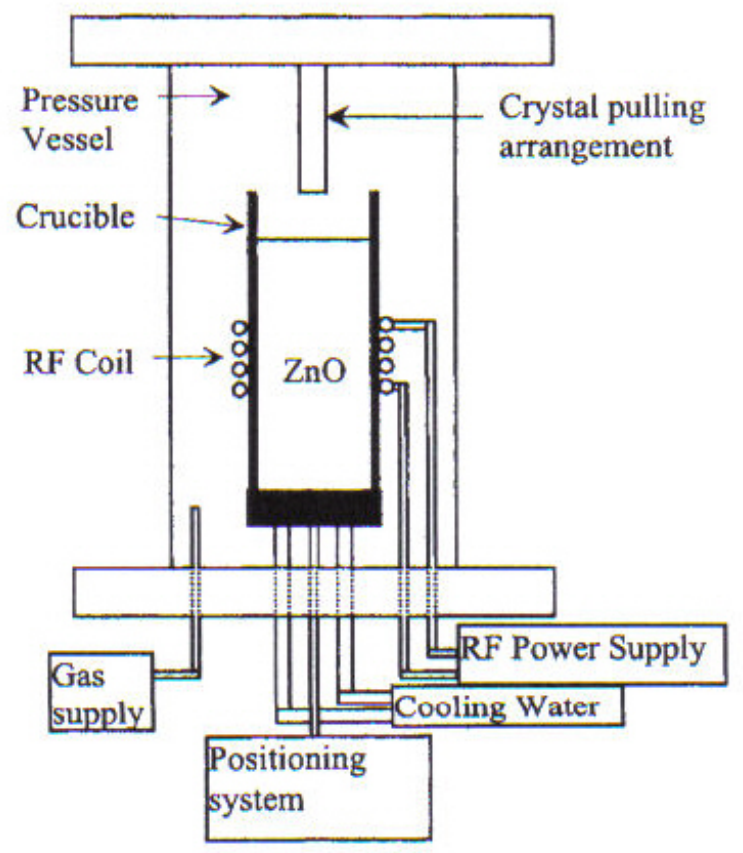

Figure 1.3. The high-pressure-melt (HPM) growth apparatus at Cermet Inc. (from Nause, 1999). 
Early thin-film growth studies of $\mathrm{ZnO}$ used techniques such as magnetron sputtering and chemical vapor deposition on sapphire or diamond substrates (Hachigo,1994; Gardeniers, 1998). The films were mainly polycrystalline. Lately, by using growth techniques such as molecular-beam epitaxy (MBE), pulsed-laser deposition (PLD), and metal-organic chemical vapor deposition (MOCVD), which allow a finer control over the deposition procedure, highquality single-crystal films have been prepared (Chen, 1998; Vispute, 1998; Liu, 2000).

One of the problems that $\mathrm{ZnO}$ thin-film growers face is the choice of substrates. Large lattice parameter mismatches cause strains and dislocations in epitaxial films. Sapphire $\left(\alpha-\mathrm{Al}_{2} \mathrm{O}_{3}\right)$ substrates are commonly used in $\mathrm{ZnO}$ growth, although the lattice mismatch along the a-axis with $\mathrm{ZnO}$ is large (about 18.4\%). Hexagonal structure AlN and $6 \mathrm{H}-\mathrm{SiC}$ are better choices since they give a-axis lattice mismatches of about $4.5 \%$ and $3.5 \%$, respectively. The parameters of $\mathrm{GaN}$ are $\mathrm{a}=3.189 \AA$ and $\mathrm{c}=5.185 \AA$, and the mismatch to $\mathrm{ZnO}$ is less than $2 \%$ in both a- and c-axes. However, bulk GaN crystals are scarce. Hexagonal $\mathrm{ScAlMgO}_{4}$ substrates were used to grow $\mathrm{ZnO}$ by laser-assisted $\mathrm{MBE}$ (Ohtomo, 1999). $\mathrm{ScAlMgO}_{4}$ has lattice parameters $\mathrm{a}=3.246 \AA$ and $\mathrm{c}=25.195 \AA$, thus giving an a-axis mismatch as small as $0.09 \%$.

\subsection{ZnO Applications: Scintillators and More}

The direct wide-band-gap energy, large FX binding energy (60 meV), and moderate density $\left(5.67 \mathrm{~g} / \mathrm{cm}^{3}\right)$ make $\mathrm{ZnO}$ suitable for applications as an ultraviolet scintillator. A scintillator material absorbs ionizing radiation and converts the incident energy into light (called scintillation). The light is usually in the visible or ultraviolet spectral ranges and can then be detected using a photomultiplier tube (PMT). The first scintillator materials included 
$\mathrm{CaWO}_{4}$, used by Pupin (Bushong, 1975) as X-ray screen within a few months of the discovery of X-rays by Conrad Röentgen; uranyl, used by Becquerel to discover radioactivity; and $\mathrm{ZnS}$, used by Crookes in his spinthariscope to count alpha-particles from radium (Crookes, 1903), and by Rutherford in the discovery of the atomic nucleus (Rutherford, 1930). Since the development of the PMT and its coupled use with thallium-activated NaI (NaI:Tl) in the 1940s, strong scintillation from more than a dozen inorganic materials were demonstrated over the following two decades (Hofstadter, 1949; Gwin, 1963). In 1966, W. Lehmann found that the scintillation decay time of $\mathrm{ZnO}: \mathrm{Ga}$ and $\mathrm{CdS}$ :In powders were less than a nanosecond (Lehmann, 1966). During the last two decades, research and development of scintillator materials saw another renaissance prompted by the need of scintillation for precision calorimetry in high energy physics, medical imaging (e.g., in positron emission tomography, or PET), space physics, geophysical exploration and other scientific and industrial applications (Rodnyi, 1997; Derenzo, 2003).

Reviews of the scintillation process can be found in the literature (Rodnyi, 1997; Derenzo, 2003). The scintillation process can be divided into a sequence of stages.

1. The absorption of the incident radiation and subsequent creation of energetic primary electrons (hot electrons) and inner shell holes. This happens in a time scale of about $10^{-15}-10^{-13} \mathrm{sec}$.

2. Relaxation of the hot electrons and inner shell holes through intraband transitions and electron-phonon interactions. This takes about $10^{-12}-10^{-11} \mathrm{sec}$, and results in lower energy (secondary) electrons and holes.

3. Thermalization of the secondary electrons and holes and formation of electronhole pairs with energies on the order of $E_{g}$. Energy is then transferred to the luminescence 
centers leaving them in excited states. Depending on the type of luminescence centers, this can take as short as $10^{-12} \mathrm{~s}$, or as long as $10^{-8} \mathrm{~s}$, which gives the intrinsic rise time of scintillation light.

4. The emission of scintillation light from the luminescent centers. The fast radiative process can be as short as $10^{-9} \mathrm{~s}$ for recombinations involving free electrons and holes, free and bound excitons, and core-valence recombination. Slower radiative processes can take seconds or minutes for forbidden transitions. These types of scintillation processes are not appropriate for coincidence detectors.

An ideal scintillator should have high energy-conversion efficiency (also called light yield), short rise and decay times for better time resolving measurements, chemical and mechanical stability over wide range of extrinsic conditions such as temperature and time, radiation hardness, etc. As mentioned above, $\mathrm{ZnO}$ :Ga demonstrated sub-nanosecond photoluminescence, cathodoluminescence, and X-ray excited luminescence (Lehmann, 1966; Derenzo, 2002, Lorenz, 2006). Also, the free exciton in $\mathrm{ZnO}$ :In has a spontaneous radiative lifetime of 290 ps at $85 \mathrm{~K}$ and 440 ps at $296 \mathrm{~K}$, shorter than most of the known inorganic scintillators, while the bound exciton has a lifetime as short as 50 ps from $85 \mathrm{~K}$ down to $16 \mathrm{~K}$ (Wilkinson, 2005). This makes donor-doped $\mathrm{ZnO}$ a suitable candidate for scintillator applications requiring super-fast response and high energy resolution. On the other hand, the medium density $\left(5.67 \mathrm{~g} / \mathrm{cm}^{3}\right)$ of $\mathrm{ZnO}$ makes it more appealing than plastic scintillators having similar light yield and decay times, since the larger density of $\mathrm{ZnO}$ leads to higher stopping power and hence less material needed for a detector (Simpson, 2003). On the other hand, the relatively low light yield has been a limit to the widespread use of $\mathrm{ZnO}$ as a scintillator, although it shows the highest luminosity compared to other sub-nanosecond 
scintillators $\mathrm{CuI}, \mathrm{HgI}_{2}, \mathrm{PbI}_{2}$, and $\mathrm{CdS}: \mathrm{In}$ (Derenzo, 2002). The intrinsic reason for the low light yield has to do with the large value of the bandgap energy (Rodnyi, 1997). The scintillation light yield $\mathrm{L}_{\mathrm{R}}$ is the number of emitted photons per unit of absorbed radiation energy $\mathrm{E}_{\gamma}$ (usually $1 \mathrm{MeV}$ ) and is expressed as

$$
L_{R}=\frac{N_{p h}}{E_{\gamma}}=\frac{\alpha}{\beta E_{g}}
$$

where $\alpha$ is the average number of photons produced by an electron-hole pair $(\alpha \leq 1)$ and $\beta$ is the ratio of $\xi_{\text {eh }}$, the average energy needed to create an e-h pair, to the band gap energy $E_{g}$. The value of $\beta=\xi_{\text {eh }} / E_{\mathrm{g}}$ is about 1.5 to 2 for ionic crystals.

Another reason for the low light yield in $\mathrm{ZnO}$ is due to the strong self-absorption of the near-band-edge (NBE) emission. The effect of self-absorption on the detected line shape of light emission from semiconductors was reviewed by J. I. Pankove (Pankove, 1971). If a spectrum $L_{0}(v)$ is emitted at a point in the sample at a distance $d$ from the exiting surface, the radiated spectrum from the sample surface is given by

$$
\mathrm{L}(v)=(1-\mathrm{R}) \mathrm{L}_{0}(v) \mathrm{e}^{-\alpha(v) d}
$$

where $\mathrm{R}$ is the reflectance at the surface and $\alpha(v)$ is the frequency-dependent absorption coefficient. More rigorously, if the recombination occurs uniformly in the specimen of thickness $t$, the external spectrum will be given by

$$
L(v)=(1-R) \frac{l_{0}(v)}{t} \int_{o}^{t} e^{-\alpha(v) x} d x=(1-R) L_{0}(v) \frac{1-e^{-\alpha(\theta) t}}{\alpha t}
$$

As shown in Figure 1.4 (included in Pankove, 1971), a luminescence spectrum from a very thin (thickness $=13 \mu$ ) germanium sample, the observed high-energy (shorter wavelength) peak associated with the direct transition is much weaker than that associated with the indirect transition at longer wavelength (Ge has an indirect band gap). However, 
after correction for the self-absorption effect, the direct emission peak is now more intense than the indirect peak. This result, more closely resembling the emission occurring within the sample, occurs because of the higher probability of direct recombination. The correction also helped relocate the peak position of the direct emission (originally from Haynes, 1955).

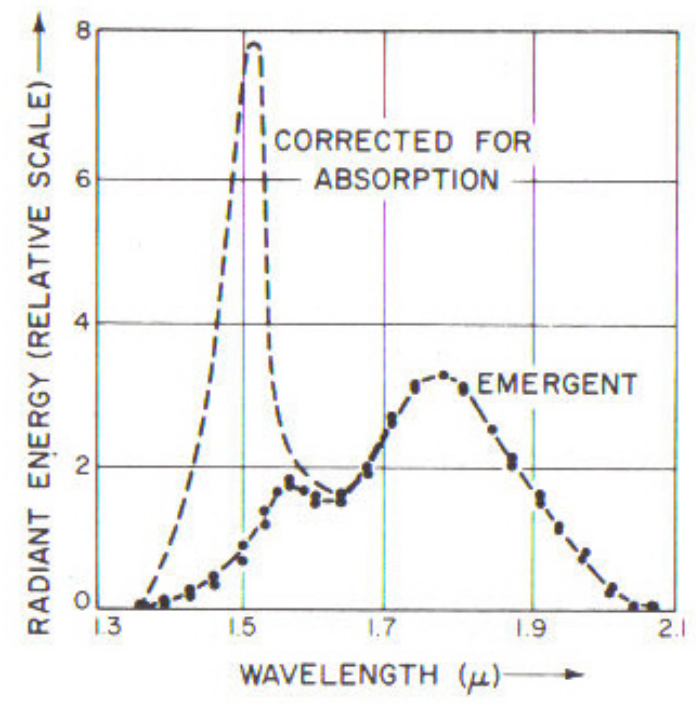

Figure 1.4. Illustrative example of luminescence measured from a $13-\mu \mathrm{m}$-thick germanium sample, and effect of correcting for sample self-absorption. The corrected data (dashed line) has much more intense direct transition peak, and the peak energy is shifted to shorter wavelengths (figure from Haynes, 1955).

Self-absorption of NBE in $\mathrm{ZnO}$ was pointed out for single crystals from Cermet which were studied by Simpson et al. (Simpson, 2003; Lorenz, 2006). To reduce the selfabsorption effect, the emission peak should be displaced further below the band gap. In fact, if the main NBE is not free exciton (FX), but some other recombination such as the phononassisted free exciton emission (FX-LO), the self-absorption effect can also be less of a concern. $\mathrm{ZnO}$ single crystals grown from the SCVT method have demonstrated bright UV emission with peak around $3.26 \mathrm{eV}$ at RT, which is mainly due to the FX-1LO emission (Wang, 2003). W. Lehmann's ZnO:Ga powder sample has a UV peak at about $3.22 \mathrm{eV}$ (Lehmann, 1966). In these samples, the NBE emission has a better chance surviving the selfabsorption effect. 
Besides being a good scintillator, powder and polycrystalline $\mathrm{ZnO}$ have been developed for use in cosmetics such as sunscreen creams, and as a catalyst in some chemical reactions. The recent availability of high-quality, large-area single crystal bulk and epitaxial $\mathrm{ZnO}$ has led to a resurgence of study on this wide-band-gap material. Some other applications include $\mathrm{ZnO-based} \mathrm{ultraviolet} \mathrm{photo-detectors,} \mathrm{light-emitting} \mathrm{diodes} \mathrm{(LED),}$ transparent thin-film transistors (TTFT), solar cells, and gas sensors. One of the most efficient solid-state light emitters are $\mathrm{p}$-n junctions. Since p-type $\mathrm{ZnO}$ is difficult to make, and a $\mathrm{ZnO}$ homo-junction is not yet realistic, the best $\mathrm{ZnO}$-based emitter to date is a heterojunction $\mathrm{n}-\mathrm{ZnO} / \mathrm{p}-\mathrm{AlGaN} / \mathrm{n}-\mathrm{SiC}$, which produces intense $390 \pm 1 \mathrm{~nm}$ emission at both $300 \mathrm{~K}$ and $500 \mathrm{~K}$ (Look, 2004). Due to its wide band-gap, $\mathrm{ZnO}$ is transparent and will not degrade on exposure to visible light, which is the basic requirement for a good TTFT material. The high voltage, temperature and radiation tolerance, and availability of high-quality thin films at relatively low temperatures on a variety of substrates lead to success in ZnO-based TTFT fabrication. ZnO TTFT fabricated by rf magnetron sputtering on heavily doped $\mathrm{n}-\mathrm{Si}$ substrates exhibited "best" on-off ratios $>10^{6}$, with optical transparencies $>80 \%$ for wavelengths $>400 \mathrm{~nm}$ (Carcia, 2003).

A crucial step in optoelectronic device design is the realization of a tunable band-gap, or band-gap engineering. The wide-band gap $\mathrm{ZnO}\left(\mathrm{E}_{\mathrm{g}}=3.37 \mathrm{eV}\right)$ can be alloyed with other Group II elements to form a ternary alloy $\mathrm{A}_{\mathrm{x}} \mathrm{Zn}_{1-\mathrm{x}} \mathrm{O}$. The most common choices for $\mathrm{A}$ are Mg or Cd. The band-gap energy for the ternary alloy can be described by a quadratic relation in $\mathrm{x}$, the molar composition of element A (Van Vechten, 1970)

$$
\mathrm{E}_{\mathrm{g}}(\mathrm{x})=(1-\mathrm{x}) \mathrm{E}_{\mathrm{g}}^{\mathrm{ZnO}}+\mathrm{xE}_{\mathrm{g}}{ }^{\mathrm{AO}}-\mathrm{bx}(1-\mathrm{x})
$$


where $\mathrm{b}$ is the bowing parameter. The bandgap energy $\mathrm{E}_{\mathrm{g}}{ }^{\mathrm{AO}}$ is $7.7 \mathrm{eV}$ for $\mathrm{MgO}$, and $2.3 \mathrm{eV}$ for $\mathrm{CdO}$, both of cubic rocksalt structure. Synthesis of $\mathrm{Mg}_{\mathrm{x}} \mathrm{Zn}_{1-\mathrm{x}} \mathrm{O}(\mathrm{MZO})$ has been reported with $\mathrm{x}$ up to 0.33 and tuning of $\mathrm{E}_{\mathrm{g}}$ up to $3.9 \mathrm{eV}$ (Ohtomo, 1998). A decrease in $\mathrm{E}_{\mathrm{g}}$ to blue visible wavelengths is possible using $\mathrm{Cd}_{\mathrm{x}} \mathrm{Zn}_{1-\mathrm{x}} \mathrm{O}(\mathrm{CZO})$, and success was reported for deposition of CZO thin films $(0 \leq \mathrm{x} \leq 0.53)$ on glass and sapphire substrates using dc reactive magnetron sputtering method, with $\mathrm{E}_{\mathrm{g}}$ being tuned down to $2.65 \mathrm{eV}$ (Ma, 2004).

\subsection{Motivation and Goals}

Part of my work is related to the development of $\mathrm{ZnO}$-based scintillators as the coating material of an alpha $(\alpha)$-particle detector in a deuterium-tritium (D-T) neutron generator at Oak Ridge National Laboratory (ORNL) (see Figure 1.5). A fast, highefficiency associated $\alpha$-particle detector allows precise determination of the direction and time of origin of the electronically collimated neutron beam with an energy of $14.1 \mathrm{MeV}$ (Neal, 2008). Integration of an associated $\alpha$-particle detector with a sealed-tube D-T generator presents such challenges as a high-temperature bake-out $\left(350{ }^{\circ} \mathrm{C}\right.$ for $>72$ hours $)$,

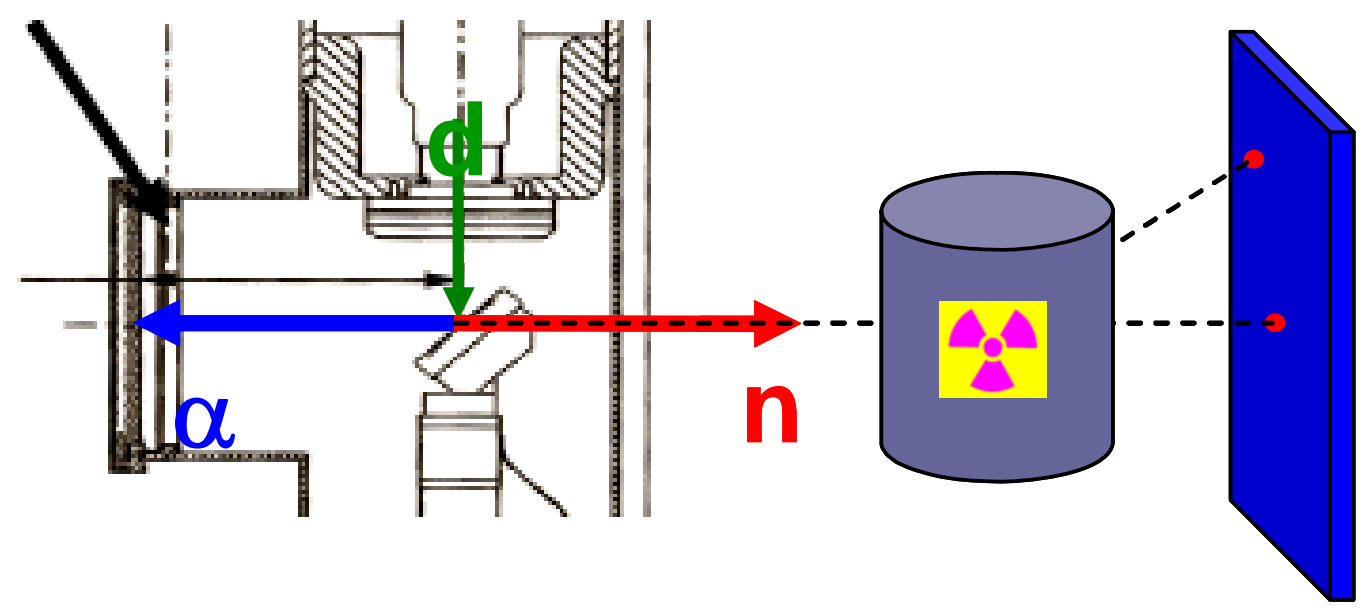

Figure 1.5 A schematic of the deuterium-tritium (D-T) neutron generator and associated alpha-particle detector (ORNL). The black arrow (left, upper corner) indicates the position of the $\mathrm{ZnO}: \mathrm{Ga}$ scintillation screen on the alpha-particle detector (from Neal, 2007). 
the exclusion of organics, and a harsh radiation environment. The sub-nanosecond response of NBE emission plus the robust thermal and mechanical properties makes $\mathrm{ZnO}$ :Ga the ideal candidate for this purpose. Seeking the brightest $\mathrm{ZnO}$ :Ga emitter from different synthesis methods such as single crystals, powder, and ceramics, and determination of optimum postgrowth treatments to enhance the fast ultraviolet NBE emissions is a focus of my study. My work also aims to understand the origin of the undesired slow visible emissions and help seek ways to reduce or eliminate them. These goals form the ultimate objective of the ORNL project and the motivation for this work.

My specific goals in this study include:

1. Understanding the recombination mechanism of NBE emission from different single crystal, powder, and ceramic ZnO samples. Room temperature (RT) PL line-shape analysis, liquid helium temperature and temperature-dependent PL experiments are combined for distinguishing and modeling the main transition in each sample.

2. Understanding the energy levels and concentrations of defects in single crystal $\mathrm{ZnO}$ samples through temperature dependent Hall measurements and analysis. To get meaningful comparison between different samples, an intrinsic mobility limit has to be first setup by carefully selecting the critical material parameters involved in the calculation of mobilities related to intrinsic scatterings.

3. Understanding the identity of shallow and deep level defects in $\mathrm{ZnO}$ samples of different forms and from different growth methods, through a correlated study of PL, x-ray excited luminescence, Hall measurements, Fourier transform infrared (FTIR) and electron paramagnetic resonance (EPR) data. Evaluation of the effect of different after-growth treatments on the edge emission, visible emission, and absorption will also be made. 


\section{Chapter 2 Photoluminescence and Hall Effect}

\subsection{Photoluminescence}

The general term luminescence is used when referring to the spontaneous recombination of an electron-hole pair from an excited state to ground state by releasing the excess energy in the form of a photon emission. The excited state is reached by absorption of external energy introduced by such as photons, X-rays, electrons or electric currents, and the resulting luminescence is named as photoluminescence (PL), radioluminescence, cathodoluminescence, and electroluminescence, respectively. Internal energy can also induce luminescence, such as thermoluminescence and triboluminescence (strain-induced luminescence) (Pankove, 1971).

In the photoluminescence (PL) process, a photon is absorbed by the semiconductor (i.e., optical excitation) and creates an electron-hole pair, which then recombines and emits another photon. Between the excitation and emission, non-radiative energy dissipation processes can take place before the excited electron-hole pair reaches the lowest available state. The recombination of the e-h pairs from different states gives rise to different luminescence bands, as will be discussed below.

\subsubsection{Free Excitons}

In a relatively pure sample, an excited electron in the conduction band $(\mathrm{CB})$ and a free hole left in the valence band (VB) attract each other via the Coulomb interaction, forming neutral bound pairs similar to a hydrogen atom. These pairs can have an effective radius of several lattice constants, or can be tightly confined to the site of excitation. The 
former is called a free exciton (FX), or Wannier-Mott exciton, which is often observed in most semiconductors. The latter form is called a Frenkel exciton, which can be found in insulators or molecular crystals.

In a free exciton of the Wannier-Mott variety, the binding is weak and the distance between the electron and the hole is large. The free exciton can be described as moving in a uniform dielectric material with a relative dielectric constant $\varepsilon_{\mathrm{r}}$. Applying the Bohr model to calculate the energy of the nth level below the ionization limit (i.e., a free electron in the $\mathrm{CB}$ and a free hole in the VB), one gets

$$
E(n)=-\frac{\mu}{m_{0}} \frac{1}{\varepsilon_{r}^{2}} \frac{R_{H}}{n^{2}}=-\frac{R_{X}}{n^{2}}
$$

where $\mu$ is the reduced effective mass of the exciton,

$$
\frac{1}{\mu}=\frac{1}{m_{e}^{*}}+\frac{1}{m_{h}^{*}}
$$

In Eq. 2.1, $\mathrm{R}_{\mathrm{H}}=13.6 \mathrm{eV}$ is the Rydberg constant of the hydrogen atom. The exciton Rydberg constant $R_{x}$, or binding energy, is given by $R_{x}=\left(\mu / m_{0} \varepsilon_{r}^{2}\right) R_{H}$. The energy of the ground state $(n=1)$ of a free exciton is thus $E_{x}=E_{g}-R_{x}$. The effective Bohr radius of the ground state of a free exciton is

$$
a_{X}=\frac{m_{0}}{\mu} \varepsilon_{r} a_{H}
$$

where $\mathrm{a}_{\mathrm{H}}=0.529 \AA$ is the Bohr radius of the hydrogen atom. When the FX density is large and the separation between two excitons is close to the exciton diameter, the exciton wave functions overlap and discrete bound excitons no longer form. The FX density at which the separation between two excitons becomes $2 \mathrm{a}_{\mathrm{X}}$ is called the Mott density $\mathrm{N}_{\text {Mott, }}$

$$
N_{\text {Mott }}=\frac{3}{4 \pi a_{X}^{3}}
$$


In $\mathrm{ZnO}$, the $\mathrm{FX}$ binding energy $\mathrm{R}_{\mathrm{X}}$ is about $60 \mathrm{meV}$ and the effective Bohr radius is $\mathrm{a}_{\mathrm{X}} \approx 20$ $\AA$, thus the Mott density is $\mathrm{N}_{\text {Mott }} \approx 3 \times 10^{19} \mathrm{~cm}^{-3}$.

During a FX recombination, the emitted photon energy is given by

$$
\mathrm{h} v=\mathrm{E}_{\mathrm{x}}(\mathrm{n})=\mathrm{E}_{\mathrm{g}}-\mathrm{R}_{\mathrm{X}} / \mathrm{n}^{2}
$$

By comparing the observed energies of the ground $(n=1)$ and first excited state $(n=2)$ lines, the FX binding energy $R_{X}$ can be determined:

$$
\mathrm{R}_{\mathrm{X}}=(4 / 3)[\mathrm{h} v(\mathrm{n}=2)-\mathrm{h} v(\mathrm{n}=1)]
$$

However, this is not easily observed in most semiconductors, since the intensities of higher order peaks decrease as $\sim \mathrm{n}^{-3}$ (Elliott, 1957).

As given in Table 1.1, the rigid-lattice effective electron mass in $\mathrm{ZnO}$ is $0.24 \mathrm{~m}_{0}$, and that of the hole is $0.45 \mathrm{~m}_{0}$, where $\mathrm{m}_{0}$ is the free electron mass. This gives a reduced mass of the exciton as $\mu=0.16 \mathrm{~m}_{0}$. To get the FX binding energy $R_{x}=60 \mathrm{meV}$, one needs a relative dielectric constant $\varepsilon_{\mathrm{r}}=6.0$. This value falls between the values of the static and highfrequency dielectric constants, and may be ascribed to the screening effect due to the overlapping of electron and hole wave functions. The static dielectric constant $\left(\varepsilon_{\mathrm{s}}\right)$ can be used for $\varepsilon_{\mathrm{r}}$ in Equations 2.1 and 2.3, as long as the exciton binding energy $\left(\mathrm{R}_{\mathrm{x}}\right)$ is small compared to the optical phonon energy $\left(\hbar \omega_{\mathrm{LO}}\right)$. However, in materials such as $\mathrm{ZnO}$, these two values are close, as given in Table 1.1. In this situation, the Haken potential approach can be used to find the appropriate value of $\varepsilon_{\mathrm{r}}$, which is a function of the separation of electrons and holes in the material (Klingshirn, 1997):

$$
\frac{1}{\varepsilon_{r}\left(r_{e, h}\right)}=\frac{1}{\varepsilon_{b}}-\left(\frac{1}{\varepsilon_{b}}-\frac{1}{\varepsilon_{s}}\right)\left[1-\frac{\exp \left(-r_{e, h} / r_{e}^{p}\right)-\exp \left(-r_{e, h} / r_{h}^{p}\right)}{2}\right]
$$


Here $r_{e, h}$ is the electron-hole separation, $\varepsilon_{\mathrm{b}}$ and $\varepsilon_{\mathrm{s}}$ are high-frequency and static dielectric constants, and $r_{e}^{p}$ and $r_{h}^{p}$ are the polaron radii of electron and hole, respectively.

In a compound semiconductor, the lattice formed by the cation and anion ions will be perturbed by the excess carrier, either an electron or a hole. When an electron carrier moves within the lattice, the attraction between the electron and the nearby cations and the repulsion between the electron and nearby anions cause a displacement of the lattice and form a phonon cloud called a polaron that moves along with the electron. This polaron cloud can have an effective radius several times larger than the lattice parameters, and it is heavier than a free electron in a "rigid lattice". A polaron can also be formed around a hole. The effective mass of a polaron is given by (Fox, 2001):

$$
m_{e, h}^{p}=m_{e, h}^{r . l .}\left(\frac{1}{1-\alpha_{e, h} / 6}\right) \approx m_{e, h}^{r \cdot l \cdot}\left(1+\alpha_{e, h} / 6\right)
$$

Here $m_{e, h}^{p}$ is the effective polaron mass for electron or hole, and $m_{e, h}^{r . l}$ is the effective rigidlattice mass for electron or hole. The dimensionless constant $\alpha_{e, h}$ is called the Fröhlich electron-phonon coupling constant, and is given by

$$
\alpha_{e, h}=\frac{e^{2}}{8 \pi \varepsilon_{0} \hbar \omega_{L O}}\left(\frac{2 m_{e, h}^{r l l} \omega_{L O}}{\hbar}\right)^{1 / 2}\left(\frac{1}{\varepsilon_{b}}-\frac{1}{\varepsilon_{s}}\right)
$$

And the radius of the phonon, $r_{e, h}^{p}$, is given by

$$
r_{e, h}^{p}=\left(\frac{\hbar}{2 m_{e, h}^{r . l} \omega_{L O}}\right)^{1 / 2}
$$

For $\mathrm{ZnO}$, using Eq. 2.10 and parameters given in Table 1.1, i.e., $m_{e, h}^{r . l .}=0.24 \mathrm{~m}_{0}$ (for electron) and $0.45 \mathrm{~m}_{0}$ (for hole), and $\hbar \omega_{\mathrm{LO}}=0.072 \mathrm{meV}$, the effective polaron radii are found to be $r_{e}^{p}=15 \AA$, and $r_{h}^{p}=11 \AA$, respectively. Using the free exciton radius $\mathrm{ax}_{\mathrm{X}}=20 \AA$ to 
represent the electron-hole separation distance, the effective dielectric constant for $\mathrm{ZnO}$ is found to be $\varepsilon_{\mathrm{r}}=6.3$ from Eq. 2.7.

The FX transition can also occur with the emission of one or more optical phonons. In this case, the energy of the emission is an amount $\mathrm{m \hbar} \omega$ lower than the FX energy, where $m$ is the number of phonons emitted per transition and $\hbar \omega$ is the phonon energy. The average number of phonons involved in the radiative recombination is often referred to as the HuangRhys factor. The transition probability of the phonon-assisted emissions is small compared to a zero-phonon direct FX transition, so the Huang-Rhys factor is expected to be $<1$. However, if the optical phonon energy is large, e.g., $\hbar \omega=72 \mathrm{meV}$ in $\mathrm{ZnO}$, the FX-1LO and FX-2LO photons have a greater chance to escape the sample because their energies are far below the energy gap and occur where the material is more transparent.

The FX-LO transitions have different PL line shapes than that for the zero-phonon FX line. The FX line has a symmetric Gaussian line shape given by (Bebb, 1972)

$$
F(E) \sim \exp \left[-\frac{\left(E-E_{x}\right)^{2}}{2 \sigma^{2}}\right] \exp \left(-\frac{E-E_{x}}{k_{B} T}\right)
$$

where $\mathrm{E}$ is the energy of the emitted photon, and $\mathrm{E}_{\mathrm{x}}$ is the $\mathrm{FX}$ transition energy. At a given temperature $T$, the peak energy of the FX line is $E_{m}=E_{x}-\sigma^{2} / k_{B} T$. The quantity $\sigma$ in Eq. 2.11 is proportional to the full-width at half-maximum (FWHM) of the FX peak as $\sigma=$ $\mathrm{FWHM} /(8 \ln 2)^{1 / 2} . \approx(0.42) \mathrm{FWHM}$. If the FWHM varies with temperature as $\mathrm{FWHM} \sim \mathrm{k}_{\mathrm{B}} \mathrm{T}$, the displacement of the FX peak from $E_{x}$ to lower energy is $\Delta E=\sigma^{2} / k_{B} T=\left[\left(k_{B} T\right)^{2} /(8 \ln 2)\right]$ $/ \mathrm{k}_{\mathrm{B}} \mathrm{T} \approx \mathrm{k}_{\mathrm{B}} \mathrm{T} /(8 \ln 2) \approx 5 \mathrm{meV}$ at $300 \mathrm{~K}$. More rigorous study found that the value of $\sigma$ could vary as a function of temperature given by $\sigma^{2}=\sigma_{0}{ }^{2}+\lambda^{2} \operatorname{coth}\left(\hbar \omega / 2 \mathrm{k}_{\mathrm{B}} T\right)$, where $\hbar \omega$ is the emitted phonon energy and $\lambda$ is a constant (Klick, 1957). 
The first phonon-assisted line (FX-1LO) has an asymmetric line shape given by (Bebb, 1972)

$$
F(E) \sim \varepsilon^{1 / 2}\left[\exp \left(-\varepsilon / \mathrm{k}_{\mathrm{B}} \mathrm{T}\right)\right] \mathrm{W}(\varepsilon)
$$

where $\varepsilon=\mathrm{E}-\mathrm{E}_{\mathrm{x}}+\hbar \omega_{\mathrm{LO}}$ is the kinetic energy of the exciton, and $\mathrm{W}(\varepsilon) \sim \varepsilon^{\mathrm{p}}$ is the transition probability rate function with $0<\mathrm{p}<1$ for FX-1LO. At low temperatures, the FX and its phonon replica lines are well resolved. As temperature increases, line broadening and overlapping of emission bands occurs. Line-shape analysis on PL spectra from single crystal $\mathrm{ZnO}$ samples will be discussed in more detail in Chapter 4.

\subsubsection{Bound Excitons}

In the presence of point defects, an exciton can be bound to a neutral or charged defect and become localized. An exciton bound to a donor (DBX) or an acceptor (ABX) has an energy lower than that of the FX by a given amount. This amount of energy is called the binding energy $\left(\mathrm{E}_{\mathrm{B}}\right)$ of the bound exciton. $\mathrm{E}_{\mathrm{B}}$ is proportional to the corresponding donor or acceptor ionization energy $\mathrm{E}_{\mathrm{D}}$ or $\mathrm{E}_{\mathrm{A}}$ (Haynes, 1960).

One characteristic of a bound exciton is that the emission peak is very narrow, with typical line width of $0.1 \mathrm{meV}$, compared to about $1 \mathrm{meV}$ for FX at low temperature. Another characteristic of $\mathrm{BX}$ emission is its fast thermal quenching. As temperature increases, the $\mathrm{BX}$ first dissociates into a FX and later on the FX will dissociate and leave a free electron in the $\mathrm{CB}$ and a free hole in the VB. As a donor bound exciton (DBX) transition occurs, the donor left behind can be either in its ground state or in one of its excited states. When the second case happens, the emitted photon energy will be smaller than the DBX energy. This emission is called the two-electron-satellite (TES) line of the DBX emission. The energy 
difference between a DBX and its TES line is the energy needed to put the donor in its excited state. Measurement of this energy difference can be used to determine the donor ionization energy $E_{D}$. A similar process can happen in the $\mathrm{ABX}$ transition, where the transition leaves the acceptor in its excited state and emits a two-hole-satellite (THS) line with respect to the $\mathrm{ABX}$ line. Measuring the energy difference between an $\mathrm{ABX}$ line and its THS line helps determine the acceptor ionization energy $\mathrm{E}_{\mathrm{A}}$. The use of TES lines to determine donor energies in $\mathrm{ZnO}$ has been reported (Meyer, 2004).

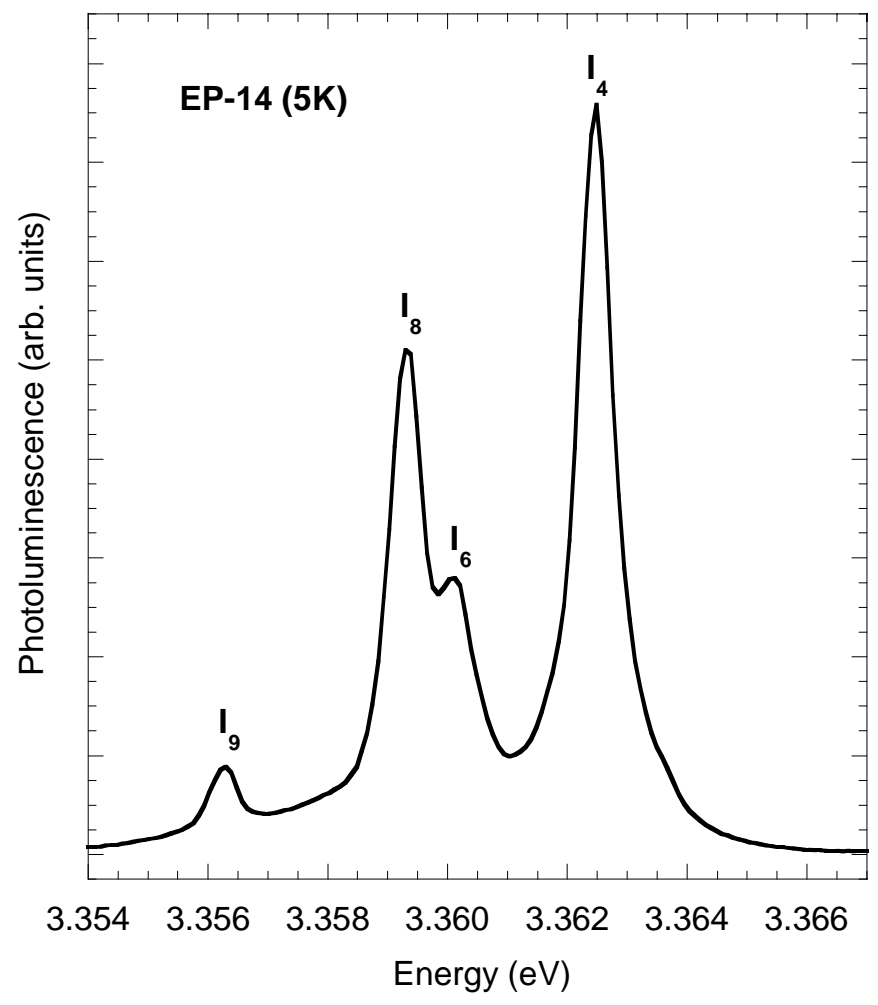

Figure 2.1 Four DBX PL lines at 5 $\mathrm{K}$ from a single-crystal $\mathrm{ZnO}$ grown by the SCVT method at Eagle-Picher Inc. (Miami, OK). The labels are described in the text.

Figure 2.1 shows four discrete donor-bound-exciton (DBX) peaks observed at $5 \mathrm{~K}$ from a single-crystal $\mathrm{ZnO}$ sample prepared by the seeded chemical-vapor-transport (SCVT) method at Eagle-Picher inc. (Miami, OK). The strongest DBX line $\left(\mathrm{I}_{4}\right)$ in this sample is related to hydrogen, while the others are $\mathrm{DBX}$ lines bound to group III donors $\mathrm{Al}\left(\mathrm{I}_{6}\right), \mathrm{Ga}\left(\mathrm{I}_{8}\right)$, and In ( $\left.\mathrm{I}_{9}\right)$ (Meyer, 2004). 


\subsubsection{Band-to-band transitions}

A free electron in the conduction band can recombine with a free hole in the valence band without forming an exciton. The recombination can be a direct transition, where the electron and hole states have the same k-value, or can be indirect transition, where momentum conservation is satisfied with the help of phonons. In both cases, the resulting photon energy can be higher than the band-gap energy. When the Boltzmann distribution can be used in description of the density of states in the conduction band, the direct band-toband transition will have a line shape of

$$
\mathrm{I}(\mathrm{h} v) \sim\left(\mathrm{h} v-\mathrm{E}_{\mathrm{g}}\right)^{1 / 2} \exp \left[-\left(\mathrm{h} v-\mathrm{E}_{\mathrm{g}}\right) / \mathrm{k}_{\mathrm{B}} \mathrm{T}\right]
$$

The emission will have an abrupt low-energy cut-off at $h v=E_{g}$ (i.e., this line shape does not include an Urbach tail). On the higher energy side, the emission decreases exponentially. The maximum intensity occurs at an energy given by $\mathrm{E}_{\mathrm{g}}+(1 / 2) \mathrm{k}_{\mathrm{B}} \mathrm{T}$. At low temperatures, the peak position should first increase with temperature (blue-shift). At higher temperatures, the energy gap shrinkage surpasses the increase of $(1 / 2) \mathrm{k}_{\mathrm{B}} \mathrm{T}$ and the peak starts to red-shift.

\subsubsection{Band-to-bound Transitions}

A band-to-bound transition mainly refers to a recombination of a free electron in the conduction band and a hole bound to a neutral acceptor $\left(\mathrm{e}, \mathrm{A}^{0}\right)$, or an electron bound to a neutral donor and a free hole in the valence band $\left(D^{0}, h\right)$. In the first transition, the peak energy of the emission is given by

$$
\mathrm{h} v=\mathrm{E}_{\mathrm{g}}-\mathrm{E}_{\mathrm{A}}+(1 / 2) \mathrm{k}_{\mathrm{B}} \mathrm{T}
$$

where $E_{A}$ is the ionization energy of the acceptor level. In the $\left(D^{0}, h\right)$ transition, the peak energy of the emitted photon is

$$
h v=E_{g}-E_{D}
$$


In most direct-gap semiconductors, the electron effective mass is much smaller than the hole effective mass, hence $E_{D}<E_{A}$, and these two transitions are easy to distinguish. Conceivably, there can be shallow band-to-bound transitions, e.g., a free electron from the CB neutralizing an ionized donor, or a free hole neutralizing an ionized acceptor. However, the probability of such a shallow radiative recombination is several orders lower than that of a non-radiative phonon emission process (Pankove, 1971).

\subsubsection{Donor-acceptor-pair Transitions}

When both donors and acceptors are present in a semiconductor, the donor electrons will be trapped at the acceptor sites leaving both ionized donors $\left(\mathrm{D}^{+}\right)$and acceptors $\left(\mathrm{A}^{-}\right)$ under equilibrium conditions. Under optical excitation with above-band-gap light, the free electrons created in the $\mathrm{CB}$ and free holes in the VB can neutralize these impurities and temporarily convert them back to their neutral states, i.e., $\mathrm{D}^{0}$ and $\mathrm{A}^{0}$. In this excited-state configuration, the weak interaction between the two neutral centers can be described according to a van der Waals coupling term. When the electron bound to the neutral donor recombines with the hole bound to a neutral acceptor, these two centers become ionized once again and thus are subject to a Coulomb attraction. This lowers the energy in the final state. The photon energy resulting from a donor-acceptor pair (DAP) transition is given by (Hopfield, 1963)

$$
h v=E_{g}-E_{D}-E_{A}+\frac{e^{2}}{4 \pi \varepsilon_{r} \varepsilon_{0}} \frac{1}{r}-\frac{e^{2}}{4 \pi \varepsilon_{r} \varepsilon_{0}}\left(\frac{a}{r}\right)^{6}
$$

where $r$ is the spatial separation of the neutral donor and the neutral acceptor involved in a DAP transition. The last term in Eq. 2.16 describes the van der Waals interaction between a neutral donor and a neutral acceptor, and a is the effective van der Waals coefficient. Often, 
this term is assumed negligible in standard treatments of semiconductors. In $\mathrm{ZnO}$, the donor and electron ionization energies can be large and the electron (or hole) wavefunctions are rather localized, so, for DAP recombination to take place, $r$ must be $<100 \AA$.

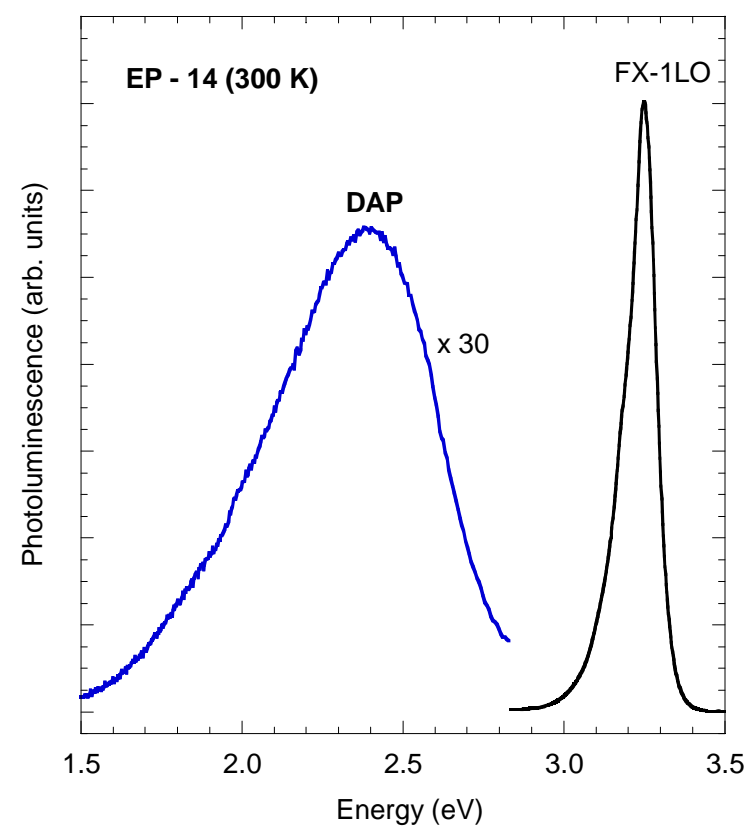

Figure 2.2 PL spectra taken at $300 \mathrm{~K}$ from a single crystal sample prepared by SCVT method at Eagle-Picher Inc. (Miami, OK). The DAP peak is broad and featureless. The sharper and more intense peak at the right is the FX-1LO emission.

Due to the periodic lattice structure, the value of $r$ in the Coulomb interaction term is discrete, hence the energy of emitted photons are discrete. This should give rise to some sharp lines in addition to the DAP peak determined by $h v_{m}=E_{g}-\left(E_{D}+E_{A}\right)$, as have been observed in GaP (Thomas, 1964). As $\mathrm{r}$ increases, the separation between two adjacent sharp lines becomes smaller and smaller till nonresolvable, and the DAP spectrum reaches its lower energy edge, $\mathrm{h} v_{\mathrm{m}}$. The probability for a distant pair to recombine is smaller than that of a closer pair, but the number of possible pairs increases with $r$. Hence, the spectrum will have a maximum at some intermediate value of $r$.

In the literature, DAP recombinations with discrete sharp lines have not been reported from $\mathrm{WZ}$ structure materials such as $\mathrm{ZnO}$. The DAP spectra from $\mathrm{ZnO}$ are usually featureless broad bands. A typical 300-K PL spectrum from single crystal $\mathrm{ZnO}$ is shown in 
Figure 2.2, where the broad DAP peak is in contrast to the sharper FX-1LO line at higher energy. Care must be taken in extrapolating donor and acceptor ionization energies from the DAP band shown here. The broadening of the DAP band is mainly due to phonon-assisted transitions from the excited state to the ground state, and the band has a Poisson distribution shape depending on the Huang-Rhys factor (S). While the energy of the zero phonon-line (ZPL), which is associated with the transition from the minimum of exited state to the ground state, is determined by Equation (2.16), the peak of the DAP band appears below the ZPL by an energy difference of $\Delta \mathrm{E}=\mathrm{Sh} \omega_{\mathrm{LO}}(\mathrm{Fox}, 2001)$. For $\mathrm{ZnO}, \mathrm{S}=6.5$ has been deduced by several groups for this particular band (Dingle, 1969; Kuhnert, 1981).

\subsubsection{Competition between PL and Non-radiative Processes}

After the excitation energy is absorbed by the material, the energy can be released either through PL emissions, as discussed above, or by other competing non-radiative processes, such as the Auger effect, surface recombination, and multiple-phonon emission. In the Auger effect, when an e-h pair combines, the released energy is immediately absorbed by another electron, which then dissipates that energy by emitting phonons. In the surfacerecombination process, the dangling bonds on the sample surface absorb impurities from the ambient and create a continuous distribution of surface states which differ significantly from bulk states. When the electrons and holes are within a diffusion depth of the surface, their recombination through the continuous states is non-radiative. Similarly, a localized defect can produce a microscopic internal surface and cause similar non-radiative recombination, once the electrons and holes are close enough to the trap. Occasionally, the non-radiative recombination can occur by emitting a cascade of phonons whose energy is much smaller than the released energy. 
The probability of a non-radiative recombination process, $\mathrm{P}_{\mathrm{nr}}$, can have a strong temperature dependence. If $\mathrm{P}_{\mathrm{nr}}(0)$ is the probability at liquid helium temperatures, then an expression can be used of the form: $\mathrm{P}_{n r}=\mathrm{P}_{n r}(0) \exp \left(-\mathrm{E}_{\mathrm{A}} / \mathrm{k}_{\mathrm{B}} \mathrm{T}\right)$, where $\mathrm{E}_{\mathrm{A}}$ is the activation energy of the specific non-radiative recombination. Using this form, the temperature dependent quenching of the radiative NBE emission is described as (Pankove, 1971)

$$
I(T)=I(0) \frac{P_{r}}{P_{r}+P_{n r}}=\frac{I(0)}{1+C \exp \left(-E_{A} / k_{B} T\right)}
$$

where $\mathrm{P}_{\mathrm{r}}$ is the probability for radiative emission and is usually assumed independent of temperature. In Eq. 2.17, the constant $C$ is equal to the ratio $P_{n r}(0) / P_{r}$.

\subsection{Temperature Dependent Bandgap and Bandgap Renormalization}

In photoluminescence, the energy of the emitted photon is determined by the transition energy for the given recombinations with respect to the band-gap energy $E_{g}$. The value of $\mathrm{E}_{\mathrm{g}}$ is dependent on temperature and other factors such as free carrier concentration. To describe the temperature-dependence of $E_{g}$, established models include Varshni's equation (Varshni, 1967)

$$
E_{g}(T)=E_{g}(0)+\alpha T^{2} /(T+\beta)
$$

and the Manoogian-Woolley (MW) equation (Manoogian, 1984; Hamby, 2003)

$$
\mathrm{E}_{\mathrm{g}}(\mathrm{T})=\mathrm{E}_{\mathrm{g}}(0)+\mathrm{UT}^{\mathrm{s}}+\mathrm{V} \theta_{\mathrm{E}}\left[\operatorname{coth}\left(\theta_{\mathrm{E}} / 2 \mathrm{~T}\right)-1\right]
$$

where the second term describes the contribution of lattice dilation and the third term describes that of the electron-phonon interaction. There are five fitting parameters in Eq. 2.19. These parameters are the low-temperature band-gap $\mathrm{E}_{\mathrm{g}}(0), \mathrm{U}, \mathrm{s}, \mathrm{V}$ and $\theta$. The value of $\mathrm{U}$ is expected to be close to $-3 \mathrm{~B}\left(\partial \mathrm{E}_{\mathrm{g}} / \partial \mathrm{P}\right)<\alpha \mathrm{L}>$, where $\mathrm{B}$ is the bulk modulus, $\left(\partial \mathrm{E}_{\mathrm{g}} / \partial \mathrm{P}\right)$ is the bandgap pressure coefficient, and $<\alpha \mathrm{L}>$ is the mean thermal expansion coefficient. The 
value of $\mathrm{s}$ is often found close to 1 . The second term is often found to contribute about $15 \%$ of the band-gap shrinkage. In the third term, $\theta_{\mathrm{E}}$ is the effective Einstein phonon temperature for the material. As has been shown in group IV semiconductors, the value of $\theta_{\mathrm{E}}$ corresponds to the mean value of respective acoustic and optical phonons (Walter, 1970).

In Eq. 2.19, by defining $X=\theta_{E} / 2 T$, the second term could be written as $V \theta_{E}$ $[2 /(\exp 2 \mathrm{X}-1)]$. For $2 \mathrm{X}<<1, \exp 2 \mathrm{X} \approx 1+2 \mathrm{X}+2 \mathrm{X}^{2}, \mathrm{~V} \theta_{\mathrm{E}}[2 /(\exp 2 \mathrm{x}-1)] \approx \mathrm{V} \theta_{\mathrm{E}}\left[1 /\left(\mathrm{X}+\mathrm{X}^{2}\right)\right]=$ $2 \mathrm{VT}^{2} /\left(\mathrm{T}+\theta_{\mathrm{E}} / 2\right)=\alpha \mathrm{T}^{2} /(\mathrm{T}+\beta)$, with $\alpha=2 \mathrm{~V}$ and $\beta=\theta_{\mathrm{E}} / 2$. In other words, Varshni's equation is equivalent to the MW equation for high temperatures (i.e., $\mathrm{T}>>\theta_{\mathrm{E}}$ ) if one ignores the lattice-dilation contribution to band-gap shrinkage.

Eq. 2.19 sets up a limit of $E_{g}(T)$ for ideal, or very pure, samples. In real samples, the existence of impurities and free carriers could affect the band-gap and cause further renormalization of $\mathrm{E}_{\mathrm{g}}(\mathrm{T})$. Two effects related to free carriers contribute to the shrinkage of the minimum electronic band-gap in a semiconductor (Bugajski, 1985): the band-tailing effect due to the electron-ionized-impurity interaction through screened Coulomb potentials, and the exchange interaction between free carriers.

$$
E_{g}=E_{g}(0)-E_{c}^{c^{*}}-E_{c}^{e}
$$

where $E_{g}(0)$ is the band-gap in the limit of no carriers, $E_{c}^{c^{*}}$ is the band tailing due to the Coulomb interaction between electron and impurities, and $E_{c}^{e}$ is the band-gap shrinkage due to exchange interactions between free carriers.

$$
\begin{aligned}
& E_{c}^{c^{*}}=E_{c}^{c}\left(1-2 \alpha \frac{E_{F}^{0}}{E_{g}}\right) \\
& E_{c}^{c}=\left(\frac{\pi^{4 / 3} \hbar^{2}}{3^{1 / 3}}\right)\left(\frac{1}{m^{*}}\right) n^{2 / 3}
\end{aligned}
$$




$$
E_{c}^{e}=\left(\frac{e}{2 \pi \varepsilon_{r} \varepsilon_{0}}\right)\left(\frac{3}{\pi}\right)^{1 / 3} n^{1 / 3}
$$

Here $E_{F}^{0}$ is the Fermi energy at a given free carrier level,

$$
E_{F}^{0}=\left(\frac{\hbar^{2}}{2 m_{0}{ }^{*}}\right)\left(3 \pi^{2} n\right)^{2 / 3}
$$

and the parameter $\alpha$ is the non-parabolicity coefficient for the conduction band used in the correction of the electron effective mass for nonzero $k$ values. In general,

$$
m^{*}=m_{0}\left(1-\frac{2 \alpha E_{F}^{0}}{E_{g}}\right)^{-1}
$$

For $\mathrm{ZnO}, \alpha$ has the assumed form of (Bai, 2005)

$$
\begin{aligned}
& \alpha=\left(1-\frac{m_{0}{ }^{*}}{m_{0}}\right)^{2}\left[-\frac{6+4 \frac{E_{1}}{E_{g}}-2 \frac{E_{2}}{E_{g}}}{\left(1+\frac{E_{1}}{E_{g}}\right)^{2}+2\left(1+\frac{E_{1}}{E_{g}}-\frac{E_{2}}{E_{g}}\right)-\frac{2}{3} \frac{\Delta_{s o}}{E_{g}} \frac{\Delta_{c f}}{E_{g}}}\right. \\
& \left.+\frac{\left(1+\frac{E_{1}}{E_{g}}\right)^{2}+2\left(1+\frac{E_{1}}{E_{g}}-\frac{E_{2}}{E_{g}}\right)-\frac{E_{1}}{E_{g}} \frac{E_{2}}{E_{g}}}{\left(1+\frac{E_{1}}{E_{g}}\right)\left(1+\frac{E_{1}}{E_{g}}-\frac{E_{2}}{E_{g}}\right)}\right]
\end{aligned}
$$

with $\mathrm{E}_{1,2}=-(1 / 2)\left(\Delta_{\mathrm{so}}+\Delta_{\mathrm{cf}}\right) \pm(1 / 2)\left[\left(\Delta_{\mathrm{so}}+\Delta_{\mathrm{cf}}\right)^{2}-(8 / 3)\left(\Delta_{\mathrm{so}} * \Delta_{\mathrm{cf}}\right)\right]^{1 / 2}$. Here, $\Delta_{\mathrm{so}}$ is the spinorbit energy splitting and $\Delta_{\mathrm{cf}}$ is the crystal-field energy splitting. The energy values appropriate for $\mathrm{ZnO}$ are $\Delta_{\mathrm{so}}=-0.008 \mathrm{eV}$, and $\Delta_{\mathrm{cf}}=0.0417 \mathrm{eV}$, leading to $\mathrm{E}_{1}=0.0057 \mathrm{eV}=$ splitting between valence bands $\mathrm{A}$ and $\mathrm{B}$, and $\mathrm{E}_{2}=-0.0393 \mathrm{eV}=$ splitting between valence bands $\mathrm{B}$ and $\mathrm{C}$.

For $\mathrm{ZnO}$, using an electron effective mass of $\mathrm{m}^{*}=0.24 \mathrm{~m}_{0}$, the numerical value of nonparabolicity coefficient can be determined as $\alpha=(1-0.24)^{2}[-(6.0301 / 3.0301)+$ $(3.0301 / 1.0151)]=0.575$. The numerical expressions for the remaining energy parameters 
needed to describe the band-gap energy and defined in Eqs. (2.22-24) are given below (energy is in $\mathrm{eV}$ and $\mathrm{n}$ is in $\mathrm{cm}^{-3}$ ):

$$
\begin{aligned}
E_{F}^{0} & =\left(1.055 \times 10^{-34}\right)^{2} /\left(2 \times 0.91 \times 10^{-30} \times 1.60 \times 10^{-19}\right)\left(3 \pi^{2}\right)^{2 / 3} \mathrm{n}^{2 / 3} /\left(\mathrm{m}^{*} / \mathrm{m}_{0}\right) \\
& =3.656 \times 10^{-15}\left(\mathrm{~m}_{0} / \mathrm{m}^{*}\right) \mathrm{n}^{2 / 3}=1.52 \times 10^{-14} \mathrm{n}^{2 / 3} ; \\
E_{c}^{c} & =\left(2 \pi^{4 / 3} / 3^{1 / 3}\right)\left(\hbar^{2} / 2 \mathrm{~m}_{0}\right)\left(\mathrm{m}_{0} / \mathrm{m}^{*}\right) \mathrm{n}^{2 / 3}=1.02 \times 10^{-14} \mathrm{n}^{2 / 3} ; \\
E_{c}^{e} & =4 \mathrm{r}_{\mathrm{H}}(13.6 \mathrm{eV})(3 / \pi)^{1 / 3}\left(1 / \varepsilon_{\mathrm{r}}\right) \mathrm{n}^{1 / 3}=3.64 \times 10^{-8} \mathrm{n}^{1 / 3}, \text { when using } \varepsilon_{\mathrm{r}}=7.8 .
\end{aligned}
$$

Besides the band-tailing effect and the exchange interaction, the optical band-gap $\left(E_{o p t}\right)$ will be affected by the Fermi level, which defines the available empty states in the conduction band

$$
E_{\text {opt }}=E_{g}(0)-E_{c}^{c^{*}}-E_{c}^{e}+E_{F}^{*}
$$

where

$$
E_{F}^{*}=E_{F}^{0}\left(1-\alpha \frac{E_{F}^{0}}{E_{g}}\right)
$$

In the case of high n-type (or high p-type) material, the momentum conservation during absorption requires the consideration of the valence band dispersion, and $E_{F}{ }^{*}$ should be replaced with $\left(1+\mathrm{m}_{\mathrm{e}}{ }^{*} / \mathrm{m}_{\mathrm{p}}{ }^{*}\right) E_{\mathrm{F}}{ }^{*}$, where $\mathrm{m}_{\mathrm{e}}{ }^{*}$ and $\mathrm{m}_{\mathrm{p}}{ }^{*}$ are electron and hole effective masses, respectively. For $\mathrm{ZnO}, \mathrm{m}_{\mathrm{e}}{ }^{*} / \mathrm{m}_{\mathrm{p}}{ }^{*}=0.24 / 0.45=0.53$, and the last term in Eq. 2.27 becomes $1.53 E_{F}^{*}$.

\subsection{Hall Effect}

In the presence of both electric and magnetic fields (see Figure 2.3), the equation of motion of a free electron in a sample can be written as 


$$
\mathrm{m}^{*}\left(\mathrm{~d}^{2} \mathbf{r} / \mathrm{dt}^{2}\right)+\left(\mathrm{m}^{*} / \tau\right) \mathrm{d} \mathbf{r} / \mathrm{dt}=(-\mathrm{e})(\mathbf{E}+\mathbf{v} \times \mathbf{B})
$$

where $\tau$ is the relaxation time. In steady state, i.e., $\mathrm{dv} / \mathrm{dt}=\mathrm{d}^{2} \mathbf{r} / \mathrm{dt}^{2}=0$, the above equation becomes

$$
\left(\mathrm{m}^{*} / \tau\right) \mathbf{v}_{\mathrm{d}}=(-\mathrm{e})(\mathbf{E}+\mathbf{v} \times \mathbf{B})
$$

where $\mathbf{v}_{\mathrm{d}}$ is the drift velocity.

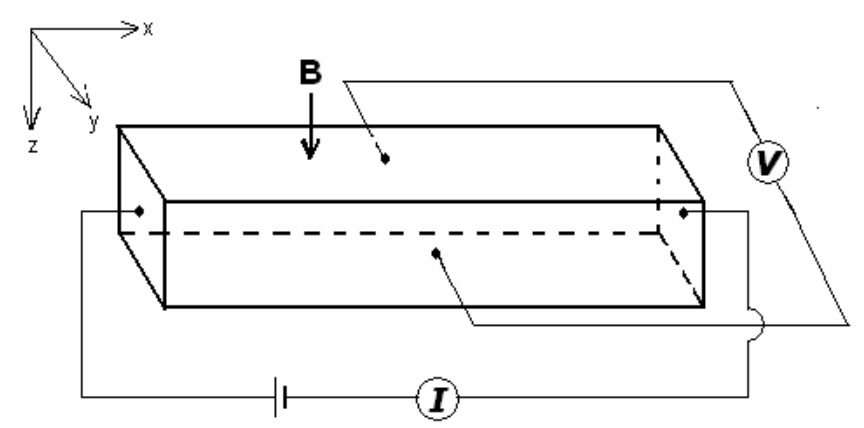

Figure 2.3 A schematic sketch of a Hall effect measurement.

By defining the current density as $\mathbf{j}=n(-\mathrm{e}) \mathbf{V}_{\mathrm{d}}$, and assuming $\mathbf{B}=\left(0,0, \mathrm{~B}_{\mathrm{z}}\right)$ and an arbitrary direction of $\mathbf{E}$, Eq. (2.30) can be rewritten as

$$
\begin{aligned}
& j_{x}=\sigma_{0} E_{x}-\omega_{c} \tau j_{y}, \\
& j_{y}=\sigma_{0} E_{y}+\omega_{c} \tau j_{x}, \\
& j_{z}=\sigma_{0} E_{z} .
\end{aligned}
$$

Solving the equations one obtains

$$
\begin{aligned}
& \mathrm{j}_{\mathrm{x}}=\sigma_{0}\left(\mathrm{E}_{\mathrm{x}}-\omega_{\mathrm{c}} \tau \mathrm{E}_{\mathrm{y}}\right) /\left[1+\left(\omega_{\mathrm{c}} \tau\right)^{2}\right], \\
& \mathrm{j}_{\mathrm{y}}=\sigma_{0}\left(\mathrm{E}_{\mathrm{y}}+\omega_{\mathrm{c}} \tau \mathrm{E}_{\mathrm{x}}\right) /\left[1+\left(\omega_{\mathrm{c}} \tau\right)^{2}\right], \\
& \mathrm{j}_{\mathrm{z}}=\sigma_{0} \mathrm{E}_{\mathrm{z}} .
\end{aligned}
$$

where $\sigma_{0}=\mathrm{ne}^{2} \tau / \mathrm{m}^{*}$ is the conductivity when $\mathrm{B}=0$, and $\omega_{\mathrm{c}}=\mathrm{eB}_{\mathrm{z}} / \mathrm{m}^{*}$ is the classic cyclotron frequency of the electron in the presence of a magnetic field B. In a simplified case, say, the applied electric field is along $\mathrm{x}$ direction, the Lorentz force will cause an additional $\mathrm{j}_{\mathrm{y}}$ which 
results in accumulated electric voltage and an induced electronic field $\mathrm{E}_{\mathrm{y}}$. A new steady state is reached when this field cancels the Lorentz force on electrons and $\mathrm{j}_{\mathrm{y}}=0$. In the low-field limit $\left(\omega_{\mathrm{c}} \tau<<1\right)$, this leads to $\mathrm{j}_{\mathrm{x}}=\sigma_{0} \mathrm{E}_{\mathrm{x}}$, and $\mathrm{E}_{\mathrm{y}}=-\omega_{\mathrm{c}} \tau \mathrm{E}_{\mathrm{x}}=-\omega_{\mathrm{c}} \tau \mathrm{j}_{\mathrm{x}} / \sigma_{0}$. This phenomenon is known as the Hall effect, which was discovered by E. H. Hall in 1879 . The induced electric field $E_{y}$ is connected with the control parameters $B_{z}$ and $E_{x}$ through the introduction of the Hall coefficient, $R_{H}=E_{y} / B_{z} j_{x}$. However, $E_{y} / j_{x}=-\omega_{c} \tau / \sigma_{0}=-\left(e B_{z} \tau / m^{*}\right) /\left(n e^{2} \tau / m^{*}\right)=-B_{z} / n e$, hence $\mathrm{R}_{\mathrm{H}}=-1 /$ ne, for $\mathrm{n}$-type samples. In $\mathrm{p}$-type material, the accumulation of holes induces an electric field $\mathrm{E}_{\mathrm{y}}=\omega_{\mathrm{c}} \tau \mathrm{E}_{\mathrm{x}}$, and $\mathrm{R}_{\mathrm{H}}=1 /$ pe is positive. Thus, the sign of $\mathrm{R}_{\mathrm{H}}$ is a way to tell whether the majority free carriers in a sample are electrons or holes. In the presence of both electrons (of concentration $n$ and mobility $\mu_{\mathrm{n}}$ ) and holes (of concentration $\mathrm{p}$ and $\mu_{\mathrm{p}}$ ) in the same sample, the conductivity is given by $\sigma=\mathrm{e}\left(\mathrm{n} \mu_{\mathrm{n}}+\mathrm{p} \mu_{\mathrm{p}}\right)$, and the low-field Hall coefficient has the form

$$
R_{H}=\frac{p-b^{2} n}{e(p+b n)^{2}}
$$

where $b=\mu_{\mathrm{n}} / \mu_{\mathrm{p}}$ is the ratio of electron and hole mobilities.

\subsubsection{Hall $r$ factor}

The discussion above ignored the energy variation and distribution of electrons in a real sample. When the range of electron energy $E$ and a distribution function $\mathrm{f}(E)$ are considered, any statistical property $\mathrm{P}(E)$ of electrons in a sample is calculated as an average denoted by $<\mathrm{P}(E)>$, where

$$
\langle P(E)\rangle=\int P(E) f(E) d E / \int f(E) d E
$$


In Eq. (2.31), due to the energy dependence of the relaxation time, $\tau=\tau(E)$, the three components of $\mathbf{j}$ can be expressed as average forms according to

$$
\begin{aligned}
& <j_{x}>=\alpha E_{x}-\gamma B_{z} j_{y}, \\
& <j_{y}>=\alpha E_{y}+\gamma B_{z} j_{x}, \\
& <j_{z}>=<\sigma 0>E_{z}
\end{aligned}
$$

where

$$
\begin{aligned}
& \alpha=\frac{n e^{2}}{m^{*}}\left\langle\frac{\tau}{1+\left(\omega_{c} \tau\right)^{2}}\right\rangle \approx \frac{n e^{2}}{m^{*}}\langle\tau\rangle \\
& \gamma=\frac{n e^{3}}{m^{* 2}}\left\langle\frac{\tau^{2}}{1+\left(\omega_{c} \tau\right)^{2}}\right\rangle \approx \frac{n e^{3}}{m^{* 2}}\left\langle\tau^{2}\right\rangle
\end{aligned}
$$

The Hall coefficient for the corresponding energy distribution of electrons can be written as

$$
R_{H}=\frac{\left\langle\tau^{2}\right\rangle}{(-n e)\langle\tau\rangle^{2}}=-\frac{r_{H}}{n e}
$$

where $r_{H}=\left\langle\tau^{2}>/<\tau\right\rangle^{2}$ is called the Hall $\mathrm{r}$ factor. The Hall $\mathrm{r}$ factor relates the Hall mobility $\mu_{\mathrm{H}}$ with the conductivity mobility $\mu$ according to $\mu_{\mathrm{H}}=R_{\mathrm{H}} \sigma_{0}=\left(-\sigma_{0} / \mathrm{ne}\right) \mathrm{r}_{\mathrm{H}}=\mathrm{r}_{\mathrm{H}} \mu$. Correspondingly, the real free carrier concentration $\mathrm{n}$ is connected with the measured Hall carrier concentration $\mathrm{n}_{\mathrm{H}}$ as $\mathrm{n}_{\mathrm{H}}=\mathrm{n} / \mathrm{r}_{\mathrm{H}}$.

In the non-degenerate electron gas approximation, the Boltzmann distribution is applied, i.e., $\mathrm{f}(E) \sim \exp \left(-E / \mathrm{k}_{\mathrm{B}} \mathrm{T}\right)$. For a three dimensional parabolic band, the density-ofstates is

$$
D(E)=\frac{1}{2 \pi}\left(\frac{2 m^{*}}{\hbar^{2}}\right)^{3 / 2} E^{1 / 2}
$$

If we assume the relaxation time has temperature and energy dependence of the form $\tau=$ $\tau(\mathrm{T}, E) \sim \mathrm{T}^{\mathrm{m}} E^{\mathrm{n}}$, then 


$$
\begin{aligned}
& \int E^{3 / 2} \exp \left(-E / \mathrm{k}_{\mathrm{B}} \mathrm{T}\right) \mathrm{d} E \sim\left(\mathrm{k}_{\mathrm{B}} \mathrm{T}\right)^{5 / 2}[\Gamma(5 / 2)] \\
& \int \tau(\mathrm{T}, E) E^{3 / 2} \exp \left(-E / \mathrm{k}_{\mathrm{B}} \mathrm{T}\right) \mathrm{d} E \sim\left(\mathrm{k}_{\mathrm{B}} \mathrm{T}\right)^{\mathrm{m}+\mathrm{n}+5 / 2}[\Gamma(\mathrm{n}+5 / 2)] \\
& \int(\tau(\mathrm{T}, E))^{2} E^{3 / 2} \exp \left(-E / \mathrm{k}_{\mathrm{B}} \mathrm{T}\right) \mathrm{d} E \sim\left(\mathrm{k}_{\mathrm{B}} \mathrm{T}\right)^{2 \mathrm{~m}+2 \mathrm{n}+5 / 2}[\Gamma(2 \mathrm{n}+5 / 2)]
\end{aligned}
$$

Hence, for the mobility associated with a particular scattering mechanism, the temperature dependence is determined by the form of $<\tau>$.

$$
\begin{aligned}
\mu(\mathrm{T}) & =\left(\mathrm{e} / \mathrm{m}^{*}\right)<\tau> \\
& =\left(\mathrm{e} / \mathrm{m}^{*}\right) \int \tau(\mathrm{T}, E) E^{3 / 2} \exp \left(-E / \mathrm{k}_{\mathrm{B}} \mathrm{T}\right) \mathrm{d} E / \int E^{3 / 2} \exp \left(-E / \mathrm{k}_{\mathrm{B}} \mathrm{T}\right) \mathrm{d} E \\
& \sim\left(\mathrm{k}_{\mathrm{B}} \mathrm{T}\right)^{\mathrm{m}+\mathrm{n}}[\Gamma(\mathrm{n}+5 / 2)] /[\Gamma(5 / 2)]
\end{aligned}
$$

The Hall $\mathrm{r}$ factor is written as

$$
\begin{aligned}
\mathrm{r}_{\mathrm{H}} & =\left\langle\tau^{2}>/\langle\tau\rangle^{2}\right. \\
= & {\left[\int(\tau(\mathrm{T}, E))^{2} E^{3 / 2} \exp \left(-E / \mathrm{k}_{\mathrm{B}} \mathrm{T}\right) \mathrm{d} E / \int E^{3 / 2} \exp \left(-E / \mathrm{k}_{\mathrm{B}} \mathrm{T}\right) \mathrm{d} E\right] / } \\
& \quad\left[\int \tau(\mathrm{T}, E) E^{3 / 2} \exp \left(-E / \mathrm{k}_{\mathrm{B}} \mathrm{T}\right) \mathrm{d} E / \int E^{3 / 2} \exp \left(-E / \mathrm{k}_{\mathrm{B}} \mathrm{T}\right) \mathrm{d} E\right]^{2} \\
& =[\Gamma(2 \mathrm{n}+5 / 2) / \Gamma(5 / 2)] /[\Gamma(\mathrm{n}+5 / 2) / \Gamma(5 / 2)]^{2} \\
= & \Gamma(2 \mathrm{n}+5 / 2) \Gamma(5 / 2)] /[\Gamma(\mathrm{n}+5 / 2)]^{2}
\end{aligned}
$$

As two examples, acoustic phonon scattering via the deformation potential gives $\tau=$ $\tau(\mathrm{T}, E) \sim 1 /\left(\mathrm{T} E^{1 / 2}\right)$, hence $\mathrm{m}=-1, \mathrm{n}=-1 / 2$, and $\mu(\mathrm{T}) \sim \mathrm{T}^{-3 / 2}$, while the acoustic phonon scattering via the piezoelectric potential gives $\tau \sim E^{1 / 2} / \mathrm{T}$, hence $\mathrm{m}=-1, \mathrm{n}=1 / 2$, and $\mu \sim \mathrm{T}^{-1 / 2}$. For the Hall $\mathrm{r}$ factor, the deformation potential scattering gives

$$
\left.\mathrm{r}_{\mathrm{H}}{ }^{\mathrm{dp}}=\Gamma(-1+5 / 2) \Gamma(5 / 2)\right] /[\Gamma(-1 / 2+5 / 2)]^{2}=\Gamma(3 / 2) \Gamma(5 / 2) /[\Gamma(2)]^{2}=3 \pi / 8 \approx 1.18
$$

and the piezoelectric scattering gives a slightly lower value

$$
\left.\mathrm{r}_{\mathrm{H}}^{\mathrm{pe}}=\Gamma(1+5 / 2) \Gamma(5 / 2)\right] /[\Gamma(1 / 2+5 / 2)]^{2}=\Gamma(7 / 2) \Gamma(5 / 2) /[\Gamma(3)]^{2}=45 \pi / 128 \approx 1.10 .
$$




\subsubsection{Hall measurements using the van der Pauw method}

In Hall effect measurements, if the sample has a geometry of rectangular bar (Figure 2.3), the controlling and induced parameters, $\mathrm{j}_{\mathrm{x}}, \mathrm{B}_{\mathrm{z}}$, and $\mathrm{E}_{\mathrm{y}}$, are well defined, and the analysis is relatively straightforward. In cases where a regular Hall bar of the sample is not available, such as with a thin film sample, an extension to the Hall technique has been developed by van der Pauw (van der Pauw, 1958). As shown in Fig. 2.4(a), four contacts are made along the peripheral of a sample of arbitrary shape. With magnetic field off, the resistivity $\rho$ is measured using

$$
\rho=\frac{\pi d f}{\ln 2}\left[\frac{R_{21,34}+R_{32,41}}{2}\right]
$$

where $d$ is sample thickness, $f$ is a slow-varying function of the ratio of $R_{21,34} / R_{32,41}$, or $R_{32,41} / R_{21,34}$, whichever is greater than unity. If the ratio is exactly 1 , then $f=1$. The resistance $R_{21,34}$ is calculated by first applying a constant DC current $\left(I_{1}\right)$ between contacts 1 and 2, and measuring the voltage between 3 and $4\left(\mathrm{~V}_{1}\right)$, then reversing the direction of current $\left(\mathrm{I}_{2}\right)$ and measuring voltage again $\left(\mathrm{V}_{2}\right)$. The resistance is the average of the two measurements:
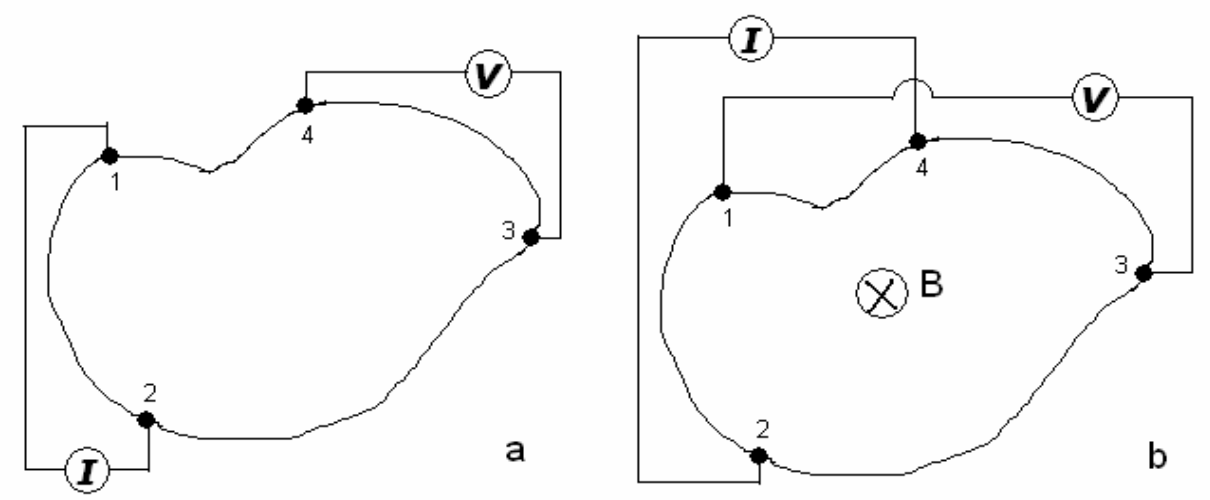

Figure 2.4 Schematic sketch of Hall effect measurements using a van der Pauw geometry, (a) without magnetic field, and (b) with magnetic field perpendicular to sample plate. 


$$
\mathrm{R}_{21,34}=\left(\mathrm{V}_{1}-\mathrm{V}_{2}\right) /\left(\mathrm{I}_{1}-\mathrm{I}_{2}\right)
$$

When a magnetic field is applied, a different combination of contacts is used for current and voltage measurements, as shown in Fig. 2.4(b). The Hall coefficient is calculated using

$$
R_{H}=\frac{d}{B}\left[\frac{R_{31,24}+R_{24,31}}{2}\right]
$$

From Eqs. 2.45 and 2.47, the Hall mobility is obtained using $\mu_{H}=R_{H} / \rho$, and the Hall free carrier concentration is given by $n_{H}=1 /\left(e R_{H}\right)$.

For small sample sizes, the actual sizes of the ohmic contacts relative to the dimensions of the sample must be considered. This was first theoretically analyzed by van der Pauw and later studied by others (Chwang, 1974). The finite contact size causes a drop in measured sample resistivity and thus a drop in Hall voltage. For example, if a sample has a disc shape with diameter $l_{\mathrm{s}}$, and the contact dimension (e.g., diameter for a dot-shape contact, or the side length for a square-shape contact) is $l_{\mathrm{c}}$, the relative loss in $\mathrm{R}_{\mathrm{H}}$ and $\mu_{\mathrm{H}}$ was estimated as (van der Pauw, 1958)

$$
\frac{\Delta \mu_{H}}{\mu_{H}}=\frac{\Delta R_{H}}{R_{H}} \approx \frac{2}{\pi^{2}} \frac{l_{c}}{l s}
$$

Chwang et al. showed that this could be worse for low-field measurements, where $\mu \mathrm{B}$ is small (Chwang, 1974) 


\section{Chapter 3 Experimental Details}

\subsection{Photoluminescence}

Photoluminescence (PL) experiments using above-band-gap laser excitation were carried out using either of two setups. One setup uses a monochromator/PMT detector system, while the other uses a fiber-coupled CCD detector system. Selective-excitation PL was performed using a Xenon lamp coupled into a small monochromator as a tunable broadband white-light source. These experimental set-ups will be described below. A typical above-band-gap excitation PL setup used in this work is shown in Figure 3.1. The 325-nm light is provided by a continuous-wave (cw) Helium-Cadmium laser (Liconix, Model 3225N). The laser beam height is raised by a pair of UV-enhanced reflective aluminum mirrors (M1/M2) to match the height of sample, monochromator slits, and detector. The power of the incident light is controlled using a neutral-density (ND) filter. A band-pass (BP) filter is used to suppress unwanted plasma lines from the laser. A third UV mirror (M3) directs the laser beam to strike the sample at an incident angle of about 20 degree. In experiments

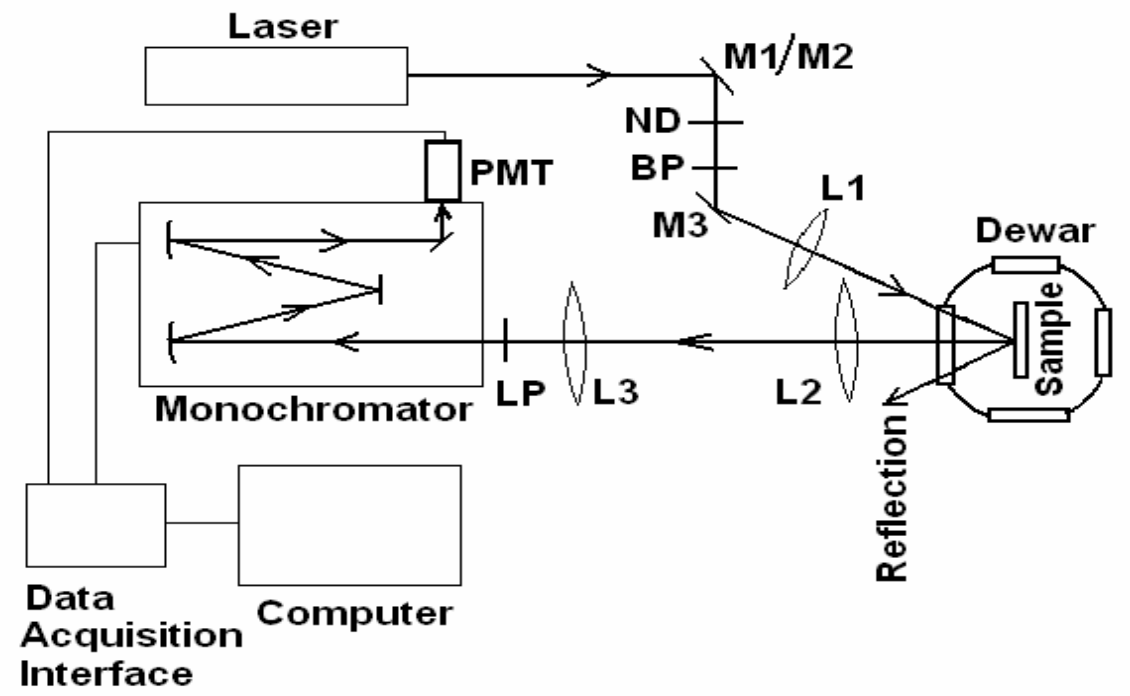

Figure 3.1. PL setup for above band-gap excitation experiments. 
requiring a focused laser beam, $\mathrm{a} \mathrm{CaF}_{2}$ focusing lens (L1) directs the beam onto the sample surface with a light-spot diameter of about $1 / 4 \mathrm{~mm}$. The PL emission is collected with a $\mathrm{CaF}_{2}$ lens (L2) in a back-scattering geometry. The third $\mathrm{CaF}_{2}$ lens (L3) focuses the PL emission onto the entrance slit of the monochromater. A 340-nm long-pass (LP) filter is placed right before the entrance slit to prevent the scattered laser light from entering the monochromator. For scanning wavelengths far below the band gap of $\mathrm{ZnO}$, a $600 \mathrm{~nm} \mathrm{LP}$ filter is used to avoid the second-order diffraction peaks of the scattered laser light and the UV PL emission.

The collected PL emission is dispersed by a 0.64-m monochromator (Instruments SA, Inc., model HR-640) and detected by a photomultiplier tube (PMT) (Hamamatsu, model R943-02) utilized in photon-counting mode. The diffraction grating in the HR-640 has 1200 grooves per $\mathrm{mm}$, and is blazed at $750 \mathrm{~nm}$. The grating position and the signal collection from the PMT are controlled through a computer interface (Spectra Link) using a Windowsbased commercial software program called SpectraMax (from Horiba Jobin-Yvon). The wavelength calibration of the $\mathrm{HR}-640$ monochromator is performed using a $\mathrm{Hg}(\mathrm{Ar})$ pencil lamp. When needed, the monochromator slits were reduced giving a system band-pass of approximately $0.3 \AA$. The wavelength-dependent system detection response is calibrated using a quartz tungsten halogen (QTH) lamp as a white light source (Oriel QTHL-20W, Model 6319). In the wavelength-to-energy conversion, the relation $E=h v / \lambda$ is used, where $v$ is the light speed in air $(\mathrm{v}=\mathrm{c} / \mathrm{n}, \mathrm{n}$ is index of refraction of air). The measured PL signal is recorded as emission intensity vs. wavelength. In many cases, emission intensity vs. energy is needed. The original data, having the form of $\mathrm{I}(\lambda)$, are converted to $\mathrm{I}(\mathrm{E})$ by considering $\mathrm{I}(\mathrm{E}) \mathrm{dE}=\mathrm{I}(\lambda)(-\mathrm{d} \lambda)$, which gives $\mathrm{I}(\mathrm{E})=\mathrm{I}(\lambda) /(-\mathrm{dE} / \mathrm{d} \lambda)=\mathrm{I}(\lambda) \lambda^{2} /(\mathrm{hv})$. 
For more time-efficient recording of room temperature PL data, a second PL setup was used. This second set-up includes a fiber-coupled Imaging Spectrograph system (Oriel Instruments, Model MS260i) equipped with a CCD detector. Luminescence is excited using the 325-nm laser and a ND filter is used control the incident excitation power on the sample surface. The PL emission in either reflective or transmission geometries passed through a LP filter and was then collected by and transferred through a 2-meter long optic fiber to the spectrograph/CCD detector system. The fiber is a bundled high-grade fused silica fiber (Oriel 77532) that provides transmittance of $50-60 \%$ in the range of $300-1100 \mathrm{~nm}$. The collection end has a circular termination with a diameter of $2 \mathrm{~mm}$, and the output end has a rectangular slit of $200 \mu \mathrm{m} \times 6 \mathrm{~mm}$. With the smallest CCD slit opening (12 units, or 30 microns), this fiber-coupled CCD detecting system has a band-pass of $2 \mathrm{~nm}$. A commercial software (InstaSpec IV) was used in data analysis, and data acquisition could be either in real-time mode (e.g., for optical-path optimization), or in accumulative mode (for better signal/noise ratio). This setup allows for fast data collection. The entire wavelength sampling range is accumulated, rather than a scanning system which takes point-by-point spectra. The faster accumulation was particularly useful in this study when large numbers of powder or ceramic $\mathrm{ZnO}$ samples were provided by ORNL and sample-to-sample comparisons, rather than accurate energy resolution, were required.

For selective-excitation PL, the light source is a 150-W short-arc Xenon lamp (Oriel). As shown in Fig. 3.2, the light beam is coupled into a 0.22-m monochromator (SPEX MINIMATE-2 1681b) which is controlled by the Compudrive (SPEX CD2A). The combination of two $\mathrm{CaF}_{2}$ lenses and a UV mirror focuses the dispersed incident light onto the sample with a light spot size of $3 \times 5 \mathrm{~mm}^{2}$. 


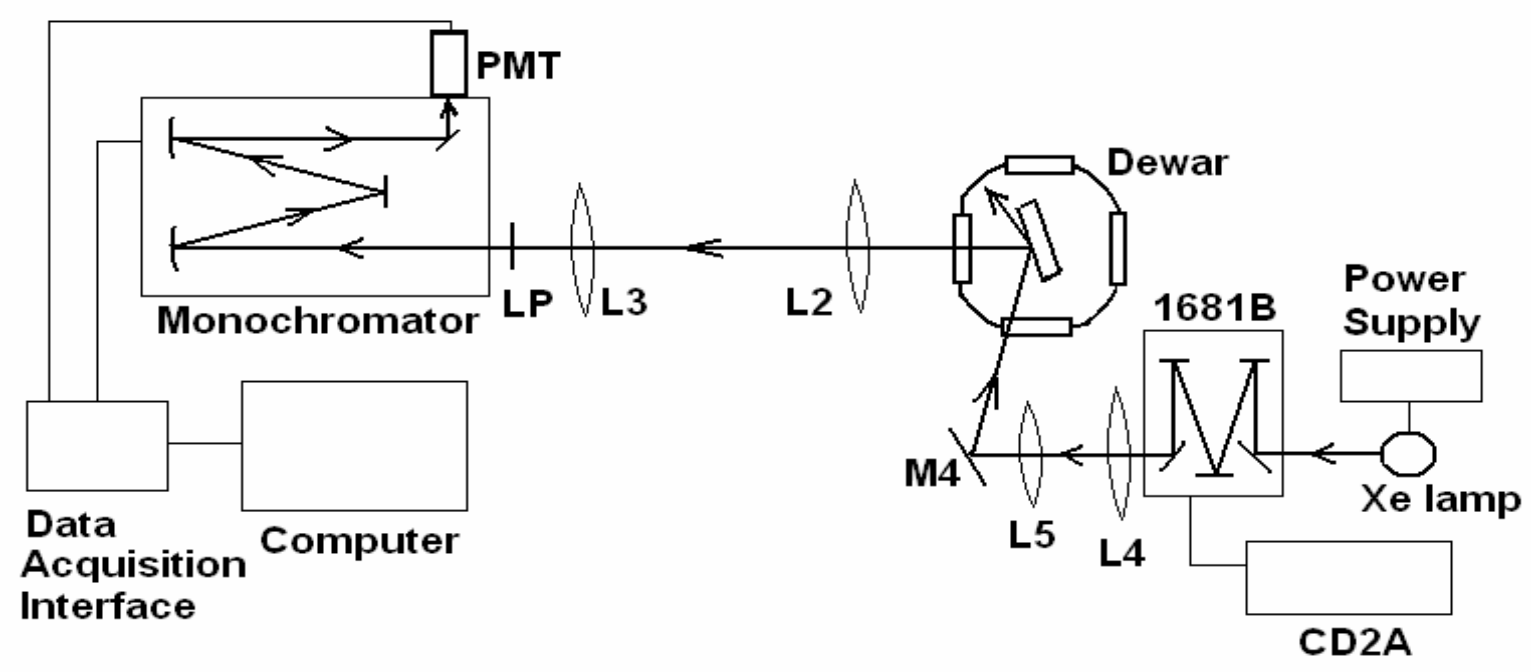

Figure 3.2. PL setup for selective-excitation experiments.

Much of the screening of samples for scintillator applications required only roomtemperature PL experiments. However, in order to understand the recombination paths, low temperature measurements were required using liquid nitrogen or liquid helium. For low temperature PL or temperature-dependent PL, samples are mounted using rubber cement on a molybdenum plate and then attached to a copper plate at the lower part of a sample pole in the sample chamber of an optical dewar (Janis SuperVaritemp 8DT). Sample temperature is monitored using a Si diode temperature sensor (Lake Shore Cryotronics). The cryogenic liquid is introduced into the sample chamber through a needle valve and a capillary tube from the liquid reservoir. A desired temperature between 5 and $300 \mathrm{~K}$ is obtained and maintained by controlling the liquid flow and using a heater coil. A Lake Shore Temperature Controller (Model 805) monitored sample temperature and also allowed control of the current through the heater coil. 


\subsection{X-ray-induced Luminescence}

X-ray induced luminescence (radioluminescence) more closely mimics the highenergy scintillation conditions in the alpha-particle detector as compared with PL using a laser beam of just a few eV. The X-ray source is an industrial tungsten X-ray tube (Varian, Model OEG-76H), controlled by a high voltage power supply (Spellman, Model FF4), with electric current up to $30 \mathrm{~mA}$ and field voltage up to $60 \mathrm{kV}$. The cathode-jacket water-cooling is provided by a circulating chiller (Lytron, Model RC022). The X-ray tube has a circular beryllium window with a diameter of 1 inch, and in X-ray induced luminescence experiments it was confined by lead shield to allow an output beam with a diameter of $1 \mathrm{~cm}$. Figure 3.3 shows schematically the X-ray luminescence setup using reflective and transmission geometries. The luminescence was collected by the fiber head and transferred to the CCD detector coupled with the Spectrograph system (Oriel 260i) for analysis. To protect the fiber from the X-ray, the front end of the fiber head is kept $2 \mathrm{~cm}$ above the incident X-ray beam.

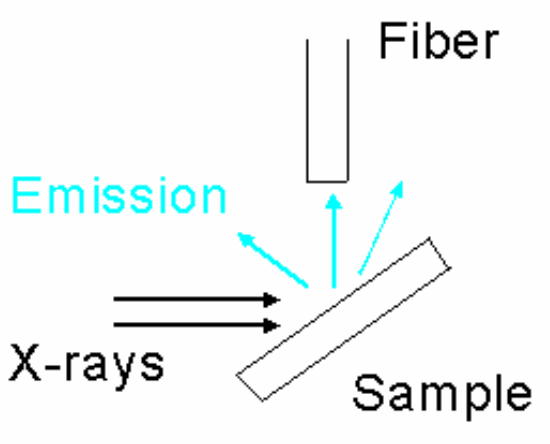

(a)

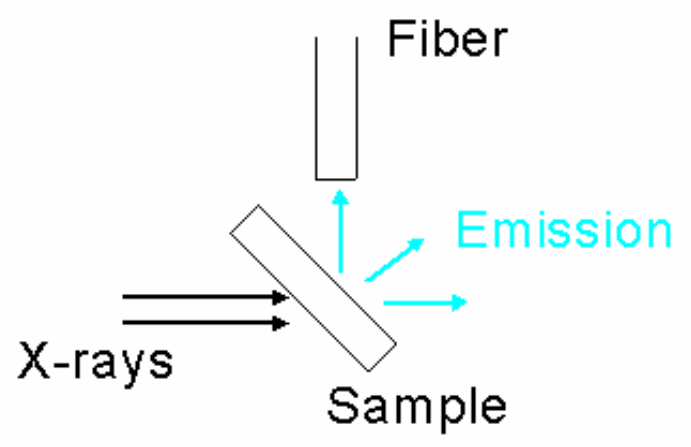

(b)

Figure 3.3 Sketch of X-ray excited luminescence experiment setup using (a) reflective collection and (b) transmission collection geometries.

\subsection{Hall Effect}

Low field Hall effect measurements are carried out with a commercial van der Pauw Hall System (MMR Technologies, Inc) using a magnetic field of 3000 G. Square or 
rectangular thin-plate samples are mounted using silicone heat sink onto a cold stage in a sealed sample chamber connected to a vacuum pump. Indium/gallium alloy ohmic contacts are soldered at the corners of the upper surface, with triangle shapes of about $1 \mathrm{~mm}$ size. For small samples, the contact-size effect on Hall voltage measurement is corrected.

Temperature-dependent Hall measurements are realized by introducing high pressure (1,500 - 1,800 psi), high-purity, nitrogen gas (Airgas, 99.998\%) into the capillary tubes beneath the cold stage on which the sample plate is mounted. The sample chamber is prepumped to high vacuum $(<10$ mtorr $)$. As the nitrogen gas expands in the capillary tubes, it absorbs heat from the cold stage and cools the sample close to the condensation point of nitrogen. The desired temperature is maintained by an MMR H-20 temperature controller which adjusts the joule heat released from a heater beneath the cold stage to balance the cooling effect from the gas flow. The highest temperature is limited by the melting point of the indium/gallium solder. This upper temperature limit was approximately $360-400{ }^{\circ} \mathrm{C}$ in this work. The lowest temperature was often set by the signal level, and was thus limited by the sample conductivity. For samples with very low conductivity, e.g., resistivity $(\rho)$ greater than $10^{5} \mathrm{ohm}-\mathrm{cm}$, to get a reasonable Hall voltage measurement, the current had to be very small ( $<10$ nano-ampere). The experimental fluctuations of such a small current led to a high uncertainty of free carrier concentration and mobility measurements. On the other hand, for samples that are too conductive and having very small resistivities, e.g. $\rho<10^{-3}$ ohm-cm, the Hall coefficient $R_{H}=\rho \mu$ is close to zero. These measurements were also difficult to perform at lower temperatures due to the fluctuation of the Hall voltage measurements. If $\mathrm{R}_{\mathrm{H}}$ was too small, it could change sign and give a wrong message on the type of majority carriers, e.g, an n-type sample might be interpreted by the Hall measurement software as being p-type. 


\subsection{Absorption}

UV-visible-near-IR absorption experiments were carried out using a Cary-14 spectrometer system. The UV source is a deuterium lamp and the visible-NIR source is a tungsten lamp. The UV-visible detector (PMT) works reliably from 200 to $870 \mathrm{~nm}$. The PbS infrared detector works from 870 to $2600 \mathrm{~nm}$. When data were taken in the near-IR, the spectrometer path was purged using dry air in order to reduce absorptions due to water vapor.

Infrared absorption experiments were carried out with a Fourier-transform-infrared (FTIR) spectrometer (Thermo Nicolet, Nexus 870). In this system, the light source is selected to be either a white light source (QTH lamp) or a "gray-body" infrared source. The beam-splitter was chosen from $\mathrm{CaF}_{2}, \mathrm{KBr}$, or quartz depending on the scan range selected. The detector can be chosen from a mercury-cadmium-telluride detector (MCT, liquid nitrogen cooled), or a deuterated triglycine sulfate detector with a $\mathrm{KBr}$ window (DTGS-KBr). Polarized absorption measurements were performed using a wire-grid ZnSe polarizer (Molectron, Model IGP). These different source-beamsplitter-detector combinations allow FTIR absorption spectra to be recorded using a commercial OMNIC software program from 11,700 to $350 \mathrm{~cm}^{-1}$ (i.e., $855 \mathrm{~nm}$ to $28 \mu$ ).

Low temperature or temperature dependent FTIR experiments were performed in this system by placing the sample inside a continuous-flow optical cryostat (Oxford Instruments) connected to a liquid helium supply. The optical cryostat is placed in the optical path of the light beam. The desired temperature is obtained and maintained by controlling the He flow through an Intelligent Temperature Controller (Oxford ITC-502) and an Oxford Flow pump operated by an Oxford VC-41 gas flow controller. 
Both the Cary-14 and the FTIR spectra are normally acquired as absorption data. The measured signal is in the form of absorbance, given in units of optical density (OD). Optical density is the common logarithm of the ratio of incident intensity $\mathrm{I}_{0}$ to transmitted intensity $\mathrm{I}$.

$$
\text { Abs. }(\mathrm{OD})=\log _{10}\left(\mathrm{I}_{0}(\lambda) / \mathrm{I}(\lambda)\right)=\log _{10}(1 / / \mathrm{T}(\lambda))
$$

Equation 3.1 assumes the transmittance is given by $\mathrm{T}(\lambda)=\mathrm{I}(\lambda) / \mathrm{I}_{0}(\lambda)$ and ignores reflective losses at the incident and exit sample surfaces. If the reflectance (R) is included in the analysis, then the acquired data must be corrected using $\mathrm{I}(\lambda)=(1-R)^{2} \mathrm{I}_{0}(\lambda) \mathrm{T}$. The measured $\mathrm{OD}$ is larger than the actual absorbance by an amount of $\Delta \mathrm{OD}=\log _{10}(1-\mathrm{R})^{-2}$. In the transparent range of $\mathrm{ZnO}$, the refractive index $\mathrm{n} \approx 2$, hence the reflectance at normal incidence is about $\mathrm{R}=(\mathrm{n}-1)^{2} /(\mathrm{n}+1)^{2} \approx 1 / 9$. The contribution of OD due to the reflective loss is $\Delta \mathrm{OD} \approx 0.1$.

After the reflective loss correction, the absorbance (OD) value is related to the absorption coefficient $\alpha$ (in $\mathrm{cm}^{-1}$ ) and sample thickness $\mathrm{t}$ (in $\mathrm{cm}$ ) as

$$
\mathrm{I}(\lambda)=\mathrm{I}_{0}(\lambda) \exp (-\alpha \mathrm{t})
$$

From Equations 3.1 and 3.2, the absorption coefficient is obtained.

$$
\alpha(\lambda)=\mathrm{OD} /\left(\mathrm{t} \log _{10} \mathrm{e}\right)=2.303 \mathrm{OD} / \mathrm{t}\left(\mathrm{in} \mathrm{cm}^{-1}\right)
$$




\section{Chapter 4 Photoluminescence from As-grown and Gallium-Doped $\mathrm{ZnO}$}

High n-type $\mathrm{ZnO}: \mathrm{Ga}$ has been demonstrated to be an ultra-fast semiconductor scintillator for more than 50 years (Lehmann, 1966). The sub-nanosecond decay time

(Wilkinson, 2004) and medium density $\left(\rho=5.61 \mathrm{~g} / \mathrm{cm}^{-3}\right)$ make $\mathrm{ZnO}$ :Ga compare favorably to some of the best scintillators such as $\mathrm{NaI}: \mathrm{Tl}, \mathrm{CsI}: \mathrm{Tl}$, and $\mathrm{Bi}_{4} \mathrm{Ge}_{3} \mathrm{O}_{12}(\mathrm{BGO})$, while having moderate luminosity (Derenzo, 2002). Progress has been made in synthesis methods and post-synthesis treatments that result in bright $\mathrm{ZnO}: \mathrm{Ga}$ samples, especially in heavily doped powder samples used as screen materials in alpha-paticle detectors. However, knowledge on the recombination mechanisms related to the fast $\mathrm{UV}$ edge-emission in $\mathrm{ZnO}: \mathrm{Ga}$ has not been well established. Due to the large $60-\mathrm{meV}$ exciton binding energy $(60 \mathrm{meV})$ in $\mathrm{ZnO}$, freeexciton recombination (FX) and its phonon replicas are expected to survive at RT or even higher temperatures. However, in the case of heavily-doped $\mathrm{ZnO}$ :Ga for scintillator applications, recombination models such as band-band $(\mathrm{e}, \mathrm{h})$, donor-hole $\left(\mathrm{D}^{0}, \mathrm{~h}\right)$, electronacceptor $\left(\mathrm{e}, \mathrm{A}^{0}\right)$ (Derenzo, 2003), and DAP recombination models have been invoked to explain the RT PL spectra.

\subsection{Room Temperature Photoluminescence: Recombination Mechanisms}

In this section, a heavily-doped $\mathrm{ZnO}$ :Ga single crystal was studied in comparison with an as-grown undoped bulk sample. The samples were grown using high pressure melt (HPM) technique at Cermet, Inc.(Atlanta, GA), as described in Chapter 1. For the ZnO:Ga sample, the gallium dopant was directly added to the $\mathrm{ZnO}$ powder charge. The $\mathrm{ZnO}: \mathrm{Ga}$ sample has a 
light blue color, the origin of which will be discussed in Chapter 6. The undoped sample used for comparison is transparent to visible light and has a clear color.

The heavily doped Cermet $\mathrm{ZnO}$ :Ga sample has a very bright edge emission peak at 3.277 eV (378.2 nm) at RT. The undoped Cermet $\mathrm{ZnO}$ sample has an edge emission peak at $3.285 \mathrm{eV}(377.3 \mathrm{~nm})$. A comparison of the RT PL edge emissions from these two samples is shown in Figure 4.1. The PL intensities are normalized for a better comparison of peak positions and line shapes. The peak intensity of the $\mathrm{ZnO}: \mathrm{Ga}$ sample is 3.4 time brighter than that of the undoped sample under the same excitation power density. The integrated intensity from $350-420 \mathrm{~nm}(2.95-3.54 \mathrm{eV})$ for the former is 4.3 times larger than that for the latter.

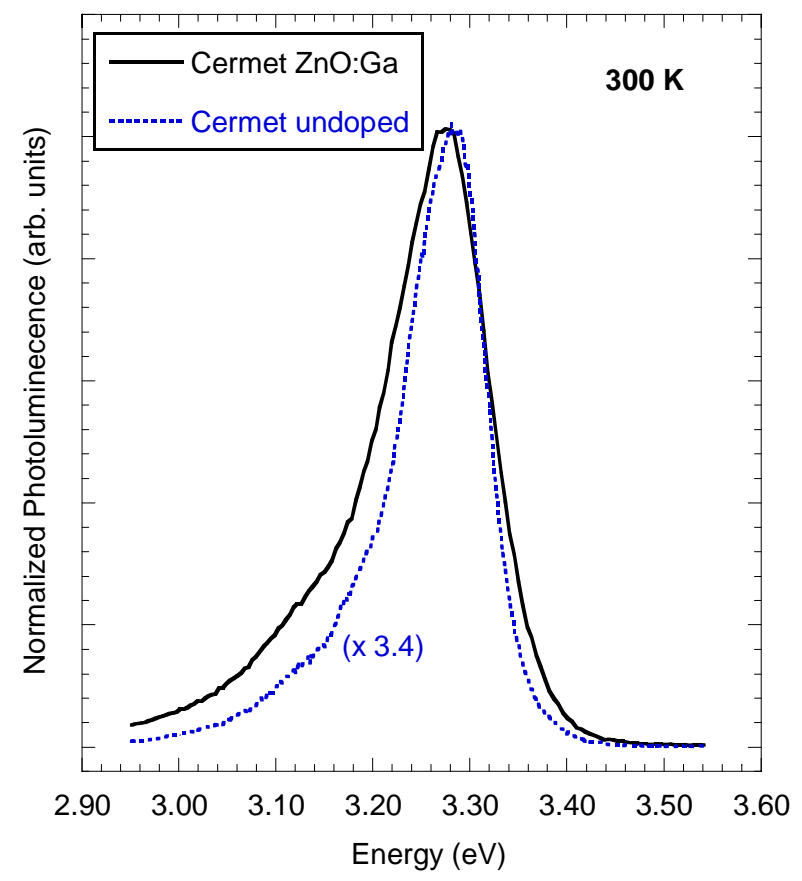

Figure 4.1 Room temperature PL from undoped and Ga-doped Cermet singlecrystal $\mathrm{ZnO}$, using $325 \mathrm{~nm}$ excitation from a continuous-wave He-Cd laser. Notice the intensities are normalized for comparison of peak positions and shapes. At $300 \mathrm{~K}$, the integrated intensity for the $\mathrm{ZnO}$ :Ga sample is 4.3 times larger than that for the undoped sample under the same excitation power density.

As shown in Fig. 4.1, although the peak energies are close for these two samples, the line-shapes are different. The PL emission for the undoped $\mathrm{ZnO}$ sample has a much steeper high-energy side, and the full-width-at-half-maximum (FWHM) is $96 \mathrm{meV}$. The ZnO:Ga sample has a FWHM of $127 \mathrm{meV}$. These two samples were also studied using Hall effect 
measurements. The undoped sample has a free carrier concentration $\mathrm{n}=6.9 \times 10^{16} \mathrm{~cm}^{-3}$ at $300 \mathrm{~K}$, which is typical for an as-grown $\mathrm{ZnO}$ single crystal without intentional doping. Previous studies on both bulk and powder samples have shown that in samples with $\mathrm{n}$ below $1 \times 10^{18} \mathrm{~cm}^{-3}$, the RT PL emission is either from free exciton (FX) recombination or from the first phonon-assisted FX recombination (FX-1LO) (Giles, 2006).

On the other hand, the $\mathrm{ZnO}: \mathrm{Ga}$ sample had $\mathrm{n}=2 \times 10^{19} \mathrm{~cm}^{-3}$ at $\mathrm{RT}$, which is close to the predicted Mott density for $\mathrm{ZnO}$. As has been discussed in Chapter 2, the bandgap renormalization due to free carriers will have a significant effect on this sample. With the band-tailing and exchange interaction effects taken into account, the electronic bandgap is expected to be around 3.21-3.22 eV at RT using Eq. 2.20. The observed RT PL peak energy is about $60-70 \mathrm{meV}$ higher than the expected band-gap, hence is not likely related to FX recombination. A possible explanation for this PL peak is the direct band-to-band (e,h) recombination, which means momentum is conserved ( $\mathrm{k}$-selection rule is valid), or indirect band-to-band (e,h) recombination, which means momentum is not conserved (k-selection rule is relaxed) (Jiang, 1982; Bugajski, 1985).

For the two Cermet $\mathrm{ZnO}$ samples discussed above, the undoped sample has a RT free carrier concentration $\mathrm{n}=6.9 \times 10^{16} \mathrm{~cm}^{-3}$. Using Eqs. $2.21-23, E_{c}{ }^{c}=2.3 \mathrm{meV}$ and $E_{c}{ }^{e}=16.3$ meV were found. With the ideal value $\mathrm{E}_{\mathrm{g}}(0)=3.372 \mathrm{eV}$ (Giles, 2006), the electronic gap in this sample is $\mathrm{E}_{\mathrm{g}}=3.372-0.0023-0.0163=3.353 \mathrm{eV}$ at $\mathrm{RT}$ using Eq. 2.20. The corresponding FX transition energy is thus predicted to be $E_{x}=3.353-0.060=3.293 \mathrm{eV}$. Considering the FX line-shape, as discussed in Chapter 2 (Section 2.2), the FX peak position will be at $3.288 \mathrm{eV}$. This prediction is supported by the observed RT PL.. Figure 4.2(a) shows the 300-K PL spectra in comparison with the calculated electronic bandgap for the 
Cermet undoped sample. The edge PL peak energy is at $3.285 \mathrm{eV}, 68 \mathrm{meV}$ below the predicted bandgap.

For the $\mathrm{ZnO}$ :Ga sample, $\mathrm{n}=2 \times 10^{19} \mathrm{~cm}^{-3}$ at $300 \mathrm{~K}$ gives $E_{c}^{c}=61.9 \mathrm{meV}$ (then $E_{c}^{c^{*}}=$ $60.2 \mathrm{meV}$ ), and $E_{c}{ }^{e}=98.8 \mathrm{meV}$. Thus, the electronic bandgap decreases to $\mathrm{E}_{\mathrm{g}}=3.372-$ $0.060-0.099=3.213 \mathrm{eV}$. On the other hand, the band-filling effect in this sample is $E_{F}{ }^{0}=$ $93 \mathrm{meV}$, and $E_{F}{ }^{*}=92 \mathrm{meV}$. Considering the near-parabolic dispersion of the top valance band, as discussed in Chapter 2 (Section 2.2), this brings the optical bandgap to $\mathrm{E}_{\mathrm{opt}}=3.213$ $+1.53 \times 0.092=3.349 \mathrm{eV}$. Fig. 4.2(b) shows the comparison of observed 300-K PL from this heavily doped $\mathrm{ZnO}$ :Ga sample and the calculated bandgap. The observed PL peak energy $(3.277 \mathrm{eV})$ falls between the electronic and optical gaps.

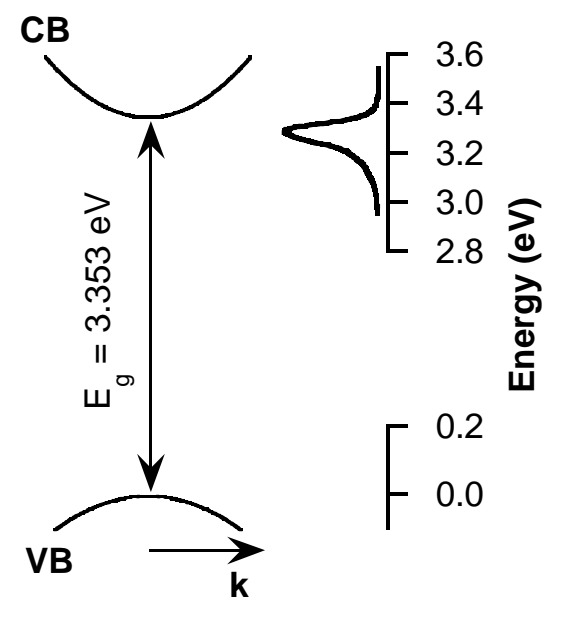

(a)

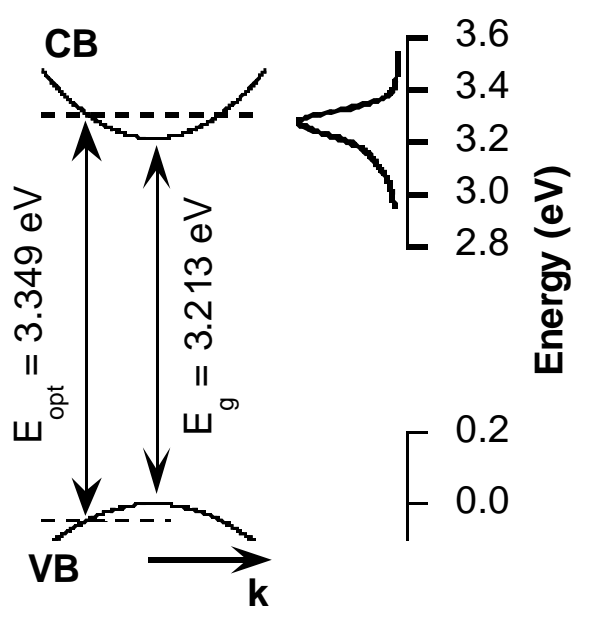

(b)

Figure 4.2. Schematic sketches of 300-K PL spectra from single-crystal samples (a) Cermet undoped $\mathrm{ZnO}$, and (b) Cermet $\mathrm{ZnO}: \mathrm{Ga}$, compared with the predicted energy gaps.

A line-shape analysis of the room temperature (RT) PL from the Cermet $\mathrm{ZnO}$ sample including FX(A), FX(B), and FX(A)-1LO phonon transitions is shown in Figure 4.3, using line shape functions of FX line and FX-1LO phonon line discussed in Chapter 2. Fitting 
parameters used here are $\mathrm{E}_{\mathrm{x}}(\mathrm{A})=3.297 \mathrm{eV}, \mathrm{E}_{\mathrm{x}}(\mathrm{B})=3.312 \mathrm{eV}$, and $\sigma=0.015 \mathrm{eV}$. The difference between the fitted value $E_{x}(A)$ and the predicted value for $E_{x}(3.293 \mathrm{eV})$ is within the experimental error. The 300-K PL was collected using the HR640/PMT system as described in Chapter 3, with a slit width of 375 microns for both entrance and exit slits.

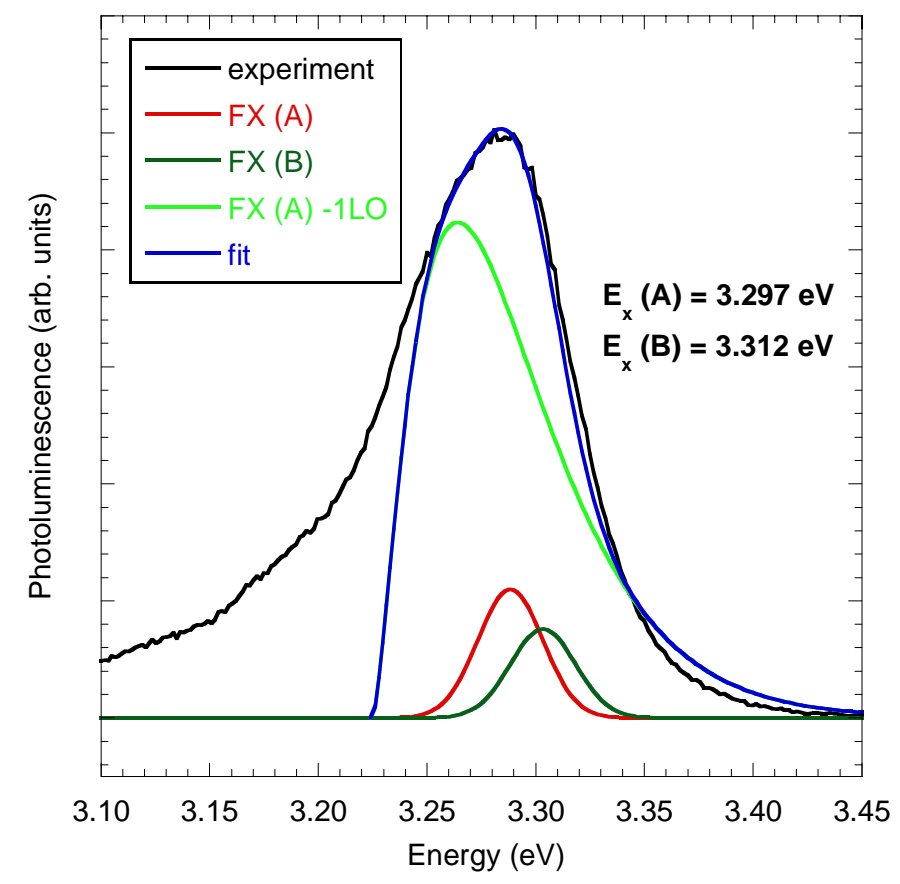

Figure 4.3. The $300-\mathrm{K}$ PL data (black) from the Cermet undoped $\mathrm{ZnO}$ sample and a theoretical fit (red) including both FX(A) (blue) and $\mathrm{FX}(\mathrm{B})$ (dark green) and the first phonon replica (FX(A)-1LO, green), as described by Eqs. 2.11-12.

Under these conditions, the system has a $5 \AA$ band pass, which gives an energy uncertainty of $\pm 5 \mathrm{meV}$ for the edge emission region. As discussed in Chapter 2, if FWHM varies as $\sim \mathrm{k}_{\mathrm{B}} \mathrm{T}$ (Bebb, 1972), it is expected to be $\approx 26 \mathrm{meV}$ at $300 \mathrm{~K}$. In the literature, an analysis of $\mathrm{ZnO}$ PL spectra including FX(A) and its first and second phonon replicas using Lorentzian lineshapes found that the homogeneous broadening of the FX emission reached a band width of $40 \mathrm{meV}$ at RT (Hauschild, 2006). The fitting shown in Fig. 4.3 with $\sigma=0.015 \mathrm{eV}$ gives a FWHM value of $35 \mathrm{meV}$ at RT for free excitons, which is smaller than the reported value because I included FX(B) to fit the higher energy side of the FX emission peak. The inclusion of $\mathrm{FX}(\mathrm{B})$ is supported by the temperature dependent PL, which will be discussed in the next section. For photon energy higher than $3.35 \mathrm{eV}$, the PL spectrum is lower than the 
fitting, which can be explained by self-absorption. The lower energy side of the peak is mainly due to the FX-mLO $(\mathrm{m}>1)$ transitions and were not included in the fitting.

As shown in Fig. 4.2(b), the peak of the PL band from the Cermet ZnO:Ga falls between the predicted electronic bandgap $\left(\mathrm{E}_{\mathrm{g}}\right)$ and the optical bandgap $\left(\mathrm{E}_{\mathrm{opt}}\right)$. To assign the recombination of the main peak needs understanding of the line shape of the PL peak.

Considering the high $\mathrm{n}$ value obtained from the Hall measurement, an indirect band-to-band transition process was first invoked to fit the main peak (Jiang, 1982):

$$
\begin{aligned}
& F(E) \propto \int_{0}^{E-E_{g}} d E^{\prime} E^{\prime^{1 / 2}}\left(E-E_{g}-E^{\prime}\right)^{1 / 2}\left[1+\exp \left(\frac{E^{\prime}-F_{c}^{\prime}}{k_{B} T}\right)\right]^{-1} \\
& \times\left\{1-\left[1+\exp \left(-\frac{E+E_{g}+E^{\prime}}{k_{B} T}+\frac{F_{v}^{\prime}}{k_{B} T}\right)\right]^{-1}\right\}
\end{aligned}
$$

Here $F_{c}^{\prime}$ and $F_{v}^{\prime}$ are the quasi-Fermi energies for electrons and holes, respectively. The momentum-conservation rule is relaxed in this expression, as has been used to explain the

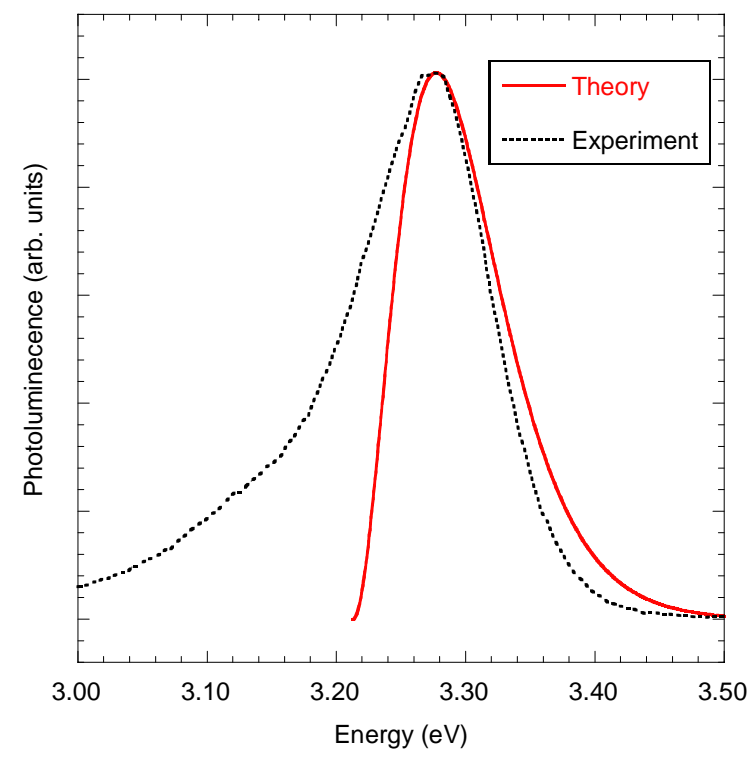

Figure 4.4. RT PL data (black, dotted line) from Cermet $\mathrm{ZnO}: \mathrm{Ga}$ and a theoretical fit (red, solid line) using the indirect transition model Eq. 4.1.

broad PL peak from heavily doped InP (Jiang, 1982). The lineshape predicted by Eq. 4.1 is shown in Fig. 4.4 (red, solid line), the peak position is predicted at $3.277 \mathrm{eV}$, using fitting 
parameters $\mathrm{E}_{\mathrm{g}}=3.213 \mathrm{eV}$, and $F_{c}^{\prime}=F_{v}^{\prime}=0$. The agreement between the transition model and the PL data (black, dotted line) is rather poor on the high-energy side.

The next model to consider is direct band-to-band $(\mathrm{e}, \mathrm{h})$ recombination which includes momentum-conservation. The line shape predicted from this model is given by

$$
F(E) \sim\left(E-E_{g}\right)^{1 / 2} \frac{1}{1+\exp \left(\frac{E-E_{g}-E_{F}}{k_{B} T}\right)}
$$

where the density-of-states for a parabolic conduction band is assumed, and the Fermi-Dirac distribution function describes the thermalized distribution of occupied states. This expression should be appropriate at low excitation intensities. The exponential function in the denominator of Eq. 4.2 is often assumed to be $>1$, and the expression is simplified as

$$
\mathrm{F}(\mathrm{E}) \propto\left(\mathrm{E}-\mathrm{E}_{\mathrm{g}}\right)^{1 / 2} \exp \left[-\left(\mathrm{E}-\mathrm{E}_{\mathrm{g}}-\mathrm{E}_{\mathrm{F}}\right) / \mathrm{k}_{\mathrm{B}} \mathrm{T}\right]
$$

which will give an abrupt low-energy cut-off at $\mathrm{E}=\mathrm{E}_{\mathrm{g}}$, and a temperature-dependent peak position according to

$$
E_{m}=E_{g}+k_{B} T / 2
$$

When Eq. 4.3 was used to fit the measured RT PL data from the Cermat $\mathrm{ZnO}: \mathrm{Ga}$ sample, it was found that the model was over-simplified in this case. While the peak position can be produced with $\mathrm{E}_{\mathrm{g}}=3.265 \mathrm{eV}$, which is about $50 \mathrm{meV}$ higher than predicted from bandgap renormalization, the narrow lineshape determined by Eq. 4.3 couldn't account for the main peak. Because of the high free-carrier density in this sample, $\mathrm{E}_{\mathrm{F}}^{0}=92 \mathrm{meV}$ was predicted using Eq. 2.24. As a consequence, the exponential term in the denominator of Eq. 4.2 is no longer far greater than unity in the PL peak range and the Fermi-Dirac distribution cannot be simplified as appearing in Eq. 4.3. In this situation, Eq. 4.2 was used to fit the RT PL peak. Although the peak position can be reproduced using the predicted values $E_{g}=3.213$ 
$\mathrm{eV}$ and $\mathrm{E}_{\mathrm{F}}=0.092 \mathrm{eV}$, the best fit for both the peak position and the higher energy side was obtained with $\mathrm{E}_{\mathrm{g}}=3.224 \mathrm{eV}$ and $\mathrm{E}_{\mathrm{F}}=0.076 \mathrm{eV}$, as shown in Fig. 4.5. The fitted $\mathrm{E}_{\mathrm{g}}$ value is $11 \mathrm{meV}$ higher than the prediction, which defines the smallest energy needed to be absorbed so as to create a free electron in the heavily filled conduction band that contributes to the (e,h) transition. The smaller value of $\mathrm{E}_{\mathrm{F}}$ may indicate the change of the $\mathrm{CB}$ curvature due to the heavy doping and merging of the donor band with the $\mathrm{CB}$. If the $\mathrm{CB}$ can still be described as parabolic, a larger effective mass is needed when using Eq. 2.24.

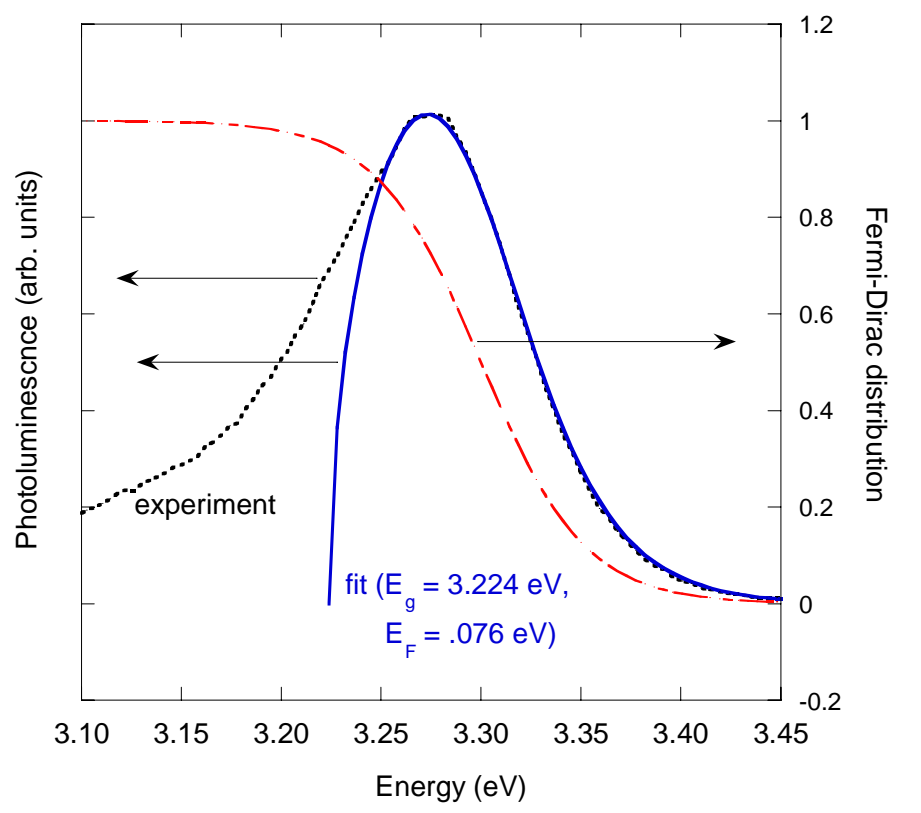

Figure 4.5. 300-K PL data (dotted line) from $\mathrm{ZnO}: \mathrm{Ga}$ and a theoretical fit (solid line) using the direct transition model described in Eq. 4.2. The dash-dot line is the 300-K Fermi-Dirac distribution function, shown for reference.

The RT PL peak from the ZnO:Ga sample has a lower-energy tail extending much farther than that from the undoped sample (Fig. 4.1). The former shows an additional weak band at around $3.14 \mathrm{eV}$, although not easy to resolve from the main peak. From the bandgap rerenormalization, the electronic band-gap for this sample is $\mathrm{E}_{\mathrm{g}}=3.213 \mathrm{eV}$, giving a FX transition energy of $E_{x}=3.213-0.06=3.153 \mathrm{eV}$, and the FX PL peak is expected to be at $3.148 \mathrm{eV}$ at RT. This is close to the observed additional peak on the lower energy side tail. In order to distinguish this lower energy emission from the main peak, a selective-excitation 
PL experiment was performed using a short-arc Xe lamp (Oriel). A set of excitation wavelengths from 325 to $383 \mathrm{~nm}$ ( 3.81 to $3.24 \mathrm{eV}$ ) were used, and the effects of resonantly pumping above or below the optical band gap $\left(\mathrm{E}_{\mathrm{opt}}=3.349 \mathrm{eV}\right)$ were determined. Figure 4.6 shows the representative selective-excitation PL spectra. For excitation wavelength longer than $370 \mathrm{~nm}(3.35 \mathrm{eV})$, the line shape doesn't change significantly for different excitation, and the (e,h) emission is the major feature. As the excitation wavelength further increases (i.e., photon energy $<3.35 \mathrm{eV}$ ), the band around $3.14 \mathrm{eV}$ becomes clearly resolvable, and is the strongest at the lowest excitation energy, $3.24 \mathrm{eV}(383 \mathrm{~nm})$. These results suggest that

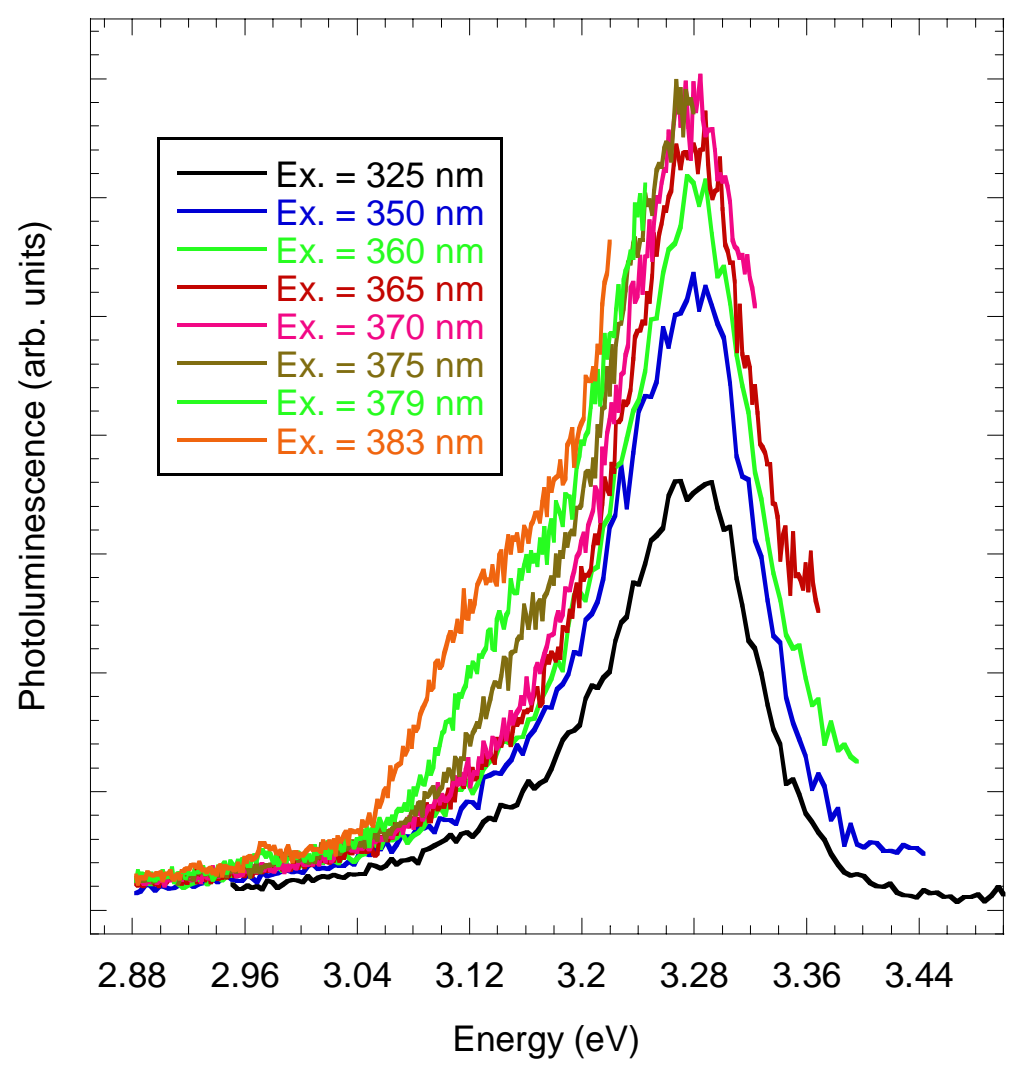

Figure 4.6 RT PL from Cermet $\mathrm{ZnO}$ :Ga sample using selected excitations from a short-arc Xe lamp, with wavelength ranging from $325-383 \mathrm{~nm}$.

the peak around $3.14 \mathrm{eV}$ has a different origin than the $3.28 \mathrm{eV}(\mathrm{e}, \mathrm{h})$ transition. Based on the prediction of $\mathrm{E}_{\mathrm{g}}$ and $\mathrm{E}_{\mathrm{x}}, \mathrm{I}$ assign it to the $\mathrm{FX}$ emission. This assignment implies that either FX can still form at such heavy doping $\left(\mathrm{n}=2 \times 10^{19} \mathrm{~cm}^{-3}\right.$ is close to the Mott density), or the sample has two different phases: one with $\mathrm{n}=2 \times 10^{19} \mathrm{~cm}^{-3}$, the other with much 
lower $\mathrm{n}$ such that FX emission is still the major recombination at RT. Below-bandgap excitation using laser (e.g., $364 \mathrm{~nm}$ ) may help eventually determine the nature of this band.

\subsection{Temperature-dependent Photoluminescence}

As sample temperature decreases below RT, the emission bands from different PL recombinations such as FX and its LO-phonon replicas become narrower in linewidth due to a reduction in thermal broadening. These lines also become more intense at lower temperature due to a reduction in competing non-radiative recombinations. Although $\mathrm{ZnO}$ light emitters will operate primarily at room temperature, the temperature dependences of the different recombination mechanisms can often help identify the particular recombination paths. In this section, the liquid-He-temperature PL spectra from the undoped and heavily doped Cermet samples discussed in Section 4.1 are first shown. Heavy donor doping produces dramatic changes in the 5-K PL spectra from bulk single crystals. Then, the temperature-dependent PL spectra from the two samples will be presented. A comparison of the temperature dependences of PL and free carrier concentrations allows insight into the temperature dependence of the $\mathrm{ZnO}$ instrinsic bandgap.

The 5-K PL spectra from the two Cermet samples are shown in Figures 4.7. and 4.8. For the undoped sample, the dominant PL emissions at $5 \mathrm{~K}$ are the donor-bound exciton $\left(\mathrm{D}^{0} \mathrm{X}\right)$ lines near $3.36 \mathrm{eV}$, which are assigned to substitutional donors, most likely $\mathrm{Al}$ and Ga. The first, second, and third LO-phonon replicas of the dominant $\mathrm{D}^{0} \mathrm{X}$ line could be clearly seen at lower energies with energy separations of about $72 \mathrm{meV}$ (see top stick diagram). Another sharp $\mathrm{D}^{0} \mathrm{X}$ line at $3.357 \mathrm{eV}$ is associated with substitutional In. As shown in an expanded view in Fig. 4.8, on the higher energy side, the first excited state of the donor bound exciton $\left(D^{0} X, n=2\right)$ is observed at $3.373 \mathrm{eV}$. The energy difference between the $\mathrm{D}^{0} \mathrm{X}$ 
line and its first excited state line is $\Delta \mathrm{E}=3.373-3.3605=0.0125 \mathrm{eV}$, and the binding energy of $D^{0} X$ is $E_{B}=(4 / 3)(12.5)=16.7 \mathrm{meV}$. The free exciton transition energy $E_{x}$

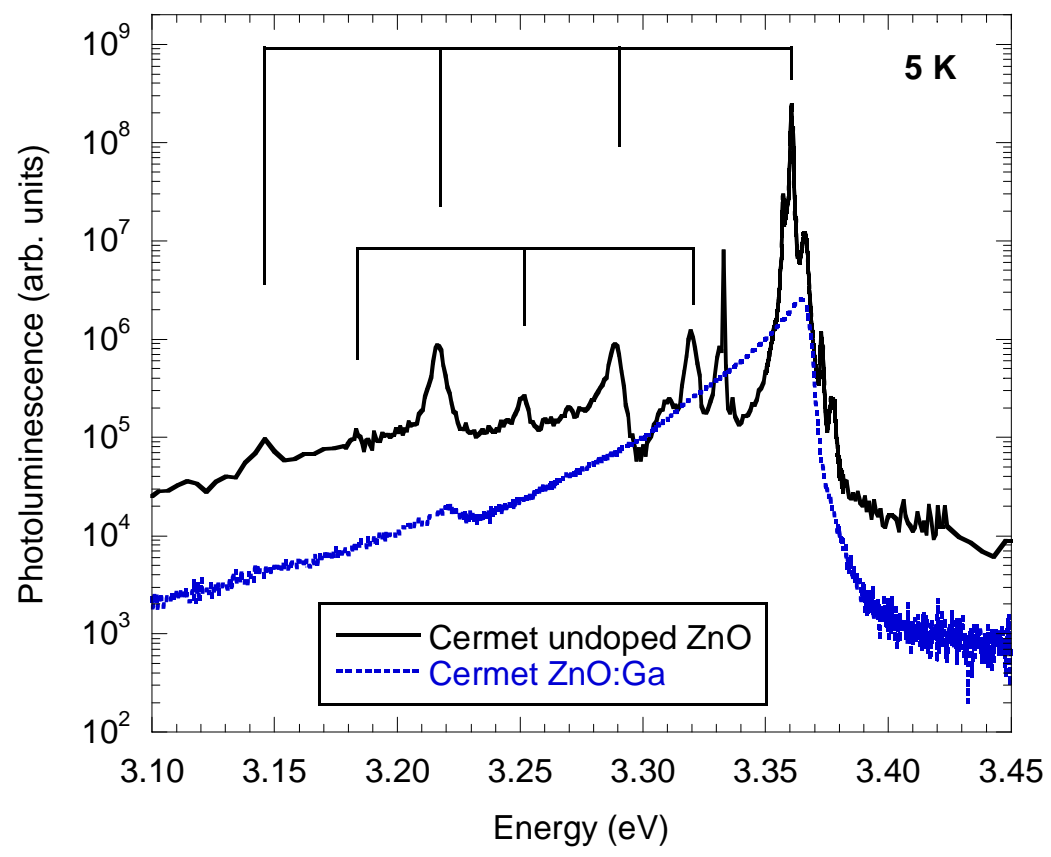

Figure 4.7. 5-K PL from Cermet undoped $\mathrm{ZnO}$ (solid line) and Cermet $\mathrm{ZnO}: \mathrm{Ga}$ (dotted line). The stick lines show the cascade of phonon replicas separated by the LO phonon energy ( 72 meV). An expanded view is shown in Fig. 4.8.

Notice the logarithmic vertical scale.

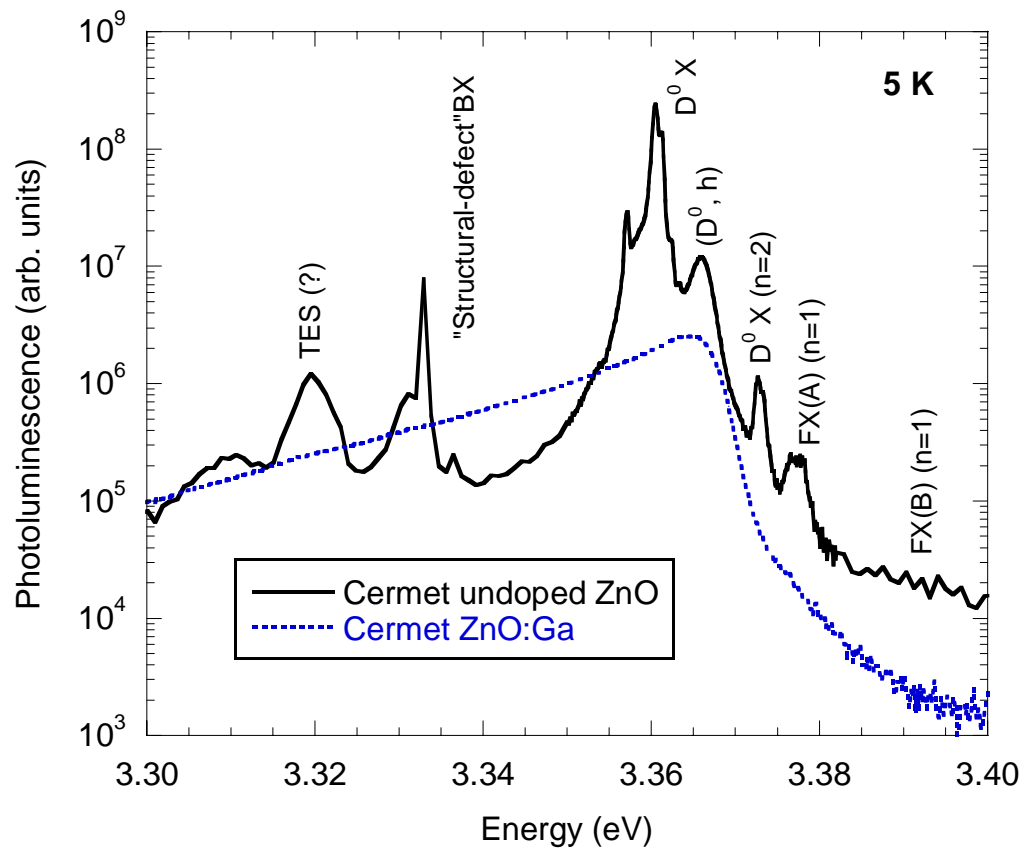

Figure 4.8. An expanded view of Fig. 4.7 for a better comparison of the nearband-edge emissions. Notice the logarithmic vertical scale.

can be determined by $\mathrm{E}_{\mathrm{x}}=3.3605+0.0167 \approx 3.377 \mathrm{eV}$, which is equal to the ground state energy of FX (A) in Fig. 4.8, and gives a bandgap $\mathrm{E}_{\mathrm{g}}(\mathrm{A})=3.437 \mathrm{eV}$. The second excited 
state of the $\mathrm{D}^{0} \mathrm{X}$ transition $\left(\mathrm{D}^{0} \mathrm{X}, \mathrm{n}=3\right.$ ) has an energy of $\left.3.377-0.0167 / 9\right)=3.375 \mathrm{eV}$, and merges with the FX(A) line. The FX(B) band locates at about $15 \mathrm{meV}$ higher than the $\mathrm{FX}(\mathrm{A})$. The relatively broad peak at $3.366 \mathrm{eV}$ is most likely due to a $\left(\mathrm{D}^{0}, \mathrm{~h}\right)$ transition, corresponding to a shallow donor with an ionization energy of $\mathrm{E}_{\mathrm{D}}=3.437-3.366=0.071 \mathrm{eV}\left(\mathrm{E}_{\mathrm{D}}=72 \mathrm{meV}\right.$ was obtained from Hall data analysis on this sample, as will be discussed in Chapter 5). Compared to the donor energy, the Haynes factor is determined as $\alpha=16.7 / 71 \approx 0.235$ for this sample. On the lower energy side, the origin of the sharp line at $3.332 \mathrm{eV}$ is not clear, although one group has concluded that this is an excitonic line bound to a structural defect (Meyer, 2004). The same group assigned the broad line at $3.3195 \mathrm{eV}$ and its LO-phonon replicas as two-electron satellite (TES) lines of the dominant $\mathrm{D}^{0} \mathrm{X}$ line. However, as will be discussed below, there is possibly a different explanation for this emission.

For the heavily doped $\mathrm{ZnO}$ :Ga sample, the 5-K PL exhibits only a broad emission band peaking near $3.364 \mathrm{eV}$. Compared to PL data taken at much higher temperatures, besides the direct $(\mathrm{e}, \mathrm{h})$ transition, the $5-\mathrm{K}$ spectrum from $\mathrm{ZnO}$ :Ga may also contain contributions from $\mathrm{FX}, \mathrm{D}^{0} \mathrm{X}$ and $\left(\mathrm{D}^{0}, \mathrm{~h}\right)$. Due to heavy doping, the tail of the $\mathrm{CB}$ minimum becomes a mixture of states, and these separate contributions cannot be easily distinguished.

Figures 4.9-10 show the temperature-dependent PL from the undoped Cermet $\mathrm{ZnO}$ from 5 to $300 \mathrm{~K}$. As sample temperature increases, the quenching behavior of free exciton, bound exciton and other recombination processes are very different. A bound exciton will first dissociate into a free exciton and then from the free exciton into a free hole and a free electron. This two-step dissociation can be described as (Yu, 2001)

$$
\mathrm{I}(\mathrm{T})=\mathrm{I}(0) /\left[1+\mathrm{C}_{1} \exp \left(-\mathrm{E}_{\mathrm{A} 1} / \mathrm{k}_{\mathrm{B}} \mathrm{T}\right)+\mathrm{C}_{2} \exp \left(-\mathrm{E}_{\mathrm{A} 2} / \mathrm{k}_{\mathrm{B}} \mathrm{T}\right)\right]
$$

where the first activation energy $\mathrm{E}_{\mathrm{A} 1}$ is close to the binding energy of the bound exciton, and 
the second activation energy $E_{A 2}$ is expected to be close to the FX binding energy $\left(R_{\mathrm{x}}\right)$ or donor ionization energy $\left(E_{D}\right)$. As sample temperature increases, the first step is the major

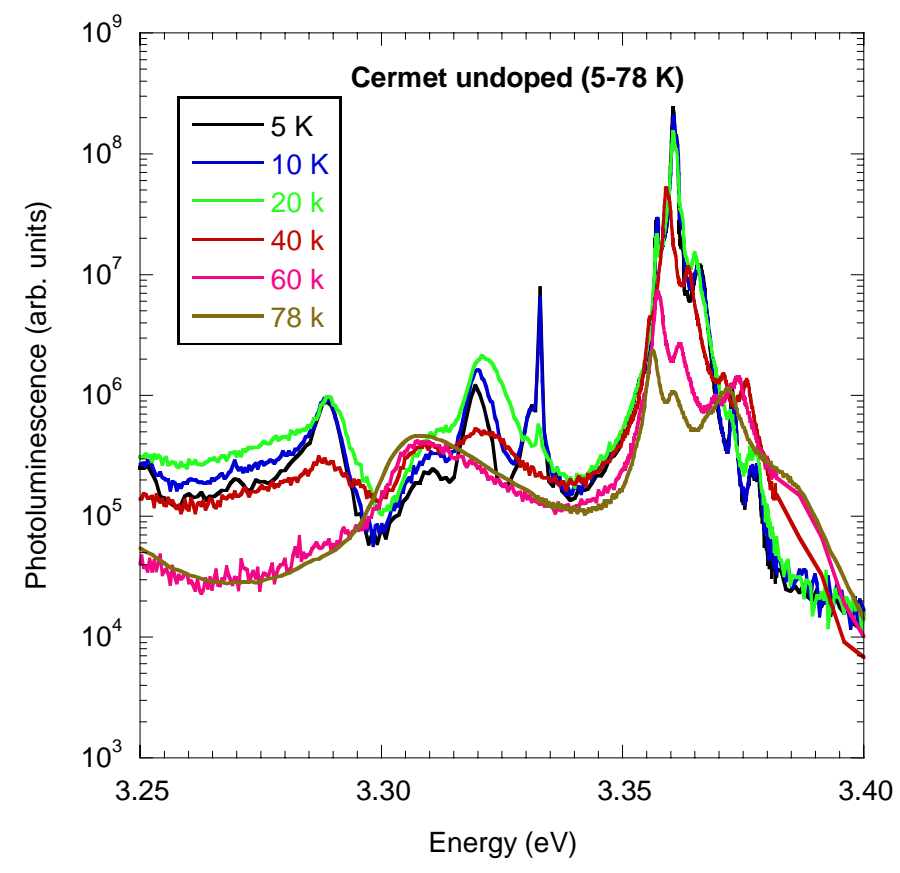

Figure 4.9. Temperature-dependent PL from Cermet undoped $\mathrm{ZnO}$ from 5 to $78 \mathrm{~K}$. Notice the logarithmic vertical scale.

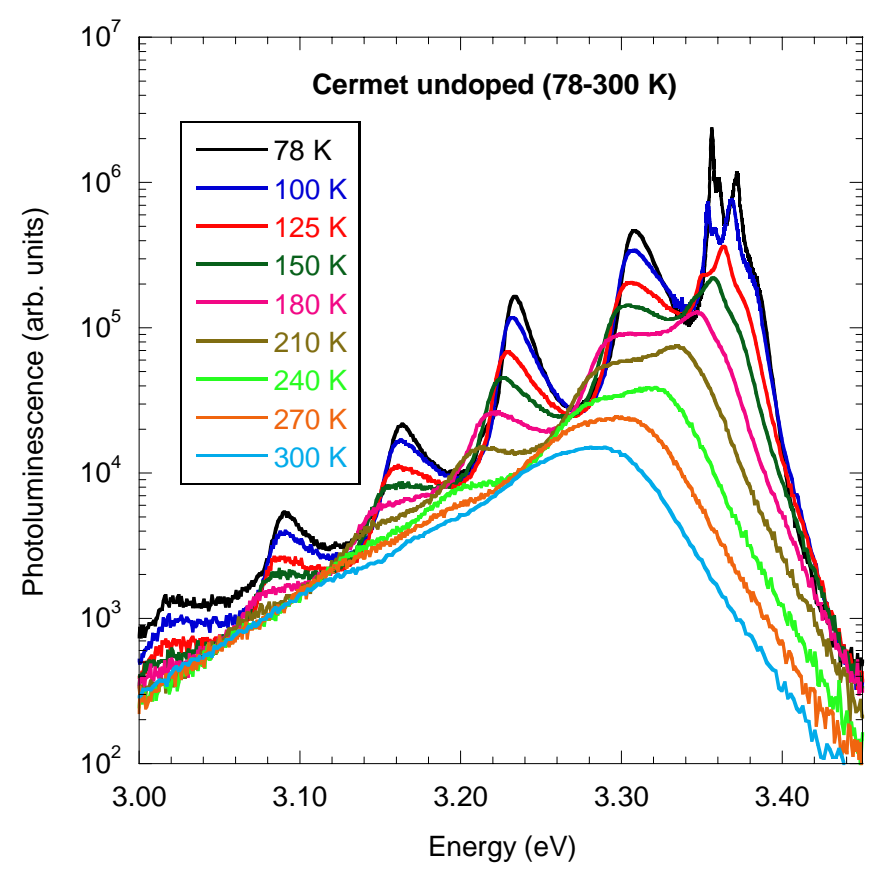

Figure 4.10. Temperature-dependent PL from Cermet undoped $\mathrm{ZnO}$ from 78 to $300 \mathrm{~K}$. The energy range is three times larger than Fig. 4.9. Notice the logarithmic vertical scale.

process affecting the PL intensity and the bound exciton lines quench very fast. The intensity of the major $\mathrm{D}^{0} \mathrm{X}$ emission decreases by two orders of magnitude as temperature goes from 5 
$\mathrm{K}$ to $78 \mathrm{~K}$. The $\mathrm{FX}(\mathrm{A})$ intensity increases relative to the $\mathrm{D}^{0} \mathrm{X}$ bands for this temperature range, aided by the first-step dissociation of $\mathrm{D}^{0} \mathrm{X}$. At $78 \mathrm{~K}$, the $\mathrm{FX}(\mathrm{A})$ intensity is comparable to $\mathrm{D}^{0} \mathrm{X}$, and its LO-phonon replicas become the main feature in the energy range of 3.0-3.35 eV, with the $5^{\text {th }}$ replica still clearly recognizable (Fig. 4.10). It is interesting to notice that for temperature higher than $60 \mathrm{~K}$, as the FX(A) band becomes stronger compared to the $\mathrm{D}^{0} \mathrm{X}$ band, the $\mathrm{FX}(\mathrm{B})$ band becomes stronger too, adding a shoulder band to the higher energy side of FX(A), and is recognizable until $180 \mathrm{~K}$. This is supportive to the inclusion of FX(B) contribution in the 300-K lineshape analysis in section 4.1. The sharp "structuredefect" bound exciton line disappears when temperature goes higher than $20 \mathrm{~K}$. This quenching process is even faster than that of the dominant $\mathrm{D}^{0} \mathrm{X}$ lines, and may indicate that the dissociation of this line has a different pattern from that described by Eq. 4.5.

As discussed above, the broad peak at $3.3195 \mathrm{eV}$ at $5 \mathrm{~K}$ has been attributed to the TES line of the donor-bound exciton (Meyer, 2004). Such an assignment means that a TES line appears at $\Delta \mathrm{E}=(3 / 4) \mathrm{E}_{\mathrm{D}}$ below the $\mathrm{D}^{0} \mathrm{X}$ line (see Ch. 2), where $\mathrm{E}_{\mathrm{D}}$ is the ionization energy of the associated donor. The separation of this line and the major $\mathrm{D}^{0} \mathrm{X}$ line (Fig. 4.8) is $\Delta \mathrm{E}=$ 3.3605-3.3195 $=0.041 \mathrm{eV}$, then $\mathrm{E}_{\mathrm{D}}=0.055$ can be drawn. This implies that $\mathrm{a}\left(\mathrm{D}^{0}, \mathrm{~h}\right)$ may appear at about $5 \mathrm{meV}$ higher than the FX(A) line, which is not seen in this sample. Further, using the $\mathrm{D}^{0} \mathrm{X}$ binding energy $(16.7 \mathrm{eV})$ and this $\mathrm{E}_{\mathrm{D}}$ value, the Haynes factor will be $16.7 / 55$ $=0.3$, much lager than the well known value $(0.1-0.2)$ for $\mathrm{D}^{0} \mathrm{X}$ (Haynes, 1960). The assignment of the "TES" line also suggests that it stays at a fixed distance in energy from the associated $\mathrm{D}^{0} \mathrm{X}$, assuming that the donor ionization energy is independent of temperature. For the Cermet undoped sample, as shown in Fig. 4.8, the peak energy of the main $\mathrm{D}^{0} \mathrm{X}$ doesn't change ( $3.3605 \mathrm{eV}$ ) from $5 \mathrm{~K}$ to $20 \mathrm{~K}$, and drops to $3.3591 \mathrm{eV}$ at $40 \mathrm{~K}$ before further 
decreasing as T increases. The "TES" line observed here does not follow this pattern. The peak energy of this line increases from $3.3195 \mathrm{eV}(5 \mathrm{~K})$ to $3.3199 \mathrm{eV}(10 \mathrm{~K})$ to $3.3211 \mathrm{eV}$ $(20 \mathrm{~K})$, then decreases to $3.3208 \mathrm{eV}$ at $40 \mathrm{~K}$. It totally disappears for $\mathrm{T} \geq 60 \mathrm{~K}$, although the $\mathrm{D}^{0} \mathrm{X}$ line will dominate till $\mathrm{T}=78 \mathrm{~K}$. A possible explanation for the behavior of this line is that, instead of a "TES" line of the $\mathrm{D}^{0} \mathrm{X}$ transition, it could be associated with a free-electron to neutral-acceptor transition $\left(\mathrm{e}, \mathrm{A}^{0}\right)$. The peak position of the $\left(\mathrm{e}, \mathrm{A}^{0}\right)$ transition has a temperature dependence described by Eq. 2.14. At low temperatures, the peak position will increase as $E\left(e, A^{0}\right)=E_{g}-E_{A}+(1 / 2) k_{B} T$, until the shrinkage of the band-gap surpasses $(1 / 2) \mathrm{k}_{\mathrm{B}} \mathrm{T}$. At higher temperatures, since all the acceptors are ionized and no longer trap holes on them, and the $\left(\mathrm{e}, \mathrm{A}^{0}\right)$ transition process gives way to $\mathrm{FX}$ recombinations. Attributing this line to an $\left(\mathrm{e}, \mathrm{A}^{0}\right)$ transition was also suggested by another group, where lineshape analysis of this line from CL spectra led to an acceptor binding energy of $130 \mathrm{meV}$ (Schirra, 2008).

When temperature goes above $150 \mathrm{~K}$, the $\mathrm{D}^{0} \mathrm{X}$ emission disappears and the $\mathrm{FX}$ quenching occurs. As the FX and its LO-phonon replicas show significant thermal broadening, the distance between the FX and the FX-1LO line decreases, since the peak position of the latter is given as $\mathrm{E}_{\mathrm{FX}-\mathrm{LO}}=\mathrm{E}_{\mathrm{x}}-\hbar \omega_{\mathrm{LO}}+(3 / 2) \mathrm{k}_{\mathrm{B}} \mathrm{T}$. At $300 \mathrm{~K}$, the peak energy of the FX emission is expected to be $\mathrm{E}_{\mathrm{FX}}=\mathrm{E}_{\mathrm{x}}-\mathrm{k}_{\mathrm{B}} \mathrm{T} /(8 \ln 2) \approx \mathrm{E}_{\mathrm{x}}-5 \mathrm{meV}$, and the peak of FX$1 \mathrm{LO}$ line peak is at $\mathrm{E}_{\mathrm{FX}-1 \mathrm{LO}}=\mathrm{E}_{\mathrm{x}}-72 \mathrm{meV}+(3 / 2)(26 \mathrm{meV}) \approx \mathrm{E}_{\mathrm{x}}-33 \mathrm{meV}$. For $\mathrm{T}>180 \mathrm{~K}$, the two lines merge and it's not easy to tell which is dominant unless a detailed line-shape analysis is performed. However, as could be seen in Fig. 4.9, the merged PL peak from 180$300 \mathrm{~K}$ has an almost straight line on the higher energy side. In a semi-logarithmic plot, this indicates an energy dependence of $\sim \exp \left(-\mathrm{E} / \mathrm{k}_{\mathrm{B}} \mathrm{T}\right)$. Thus, the high-T PL emission is mainly from the first LO-phonon assisted recombination, and the FX contribution is a small portion 
of the merged peak. This is similar to what has been observed from single crystals grown from Eagle-Picher samples grown by seeded chemical vapor transport (SCVT) method (Wang, 2003), but the FX emission claims the PL peak for the Cermet sample.

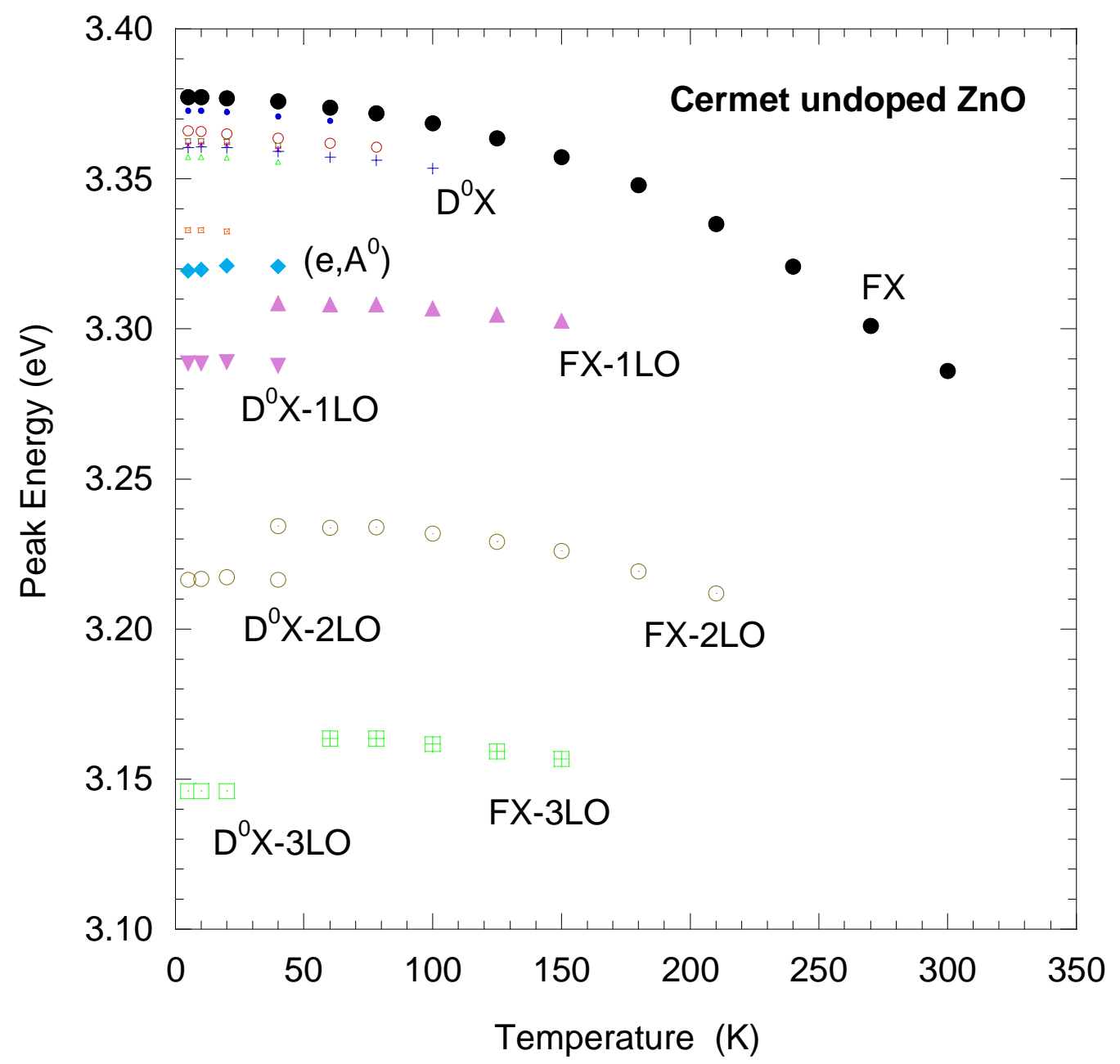

Figure 4.11. Temperature-dependent PL peak positions from Cermet undoped $\mathrm{ZnO}$ (5 - 300 $\mathrm{K})$.

The temperature-dependent PL data from the Cermet undoped $\mathrm{ZnO}$ is also presented in Figure 4.11, where the peak positions associated with different transitions are plotted versus temperature. The $\mathrm{D}^{0} \mathrm{X}$ emissions quench as temperature reaches $100 \mathrm{~K}$, and their LOphonon replicas quench at even lower temperatures. FX transitions survive to higher temperatures, and the FX-2LO can be clearly seen till $210 \mathrm{~K}$. For $\mathrm{T} \geq 180 \mathrm{~K}$, due to the 
broadening and merging of the FX phonon replicas, the exact peak energies could be obtained only by line-shape analysis. The diamonds in Fig. 4.11 represent the peak energies of the so-called "TES" line, and its T-dependence behavior makes it more likely an (e, $\left.\mathrm{A}^{0}\right)$ band instead (so it is labeled accordingly).

Figure 4.12 shows the temperature-dependent PL from the heavily doped Cermet $\mathrm{ZnO}: \mathrm{Ga}$ sample. Compared to the spectra from the undoped sample which contained many discrete lines, the PL from the $\mathrm{ZnO}$ :Ga sample is broad and contains a single featureless peak. The emission has a slower quenching rate than the undoped sample. From $5 \mathrm{~K}$ to $300 \mathrm{~K}$, the peak intensity in the doped sample decreases by a factor of only 50 . The integrated PL intensity over the edge emission region $(350-420 \mathrm{~nm})$ decreases by a factor of 8 . This is good for the scintillator application, where bright RT UV emission is desired. In contrast, the quenching for the undoped $\mathrm{ZnO}$ sample is faster. From 5 to $300 \mathrm{~K}$, the integrated edge emission for the undoped sample over the same region decreases by $\sim 250$.

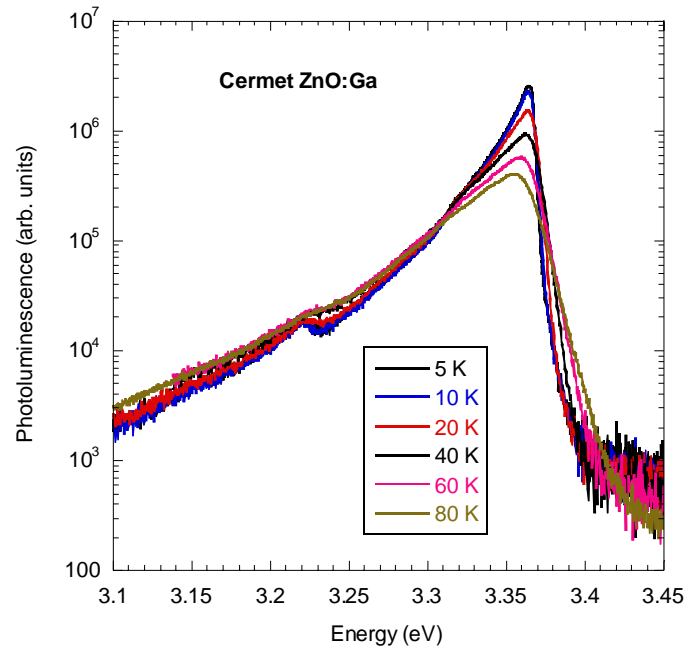

(a)

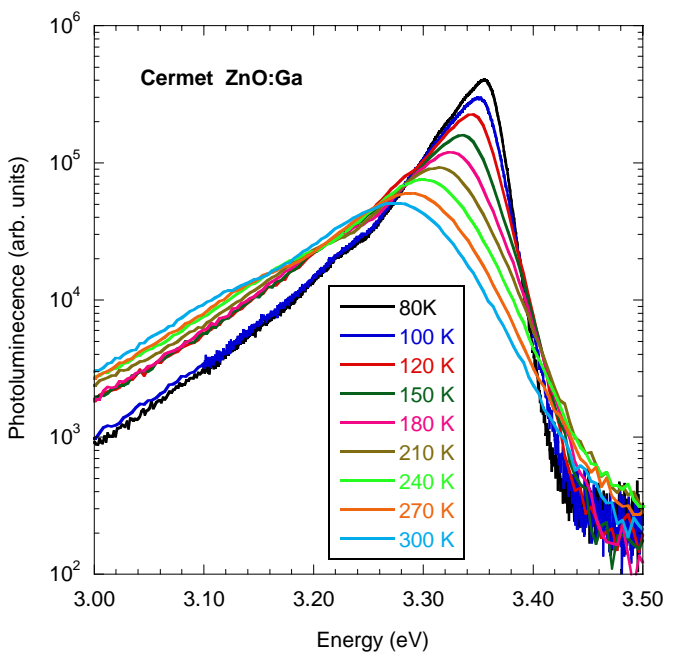

(b)

Figure 4.12. Temperature-dependent PL from Cermet ZnO:Ga. (a) 5-80 K; (b) 80-300 K. Notice the logarithmic vertical scale. 
Due to competition with non-radiative recombinations, the quenching of a radiative recombination often can be expressed quantitatively with an effective activation energy.

$$
\mathrm{I}(\mathrm{T})=\mathrm{I}(0) /\left[1+\mathrm{C} \exp \left(-\mathrm{E}_{\mathrm{A}} / \mathrm{k}_{\mathrm{B}} \mathrm{T}\right)\right]
$$

where $\mathrm{E}_{\mathrm{A}}$ is the activation energy and $\mathrm{C}=\mathrm{P}_{\mathrm{nr}} / \mathrm{P}_{\mathrm{r}}$ is the ratio of non-radiative to radiative probabilities. Figure 4.13 shows integrated PL intensity for the range of 350-420 nm for the $\mathrm{ZnO}: \mathrm{Ga}$ sample (open circles) and the undoped $\mathrm{ZnO}$ sample (open squares). Eq. 4.6 was used to fit the data from the $\mathrm{ZnO}$ :Ga sample, and an effective activation energy $\mathrm{E}_{\mathrm{A}}=13$ meV was obtained (Fig. 4.13, curve a). A two-level quenching model

$$
\mathrm{I}(\mathrm{T})=\mathrm{I}_{1}(0) /\left[1+\mathrm{C}_{1} \exp \left(-\mathrm{E}_{\mathrm{A} 1} / \mathrm{k}_{\mathrm{B}} \mathrm{T}\right)\right]+\mathrm{I}_{2}(0) /\left[1+\mathrm{C}_{2} \exp \left(-\mathrm{E}_{\mathrm{A} 2} / \mathrm{k}_{\mathrm{B}} \mathrm{T}\right)\right]
$$

was used to fit the integrated PL intensity for the undoped $\mathrm{ZnO}$ sample (Fig. 4.13, curve b), and the activation energies were found to be $\mathrm{E}_{\mathrm{A} 1}=18 \mathrm{meV}$, and $\mathrm{E}_{\mathrm{A} 2}=73 \mathrm{meV}$. The first activation energy is close to the binding energy of the DBX lines $(16.7 \mathrm{meV})$. The second activation energy is close to the donor ionization energy drawn from the $\left(D^{0}, h\right)$ peak energy at 5-K PL (71 meV), or obtained from Hall data (72 meV, as will be discussed in Chapter 5).

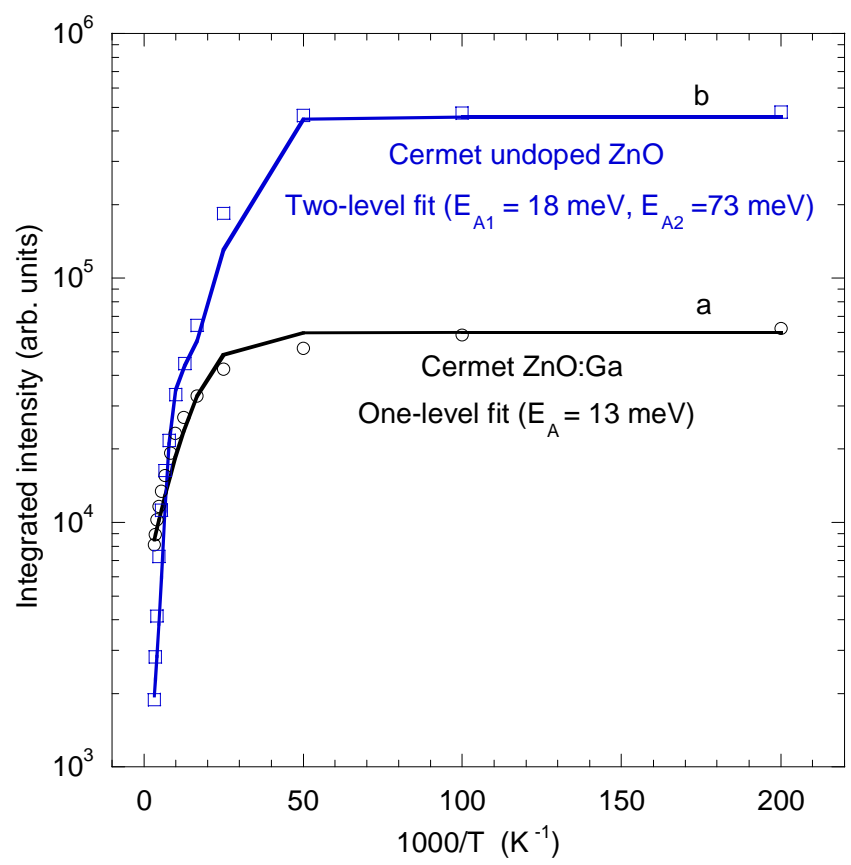

Figure 4.13. Integrated edge emission intensities $(350-420 \mathrm{~nm})$ from single crystal Cermet $\mathrm{ZnO}$ (solid circles) and Cermet $\mathrm{ZnO}: \mathrm{Ga}$ (open squares). The solid lines are theoretical fit using Eqs. (4.6-7). The activation energies from fit are indicated. Notice the logarithmic vertical scale. 
When temperature-dependent Hall data and PL data are both available, as in the case of the Cermet undoped sample in this study, they can be used to analyze the temperaturedependent behavior of the bandgap. The approach in this work is unique since it will also include the effects of free carriers. The measured FX peak energy $\mathrm{E}_{\mathrm{FX}}(\mathrm{T})$ can be used to obtain the FX transition energy $\mathrm{E}_{\mathrm{x}}(\mathrm{T})$ in a particular sample by considering the temperaturedependent offset $\Delta \mathrm{E}=\mathrm{E}_{\mathrm{x}}(\mathrm{T})-\mathrm{E}_{\mathrm{FX}}(\mathrm{T})=\mathrm{kT} /(8 \ln 2)$. For each temperature $\mathrm{T}$, a free carrier concentration $\mathrm{n}(\mathrm{T})$ was directly measured or extrapolated from the Hall measurement, and used to calculate the band tailing and exchange interaction effects, and then the total bandgap shrinkage due to these effects. Assuming that the FX binding energy is independent of temperature, the ideal FX transition energy for a dilute sample is obtained using

$$
E_{x}(T, 0)=E_{x}(T, n)+E_{c}^{c^{*}}(n(T))+E_{c}^{e}(n(T))
$$

and fitting this $E_{x}(T, 0)$ versus $T$ is equivalent to fitting $E_{g}(T)$. Figure 4.14 shows the result of the $E_{x}(T)$ fitting for the Cermet undoped sample, using Manoogian-Wooley equation (Eq. 2.19) (Manoogian, 1984) with $\mathrm{U}=-5.5 \times 10^{-5} \mathrm{eV} / \mathrm{K}, \mathrm{s}=0.8, \mathrm{~V}=-5 \times 10^{-4} \mathrm{eV} / \mathrm{K}$, and $\theta_{\mathrm{E}}=$ $750 \mathrm{~K}$. Here, $\mathrm{U}$ was computed using $\mathrm{U}=-3 \mathrm{~B}\left(\partial \mathrm{E}_{\mathrm{g}} / \partial \mathrm{P}\right)<\alpha \mathrm{L}>$, with $\mathrm{B}=157 \mathrm{GPa},\left(\partial \mathrm{E}_{\mathrm{g}} / \partial \mathrm{P}\right)=$ $2.7 \times 10^{-6} \mathrm{eV} / \mathrm{bar}$, and $<\alpha \mathrm{L}>=4.3 \times 10^{-6} \mathrm{~K}^{-1}$ (Hamby, 2003). The fitted effective phonon Einstein temperature $\theta_{\mathrm{E}}$ is in very good agreement with the average energy ( $65 \mathrm{meV}$ ) of the top four $\Gamma$-point optical phonons in $\mathrm{ZnO}$. From the work of Schulz and Thiede (Schulz, 1987), the top four TO and LO phonons at $\Gamma$-point have frequencies 587, 576, 489, and 440 $\mathrm{cm}^{-1}$, and their average is $523 \mathrm{~cm}^{-1}$, corresponding to an average phonon energy of $64.8 \mathrm{meV}$, or an effective phonon temperature of $64.8 \mathrm{meV} /\left(8.62 \times 10^{-2} \mathrm{meV} / \mathrm{K}\right)=752 \mathrm{~K}$. Furthermore, this $\theta_{\mathrm{E}}$ value is important in temperature-dependent Hall mobility analysis. As will be discussed in Chapter 5, the polar optical phonon scattering, which can be expressed as a 
function of $\mathrm{T}_{\mathrm{po}} / \mathrm{T}$, sets up the intrinsic mobility of materials near and above RT. In the calculation of mobility associated with polar optical phonon scattering, the effective phonon temperature $T_{p o}=\theta_{E}=750 \mathrm{~K}$ was adopted instead of considering only the top LO phonon (72 meV) which gives $\mathrm{T}_{\mathrm{po}}=837 \mathrm{~K}$.

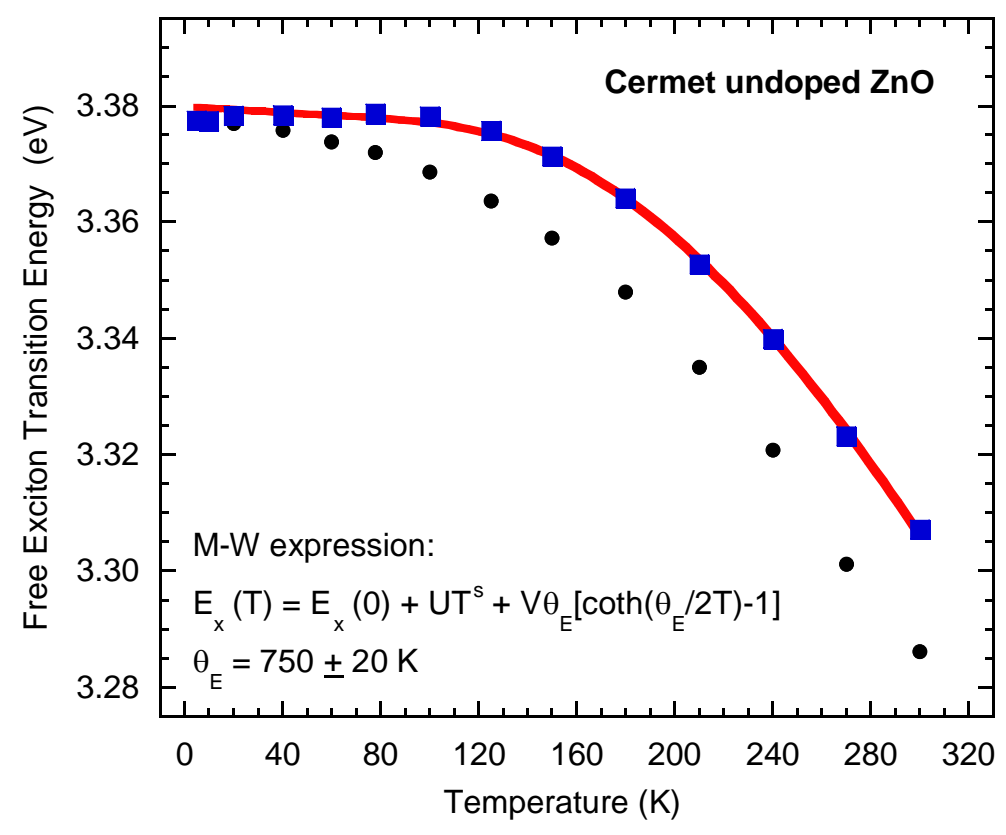

Figure 4.14 Free exciton transition energy $\mathrm{E}_{\mathrm{x}}$ (solid circles) obtained from FX PL measurement, and corrected for dilute free carrier case (squares). The solid red curve is a fit using ManoogianWoolley's equation (Eq. 2.19).

\subsection{Effect of Self-absorption on Photoluminescence in Transmission Geometry}

The PL experiments in this study were mostly excited with $325 \mathrm{~nm}$ laser, and the luminescence was collected in a reflection, (i.e., back-scattering) geometry, as shown in Fig. 3.1. With an estimated absorption coefficient $\alpha \approx 10^{4} \mathrm{~cm}^{-1}$ at $325 \mathrm{~nm}$, a penetration depth of about 1 micron was estimated for the laser light. The luminescence to be collected in the reflection geometry has only to escape a very thin layer of the sample before exiting the surface, and the self-absorption effect is not a big concern. In cases where the light is collected in transmission geometry, as with the $\mathrm{ZnO}: \mathrm{Ga}$ screen used in the associated particle detector in the deuterium-tritium (D-T) neutron generator (Fig. 1.5), the luminescence has to pass through the whole sample thickness, the effects of self-absorption on the UV edge 
emission can significantly change the observed PL intensity and peak positions, as has been shown in Fig. 1.4. In this case, a correction is needed to understand the nature of the observed spectra.

PL spectra from three single-crystal samples were taken using both reflection and transmission geometries, to evaluate the self-absorption effect on the edge and visible emissions. The incident laser in the transmission geometry was adjusted so as to introduce the same excitation power density as in the reflection geometry. For each sample, the same spot on the same face was illuminated in the two geometries.

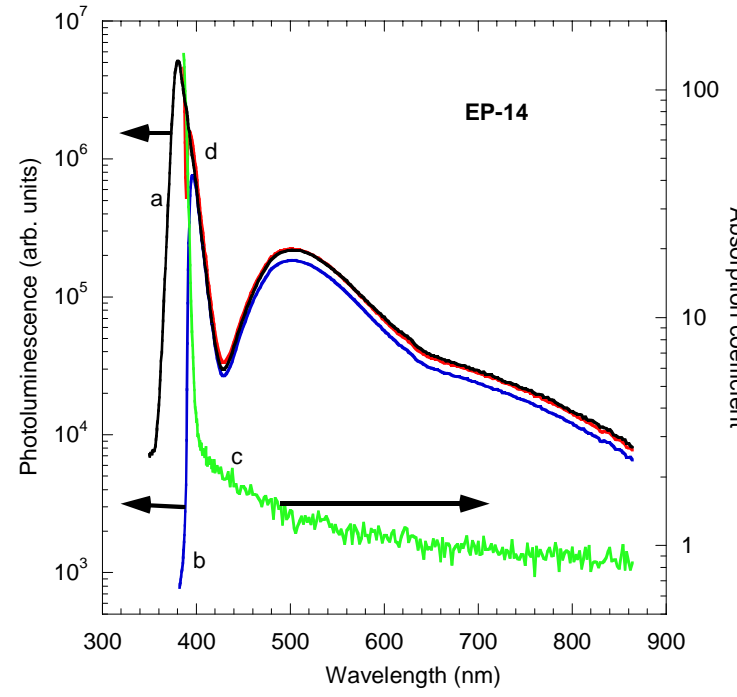

(a)

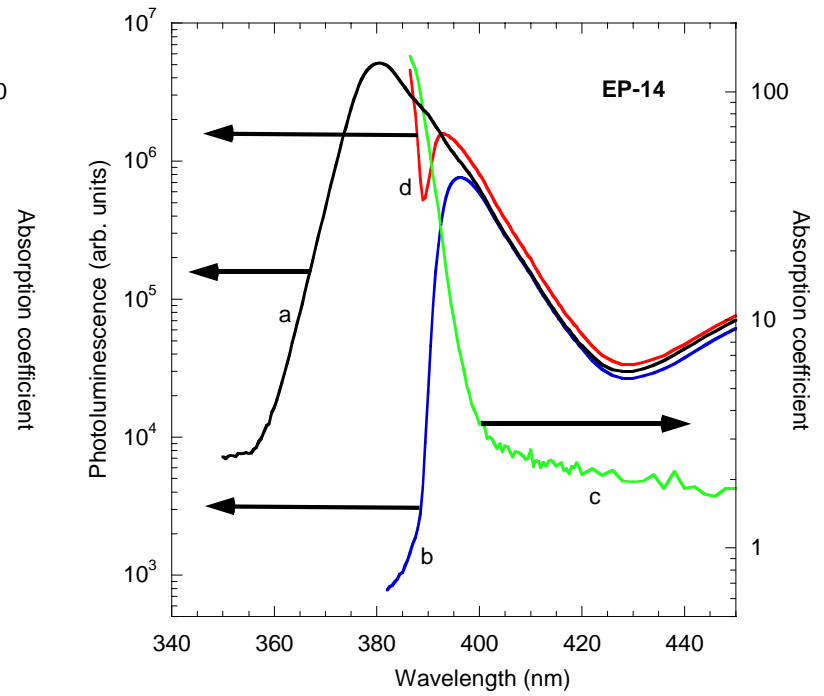

(b)

Figure 4.15 (a) 300-K PL spectra from sample EP-14 using reflection geometry (curve a, black), and transmission geometry (curve b, blue) shown along with the UV/vis absorption coefficient (curve c, green). Also shown is the calculated "real" PL (curve d, red). (b) An expanded view for the edge emission region. Notice the logarithmic vertical scales.

Figure 4.15 shows the 300-K PL spectra from an SCVT-grown sample EP-14 with a thickness of $\mathrm{t}=0.055 \mathrm{~cm}$ (from Eagle-Picher, Miami, OK) taken with reflection geometry (curve a, black) and transmission geometry (curve b, blue). The absorption coefficient $(\alpha)$ curve is also shown as curve $\mathrm{c}$ (green). The original absorbance data were taken using Cary14 system, and conversed to absorption coefficient data using Eq. 3.3, where reflective loss 
was accounted for with a uniform reflectance $\mathrm{R}=1 / 9$. .The reflection-geometry PL spectrum (curve a) has a UV edge emission peak at $380 \mathrm{~nm}(3.26 \mathrm{eV})$, associated with the first phonon-assisted free exciton transition (FX-1LO) (Wang, 2003). A broad green emission band occurs at longer wavelengths. The green band has a peak at $500 \mathrm{~nm}$ and is more than one order weaker in peak intensity than the edge emission. For the transmission geometry PL, the edge emission peak shifts to $396 \mathrm{~nm}$ (curve b), and the intensity of the shorter wavelength (higher energy) side of the edge emission band decreases very abruptly as wavelength decreases. This decrease corresponds to the fast increase in absorption coefficient (curve c). The maximum intensity of the UV emission peak is reduced by a factor of 7 compared to the reflection PL. From the transmission PL spectrum and absorption data, the "real" PL induced near the illuminated face was calculated by $I_{0}=I_{T} \exp (\alpha t) /(1-R)$ from Eq. 1.2. Here $\mathrm{I}_{0}$ is the luminescence intensity at the excitation site, and $\mathrm{I}_{\mathrm{T}}$ is the PL intensity collected in transmission geometry. The absorption coefficient $\alpha$ is in $\mathrm{cm}^{-1}$. The corrected PL spectrum is shown in Fig. 4.15 as curve d (red). The 400-860 nm region of curve d, corresponding to the transparent region of absorption spectrum, reproduces the reflection PL spectrum (curve a) very well. For wavelength shorter than $400 \mathrm{~nm}$, as seen in the expanded view in Fig. 4.15(b), the "real" PL spectrum (curve d) has a peak at a shorter wavelength $(393 \mathrm{~nm}$, or $3.154 \mathrm{eV})$. The peak energy of the reflection PL is $3.26 \mathrm{eV}(380 \mathrm{~nm})$, and is assigned to FX-1LO. The FX transition energy is $F_{x}=3.26+0.033=3.293 \mathrm{eV}$, and the peak energy of FX-2LO is $\mathrm{E}_{\mathrm{FX}-2 \mathrm{LO}}=\mathrm{E}_{\mathrm{x}}-2 \hbar \omega_{\mathrm{LO}}+(1 / 2) \mathrm{k}_{\mathrm{B}} \mathrm{T}=3.293-0.144+0.013=3.162 \mathrm{eV}$ $(392 \mathrm{~nm})$. Thus, the $396 \mathrm{~nm}$ peak of the transmission PL is mainly due to the FX-2LO transition, which suffers less self-absorption and survives the $0.55 \mathrm{~mm}$-thick sample. 
Figure 4.16 show the similar comparison from an as-grown HPM sample (CMT1) (Cermet), with a sample thickness of $\mathrm{t}=0.039 \mathrm{~cm}$. From the reflection geometry (curve a), the UV edge emission is the merged band of the FX transition and its phonon replica (FX1LO), with the FX claiming the peak at $378 \mathrm{~nm}(3.28 \mathrm{eV})$. The visible emission is weak and broad, with a peak around $620 \mathrm{~nm}$ and an intensity more than two orders weaker than the UV emission. For the transmission geometry PL (curve b), the edge emission peak shifts to 395 $\mathrm{nm}$, and its intensity is about 21 times less than the UV peak intensity obtained using

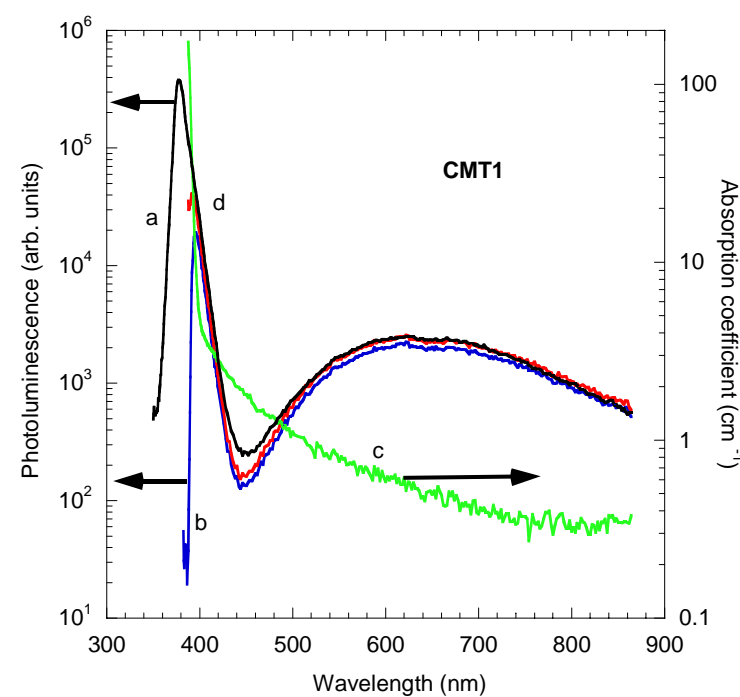

(a)

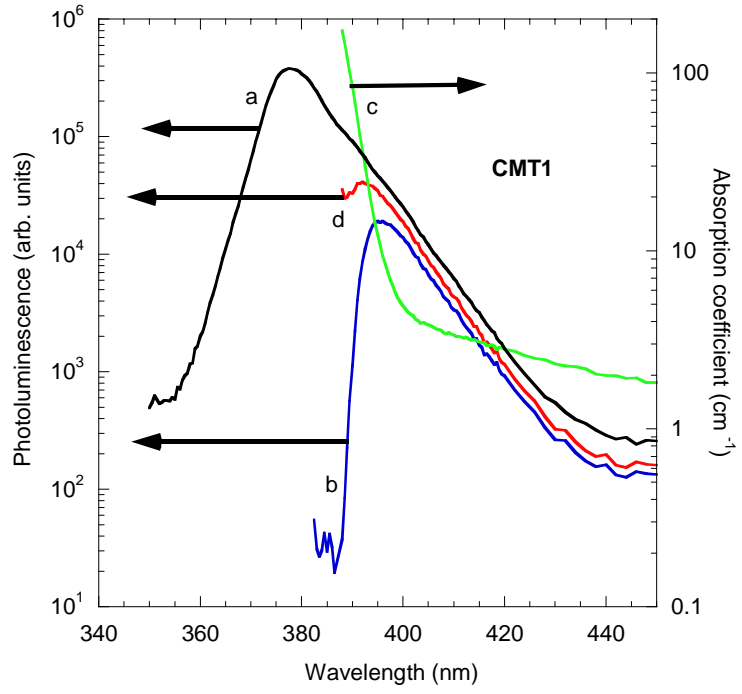

(b)

Figure 4.16 (a) 300-K PL spectra from sample CMT1 using reflection geometry (curve a, black), and transmission geometry (curve b, blue) shown along with the UV/vis absorption coefficient (curve c, green). Also shown is the calculated "real" PL (curve d, red). (b) An expanded view for the edge emission region. Notice the logarithmic vertical scales.

reflection geometry. From the transmission PL and the absorption spectrum (curve c), the "real" PL was calculated and shown as curve d. Similar to the case of sample EP-14, the calculated spectrum resembles the reflection PL spectrum for the wavelength region 500-860 nm. For the edge emission, the corrected spectrum has a peak at $392 \mathrm{~nm}(3.162 \mathrm{eV})$. As in the EP-14 sample, the FX-2LO transition survives the self-absorption effect. 
Figure 4.17 shows the PL spectra from the heavily doped Cermet $\mathrm{ZnO}$ :Ga sample (thickness $\mathrm{t}=0.028 \mathrm{~cm}$ ) using reflection (curve a) and transmission (curve b) geometries. The reflection PL has a UV peak at $378 \mathrm{~nm}$, which is associated with the direct $(\mathrm{e}, \mathrm{h})$ transition, as discussed in section 4.1. For the transmission PL, the UV peak appears at 396 $\mathrm{nm}$, and the intensity is about 11 times weaker than the reflection PL peak. Using the absorption coefficient (curve c), the transmission PL spectrum was corrected and shown as curve d. As can be seen from the expanded view in Fig. 4.17(b), the corrected PL has a UV peak at $394 \mathrm{~nm}(3.145 \mathrm{eV})$, corresponding to the predicted peak position of FX emission for this sample. So, differing from the two as-grown samples, it is the FX emission that survives the self-absorption in this heavily doped $\mathrm{ZnO}$ :Ga sample.

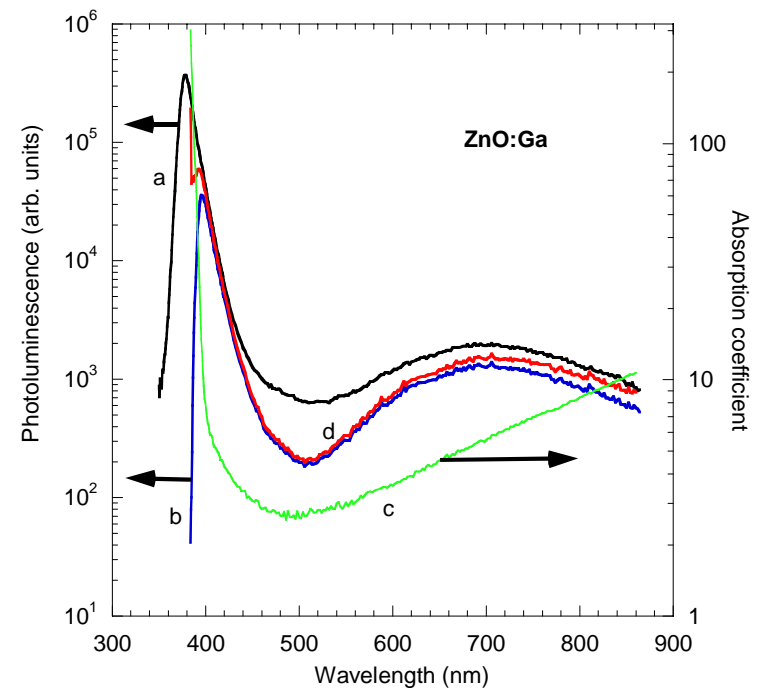

(a)

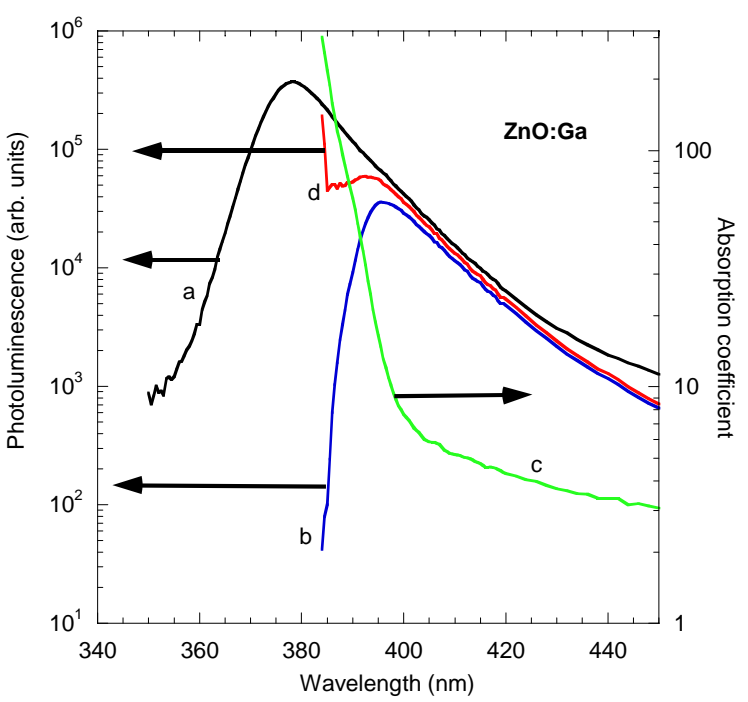

(b)

Figure 4.17 (a) 300-K PL spectra from sample Cermet $\mathrm{ZnO}$ :Ga using reflection geometry (curve a, black), and transmission geometry (curve b, blue) shown along with the UV/vis absorption coefficient (curve c, green). Also shown is the calculated "real" PL (curve d, red). (b) An expanded view for the edge emission region. Notice the logarithmic vertical scales.

In summary, PL spectra from as-grown and gallium doped $\mathrm{ZnO}$ single crystals were measured at temperatures from $300 \mathrm{~K}$ to $5 \mathrm{~K}$. PL line shape analysis and free-carrier related 
bandgap renormalization were used to determine the $300 \mathrm{~K}$ PL transition mechanism. The FX and FX-1LO transitions are responsible for the PL edge emission from the as-grown Cermet $\mathrm{ZnO}$, and the RT PL peak is attributed to the FX emission. Such attributions were supported by temperature dependent PL spectra from $5 \mathrm{~K}$ to $300 \mathrm{~K}$. Direct band-to-band (e,h) recombination is the major contributor to the RT UV emission from the heavily doped ZnO:Ga smple, but a side band at about $3.14 \mathrm{eV}$ may be attributed to FX transition. Thermal quenching of the integrated PL intensity $(350-420 \mathrm{~nm})$ from the $\mathrm{ZnO}$ :Ga sample is slower than that of the as-grown $\mathrm{ZnO}$, and is 4 times brighter than the latter at $300 \mathrm{~K}$, which is plausible for scintillator applications. Self-absorption effect was evaluated by comparison of PL spectra collected using reflection and transmission geometries from three single crystal samples. Absorption coefficient was used to correct the transmission PL spectra. It is conclusive that the lower-energy FX-2LO emission from the as-gown SCVT and HPM samples is more favorable than the FX and FX-1LO in surviving the self-absorption, while the FX emission, rather than the higher energy (e,h) emission, is able to escape from the heavily doped $\mathrm{ZnO}$ :Ga sample. 


\section{Chapter 5 Temperature-Dependent Hall Data Analysis}

In the preceding chapter, temperature-dependent Hall data were used with PL to determine the intrinsic bandgap temperature dependence of $\mathrm{ZnO}$. Most studies utilizing Hall data only take the measured quantities and do not attempt to perform a correction to understand the underlying scattering mechanisms. In this chapter, I present a detailed temperature-dependent Hall analysis of several n-type $\mathrm{ZnO}$ samples. The first section discusses the basic concept of temperature-dependent Hall Effect and Hall measurements. The second section describes the one-level n-fitting and the five-mechanism approach for Hall $\mathrm{r}$ factor calculations and Hall mobility fittings. The third section presents the fitting results and offers supportive results from other electrical and optical studies. In the last section, the five-mechanism approach is used to predict the intrinsic hole mobility and the total hole mobility behavior in doped p-type $\mathrm{ZnO}$. (The results for the Hall fitting of n-type $\mathrm{ZnO}$ have been accepted for publication in 2008 in Journal of Applied Physics.)

\subsection{Hall Effect Analysis: General Approach}

Room temperature (RT) Hall measurements can be used to establish whether a semiconducting sample is $\mathrm{n}$ type or $\mathrm{p}$ type from the sign of the measured Hall coefficient (Chapter 2). Also, one obtains information such as sheet and bulk conductivities, Hall mobility, and Hall density of free carriers. However, these properties vary with temperature and can be very different at temperatures either below or above RT. A temperaturedependent Hall effect analysis invoking several major intrinsic and extrinsic contributions to the scattering of free carriers can result in such knowledge as donor and acceptor concentrations and their energy levels within the band-gap. In this chapter, Hall effect 
measurements on n-type $\mathrm{ZnO}$ samples from different growth techniques were performed over the temperature range from 80 to $380 \mathrm{~K}$. In the analysis of mobility and free carrier concentration data, Hall $\mathrm{r}$ factor calculations were incorporated and the measured Hall data were corrected. In a partially compensated n-type sample, the charge balance equation gives (Look, 1987)

$$
n+N_{A}^{-}=N_{D}^{+}=\sum_{i} \frac{N_{D i}}{1+n / \phi_{D i}}
$$

Here $\mathrm{n}$ is the free carrier concentration and $\mathrm{N}_{\mathrm{A}}$ is the total density of acceptors. All acceptors are assumed to be ionized (i.e., $\mathrm{N}_{\mathrm{A}}{ }^{-}=\mathrm{N}_{\mathrm{A}}$ ). $\mathrm{N}_{\mathrm{Di}}{ }^{+}$represents the number of ionized donors out of the total density of $\mathrm{N}_{\mathrm{Di}}$ and is dependent on temperature through $\phi_{\mathrm{Di}}$.

$$
\phi_{\mathrm{Di}}=\beta_{\mathrm{i}} N_{\mathrm{c}} \exp \left(-\mathrm{E}_{\mathrm{Di}} / \mathrm{k}_{\mathrm{B}} \mathrm{T}\right)
$$

where $N_{\mathrm{c}}=4.829 \times 10^{15}\left(\mathrm{~m}^{*} / \mathrm{m}_{0}\right)^{3 / 2} \mathrm{~T}^{3 / 2}\left(\mathrm{~cm}^{-3}\right)$ is the effective density-of-states in the conduction band from Boltzmann statistics, and $\mathrm{m}^{*}$ is the rigid-lattice electron effective mass. $\mathrm{E}_{\mathrm{Di}}$ is the donor activation energy. In Eq. 5.2, $\beta_{\mathrm{i}}$ is the ratio of the spin degeneracy of the $i$ th donor states after ionization to that before ionization. For a hydrogenic donor before ionization, the single electron can occupy the ground state with either spin up or spin down, and $\beta=1 / 2$ is suitable.

If only one donor level is considered, Eq. 5.1 can be written as (Blakemore, 1987)

$$
\frac{n\left(n+N_{A}\right)}{N_{D}-n-N_{A}}=\phi_{D}
$$

Eq. 5.3 is a quadratic equation of $n$ and gives an explicit solution for $n$,

$$
n=(1 / 2)\left[\sqrt{\left.\left(N_{A}+\phi_{D}\right)^{2}+4\left(N_{D}-N_{A}\right) \phi_{D}\right)}-\left(N_{A}+\phi_{D}\right)\right]
$$


In n-type $\mathrm{ZnO}, \mathrm{m}^{*} / \mathrm{m}_{0}=0.24$ gives $\mathrm{N}_{\mathrm{c}} \approx 6.0 \times 10^{18} \mathrm{~cm}^{-3}$ at $300 \mathrm{~K}$. As an example case, assume that the activation energy of a group III shallow donor is about $40 \mathrm{meV}$. Using $\beta=1 / 2$, one obtains $\phi_{\mathrm{D}} \approx(1 / 2)\left(6.0 \times 10^{18}\right) \exp (-40 / 26) \approx 6.4 \times 10^{17} \mathrm{~cm}^{-3}$. For a lowcompensation $\left(\mathrm{N}_{\mathrm{A}}<<\mathrm{N}_{\mathrm{D}}\right)$, as-grown sample with typical shallow donor concentration in the range $\mathrm{N}_{\mathrm{D}}=10^{16}-10^{17} \mathrm{~cm}^{-3}$, then $\mathrm{N}_{\mathrm{D}}<<\phi_{\mathrm{D}}$ and

$$
n=(1 / 2)\left[\sqrt{\phi_{D}^{2}+4 N_{D} \phi_{D}}-\phi_{D}\right] \approx(1 / 2)\left[\phi_{D}\left(1+2 N_{D} / \phi_{D}\right)-\phi_{D}\right] \approx N_{D}
$$

That is, at $300 \mathrm{~K}$, almost all the shallow donors are ionized and contribute to free carriers. This means the sample is in the so-called saturation range of $\mathrm{n}$ as a function of $\mathrm{T}$, and the conductivity and mobility vary slowly, which is good for device operation. On the other hand, for intentionally doped cases, say, $\mathrm{N}_{\mathrm{D}}=1.2 \times 10^{18} \mathrm{~cm}^{-3} \approx 2 \phi_{\mathrm{D}}$, and one obtains $\mathrm{n} \approx$ $0.5 \mathrm{~N}_{\mathrm{D}}$ at $300 \mathrm{~K}$.

For heavily compensated cases, i.e., $\mathrm{N}_{\mathrm{D}} \approx \mathrm{N}_{\mathrm{A}}>$ n, Eq. 5.3 gives a simple solution of $\mathrm{n}=\phi_{\mathrm{D}}\left(\mathrm{N}_{\mathrm{D}}-\mathrm{N}_{\mathrm{A}}\right) /\left(\mathrm{N}_{\mathrm{A}}+\phi_{\mathrm{D}}\right)$. At higher temperatures, when $\phi_{\mathrm{D}}>>\mathrm{N}_{\mathrm{A}}$, then $\mathrm{n} \approx \mathrm{N}_{\mathrm{D}}-\mathrm{N}_{\mathrm{A}}$. Once again, the free-carrier density reaches a saturation stage.

When a donor ionizes, it may give out the electron from one of the excited states, as occurs in a hydrogen atom. Taking into account ionizations from exited states, Eq. 5.3 can be written as (Blakemore, 1987)

$$
\frac{n\left(n+N_{A}\right)}{N_{D}-n-N_{A}}=\frac{\phi_{D}}{1+F}
$$

Here F describes the contributions of excited impurity states and is a function of temperature. In a simple hydrogenic model, $\mathrm{F}$ has the form

$$
F=4 \exp \left(-\frac{3}{4} \frac{E_{D}}{k_{B} T}\right)+9 \exp \left(-\frac{8}{9} \frac{E_{D}}{k_{B} T}\right)+16 \exp \left(-\frac{15}{16} \frac{E_{D}}{k_{B} T}\right)+\cdots
$$


It is only necessary to consider the first few terms in Eq. 5.7, since the higher excited states will form part of the conduction band and are no longer distinguishable. Figure 5.1 shows the temperature dependence of $\mathrm{F}$ for several different $\mathrm{E}_{\mathrm{D}}$ values, considering only the first three terms in Eq. 5.7. As can be seen in Fig. 5.1, the F-value is large compared to 1 for shallow levels and at higher temperatures. In these cases, ignoring the effect of excited states may cause serious error in the fitted parameters.

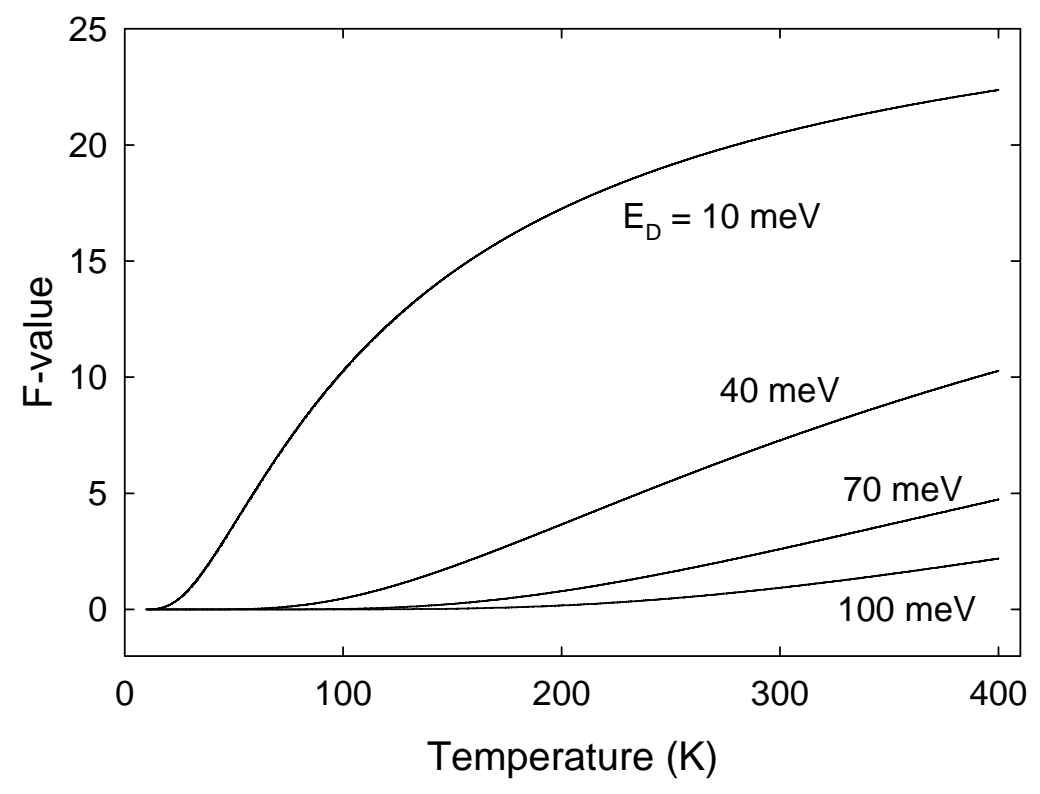

Figure 5.1. F-value for different donor levels calculated by considering only the first three terms in Eq. 5.7.

Using Eq. 5.3 to analyze free-carrier data ( $\mathrm{n}$ vs. T), one can obtain fitting parameters $\mathrm{N}_{\mathrm{D}}, \mathrm{N}_{\mathrm{A}}, \mathrm{E}_{\mathrm{D}}$ and $\beta$. The latter two values are especially important in identifying the donor(s). In $\mathrm{ZnO}, \mathrm{Al}$ and $\mathrm{Ga}$ appear to be the most common shallow donors in as-grown samples, as have been supported by other methods such as $5-\mathrm{K}$ PL and EPR. In these cases, $\beta=1 / 2$ is appropriate for a $\mathrm{D}^{0} \rightarrow \mathrm{D}^{+}$ionization. Deep donor levels associated with transition metal (TM) ions such as $\mathrm{Co}, \mathrm{Mn}$, and $\mathrm{Fe}$, have also been found in $\mathrm{n}$-type samples. $\beta$ values other than $1 / 2$ may be more appropriate to account for the degeneracy relation before and after the ionization of a certain donor state of the TM ions. 
With the donor activation energy $E_{D}$ and the donor concentration $N_{D}$, the ionization energy $\mathrm{E}_{\mathrm{D} 0}$ of isolated donor centers is evaluated by taking into account the Coulomb screening effect (Pearson, 1949)

$$
\mathrm{E}_{\mathrm{D} 0}=\mathrm{E}_{\mathrm{D}}+\mathrm{C} \mathrm{N}_{\mathrm{D}}^{1 / 3}
$$

where $\mathrm{C}=2.66 \mathrm{e}^{2} / \mathrm{k} \approx 3.90 \times 10^{-4} / \mathrm{k}(\mathrm{meV}-\mathrm{cm})$. For $\mathrm{ZnO}$, the relative dielectric constant $\mathrm{k}=$ 7.8, and $\mathrm{C}=5.0 \times 10^{-5}(\mathrm{meV}-\mathrm{cm})$. This is important especially for high n-type samples, since the donor band is very close to the conduction band minimum and the activation energy could be very small. This small energy value may be misleading in identifying the associated donor. For example, a donor level found from Eq. 5.3 with $E_{D}=35 \mathrm{meV}$ and $\mathrm{N}_{\mathrm{D}}=1 \times 10^{17}$

$\mathrm{cm}^{-3}$ has an ionization energy of $\mathrm{E}_{\mathrm{D} 0}=58 \mathrm{meV}$. The same activation energy level with $\mathrm{N}_{\mathrm{D}}=$ $1 \times 10^{18} \mathrm{~cm}^{-3}$ gives a larger ionization energy of $\mathrm{E}_{\mathrm{D} 0}=85 \mathrm{meV}$.

\subsection{Intrinsic Hall $r$ Factor and Intrinsic Mobility in ZnO}

As in other II-VI materials, the major contributors in limiting the sample mobility in $\mathrm{ZnO}$ include both intrinsic and extrinsic scattering mechanisms. Intrinsic scattering mechanisms refer to inelastic intraband scatterings via acoustic and optical phonons, namely piezoelectric scattering (pe), deformation potential scattering (dp), and polar optical phonon scattering (po). Extrinsic scatterings refer to elastic interactions between free electrons and defects, especially ionized impurity scattering (ii) and neutral impurity scattering (ni). Other mechanisms such as dislocation scattering and hopping effects can also become major limiting factors in some samples at low temperatures. In this study, by combining po, pe and dp scatterings, a limit of temperature-dependent intrinsic mobility for a pure $\mathrm{ZnO}$ sample is established. Using this limit to fit the measured Hall mobility data from samples grown by 
different techniques, the densities of donors and acceptors were obtained, which were then compared with the results from the free-carrier concentration analysis to understand the impurity levels in each sample. These results were also compared with PL, EPR and FTIR spectra analyses to identify the defects. The relaxation-time approximation (RTA) approach and Matthiessen's rule were employed in calculating the Hall $\mathrm{r}$ factors and correcting measured Hall data.

During the scattering of an electron by the lattice field, the conservation of both energy and momentum requires the release of a phonon. For small energy changes, the associated acoustic phonon induces lattice deformations and the scattering is called deformation potential (dp) scattering. The relaxation time related to dp scattering is

$$
\tau_{d p}=\frac{\pi \hbar^{4} \rho s^{2}}{\sqrt{2} E_{1}^{2} m_{p}^{* 3 / 2}\left(k_{B} T\right)} E^{-1 / 2}
$$

where $\mathrm{E}$ is electron energy with respect to the conduction-band minimum, $\mathrm{T}$ is temperature, and $\mathrm{m}_{\mathrm{p}}{ }^{*}$ is the electron polaron effective mass. $\rho$ is the crystal density and $\mathrm{s}$ is the average velocity of sound in the material. The product $\rho s^{2}=\mathrm{c}_{l}$ is also called the longitudinal elastic coefficient. $E_{1}$ is the acoustic phonon deformation potential. The experimental value of $E_{1}$ is $3.8 \mathrm{eV}$ for $\mathrm{ZnO}$, but a value of $15 \mathrm{eV}$ has been obtained as a fitting parameter and adopted by many other researchers. In this work, we use the experimental value of $E_{1}$ so that the intrinsic mobility from dp scattering will not change from sample to sample. Taken separately from all other scattering mechanisms, dp scattering gives a mobility limit of

$$
\mu_{d p}=\frac{e\left\langle\tau_{d p}\right\rangle}{m_{p}^{*}}=\frac{\sqrt{8 \pi} \hbar^{4} \rho s^{2} e}{3 E_{1}^{2} m_{p}^{* / 2}\left(k_{B} T\right)^{3 / 2}}
$$


From Eq. 5.10, $\mu_{\text {dp }}$ has a temperature dependence of $\mathrm{T}^{-3 / 2}$, and could be important at high temperatures in a given material depending on the $\mathrm{E}_{1}$ value. However, compared with III-V compounds (e.g., $\mathrm{E}_{1}$ is $8.4 \mathrm{eV}$ for $\mathrm{GaN}$, and $8.6 \mathrm{eV}$ for $\mathrm{GaAs}$ ), II-VI materials usually have relatively smaller $E_{1}$ values in the range from 3 to $5 \mathrm{eV}$. Since $\mu_{\mathrm{dp}} \alpha \mathrm{E}_{1}^{-2}$, the smaller $\mathrm{E}_{1}$ values make dp scattering a less important mechanism in II-VI compounds.

In ionic semiconductors, the electron-lattice interaction also induces an electric field, known as the piezoelectric effect. This process may involve either a longitudinal or a transverse acoustic phonon, and is called piezoelectric (pe) potential scattering. The associated relaxation time and mobility are given as

$$
\begin{aligned}
& \tau_{p e}=\frac{\sqrt{8} \pi \hbar^{2} \varepsilon}{e^{2} P^{2} m_{p}^{* 1 / 2}\left(k_{B} T\right)} E^{1 / 2} \\
& \mu_{p e}=\frac{e\left\langle\tau_{p e}\right\rangle}{m_{p}^{*}}=\frac{16 \sqrt{2 \pi} \hbar^{2} \varepsilon}{3 e P^{2} m_{p}^{* 3 / 2}\left(k_{B} T\right)^{1 / 2}}
\end{aligned}
$$

where $\mathrm{P}$ is the piezoelectric coupling coefficient, and $\varepsilon$ is the static permittivity. In most III$\mathrm{V}$ semiconductors (except for $\mathrm{GaN}$ ), the $\mathrm{P}$ values are small and pe scattering is not as important as dp scattering in limiting the sample mobility. However, many II-VI materials, especially $\mathrm{ZnO}$, have large $\mathrm{P}$ values (and small $\mathrm{E}_{1}$ values, as discussed above), and the pe scattering often dominates dp scattering.

For wurtzite materials, the piezoelectric coupling coefficient $\mathrm{P}$ usually reflects the anisotropy in the piezoelectric interaction by having two distinct values, $\mathrm{P}_{\perp}$ and $\mathrm{P}_{\|}$, with respect to the electric field during a transport measurement. For $\mathrm{ZnO}, \mathrm{P}_{\perp}=0.21$ and $\mathrm{P}_{\|}=$ 0.36 have been reported (Rode, 1970). These values can be calculated from the independent elements of the piezoelectric stress tensor $\boldsymbol{h}$ (Zook, 1963; Zook, 1964). 


$$
\boldsymbol{h}=\left\{\begin{array}{cccccc}
0 & 0 & 0 & 0 & h_{15} & 0 \\
0 & 0 & 0 & h_{15} & 0 & 0 \\
h_{31} & h_{31} & h_{33} & 0 & 0 & 0
\end{array}\right\}
$$

using

$$
\begin{aligned}
& P_{\perp}^{2}=4 \varepsilon\left(21 h_{15}^{2}+6 h_{15} h_{x}+h_{x}^{2}\right) / 105 c_{t}+\varepsilon\left(21 h_{33}^{2}-24 h_{33} h_{x}+8 h_{x}^{2}\right) / 105 c_{l} \\
& P_{\|}^{2}=2 \varepsilon\left(21 h_{15}^{2}+18 h_{15} h_{x}+5 h_{x}^{2}\right) / 105 c_{t}+\varepsilon\left(63 h_{33}^{2}-36 h_{33} h_{x}+8 h_{x}{ }^{2}\right) / 105 c_{l}
\end{aligned}
$$

where

$$
\begin{gathered}
h_{\mathrm{x}}=h_{33}-h_{31}-2 h_{15} . \\
c_{l}=\left(8 c_{11}+4 c_{13}+3 c_{33}+8 c_{44}\right) / 15, \\
c_{t}=\left(2 c_{11}-4 c_{13}+2 c_{33}+7 c_{44}\right) / 15,
\end{gathered}
$$

The $c_{l}$ and $c_{t}$ are the longitudinal and transverse elastic constants, respectively. Out of the six independent elastic constants $c_{\mathrm{ij}}$, only four are piezoelectric active. From the reported values for $\mathrm{ZnO}$ (Bateman, 1962) of $c_{\mathrm{ij}}\left(\right.$ in $\left.10^{12} \mathrm{dyn} / \mathrm{cm}^{2}\right), c_{11}=2.097, c_{13}=1.051, c_{33}=2.109$, and $c_{44}=0.4247$. Eqs. 5.17-18 give $c_{l}=2.0$ and $c_{t}=0.49$ for $\mathrm{ZnO}$.

The piezoelectric tensor $\boldsymbol{h}$ is related to the dielectric piezoelectric stress tensor $\boldsymbol{e}$ and permittivity tensor $\varepsilon^{\mathrm{S}}$ (measured with constant strain) as $\boldsymbol{e}=\boldsymbol{\varepsilon}^{\mathrm{S}} \boldsymbol{h}$. For wurtzite structure materials such as $\mathrm{ZnO}$, this relation involves only a few non-zero elements:

$$
\left(\begin{array}{cccccc}
0 & 0 & 0 & 0 & e_{15} & 0 \\
0 & 0 & 0 & e_{15} & 0 & 0 \\
e_{31} & e_{31} & e_{33} & 0 & 0 & 0
\end{array}\right)=\left(\begin{array}{ccc}
\varepsilon_{11} & 0 & 0 \\
0 & \varepsilon_{11} & 0 \\
0 & 0 & \varepsilon_{33}
\end{array}\right)\left(\begin{array}{cccccc}
0 & 0 & 0 & 0 & h_{15} & 0 \\
0 & 0 & 0 & h_{15} & 0 & 0 \\
h_{31} & h_{31} & h_{33} & 0 & 0 & 0
\end{array}\right)
$$

Solving Eq. 5.19, the three non-zero piezoelectric stress tensors of $\boldsymbol{h}$ can be calculated using $h_{15}=e_{15} / \varepsilon_{11}, h_{31}=e_{31} / \varepsilon_{33}$, and $h_{33}=e_{33} / \varepsilon_{33}$.

The temperature dependence of $\mathrm{P}$ is ignored in Eqs. 5.11-12. However, the dielectric piezoelectric stress elements $\left(\mathrm{e}_{\mathrm{ij}}\right)$ and the permittivity elements $\left(\varepsilon_{\mathrm{ij}}{ }^{\mathrm{S}}\right)$ are all temperature- 
dependent (Kobiakov, 1980; Hill, 2003). The dielectric piezoelectric stress elements decrease and the permittivity elements increase as temperature increases. Given the range of reported room temperature values of $\mathrm{e}_{\mathrm{ij}}$ and $\varepsilon_{\mathrm{ij}} \mathrm{S}$, ranges in the numerical values of $\mathrm{P}$ are obtained: $\mathrm{P}_{\perp}=0.16$ to 0.29 and $\mathrm{P}_{\|}=0.24$ to 0.42 . Based on the temperature dependent change of $\mathrm{e}_{\mathrm{ij}}$ and $\varepsilon_{\mathrm{ij}}^{\mathrm{S}}$, using their reported RT values (Landolt-Börnstein, 1987) and $\varepsilon_{\mathrm{r}}=7.8$ for $\mathrm{ZnO}$, and considering a $15 \%$ increase for $\mathrm{e}_{\mathrm{ij}}$ at $100 \mathrm{~K}$ compared to $300 \mathrm{~K}$, and a $10 \%$ decrease for $\varepsilon_{\mathrm{ij}}{ }^{\mathrm{S}}, \mathrm{P}_{\perp}=0.25$ and $\mathrm{P}_{\|}=0.46$ were calculated from Eq. 5.14-15. The 100-K value $\mathrm{P}_{\perp}=0.25$ was then used as an average for the whole temperature range in Eqs. 5.11-12, instead of the commonly accepted RT value of $\mathrm{P}_{\perp}=0.21$ (Rode, 1975). This slightly larger value for $\mathrm{P}_{\perp}$ reduces the predicted mobility for pe scattering by about $30 \%$, and fits the mobility data better for $\mathrm{T}<200 \mathrm{~K}$ in two relatively pure $\mathrm{ZnO}$ samples, as will be discussed latter in the results section. For temperatures around or above RT, this $30 \%$ reduction is not a significant factor since the polar optical phonon scattering is the most important limiting mechanism for that range.

Optical phonons scatter free electrons through the induced macroscopic polarized electric field. One of these processes is called the Fröhlich interaction, which involves the high-energy PO phonons and will be a dominant limiting factor at relatively higher temperatures. For polar optical (po) phonon scattering, the relaxation time is given by

$$
\tau_{p o}=\frac{\sqrt{8} \pi \hbar^{2}\left(e^{T_{p o} / T}-1\right) \chi\left(T_{p o} / T\right)}{e^{2}\left(k_{B} T_{p o}\right) m_{p}{ }^{* 1 / 2}\left(\varepsilon_{\infty}^{-1}-\varepsilon^{-1}\right)} E^{1 / 2} \quad\left(0 \leq \mathrm{T}_{\mathrm{po}} / \mathrm{T} \leq 5\right)
$$

or

$$
\tau_{p o}=\frac{\sqrt{8} \pi \hbar^{2}\left(e^{T_{p o} / T}-1\right)}{e^{2}\left(k_{B} T_{p o}\right)^{1 / 2} m_{p}{ }^{* 1 / 2}\left(\varepsilon_{\infty}^{-1}-\varepsilon^{-1}\right)} \quad\left(5 \leq \mathrm{T}_{\mathrm{po}} / \mathrm{T} \leq \infty\right)
$$


where $\varepsilon$ and $\varepsilon_{\infty}$ are the static and high-frequency permittivities, respectively. $\mathrm{T}_{\mathrm{po}}$ is the effective polar optical phonon temperature, and $\chi\left(T_{p o} / T\right)$ is a slowly varying function of $T$ (Putley, 1968).

In $\mathrm{ZnO}$, the highest $\mathrm{LO}$ phonon energy is $\hbar \omega_{\mathrm{LO}}=72 \mathrm{meV}$, which gives $\mathrm{T}_{\mathrm{po}}=72 / 0.086$ $=837 \mathrm{~K}$. This value is far two large for temperature ranges in a typical transport measurement, as has concerned other groups (Makino, 2006). In this work, we choose the four highest $\Gamma$-point optical phonons in $\mathrm{ZnO}$ and use their average energy $64.8 \mathrm{meV}$ to get an effective po phonon temperature $\mathrm{Tpo}=750 \mathrm{~K}$. This choice is supported by temperaturedependent PL data from an undoped $\mathrm{ZnO}$ sample and the bandgap renormalization analysis, as discussed in Chapter 4, where fitting of the temperature dependent bandgap data gave an Einstein temperature $\theta_{\mathrm{E}}=750 \pm 20 \mathrm{~K}$.

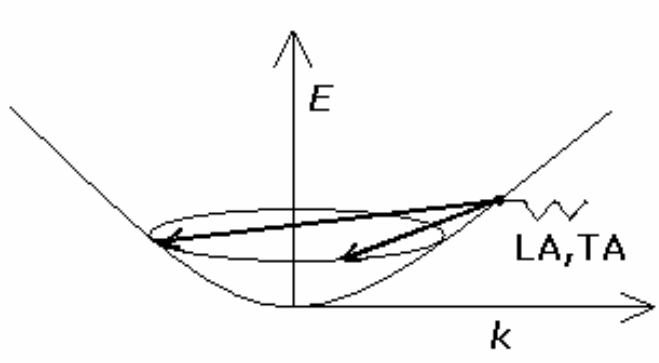

(a)

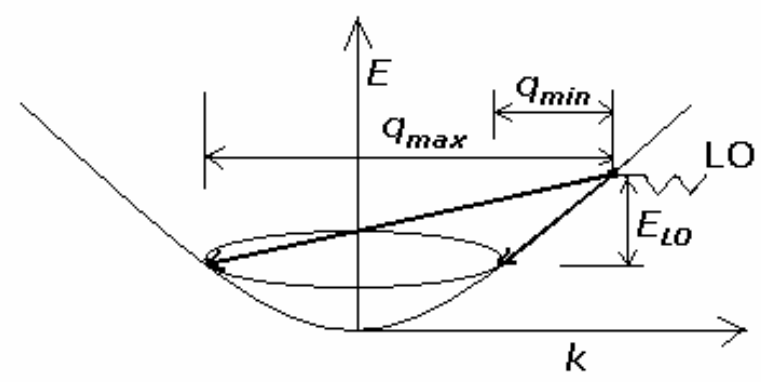

(b)

Figure 5.2. Schematic diagram for electron scattering in a parabolic band with the emission of (a) an acoustic phonon, or (b) an longitudinal optical (LO) phonon (from Yu, 2001).

Figure 5.2 is a schematic diagram of electron scattering processes involving acoustic phonons (a) and optical phonons (b). Scattering by an acoustic phonon causes only a small change in the electron energy, while scattering by an optical phonon, typically the LO phonon, causes large energy changes, usually by several tens of meV.

Polaron masses, instead of rigid-lattice masses, are used in Eqs. 5.9-12 and 5.20-21. 
For a II-VI semiconductor, the Fröhlich electron-phonon coupling constant $\alpha$ (see Eq. 2.9) is much larger than that of a III-V or a group IV semiconductor (Mahan, 1972). This results in a significant modification to the effective mass, according to $\mathrm{m}_{\mathrm{p}}{ }^{*}=\mathrm{m}_{\mathrm{e}}{ }^{*} /(1-\alpha / 6)$. Here, $\mathrm{m}_{\mathrm{p}}{ }^{*}$ and $\mathrm{m}_{\mathrm{e}}{ }^{*}$ are effective polaron and rigid-lattice electron masses, respectively. For GaAs, $\alpha=$ 0.06 , and the difference between $\mathrm{m}_{\mathrm{p}}{ }^{*}$ and $\mathrm{m}_{\mathrm{e}}{ }^{*}$ is only about $1 \%$. For $\mathrm{ZnO}$, on the other hand, $\alpha=1$ for electrons and $\mathrm{m}_{\mathrm{p}}{ }^{*}=1.2 \mathrm{~m}_{\mathrm{e}}^{*}$.

By including the above three electron-lattice interactions, the intrinsic Hall $r$ factor could be calculated from

$$
\mathrm{r}_{\mathrm{H}}^{\text {int }}=<\tau_{\text {int }}^{2}>/<\tau_{\text {int }}>^{2}
$$

where $\tau_{\text {int }}$ is the intrinsic relaxation time determined by Matthiessen's rule,

$$
\tau_{\text {int }}{ }^{-1}=\tau_{\mathrm{po}}{ }^{-1}+\tau_{\mathrm{pe}}{ }^{-1}+\tau_{\mathrm{dp}}{ }^{-1}
$$

From Eqs. 5.9, 5.11, and 5.20-21, the relaxation times of three intrinsic scattering mechanisms have different energy dependences. Taken separately, po scattering introduces a Hall $\mathrm{r}$ factor of $\mathrm{r}=1\left(5 \leq \mathrm{T}_{\mathrm{po}} / \mathrm{T} \leq \infty\right)$, or $\mathrm{r}=45 \pi / 128 \approx 1.1\left(0 \leq \mathrm{T}_{\mathrm{po}} / \mathrm{T} \leq 5\right)$, pe scattering gives $r=45 \pi / 128 \approx 1.1$ while dp scattering introduces a Hall $r$ factor $r=3 \pi / 8 \approx 1.2$. The value of $E_{1}$ used in a Hall mobility analysis can influence the relative weight of the dp contribution. In $\mathrm{ZnO}, \tau_{\mathrm{dp}}$ is about two orders larger than $\tau_{\mathrm{pe}}$ (at low temperature) or $\tau_{\mathrm{po}}$ (at high temperature), hence the modification to the intrinsic Hall $r$ factor $\left(r_{H}{ }^{\text {int }}\right)$ is trivial. Using Eqs. 5.22-23, $\mathrm{r}_{\mathrm{H}}^{\text {int }}$ was found to vary from 1.09 to 1.00 for temperatures from $60-400 \mathrm{~K}$. The intrinsic mobility can then be calculated using $\mu_{\text {int }}=<\tau_{\text {int }}>/ \mathrm{m}_{\mathrm{p}}{ }^{*}$ with the result of Eq. 5.22. Figure 5.3 shows the predicted temperature dependences of intrinsic electron mobility $\mu_{\text {int }}$ in n-type $\mathrm{ZnO}$ from 60 - $400 \mathrm{~K}$, along with the separate contributions of pe, po and dp scatterings. Among the intrinsic mechanisms, po scattering limits sample mobility in the 
higher temperature regime, while piezoelectric scattering is important at lower temperatures. The results shown in Fig.5.3 correspond to the behaviors associated with the true carrier mobility in ideal, or pure, samples. The predicted room temperature (RT) intrinsic mobility $\mu_{\text {int }}=201 \mathrm{~cm}^{2} / \mathrm{Vs}$ for n-type $\mathrm{ZnO}$. By including the calculated intrinsic Hall $\mathrm{r}$ factor, $\mathrm{r}_{\mathrm{H}}{ }^{\text {int }}$ $=1.1$ at $300 \mathrm{~K}$, the RT Hall mobility for electrons in $\mathrm{ZnO}$ is $\mu_{\mathrm{H}}=220 \mathrm{~cm}^{2} / \mathrm{Vs}$. At lower temperatures, the intrinsic mobility is limited mainly by pe scattering, which gives $\mu_{\text {int }}=$ $2600 \mathrm{~cm}^{2} / \mathrm{Vs}$ and $\mu_{\mathrm{H}}=2850 \mathrm{~cm}^{2} / \mathrm{Vs}$ at $60 \mathrm{~K}$ for $\mathrm{ZnO}$. The highest reported Hall mobility at $300 \mathrm{~K}$ measured from a very pure $\mathrm{ZnO}$ sample grown by the SCVT method is $\mu_{\mathrm{H}}=205$ $\mathrm{cm}^{2} / \mathrm{Vs}$ (Look, 1998). In an earlier work, a peak Hall mobility $\mu_{\perp} \sim 2500 \mathrm{~cm}^{2} / \mathrm{Vs}$ at $60 \mathrm{~K}$ has been reported (Rode, 1975).

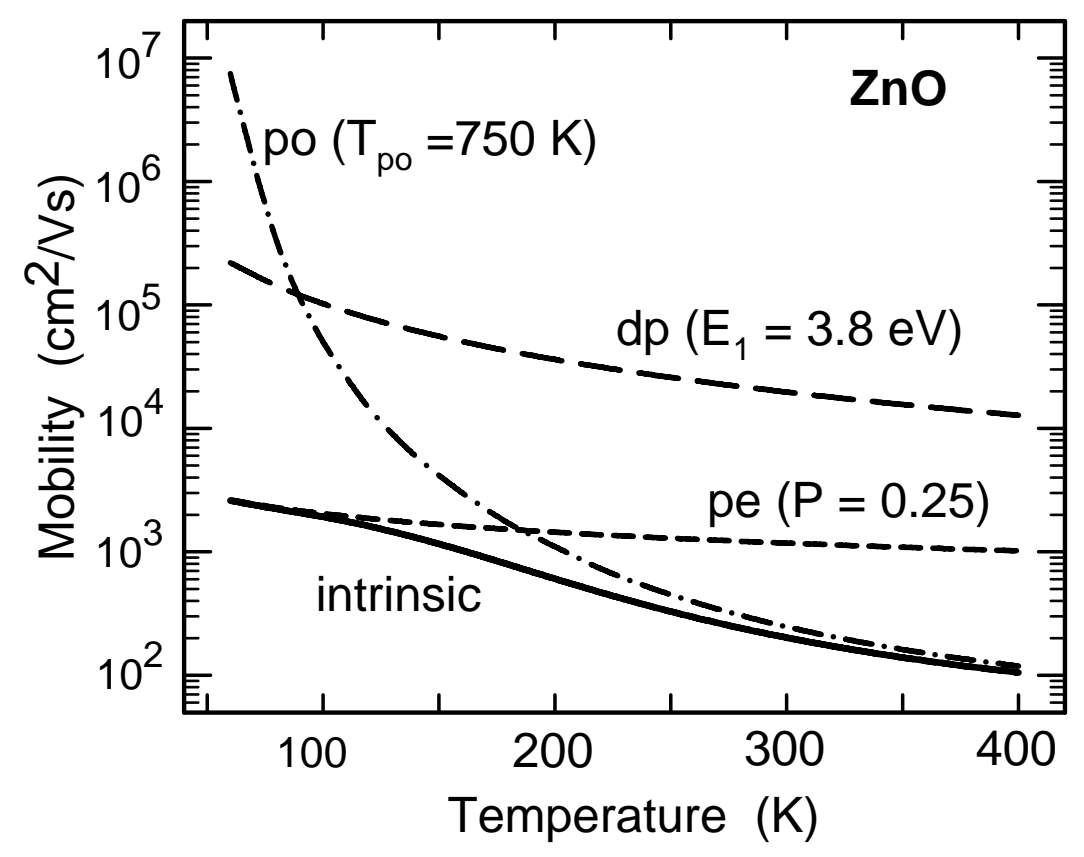

Figure 5.3. Intrinsic mobility prediction for $\mathrm{n}$ type $\mathrm{ZnO}$ (solid line) for the temperature range 60 -400 by including the polar optical phonon scattering (po), piezoelectric scattring (pe), and deformation potential scattering $(\mathrm{dp})$. The parameters used in Eqs. (5.8-12) and (5.2021) are indicated. Notice the logarithmic vertical scale.

\subsection{Impurity Scattering and Mobility Fitting}

Now that the intrinsic mobility limit is set up for ideal n-type samples, one can fit the measured mobility data by including the extrinsic scattering mechanisms in conjunction with 
Hall $r$ factor $\left(r_{H}\right)$ calculations, following an iterative approach (Look, 1989).

Ionized impurities scatter electrons through the screened coulomb potential

$$
\Delta V=\frac{Z e^{2}}{4 \pi \varepsilon r} e^{-r / \lambda_{D}}
$$

where $\lambda_{D}$ is the Debye screening length. In the non-degenerate case, $\lambda_{D}{ }^{2}=\varepsilon k_{B} T / e^{2} n$ for freeelectron screening. If donors are not completely ionized in a compensated sample,

$$
\lambda_{D}^{2}=\frac{\varepsilon k_{B} T}{e^{2}\left[n+\left(n+N_{A}\right)\left(1-\frac{n+N_{A}}{N_{D}}\right)\right]}
$$

In the Brooks-Herring approach, the ionized-impurity scattering cross section is given by

$$
\sigma(\Theta)=\left[\frac{K}{2 \sin ^{2} \frac{\Theta}{2}+\left(\frac{q_{0}}{2 k}\right)^{2}}\right]^{2}
$$

where $\Theta$ is the scattering angle as shown in Fig. 5.4, $K=\mathrm{Ze}^{2} /\left(\mathrm{mv}_{\mathrm{k}}{ }^{2}\right)$ is a characteristic distance determined by the electron energy, and is related to the scattering angle $\Theta$ and impact parameter $\mathrm{b}$ as $\mathrm{b}=\mathrm{K} \cot (\Theta / 2) . \mathrm{q}_{0}=1 / \lambda_{\mathrm{D}}$ is the screening wave vector, and $k$ is the effective wave vector of the incident electron. In this approach, the effects of cation and anion ions on the electron are not distinguished.

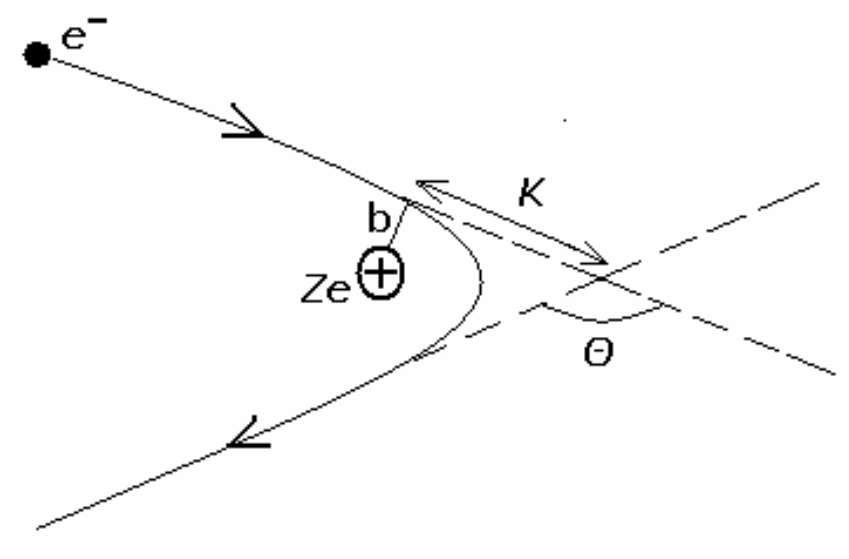

Figure 5.4. A schematic sketch of an electron scattered by a positively charged impurity ion. $K$ is the characteristic distance and $b$ is the impact parameter (from P. Y. Yu, 2001). 
From this scattering cross section, the relaxation time associated with ii scattering for the non-degenerate case is given by:

$$
\tau_{i i}^{n o n-\mathrm{deg}}=\frac{16 \sqrt{2} \pi \varepsilon^{2} m_{p}^{*^{1 / 2}} E^{3 / 2}}{N_{I} Z^{2} e^{4}[\ln (1+y)-y /(1+y)]}
$$

where Ze is the ionic charge of the impurity, $N_{I}=n+2 N_{a}$ is the total density of ionized impurities assuming that all acceptors are ionized in n-type material, and $\mathrm{n}$ is the free carrier concentration. The inclusion of ii scattering provides a means to determine the acceptor concentration $\mathrm{N}_{\mathrm{a}}$. In the denominator of Eq. 5.27, the function $\mathrm{y}$ is

$$
\mathrm{y}=8 \varepsilon \mathrm{m}_{\mathrm{p}}^{*}\left(\mathrm{k}_{\mathrm{B}} \mathrm{T}\right) E / \mathrm{h}^{2} \mathrm{e}^{2} \mathrm{n}=4.99 \times 10^{17} \mathrm{k}\left(\mathrm{m}_{\mathrm{p}}{ }^{*} / \mathrm{m}_{0}\right) E \mathrm{~T} / \mathrm{n}
$$

where $\mathrm{k}$ is the relative static dielectric constant and $E$ the electron energy in $\mathrm{eV}$. When $\mathrm{y}>>$ 1, Eq. 5.27 is valid.

In the case of degenerate statistics, the energy dependence in $\tau_{\mathrm{ii}}$ can be simplified as $E$ $\approx E_{F}=\left(\hbar^{2} / 2 \mathrm{~m}^{*}\right)\left(3 \pi^{2} \mathrm{n}\right)^{2 / 3}$, and $\lambda_{\mathrm{D}}^{2} \approx 2 \varepsilon E_{F} /\left(3 \mathrm{e}^{2} \mathrm{n}\right)$, which then gives

$$
\tau_{i i}^{\mathrm{deg}}=\frac{24 \pi^{3} \varepsilon^{2} \hbar^{3} n}{N_{I} Z^{2} e^{4} m_{p}^{*}\left[\ln \left(1+y_{F}\right)-y_{F} /\left(1+y_{F}\right)\right]}
$$

with $y_{F}=3^{1 / 3} 4 \pi^{8 / 3} \varepsilon \hbar^{2} \mathrm{n}^{1 / 3} /\left(\mathrm{m}_{\mathrm{p}}{ }^{*} \mathrm{e}^{2}\right)$. From Eq. 5.29, $\tau_{\mathrm{ii}}{ }^{\text {deg }}$ shows no explicit dependence on $\mathrm{T}$. However, the values $n$ and $N_{I}\left(N_{I}=n+2 N_{A}\right)$ have temperature dependences, though they may vary slowly.

By considering the aforementioned three intrinsic scattering mechanisms (i.e., po, pe and dp) and ii scattering, a fit to the measured Hall mobility can be performed. This fourmechanism approach will yield a fitting value for $\mathrm{N}_{\mathrm{a}}$. In practice, to get a best fit to measured data, especially at or above room temperature, $\mathrm{E}_{1}$ has been treated as a fitting parameter by many groups. For example, $\mathrm{E}_{1}$ values of $15 \mathrm{eV}$ (Look, 1998) and $31.4 \mathrm{eV}$ 
(Helbig, 1974) were used for $\mathrm{ZnO}$. A large $\mathrm{E}_{1}$ value would significantly lower $\mu_{\mathrm{dp}}$, and thus lower the intrinsic mobility curve $\mu_{\text {int }}$ from the predictions shown in Fig. 5.3. As a consequence, for example, the RT intrinsic mobility of $\mathrm{ZnO}$ would be well below 200 $\mathrm{cm}^{2} /$ Vs. In this work, we used the experimental $\mathrm{E}_{1}$ values. As a result, the intrinsic mobility of materials is not affected by the extrinsic factors such as impurity levels, hence a mobility limit for very pure samples could be established. On the other hand, the small $\mathrm{E}_{1}$ values associated with II-VI compounds require an additional scattering mechanism to achieve a good fit to the measured mobility, especially at higher temperatures. This additional mechanism, due to neutral impurities, will be discussed next.

The bound electron on a neutral impurity can also scatter a free electron passing by. Neutral impurity (ni) scattering is often ignored in mobility analysis of II-VI materials. If a measured RT mobility is well below the intrinsic prediction, the above four scattering mechanisms (i.e., dp, pe, po, and ii scatterings) are not sufficient to fit the measured mobility data. Scattering by neutral impurities is thus needed. As shown below, ni scattering has a weaker temperature dependence than ii scattering. At low temperatures, the effects of ni and ii scatterings on carrier mobility can have similar magnitudes. In some cases (e.g., hydrothermally grown $\mathrm{ZnO}$ ), it is the ni scattering that mainly accounts for the mobility decrease from the intrinsic prediction.

The relaxation time and mobility associated with ni scattering are (Erginsoy, 1950)

$$
\begin{aligned}
& \tau_{\mathrm{ni}}=\mathrm{m}_{\mathrm{p}}^{*} /\left(20 \mathrm{ha}_{0} \mathrm{~N}_{\mathrm{n}}\right)=\left(\mathrm{m}_{\mathrm{p}}^{*} \mathrm{e}\right)^{2} /\left(80 \pi \varepsilon \hbar^{3} \mathrm{~N}_{\mathrm{n}}\right) \\
& \mu_{\mathrm{ni}}=\mathrm{e} /\left(20 \mathrm{ha}_{0} \mathrm{~N}_{\mathrm{n}}\right)=\mathrm{m}_{\mathrm{p}} \mathrm{e}^{3} /\left(80 \pi \varepsilon \hbar^{3} \mathrm{~N}_{\mathrm{n}}\right)
\end{aligned}
$$

where $a_{0}$ is the effective Bohr radius of the donor and $N_{n}$ is the neutral impurity concentration given by $\mathrm{N}_{\mathrm{n}}=\mathrm{N}_{\mathrm{d}}-\mathrm{N}_{\mathrm{a}}-\mathrm{n}$. In this treatment, all the neutral scattering centers are treated as 
hydrogenic donors. If considering the existence of deep-level donors that have smaller $\mathrm{a}_{0}$, the fit of $N_{n}$ gives a lower limit of $N_{D}$ for the total neutral scattering centers.

By including po, pe, dp, ii and ni scatterings, a five-mechanism iterating approach can be applied to fit the measured Hall mobility data. Using $\mathrm{N}_{\mathrm{d}}$ and $\mathrm{N}_{\mathrm{a}}$ values obtained from an initial mobility fitting, $\tau_{\mathrm{ii}}$ and $\tau_{\mathrm{ni}}$, the relaxation times associated with ii and ni scatterings, respectively, were calculated, and the total Hall $\mathrm{r}$ factor $\mathrm{r}_{\mathrm{H}}(\mathrm{T})$ is found using $\tau_{\text {int }}$ and Matthiesen's rule. According to Erginsoy, $\tau_{\mathrm{ni}}$ is independent of $E$ and contributes a Hall $\mathrm{r}$ factor of exactly 1 . Thus, $\tau_{\mathrm{ii}}$ is the critical factor in determining the final shape of the $r_{H}(T)$

curve. In Eq. 5.27, although the $E^{3 / 2}$ dependence of $\tau_{\mathrm{ii}}$ is weakened by the energy-dependent term in the bracket of the denominator, $\tau_{\mathrm{ii}}$ still contributes a Hall $\mathrm{r}$ factor of about 1.6.

The calculated $\mathrm{r}_{\mathrm{H}}(\mathrm{T})$ was then used to correct the measured Hall mobility $\mu_{\mathrm{H}}(\mathrm{T})$ and Hall carrier concentration data $n_{H}(T)$, via equations $n(T)=n_{H}(T) * r_{H}(T)$ and $\mu(T)=\mu_{H}(T)$ $/ \mathrm{r}_{\mathrm{H}}(\mathrm{T})$. A second iteration of mobility fitting was then carried out with the modified $\mathrm{n}$ and $\mu$, which resulted in another set of fitting parameters $\mathrm{N}_{\mathrm{d}}$ and $\mathrm{N}_{\mathrm{a}} \cdot \mathrm{r}_{\mathrm{H}}(\mathrm{T})$ was re-calculated and $\mathrm{n}_{\mathrm{H}}$ and $\mu_{H}$ were modified again. A third iteration was sufficient to obtain final $\mathrm{N}_{\mathrm{d}}, \mathrm{N}_{\mathrm{a}}, \mathrm{r}_{\mathrm{H}}(\mathrm{T})$, and corrected $\mu(\mathrm{T})$ and $\mathrm{n}(\mathrm{T})$.

\subsection{Results and Discussion}

\subsubsection{Mobility Fitting of N-type ZnO}

In this work, Hall measurements and analyses were performed on four n-type $\mathrm{ZnO}$ samples which will be referred to here as EP, Cermet, TD and MTI. These samples were obtained from four different sources and grown by three different techniques. Sample EP was grown by the SCVT technique (Eagle-Picher Ind., Miami, OK). At $300 \mathrm{~K}$, the Hall 
carrier concentration and mobility measurements from sample EP are $\mathrm{n}_{\mathrm{H}}=9.0 \times 10^{16} \mathrm{~cm}^{-3}$ and $\mu_{\mathrm{H}}=205 \mathrm{~cm}^{2} /$ Vs. Sample Cermet was grown by a high-pressure melt (HPM) (Cermet, Atlanta, GA) and 300-K Hall results are $n_{H}=6.9 \times 10^{16} \mathrm{~cm}^{-3}$ and $\mu_{\mathrm{H}}=203 \mathrm{~cm}^{2} /$ Vs. Samples MTI (MTI Co., Richmond, CA) and TD (Tokyo Denpa, Japan) were grown by the hydrothermal (HYD) technique. For the MTI sample, $\mathrm{n}_{\mathrm{H}}=6.5 \times 10^{16} \mathrm{~cm}^{-3}$ and $\mu_{\mathrm{H}}=111$ $\mathrm{cm}^{2} /$ Vs at $300 \mathrm{~K}$. The TD sample gave $\mathrm{n}_{\mathrm{H}}=2.5 \times 10^{14} \mathrm{~cm}^{-3}$ and $\mu_{\mathrm{H}}=134 \mathrm{~cm}^{2} / \mathrm{Vs}$ at $300 \mathrm{~K}$. Temperature-dependent mobility measurements and fittings of the four $\mathrm{ZnO}$ samples are shown in Figure 5.5. For the EP (a) and Cermet (b) samples, low temperature mobilities are relatively high $\left(>1000 \mathrm{~cm}^{2} / \mathrm{Vs}\right)$. The fitting parameter $\mathrm{N}_{\mathrm{a}}$, which is associated with ionized impurity scattering, came out to be no more than $1 \times 10^{15} \mathrm{~cm}^{-3}$ for both of these samples. This makes $\mu_{\mathrm{ii}}$ insufficient to account for the total mobility drop as compared with the intrinsic limit at moderate to higher temperatures. Neutral impurity scattering amended this discrepancy. The total donor concentrations were found to be $\mathrm{N}_{\mathrm{d}}=2.7 \times 10^{17} \mathrm{~cm}^{-3}$ for sample EP and $1.7 \times 10^{17} \mathrm{~cm}^{-3}$ for sample Cermet.

For the moderate temperature range from 100 to $250 \mathrm{~K}$, the mobility fitting is still not satisfying for the EP and Cermet samples. The parameters used in Eqs. 5.9-12 and (5.20-21), e.g., $\mathrm{P}_{\perp}$ and $\mathrm{T}_{\mathrm{po}}$, are dependent on temperature. According to the temperature dependence of the dielectric piezoelectric stress elements and the permittivity elements (Kobiakov, 1980; Hill, 2003), the value of $P_{\perp}$ increases as temperature decreases. On the other hand, the value of $\mathrm{T}_{\mathrm{po}}$, should be smaller at lower temperature since lower-energy phonon modes will prevail. Ignoring the temperature dependence of these parameters may be the cause of the insufficiency in the mobility fitting. Meanwhile, there may be some other scattering mechanisms important in this temperature range. Dislocation scattering has been considered 


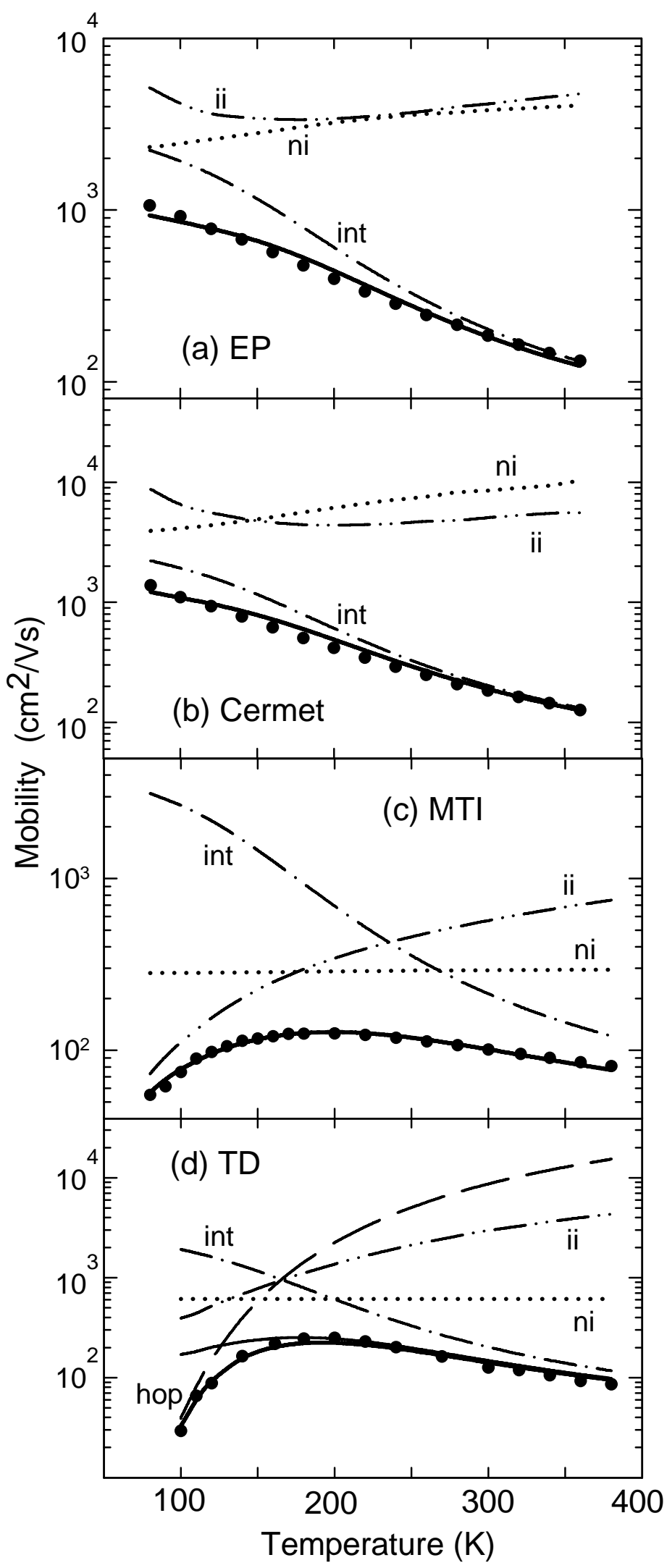

Figure 5.5. Teperaturedepentdent mobility data (solid circles) and fittings (solid lines) for four n-type $\mathrm{ZnO}$ samples. In the case of TD shown in (d), the thick solid line shows the fitting result after including the hopping effect (six-mechanism fitting), while the thinner solid line shows the result without the hopping effect (five-mechanism fitting). Notice the logarithmic vertical scale. 
as a candidate mechanism to improve the mobility fitting at moderate temperatures.

However, the temperature dependence of this mechanism did not help in the mobility fitting in either of the two samples discussed here, since (Ng,1998)

$$
\mu_{\text {dis }} \propto \mathrm{T}^{3 / 2} /\left(\mathrm{N}_{\mathrm{dis}} \lambda_{\mathrm{d}}\right)
$$

where $\lambda_{\mathrm{d}}$ is the Debye screening length as appeared in Eq. 5.26. The temperature dependence of $\mu_{\text {dis }}$ is opposite what is needed to improve the fitting. Non-polar optical phonon scattering is also considered. Its temperature dependence is described as

$$
\mu_{n p o}=\frac{\sqrt{2} \pi \rho e \hbar^{2}\left(\hbar \omega_{0}\right)^{1 / 2}}{D_{0}^{2}\left(m_{p}^{*}\right)^{5 / 2} n_{0}}
$$

where $\mathrm{n}_{0}=1 /\left[\exp \left(\hbar \omega_{0} / \mathrm{k}_{\mathrm{B}} \mathrm{T}\right)-1\right]$, and $\rho$ is the mass density. For $\mathrm{ZnO}$, the non-polar optical phonon energy is $\hbar \omega_{0} \approx 53 \mathrm{meV}$ (Alim, 2005) and $\mathrm{D}_{0}$, the optical phonon coupling constant, is found to be $1.0 \times 10^{11} \mathrm{eV} / \mathrm{m}$ following Hayashi et al. (Hayashi,1999). The temperature dependence of $\mu_{\mathrm{npo}}$ resembles that of $\mu_{\mathrm{po}}$, but its magnitude is about two orders higher than the latter in the whole temperature range concerned, thus can be ignored.

Some short-range interactions, such as space charge, alloy potential, and potential barrier scattering (Look, 1985), might play an important role in these two samples, because the mobility associated with these mechanisms has a temperature dependence of $\sim \mathrm{T}^{-1 / 2}$, which resembles the mobility behavior in the 'transition region' from impurity scattering to lattice scattering. However, lack of reliable parameters and the difficulty to evaluate the relative importance of these hard-to-distinguish effects prevent me from attacking them.

On the other hand, this five-scattering-mechanism mobility fitting approach works much better for the MTI sample (Fig. 5.5c). In this sample, the RT Hall measurement gave an $n_{H}$ value close to those from EP and Cermet samples, but the $\mu_{H}$ value was much lower. 
The Hall measurement gave a peak value of $\mu=130 \mathrm{~cm}^{2} / \mathrm{Vs}$ at about $170 \mathrm{~K}$. To fit the mobility data, the combination of $\mu_{\mathrm{ni}}$ and $\mu_{\mathrm{ii}}$ accounted for the low mobility for the entire temperature range measured. For the MTI sample, $\mathrm{N}_{\mathrm{d}}=2.2 \times 10^{18} \mathrm{~cm}^{-3}$ and $\mathrm{N}_{\mathrm{a}}=3.1 \times 10^{17}$ $\mathrm{cm}^{-3}$ were obtained from the fit. For all the three n-type samples discussed so far, $\mathrm{N}_{\mathrm{d}}$ is from one to two orders higher than $\mathrm{N}_{\mathrm{a}}$, while $\mu_{\mathrm{ni}}$ appears within the same order as $\mu_{\mathrm{ii}}$, although they undergo different temperature dependences.

In the TD sample, the mobility data show a peak of $210 \mathrm{~cm}^{2} / \mathrm{Vs}$ at about $220 \mathrm{~K}$. The five-mechanism fit was only good for $\mathrm{T} \geq 180 \mathrm{~K}$ (Fig. 5.5d, thin solid line), with fitting parameters $\mathrm{N}_{\mathrm{d}}=9 \times 10^{17} \mathrm{~cm}^{-3}$ and $\mathrm{N}_{\mathrm{a}}=5.1 \times 10^{16} \mathrm{~cm}^{-3}$. Below this temperature, the mobility drops very fast as temperature decreases in association with the fast freeze-out of free carriers. A sixth mechanism, the hopping mobility was then introduced to fit the low temperature mobility in this particular sample. An exponential equation was used for the hopping mobility as (Hirsch, 1979)

$$
\mu_{\text {hop }}=M_{\text {hop }} \exp \left(-E_{\text {hop }} / \mathrm{kT}\right)
$$

where $E_{\text {hop }}$ is the activation energy of hopping effect, and $M_{\text {hop }}$ is the high temperature limit of $\mu_{\text {hop. }}$ As shown in Figure 5.5(d) (thick solid line), the inclusion of hopping mobility gave a very good low-temperature mobility fit with a slight ease on ni and ii impurity scatterings $\left(\mathrm{N}_{\mathrm{d}}=7 \times 10^{17} \mathrm{~cm}^{-3}, \mathrm{~N}_{\mathrm{a}}=3.1 \times 10^{16} \mathrm{~cm}^{-3}\right)$, with $\mathrm{M}_{\text {hop }}=1.3 \times 10^{5} \mathrm{~cm}^{2} / \mathrm{Vs}$ and $\mathrm{E}_{\mathrm{hop}}=70 \mathrm{meV}$.

\subsubsection{Total Hall r Factor}

With all the intrinsic and extrinsic scattering mechanisms considered, the total Hall $r$ factor can be calculated and used to correct the measured mobility and free carrier concentration data. This can only be done in conjunction with the mobility fitting discussed 
above, because the parameters $\mathrm{N}_{\mathrm{a}}, \mathrm{N}_{\mathrm{d}}, \mathrm{M}_{\text {hop }}$, and $\mathrm{E}_{\text {hop }}$ have to be obtained from the initial fit. The calculated Hall $\mathrm{r}$ factor $\mathrm{r}_{\mathrm{H}}(\mathrm{T})$ curves for the four $\mathrm{ZnO}$ samples are shown in Figure 5.6 along with the predicted intrinsic curve $\mathrm{r}_{\mathrm{H}}{ }^{\mathrm{int}}(\mathrm{T})$ (solid line). For the EP and Cermet samples, $\mathrm{r}_{\mathrm{H}}(\mathrm{T})$ never exceeds 1.13 and the intrinsic Hall $\mathrm{r}$ factor curve, which is almost constant at 1.1, could be a good approximation for the whole temperature range. For the MTI sample, due to the relatively high acceptor concentration $\left(\mathrm{N}_{\mathrm{a}}\right)$, ionized impurity scattering is important at the lower temperatures and the Hall $r$ factor reaches maximum of $r_{\mathrm{H}} \approx 1.4$ at $80 \mathrm{~K}$. As temperature increases, ionized impurity scattering becomes less important compared with neutral impurity scattering. Also, as $\mathrm{T}$ increases, the intrinsic lattice scattering becomes dominant and therefore the total Hall $\mathrm{r}$ factor drops and reaches the intrinsic value 1.1. For the TD sample, the five-mechanism fit leads to a larger Hall-r factor and $\mathrm{r}_{\mathrm{H}} \approx 1.3$ at $100 \mathrm{~K}$. In the six-mechanism fit, since the relaxation time of the hopping effect has no dependence on the electron energy, it gives $\mathrm{r}_{\mathrm{hop}}=1$, and drops the total Hall $\mathrm{r}$ factor to below the intrinsic curve for the entire temperature range studied.

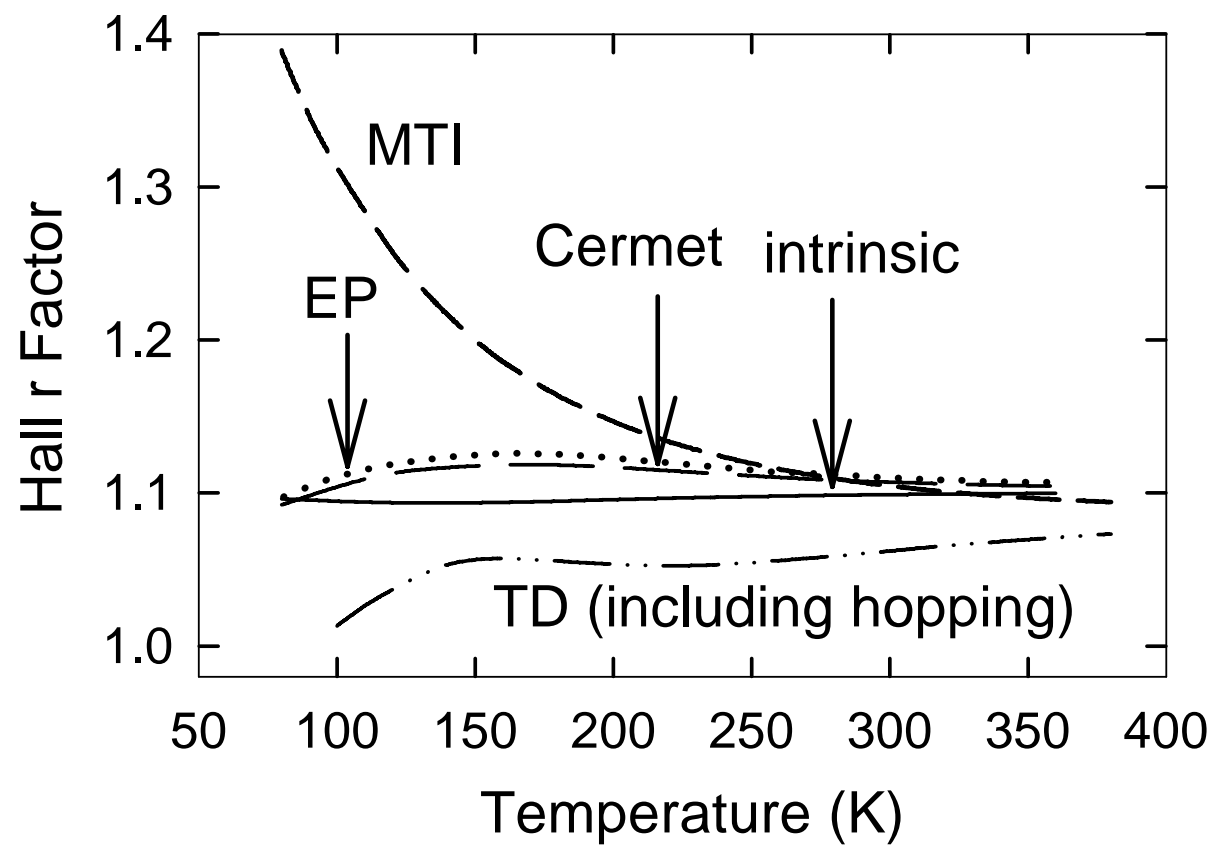

Figure 5.6. Temperaturedependent total Hall $r$ factor for four n-type $\mathrm{ZnO}$ samples. The intrinsic Hall $r$ factor curve is also shown (solid line) for comparison. 


\subsubsection{Free-carrier Concentration Fitting}

To fit the free carrier concentration data, the single donor model Eq. 5.3 was adopted with $\beta=1 / 2$ for samples EP, Cermet, and MTI. Figure 5.7 (a,b and c) shows the corrected $n$ data (open circles) and fitting results (solid lines) for these samples. The parameters obtained from the $n$-fitting are $\mathrm{N}_{\mathrm{D}}$ and $\mathrm{E}_{\mathrm{d}}$, with $\mathrm{N}_{\mathrm{a}}$ set at the value obtained from the $\mu$-fitting discussed above. I use $\mathrm{N}_{\mathrm{D}}$ here to distinguish this value from $\mathrm{N}_{\mathrm{d}}$ obtained from $\mu$-fitting, which are usually much different. Deep donor levels will contribute to ni scattering while they may have little effect on the electrical activation of free carriers. Table 5.1 presents these results along with $\mathrm{N}_{d}$ and $\mathrm{N}_{\mathrm{a}}$ from the $\mu$-fittings.

Table 5.1 Parameters obtained from mobility and free carrier concentration fittings for the four n-type $\mathrm{ZnO}$ samples.

\begin{tabular}{|c|c|c|c|c|c|c|c|}
\hline \multirow{2}{*}{ Sample } & \multicolumn{4}{|c|}{ Parameters obtained from $\mu$-fitting } & \multicolumn{3}{c|}{ Parameters from n-fitting } \\
\cline { 2 - 8 } & $\begin{array}{c}\mathbf{N}_{\mathbf{d}} \\
\left(\mathbf{c m}^{-3}\right)\end{array}$ & $\begin{array}{c}\mathbf{N}_{\mathbf{a}} \\
\left(\mathbf{c m}^{-3}\right)\end{array}$ & $\begin{array}{c}\mathbf{M}_{\mathbf{h o p}} \\
\left(\mathbf{c m}^{2} / \mathbf{V s}\right)\end{array}$ & $\begin{array}{c}\mathbf{E}_{\mathbf{a}} \\
(\mathbf{m e V})\end{array}$ & $\begin{array}{c}\mathbf{N}_{\mathbf{D}} \\
\left(\mathbf{c m}^{-3}\right)\end{array}$ & $\begin{array}{c}\mathbf{E}_{\mathbf{d}} \\
(\mathbf{m e V})\end{array}$ & $\begin{array}{c}\mathbf{E}_{\mathbf{d} \mathbf{0}} \\
(\mathbf{m e V})\end{array}$ \\
\hline EP & $2.7 \times 10^{17}$ & $1 \times 10^{15}$ & --- & --- & $1.8 \times 10^{17}$ & 35 & 62 \\
\hline Cermet & $1.7 \times 10^{15}$ & $1 \times 10^{15}$ & --- & --- & $1.1 \times 10^{17}$ & 49 & 72 \\
\hline MTI & $2.2 \times 10^{18}$ & $3.1 \times 10^{17}$ & --- & --- & $4.3 \times 10^{17}$ & 26 & 64 \\
\hline TD & $7 \times 10^{17}$ & $3.1 \times 10^{16}$ & $1.3 \times 10^{5}$ & 70 & $3.1 \times 10^{16}$ & 85 & 101 \\
\cline { 5 - 8 } & & & & & $8.5 \times 10^{16}$ & 263 & 285 \\
\hline
\end{tabular}

From the activation energy $E_{d}$ and donor concentration $N_{D}$, the ionization energy for dilute donor centers, $\mathrm{E}_{\mathrm{d} 0}$, could be estimated using Eq. 5.8. The donor ionization energy came out to be $62 \mathrm{meV}, 72 \mathrm{meV}$, and $64 \mathrm{meV}$ for the EP, Cermet, and MTI samples, respectively. By assuming a hydrogenic donor, the theoretical expectation of $\mathrm{E}_{\mathrm{d} 0}$ for $\mathrm{ZnO}$ 
would be $\mathrm{E}_{\mathrm{d} 0}=(13.6 \mathrm{eV})\left(\mathrm{m}_{\mathrm{p}}{ }^{*} / \mathrm{m}_{0}\right) / \mathrm{k}^{2}$. For $\mathrm{ZnO}$, with $\mathrm{m}_{\mathrm{p}}{ }^{*} / \mathrm{m}_{0}=0.29$, and $\mathrm{k}=7.8, \mathrm{E}_{\mathrm{d} 0}=65$ $\mathrm{meV}$ is predicted and is in good agreement with the experimental results of the three samples.

The compensated one-donor-level model Eq. 5.3 could not give a good fit for free carrier concentration data from the TD sample. The measured $300-\mathrm{K} \mathrm{n}_{\mathrm{H}}=2.5 \times 10^{14} \mathrm{~cm}^{-3}$, but from the mobility fitting $\mathrm{N}_{\mathrm{a}}=3.1 \times 10^{16} \mathrm{~cm}^{-3}$ was obtained. Thus, this sample is highly compensated. The fast freeze-out of free carriers suggests that the shallow donors are almost totally compensated by acceptors, and a deeper level of donor need to be activated to contribute to free electrons as temperature increases. A compensated two-donor-level model using Eq. 5.1 was then adopted to fit the $\mathrm{n}$ data from sample TD. The two-donor-level fitting for the TD sample had the best fit with $\beta_{1}=1 / 2$ and $\beta_{2}=1$ (Figure 5.7d). A shallow donor level with concentration $\mathrm{N}_{\mathrm{d} 1}=3.1 \times 10^{16} \mathrm{~cm}^{-3}$ and activation energy $\mathrm{E}_{\mathrm{d} 1}=85 \mathrm{meV}$ was found, along with a level having an activation energy $\mathrm{E}_{\mathrm{d} 2}=263 \mathrm{meV}$ with a much higher concentration, $\mathrm{N}_{\mathrm{d} 2}=8.5 \times 10^{16} \mathrm{~cm}^{-3}$, which gave an ionization energy $\mathrm{E}_{\mathrm{d} 20}=285 \mathrm{meV}$.

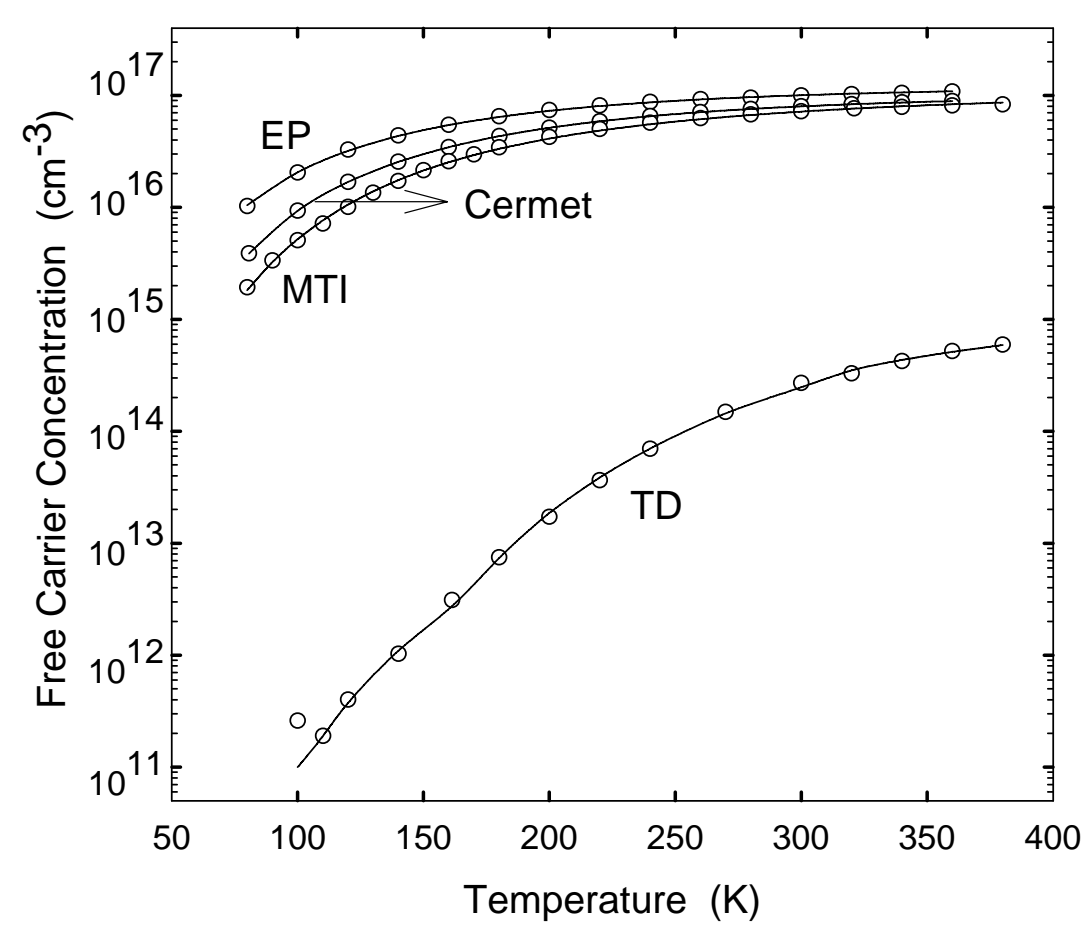

Figure 5.7. Free carrier concentration (n) fitting results for four n-type $\mathrm{ZnO}$ samples, using the onedonor-level model Eq. (5.3) for samples EP, Cermet and MTI, and the twodonor-level model Eq. (5.1) for sample TD. Notice the logarithmic vertical scale. 


\subsection{Identification of Impurities}

Liquid He temperature PL, FTIR, and EPR studies were used to identify the donors and acceptors revealed from Hall analysis in the preceding section. The 5-K PL spectra from the Cermet and MTI samples have intense donor-bound exciton $\left(\mathrm{D}^{0} \mathrm{X}\right)$ lines near $3.360 \mathrm{eV}$ (Fig. 5.8, black solid curve), indicating that the majority of the shallow donors in these samples are Group III donors, e.g., substitutional $\mathrm{Al}$ and $\mathrm{Ga}$. Using $\mathrm{E}_{\mathrm{x}}=3.377 \mathrm{eV}$ for $\mathrm{FX}$ transiton energy at $5 \mathrm{~K}$, the binding energies of these $\mathrm{D}^{0} \mathrm{X}$ lines were about $16-17 \mathrm{meV}$, corresponding to the hydrgenic donor binding energy. For the Cermet sample, a donor ionization energy of $71 \mathrm{meV}$ was obtained from the $\mathrm{B}^{0} \mathrm{X}$ binding energy and $\left(\mathrm{D}^{0}, \mathrm{~h}\right)$ band energy in Chapter 4, which is in very good agreement with the result obtained by Hall free carrier fitting $(72 \mathrm{meV})$ in the previous section. The dominant $\mathrm{D}^{0} \mathrm{X}$ line for the $\mathrm{EP}$ sample (Fig. 5.8, blue dashed line) is at $3.3625 \mathrm{eV}$, which is attributed to interstitial $\mathrm{H}$ in the literature (Meyer, 2004). The binding energy for this $\mathrm{D}^{0} \mathrm{X}$ line is $14.5 \mathrm{meV}$. From Hall free carrier concentration fitting, $\mathrm{E}_{\mathrm{D}}=62 \mathrm{meV}$ was found in the previous section for this sample.

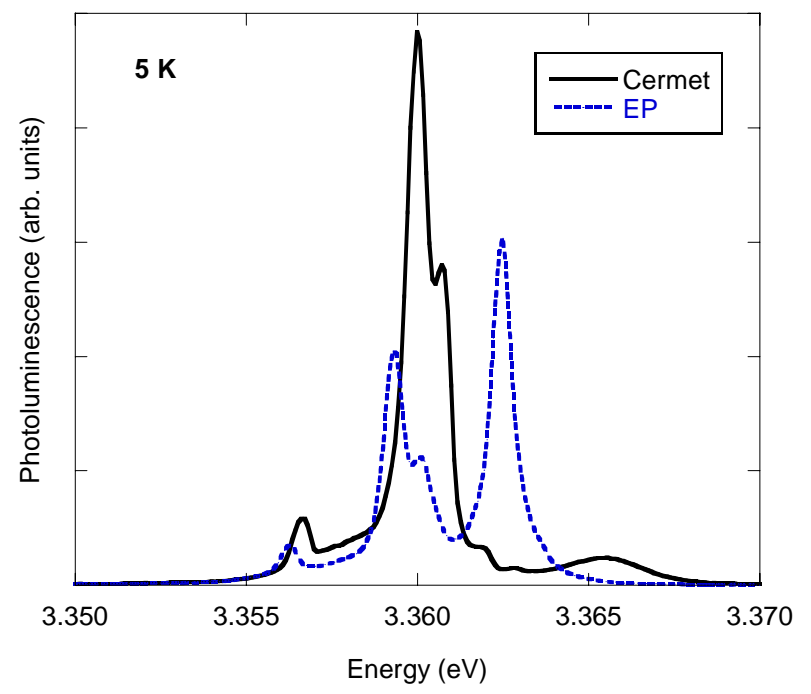

Figure 5.8. Photoluminescence (PL) edge emission showing sharp donorbound-exciton $\left(\mathrm{D}^{0} \mathrm{X}\right)$ lines from single crystal samples EP and Cermet.

The Haynes factor is $14.5 / 62=0.234$. This is consistent with the value obtained from $5-\mathrm{K}$

PL for the Cermet sample (0.235) in Chapter 4. From an EPR study on the HYD-grown TD 
sample, shallow donors in this sample are all ionized and are in their non-paramagnetic state. However, the typical shallow-donor signal at about $3450 \mathrm{G}$ could be observed after illuminating the sample with $442 \mathrm{~nm}$ Laser light, which puts the shallow donors into their neutral (e.g., paramagnetic) states. Also seen in this sample were the fingerprint signals of deep level substitutional transition metal ions $\mathrm{Mn}, \mathrm{Fe}, \mathrm{Ni}$, and $\mathrm{Co}$ in differing charge states (Giles, 2007). The Dominant deep donor in the TD sample at $\mathrm{E}_{\mathrm{d} 20}=285 \mathrm{meV}$ could be substitutional $\mathrm{Fe}^{2+}$ ions. The ionization of $\mathrm{Fe}^{2+}$ into $\mathrm{Fe}^{3+}$ could explain the $\beta$ value of 1 in the n-fitting of this sample, since both $\mathrm{Fe}^{2+}\left(3 \mathrm{~d}^{6}\right)$ and $\mathrm{Fe}^{3+}\left(3 \mathrm{~d}^{5}\right)$ have only one possible spin configuration. In another EPR study of the TD sample, the activation energy for $\mathrm{Fe}^{2+}$ was estimated to be about $240 \mathrm{meV}$ ( $\pm 20 \%$ uncertainty) through a pulsed thermal-anneal approach (Jiang, 2007). Due to the hopping effect, the activation energy of the shallower level is not reflecting the real donor level, and cannot be clearly assigned to a certain defect. A multiphonon hopping model could reproduce the conductivity data for $\mathrm{T} \leq 200 \mathrm{~K}$ using (Look, 1996)

$$
\sigma(\mathrm{T})=\sigma_{0} \exp \left(-\epsilon_{3} / \mathrm{k}_{\mathrm{B}} \mathrm{T}\right] \exp \left[-\left(\mathrm{T}_{0} / \mathrm{T}\right)^{1 / 4}\right]
$$

with activation energy $\epsilon_{3}=6$ mev and $T_{0}=3 \times 10^{8} \mathrm{~K} . \mathrm{T}_{0}$ and $\epsilon_{3}$ are related as

$$
\mathrm{k}_{\mathrm{B}} \mathrm{T}_{0}=21.3 / \mathrm{g}\left(\epsilon_{\mathrm{F}}\right) \mathrm{a}_{\mathrm{d}}{ }^{3}
$$

where $\mathrm{g}\left(\epsilon_{\mathrm{F}}\right) \approx \mathrm{N}_{\mathrm{a}} / \epsilon_{3}$ is the density of states in the donor band, and $\mathrm{a}_{\mathrm{d}}$ is a characteristic length representing the extent of the wavefunction of the defect level involved in the hopping process. The activation energy is expected to be $\epsilon_{3} \approx \mathrm{e}^{2} \mathrm{~N}_{\mathrm{D}}^{1 / 3} /\left(4 \pi \mathrm{k} \varepsilon_{0}\right)$. With $\mathrm{ND}=$ $3.1 \times 10^{16} \mathrm{~cm}^{-3}$ from the free carrier fitting, $\epsilon_{3} \approx 5.8 \mathrm{meV}$ was predicted. Using $\epsilon_{3}=6 \mathrm{mev}$ and $\mathrm{T}_{0}=3 \times 10^{8} \mathrm{~K}$ obtained from conductivity fitting, $\mathrm{a}_{\mathrm{d}}=5.4 \AA$ was found. Considering the range of $\mathrm{T}_{0}$ from fittings, e.g., $\mathrm{T}_{0}=1.5-5.0 \times 10^{8} \mathrm{~K}, \mathrm{a}_{\mathrm{d}}$ varies within the range of $5 \sim 7 \AA$. The 
values $T_{0}$ and $a_{d}$ obtained for the TD sample in this study are close to those reported for a GaN sample where hopping effect affects the conductivity and mobility behavior for a temperature range of 80-200K (Look, 1996).

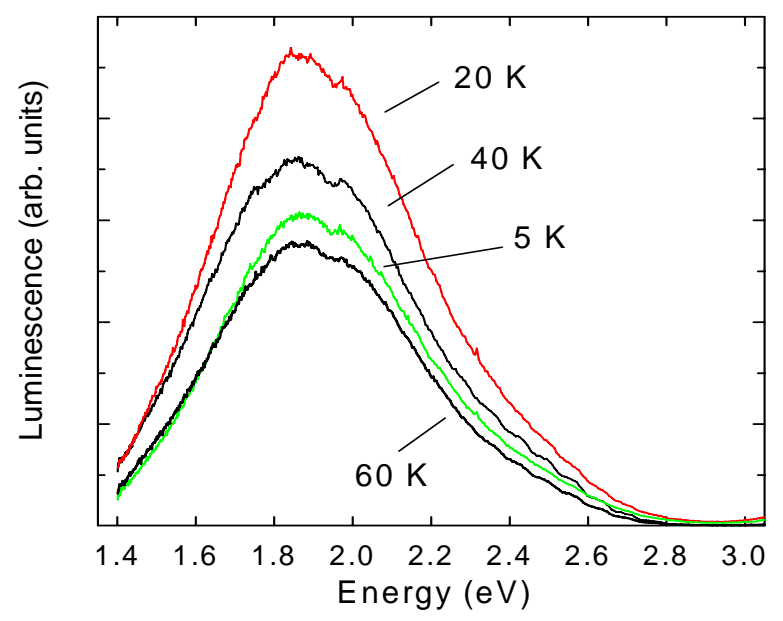

Figure 5.9. Donor-acceptor-pair (DAP) PL emission from sample TD. This peak involves a shallow donor and a neutral $\mathrm{Li}$ acceptor (from Giles, 2007).

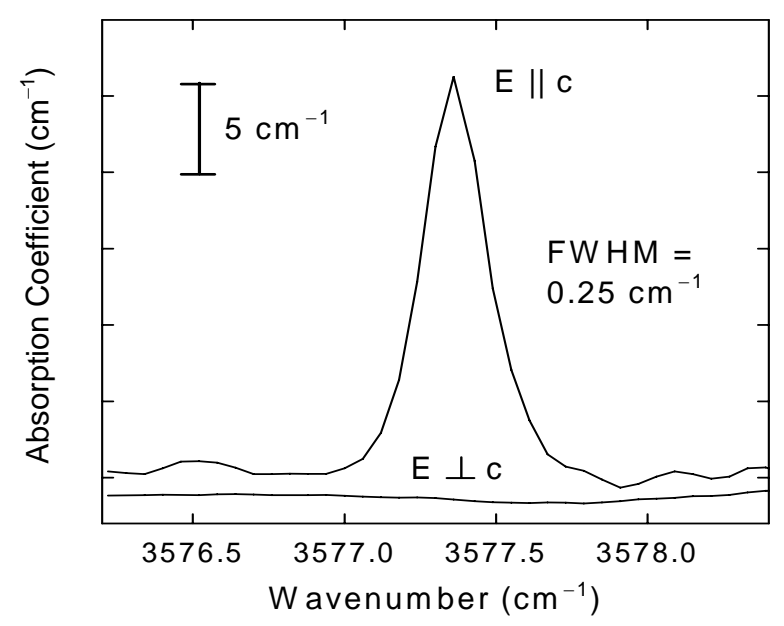

Figure 5. 10. The LI-OH ${ }^{-}$absorption peak observed at $10 \mathrm{~K}$ from a HYD grown sample TD. The peak can only be seen with the light polarization parallel to the c-axis of the sample. (Giles, 2007).

The relatively high concentrations of acceptors found in the two HYD samples, MTI and TD, must have come from the mineralizer used in the growth technique. EPR spectra revealed isolate neutral Li acceptors in the TD samples (Giles, 2007). 5-K PL from this sample has a yellow donor-acceptor-pair (DAP) peak which involves a neutral Li acceptor and a shallow donor (Fig. 5.19) (Giles, 2007). However, most of the lithium impurities in these HYD samples exist in the form of $\mathrm{LiOH}^{-}$complex, which was observed in FTIR 
spectra from both samples at $3577 \mathrm{~cm}^{-1}$. Figure 5.10 shows the $\mathrm{LiOH}^{-}$absorption line observed from the TD sample at $10 \mathrm{~K}$, which has a FWHM of $0.25 \mathrm{~cm}^{-1}$ (Giles, 2007). This line could only be observed at near liquid He temperature and with light polarization parallel to the c-axis of the sample (Halliburton, 2004). Li in this complex form are not electronically active and cannot be observed in EPR or PL spectra, but it can serve as neutral scattering centers. From the intensity of the peak at $3577 \mathrm{~cm}^{-1}$ in FTIR spectra, the concentration of $\mathrm{LiOH}^{-}$complexes in the MTI sample was estimated to be about $3 \times 10^{17} \mathrm{~cm}^{-3}$. In the TD sample, the concentration of $\mathrm{LiOH}^{-}$complexes was about $8 \times 10^{17} \mathrm{~cm}^{-3}$, which could account for the total neutral center density $\mathrm{N}_{\mathrm{d}}$ revealed from the mobility fitting.

\subsection{Hole Mobility in P-type ZnO}

As-grown $\mathrm{ZnO}$ samples always intend to be n-type, with typical donor concentrations of $10^{16}$ to $10^{18} \mathrm{~cm}^{-3}$. To realize p-type conductivity, one has to intentionally dope with acceptors with a density high enough to compensate the donors. In this work, I applied the three intrinsic scattering mechanisms to p-type $\mathrm{ZnO}$ material. I used an effective hole polaron mass $\mathrm{m}_{\mathrm{p}}{ }^{*}=0.59 \mathrm{~m}_{0}$ and found the $300-\mathrm{K}$ intrinsic hole mobility to be about 80 $\mathrm{cm}^{2} /$ Vs. Then, by assuming specific values for the densities of shallow donors (native or incorporated) $\left(\mathrm{N}_{\mathrm{d}}\right)$, and intentionally doped acceptors $\left(\mathrm{N}_{\mathrm{a}}\right)$, I was able to incorporate the contributions from ionized and neutral impurity scatterings and simulate the temperaturedependent mobility behavior of a p-type doped $\mathrm{ZnO}$ sample. To compare the simulation results with reported measurements, I adopted the acceptor activation energy $\left(E_{a}\right)$ values as extrapolated by several groups from arsenic-doped $\mathrm{ZnO}$, which will give the temperature dependent hole concentration (p). For example, $\mathrm{E}_{\mathrm{a}}=120 \mathrm{meV}$ (Ryu, 2003) and $90 \mathrm{eV}$ (Look, 
2004) have been reported. By assuming a "hydrogenic" model for acceptors, one gets an ionization energy of $(13.6)(0.59) / 7.82=0.132 \mathrm{eV}=132 \mathrm{meV}$. The relaxation time equations related to different scattering mechanisms were the same as used in n-type cases except for necessary substitutions, such as hole polaron mass, $\mathrm{m}_{\mathrm{p}}{ }^{*}$, the ionized impurity density, $\mathrm{N}_{\mathrm{i}}=\mathrm{p}$ $+2 \mathrm{~N}_{\mathrm{d}}$, and the neutral impurity density, $\mathrm{N}_{\mathrm{n}}=\mathrm{N}_{\mathrm{a}}-\mathrm{N}_{\mathrm{d}}-$ p. The charge balance Eq. 5.3 was used to generate free hole concentration based on a single acceptor level $\mathrm{E}_{\mathrm{a}}$ with a concentration $\mathrm{N}_{\mathrm{a}}$ and partially compensated by a donor concentration of $\mathrm{N}_{\mathrm{d}}$.

A RT hole mobility of about $35 \mathrm{~cm}^{2} / \mathrm{Vs}$ with $\mathrm{p}=4 \times 10^{17} \mathrm{~cm}^{-3}$ has been reported by Ryu et al. from arsenic-doped p-type $\mathrm{ZnO}$ samples (Ryu, 2003). From free carrier analysis, they got $\mathrm{N}_{\mathrm{a}}=\mathrm{N}_{\mathrm{As}}=3 \times 10^{18} \mathrm{~cm}^{-3}$, and extrapolated a thermal binding energy $\mathrm{E}_{\mathrm{A}}{ }^{\text {th-b }}=120$ meV using the relation $\mathrm{p} \sim \mathrm{T}^{3 / 2} \exp \left(-\mathrm{E}_{\mathrm{a}}^{\text {th-b }} / \mathrm{k}_{\mathrm{B}} \mathrm{T}\right)$. A $300-\mathrm{K}$ mobility of about $5 \mathrm{~cm}^{2} / \mathrm{Vs}$ with $\mathrm{p}$ $\approx 4 \times 10^{18} \mathrm{~cm}^{-3}$ was reported for rf-sputtered, P-type ZnO:As (Look, 2004). Defect concentrations of $\mathrm{N}_{\mathrm{d}}=8 \times 10^{19} \mathrm{~cm}^{-3}$ and $\mathrm{N}_{\mathrm{a}}=8 \times 10^{19} \mathrm{~cm}^{-3}$ were obtained from mobility and hole concentration fitting, with an extrapolated acceptor activation energy of $E_{a}=90 \mathrm{meV}$.

Figure 5.11 shows one of the simulation results of $\mathrm{p}$-type doped $\mathrm{ZnO}$ for the temperature range $60-400 \mathrm{~K}$, using the five-mechanism approach. The intrinsic mobility curve is shown along with the impurity scattering mobilities. To generate the temperature dependent free hole concentration $\mathrm{p}$, I assumed an acceptor concentration of $\mathrm{N}_{\mathrm{a}}=3.0 \times 10^{18}$ $\mathrm{cm}^{-3}$ with an activation energy $\mathrm{E}_{\mathrm{a}}=120 \mathrm{meV}$ (Ryu, 2003). By selecting a compensating donor concentration of $\mathrm{N}_{\mathrm{d}}=1.7 \times 10^{18} \mathrm{~cm}^{-3}$, the simulation result shows that the total hole mobility curve has a peak of $31 \mathrm{~cm}^{2} / \mathrm{Vs}$ at $300 \mathrm{~K}$, corresponding to a Hall mobility of about $35 \mathrm{~cm}^{2} /$ Vs. Thus, the measured mobility value in the thin-film work could be simulated, 
however, the $300-\mathrm{K}$ Hall hole concentration was found to be $\mathrm{p}=2.4 \times 10^{16} \mathrm{~cm}^{-3}$, much smaller than the reported value of $4 \times 10^{17} \mathrm{~cm}^{-3}$.

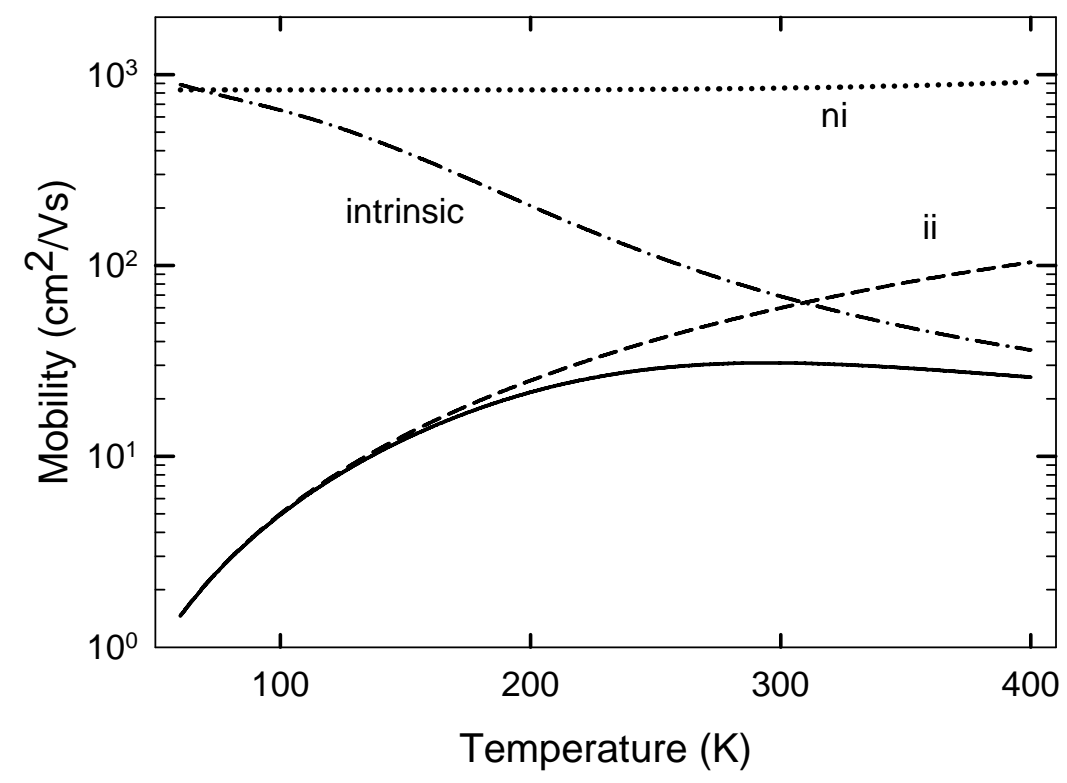

Figure 5.11. Simulation of hole mobility (solid line) in a p-type sample having $\mathrm{N}_{\mathrm{a}}=3.0 \times 10^{18} \mathrm{~cm}^{-3}$, and $\mathrm{N}_{\mathrm{d}}=1.7 \times 10^{18} \mathrm{~cm}^{-3}$. This case corresponds to a 300 $\mathrm{K}$ Hall mobility $=35$ $\mathrm{cm}^{2} / \mathrm{Vs}$.

In the simulation above, the compensation rate $\theta=\mathrm{N}_{\mathrm{d}} / \mathrm{N}_{\mathrm{a}} \approx 0.6$ and a spin-degeneracy ratio $\beta=1 / 2$ were used. In fact, as discussed in the beginning of this section, $\mathrm{p}$-type $\mathrm{ZnO}$ samples usually have high compensation rates, $\mathrm{N}_{\mathrm{a}} \approx \mathrm{N}_{\mathrm{d}}>\mathrm{p}$, and the total ionized impurity concentration $\mathrm{N}_{\mathrm{i}}=\mathrm{p}+2 \mathrm{~N}_{\mathrm{d}} \approx 2 \mathrm{~N}_{\mathrm{d}}$, and total neutral impurity concentration $\mathrm{N}_{\mathrm{n}}=\mathrm{N}_{\mathrm{a}}-\mathrm{N}_{\mathrm{d}}-\mathrm{p}$ $\approx \mathrm{N}_{\mathrm{a}}-\mathrm{N}_{\mathrm{d}}$ can be very large. For a given value of $\mathrm{N}_{\mathrm{a}}$, a higher compensation makes $\mu_{\mathrm{ii}}$ more important and $\mu_{\mathrm{ni}}$ less important, which changes the shape of the total mobility curve and moves the peak value to higher temperatures. On the other hand, the charge balance equation for $p$-type material, $p\left(p+N_{d}\right) /\left(N_{a}-N_{d}-p\right)=\beta N_{v} \exp \left(-E_{a} / k_{B} T\right)$, can be simplified as $p \approx$ $\left(\mathrm{N}_{\mathrm{a}} / \mathrm{N}_{\mathrm{d}}-1\right) \beta \mathrm{N}_{\mathrm{v}} \exp \left(-\mathrm{E}_{\mathrm{a}} / \mathrm{k}_{\mathrm{B}} \mathrm{T}\right)=(1 / \theta-1) \beta \mathrm{N}_{\mathrm{v}} \exp \left(-\mathrm{E}_{\mathrm{a}} / \mathrm{k}_{\mathrm{B}} \mathrm{T}\right)$. As $\theta$ reaches unity, $\mathrm{p}$ becomes very small as compared to the total number of available states in the valence band.

The choice of the $\beta$ value for $\mathrm{p}$-type $\mathrm{ZnO}$ is debatable. If one ignores the energy splitting between the $\mathrm{A}$ and $\mathrm{B}$ valence bands in $\mathrm{ZnO}$, and treats them as two-fold degenerate, 
as for the zincblende materials, $\beta=1 / 4$ should be used. In fact, $\beta=1 / 4$ has been adopted in literature for p-type (Look, 2002) However, if one recognizes the small energy splitting of these two valence bands and treats them as non-degenerate, then $\beta=1 / 2$ is a proper choice since only the top band is considered. Figure 5.12 shows the $300-\mathrm{K}$ hole mobility simulation results as a function of compensation rate $\theta$. In this simulation example, I used $\mathrm{N}_{\mathrm{a}}=3.0 \mathrm{x}$ $10^{18} \mathrm{~cm}^{-3}$ and $E_{a}=120 \mathrm{meV}$. The effects of $\beta=1 / 4$ and $\beta=1 / 2$ are compared.

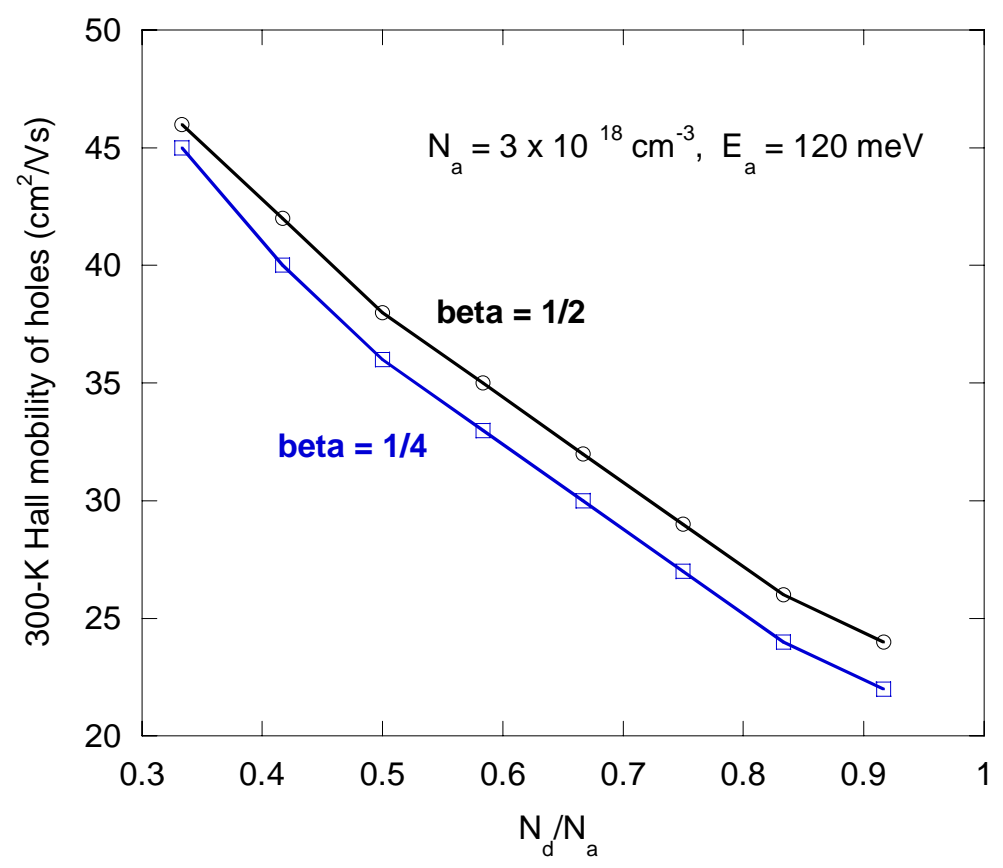

Figure 5.12. Simulated Hall mobility of holes at $300 \mathrm{~K}$ as a function of compensation rate $\theta=\mathrm{N}_{\mathrm{d}} / \mathrm{N}_{\mathrm{a}}$ with given parameters beta, $\mathrm{N}_{\mathrm{a}}$, and $\mathrm{E}_{\mathrm{a}}$.

Figure 5.13 shows another simulation result where the p-type material is assumed to be heavily doped and highly compensated, with $\mathrm{N}_{\mathrm{a}}=9 \times 10^{19} \mathrm{~cm}^{-3}, \mathrm{~N}_{\mathrm{d}}=8 \times 10^{19} \mathrm{~cm}^{-3}$, and activation energy $E_{a}=90 \mathrm{meV}$ being used, as reported by Look (Look, 2004). Since the donors are assumed to be all ionized, $\mu_{\mathrm{ii}}$ dominates the total mobility for the whole temperature range. In this situation, the choice of $\beta=1 / 2$ or $\beta=1 / 4$ does not affect the mobility because in both cases the free carriers are found to be only in the low-10 $16 \mathrm{~cm}^{-3}$ range, totally negligible in the expressions of $\mathrm{N}_{\mathrm{i}}$ and $\mathrm{N}_{\mathrm{n}}$. At $300 \mathrm{~K}$, the total Hall hole 
mobility is only $\sim 2 \mathrm{~cm}^{2} / \mathrm{Vs}$. As temperature decreases, mobility drops quickly and is predicted to be less than $0.1 \mathrm{~cm}^{2} / \mathrm{Vs}$ at $60 \mathrm{~K}$.

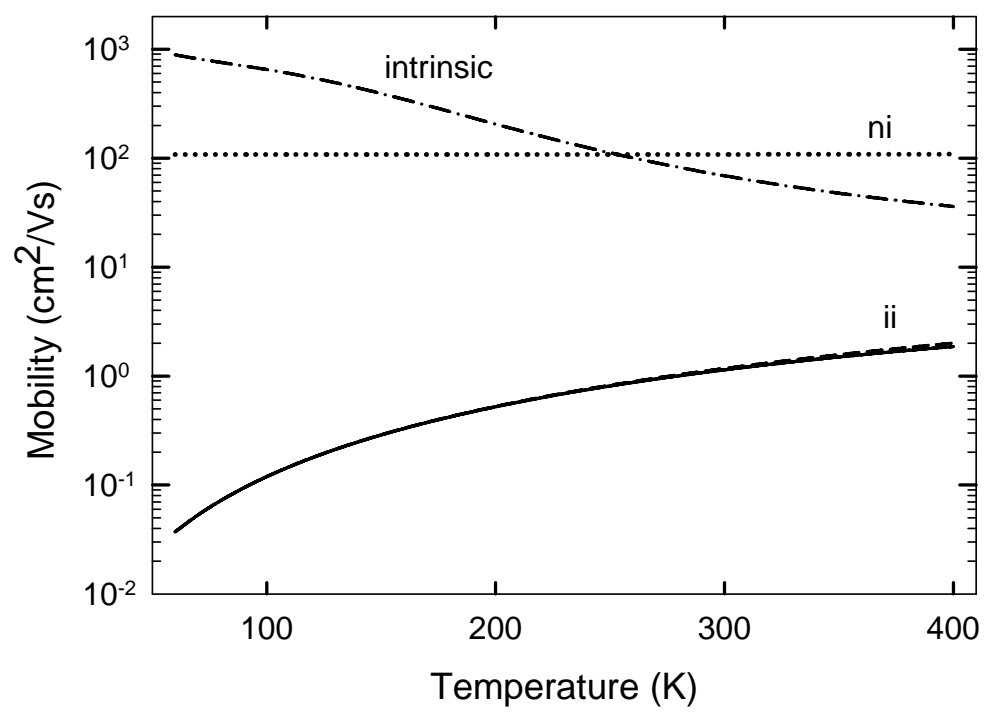

Figure 5.13. Simulation of hole mobility in a p-type sample doped with $\mathrm{N}_{\mathrm{a}}=9.0 \times 10^{19} \mathrm{~cm}^{-3}$, and compensated by $\mathrm{N}_{\mathrm{d}}=8.0 \mathrm{x}$ $10^{19} \mathrm{~cm}^{-3} \cdot 300 \mathrm{~K}$ Hall mobility $=2 \mathrm{~cm}^{2} / \mathrm{Vs}$.

In summary, using the relaxation time approximation approach, the intrinsic electron mobility limit for $\mathrm{n}$-type $\mathrm{ZnO}$ is established, and used to fit measured temperature dependent Hall mobility data by including both ionized and neutral impurity scattering. Hall $\mathrm{r}$ factor was calculated used to correct measured mobility and free carrier concentration data. Similar approach was applied in prediction of hole mobility in n-type $\mathrm{ZnO}$. At $300 \mathrm{~K}$, the predicted intrinsic electron and hole Hall mobilities are about 220 and $80 \mathrm{~cm}^{2} / \mathrm{Vs}$, respectively. 


\section{Chapter 6 Effects of Doping and Post-growth Treatments on Optical Properties}

\section{of Single Crystal ZnO}

In Chapter 1, PL emissions from a heavily doped n-type ZnO:Ga sample was compared with an as-grown sample from the same growth technique. The $\mathrm{ZnO}$ :Ga sample has a RT PL 4 times brighter than the undoped sample. In search of brighter UV scintillators, highly n-type $\mathrm{ZnO}$ samples were prepared by either doping with extrinsic dopants, or annealing in different atmospheres to introduce native defects. In this chapter, I present the optical absorption study on the effects of such treatments.

Single crystal $\mathrm{ZnO}$ samples were grown from three different growth techniques: seeded chemical vapor transport (SCVT), high-pressure melt (HPM), and hydrothermal (HYD). Most of the as-grown samples are clear in color, while some are light yellow. Intentional doping, contamination, or after-growth treatments can change the color of samples. The color change in samples can be related to their optical absorption spectra, which affects the luminescence properties of the samples.

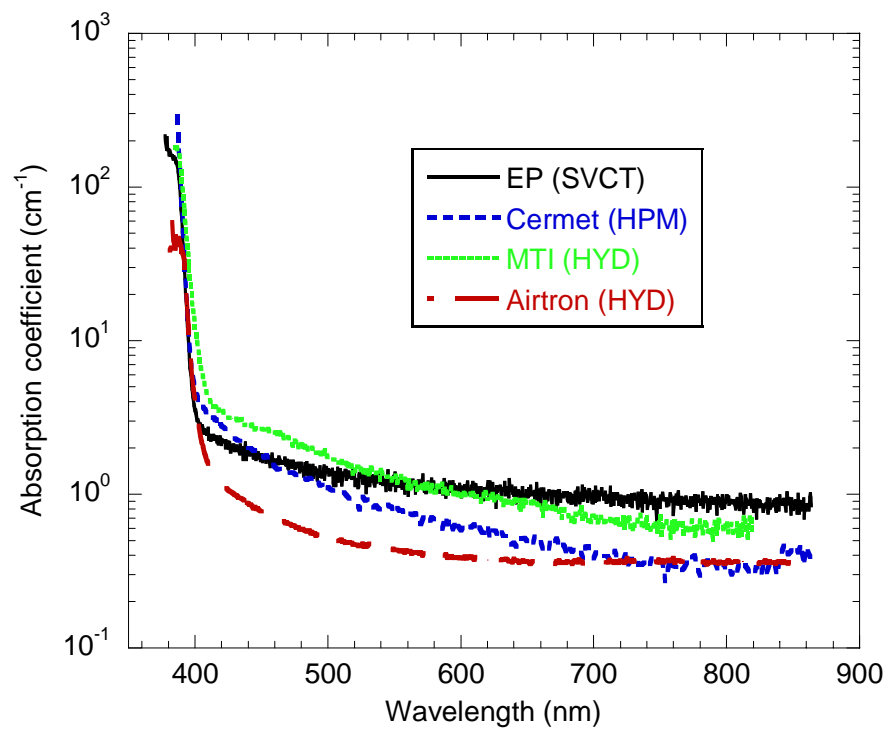

Figure 6.1 Absorption coefficient from four as-grown single crystal samples grown from seeded chemical vapor transport (SCVT), highpressure melt (HPM), and hydrothermal (HYD) techniques. Note the vertical logarithmic scale. 
Figure 6.1 shows the absorption spectra of four as grown single crystal samples from the above growth techniques. The EP, Cermet, and MTI samples are grown by SCVT, HPM, and HYD techniques, respectively, as have been described in Chapter 5. The Airtron sample was HYD-grown (Airtron, NJ) and its thickness is $1.9 \mathrm{~mm}$. It has a RT free carrier concentration of $2 \times 10^{13} \mathrm{~cm}^{-3}$, and is semi-insulate. As shown in Fig. 6.1, all four samples have an absorption edge at about $390 \mathrm{~nm}(3.18 \mathrm{eV})$, and they are highly transparent for the whole visible range $(400-800 \mathrm{~nm})$. The MTI sample has an absorption peak in the range $420-500 \mathrm{~nm}$, which is so weak that the sample still looks clear, as the rest samples do.

\subsection{Effects of Heavy Doping on $\mathrm{ZnO}$}

Doped $\mathrm{ZnO}$ single crystals were grown at Cermet, Inc (Atlanta, GA) by HPM technique, using dopant elements including group III (Ga, In), group II (Mg), group I (Li), and lanthanides (Gd, Er, Tm). Co-doping was performed with Ga-Li, In-Li, Er-Li and MgGa combinations. Sample colors vary from clear, light green, green, to dark blue (Neal, 2008). The $\mathrm{ZnO}$ :Ga sample discussed in Ch. 4 has a light blue color. The room temperature Hall measurement gave $\mathrm{n}=2 \times 10^{19} \mathrm{~cm}^{-3}$. Using the same growth technique Ga and Li codoping was conducted and resulted in dark blue crystals. Figure 6.2 shows the absorption coefficient of four $\mathrm{ZnO}: \mathrm{Ga}, \mathrm{Li}$ samples along with the $\mathrm{ZnO}: \mathrm{Ga}$ sample and an as-grown Cermet sample. The short-wavelength tail of free-carrier absorption spectrum extends to about $450 \mathrm{~nm}$ for the four $\mathrm{Ga}-\mathrm{Li}$ co-doped samples, leaving a very narrow transparent window in the 420-500 range. This is the origin of the dark-blue color of these samples, and the light blue color in the $\mathrm{ZnO}$ :Ga sample. The four $\mathrm{ZnO}$ :Ga,Li samples shown in Fig. 6.2

have dimensions of 10x10x4 $\mathrm{mm}^{3}$, and a Hall measurement cannot be performed using the 
Hall system described in Chapter 3. The absorption data are therefore used to obtain the RT free carrier concentration for these samples. Using the Drude model for free electrons, the

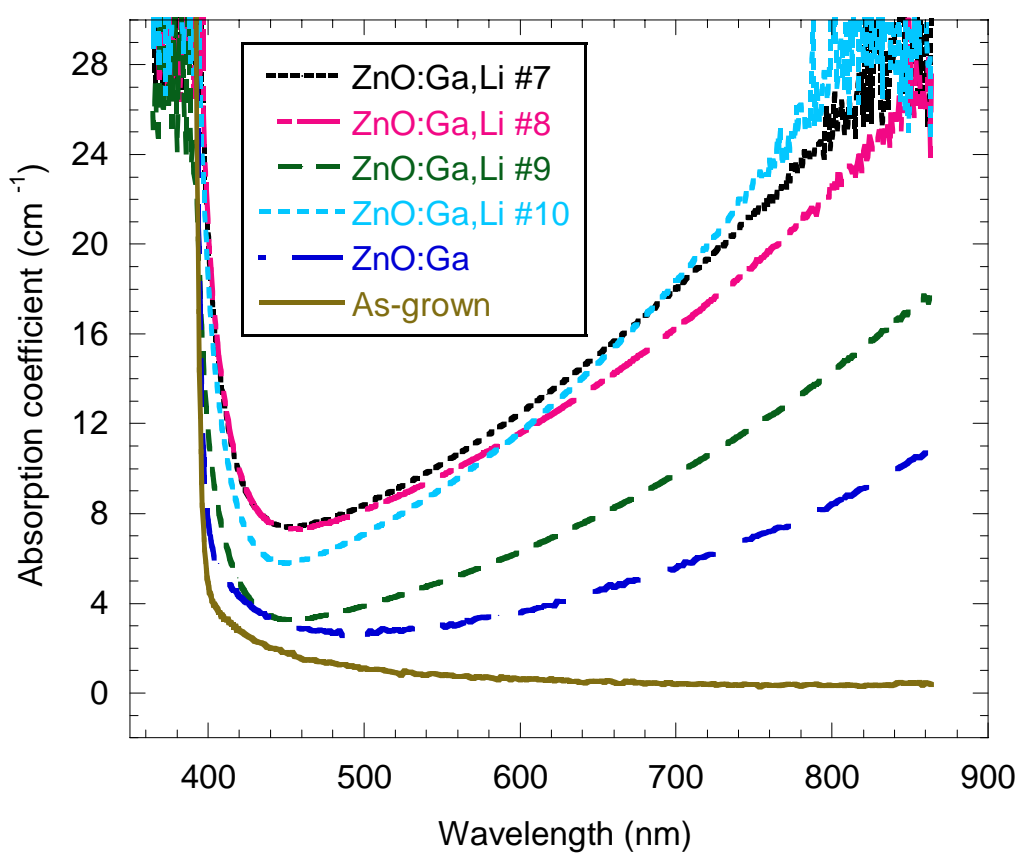

Figure 6.2 Absorption spectra of four $\mathrm{ZnO}$ single crystal samples codoped with $\mathrm{Ga}$ and Li compared with the $\mathrm{ZnO}: \mathrm{Ga}$ sample and an as-grown sample. All samples were grown by HPM technique at Cermet Inc. (Atlanta, GA).

effect of free carriers on the sample absorption coefficient $(\alpha)$ is described as (Fox, 2001)

$$
\alpha=\frac{N e^{2}}{n \varepsilon_{0} c m_{e}^{*} \tau} \frac{1}{\omega^{2}}
$$

where $\mathrm{N}$ is free carrier concentration, $\mathrm{n}$ is refractive index, $\varepsilon_{0}$ is the static permittivity, $\tau$ is the free-carrier scattering relaxation time, and $\omega$ is the frequency of absorption $(\omega \sim 1 / \lambda)$. If one assumes the wavelength dependence for relaxation time as $\tau \sim \lambda^{\mathrm{q}}$, where $\mathrm{q}$ is a constant depending on what scattering mechanism is the dominant contributor to $\tau$, then Eq. 6.1 predicts an absorption depending on $\mathrm{N}$ and $\lambda$ according to $\alpha \sim N \lambda^{\mathrm{p}}$, with $\mathrm{p}=2$-q. This relation was used to estimate the free carrier concentrations in the $\mathrm{ZnO}: \mathrm{Ga}, \mathrm{Li}$ samples shown in Fig. 6.2. At a wavelength of $\lambda=700 \mathrm{~nm}$, the absorption coefficients for the four co-doped samples were compared with that of the $\mathrm{ZnO}$ :Ga sample. Since the $\mathrm{n}$ value for $\mathrm{ZnO}: \mathrm{Ga}$ was 
known from a Hall effect measurement, the $\mathrm{n}$ values for the other samples could be determined if similar scattering processes were assumed. The results are shown in Table 6.1. The four dark-blue ZnO:Ga,Li codoped samples shown in Fig. 6.2 are higher n-type than the ZnO:Ga sample. However, RT PL under the same excitation conditions show that these $\mathrm{ZnO}: \mathrm{Ga}, \mathrm{Li}$ samples are less bright than the $\mathrm{ZnO}$ :Ga sample. However, two samples that were grown by the same technique and codoped with Er-Li and In-Li, respectively, were found $1.5 \sim 2$ times brighter than the $\mathrm{ZnO}: \mathrm{Ga}$. Using the absorption approach, the free carrier concentrations were found to be $8.4 \times 10^{18}$ and $8.5 \times 10^{18} \mathrm{~cm}^{-3}$ for these two samples. So, although high n-type is desired for $\mathrm{ZnO}$ scintillators, it not simply true that the higher $\mathrm{n}$, the brighter UV emission. An optimum carrier concentration may exist below the Mott density.

The value of the exponent $p$ was obtained by fitting the absorption curve with a power law regression. If the absorption data in Figure 6.2 are plotted in logarithmic scale for both axes, as shown in Figure 6.3, the power-law relation is indicated by the straight lines. The $\mathrm{p}$ values found from the power-law fit of the five doped $\mathrm{ZnO}$ samples are listed in Table 6.1. The range in $\mathrm{p}$ for the Ga-Li co-doped samples is from 2.1 to 2.9 , and the $\mathrm{ZnO}: \mathrm{Ga}$ sample was described by $p=3$. As discussed in Chapter 5, different electron scattering mechanisms have different energy dependences, giving different $q$ values in the expression $\tau$ $\sim \lambda^{\mathrm{q}}$. Intrinsic phonon scattering processes will give a $\mathrm{q}$ value of about $-1 / 2$ at room temperature, thus $\mathrm{p}=2.5$ is expected for a very pure sample. For these heavily doped samples, the ionized impurity scattering could be a dominant contributor to $\tau$ even at room temperature, giving a q value of $-3 / 2$, and $p$ value of 3.5 is expected. The measured values of $\mathrm{p}$ for these samples are in good agreement with this range. 
Table 6.1 Free-carrier concentration and the p-value obtained from absorption data.

\begin{tabular}{|c|c|c|c|}
\hline Sample & $\boldsymbol{\alpha}$ at $\left.\mathbf{7 0 0} \mathbf{~ n m ~} \mathbf{( c m}^{\mathbf{- 1}}\right)$ & $\mathbf{N}\left(\mathbf{c m}^{\mathbf{3}}\right)$ & p value in $\boldsymbol{\alpha} \sim \boldsymbol{\lambda}^{\mathbf{p}}$ \\
\hline ZnO:Ga,Li \#7 & 18.1 & $6.5 \times 10^{19}$ & 2.3 \\
\hline ZnO:Ga,Li \#8 & 16.3 & $5.9 \times 10^{19}$ & 2.1 \\
\hline ZnO:Ga,Li \#9 & 9.75 & $3.5 \times 10^{19}$ & 2.8 \\
\hline ZnO:Ga,Li \#10 & 18.4 & $6.6 \times 10^{19}$ & 2.9 \\
\hline ZnO:Ga (reference) & 5.56 & $2 \times 10^{19}$ (from Hall) & 3.0 \\
\hline
\end{tabular}

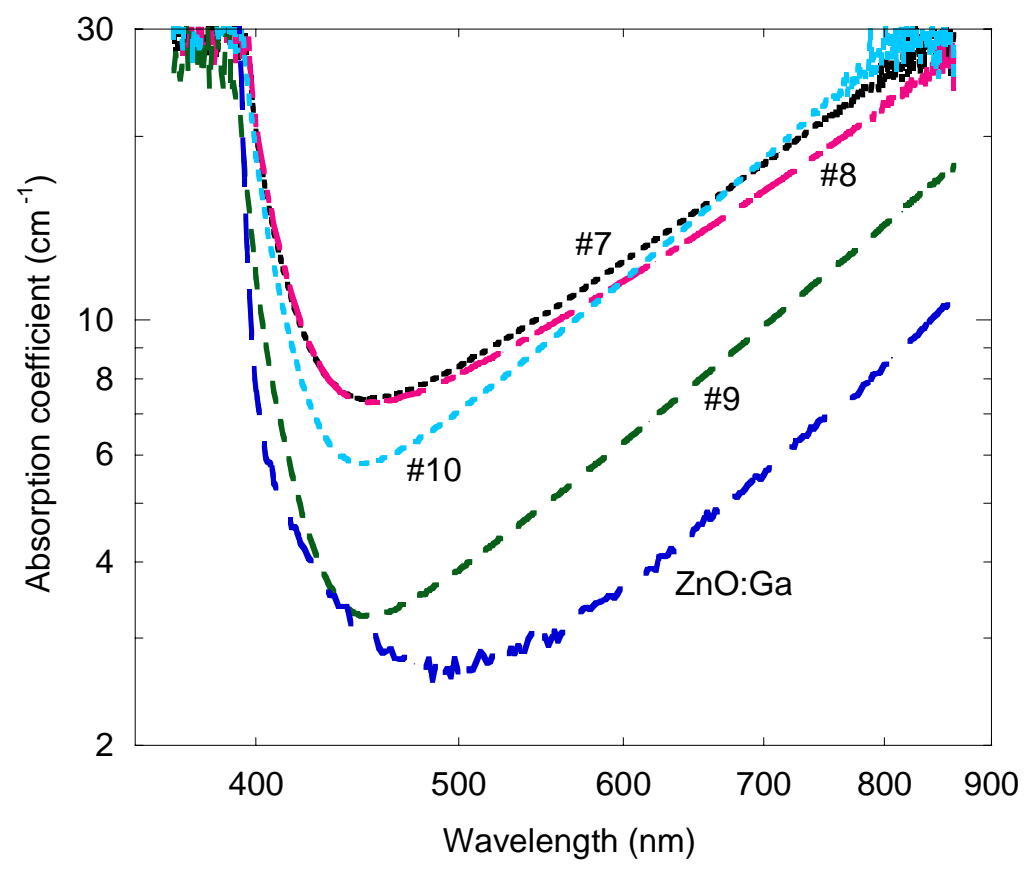

Figure 6.3 Absorption spectra of five Cermet samples shown in log-log scale. These data have been shown in Figure 6.2 in linear scale. Fitting results for $p$ value in $\alpha \sim \lambda^{\mathrm{p}}$ are presented in Table 6.1

Two other Cermet samples, one doped with Gd ( $\mathrm{ZnO}: \mathrm{Gd}$, Cermet \#3) and the other co-doped with Mg and Ga, (ZnO:Mg,Ga, Cermet \#2), have very light blue colors. The RT Hall carrier concentrations were $\mathrm{n}=2.3 \times 10^{18} \mathrm{~cm}^{-3}$ for the $\mathrm{ZnO}$ :Gd sample and $\mathrm{n}=3.5 \mathrm{x}$ $10^{18} \mathrm{~cm}^{-3}$ for the $\mathrm{ZnO}: \mathrm{Mg}, \mathrm{Ga}$ sample. FTIR spectra (Figure 6.4) show the free carrier absorption from about 0.9 to 2 microns, with the short-wavelength tail penetrating into the 
visible region, which explains the light blue colors. The power law fitting gave $p=3.6$ for these two samples.

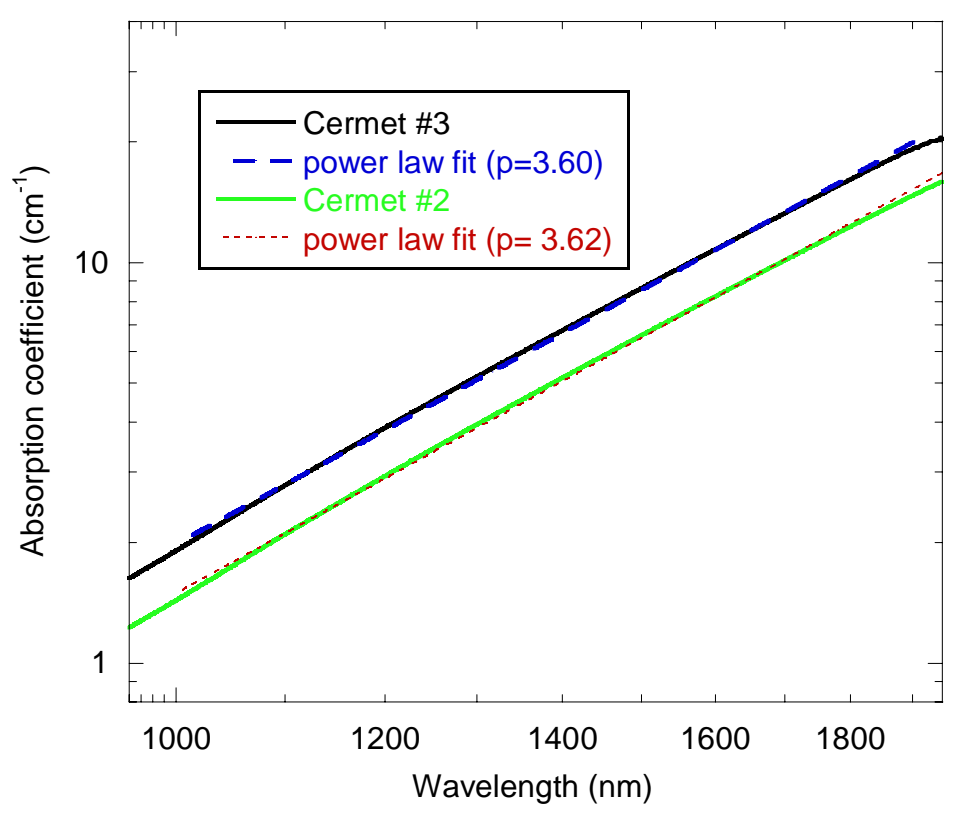

Figure 6.4 FTIR free carrier absorption from two single crystal $\mathrm{ZnO}$ samples Cermet $\# 2$ and Cermet \#3, and the power-law fittings. Notice the $\log -\log$ scale.

\subsection{Free Carrier Concentration Determined Using Infrared Reflectance Spectra}

Due to free carrier effects, the frequency dependence of the dielectric constant in a doped semiconductor can be written as

$$
\varepsilon_{r}(\omega)=\varepsilon_{o p t}\left(1-\frac{\omega_{p}^{2}}{\omega^{2}+i \omega / \tau}\right)
$$

where $\varepsilon_{\mathrm{opt}}=\mathrm{n}^{2}$ and $\omega_{\mathrm{p}}$ is the plasma frequency,

$$
\omega_{p}^{2}=\frac{N e^{2}}{\varepsilon_{o p t} \varepsilon_{0} m_{e}^{*}}
$$

In a lightly damped system, Eq. 6.2 can be simplified as $\varepsilon_{\mathrm{r}}(\omega)=\varepsilon_{\mathrm{opt}}\left(1-\omega_{\mathrm{p}}{ }^{2} / \omega^{2}\right)$. Because the reflectivity R depends on frequency as

$$
R=\frac{\left|\varepsilon_{r}^{1 / 2}-1\right|^{2}}{\left|\varepsilon_{r}^{1 / 2}+1\right|^{2}},
$$


the reflectance $\mathrm{R}=0$ if $\varepsilon_{\mathrm{r}}(\omega)=1$. This gives a frequency $\omega_{\text {opt }}$ just above the plasma frequency at which the reflectance spectra will have a minimum. In practice, $\mathrm{R}$ reaches a finite non-zero minimum. The frequency at which this occurs when a sample is surrounded by air is given by

$$
\omega_{o p t}^{2}=\frac{\varepsilon_{o p t}}{\varepsilon_{o p t}-1} \omega_{p}^{2}
$$

The relation given in Eq. 6.5 provides a way to determine the free carrier concentration from infrared reflectance spectra. Using $\lambda_{\mathrm{p}}=2 \pi \mathrm{c} / \omega_{\mathrm{p}}$ and $\lambda_{\mathrm{opt}}=2 \pi \mathrm{c} / \omega_{\mathrm{opt}}$, Eqs. 6.3 and 6.5 can be rearranged for the free carrier concentration $\mathrm{N}$,

$$
N=\frac{(2 \pi c)^{2}}{e^{2}} \frac{\left(\varepsilon_{o p t}-1\right) \varepsilon_{0} m_{e}^{*}}{\lambda_{o p t}^{2}}
$$

Here, $\mathrm{m}_{\mathrm{e}}{ }^{*}=0.24$ and $\varepsilon_{\mathrm{opt}}=3.7$ were used for $\mathrm{ZnO}$, and $\mathrm{N}=7.24 \times 10^{20} / \lambda_{\mathrm{opt}}{ }^{2}\left(\mathrm{~cm}^{-3}\right)$ where $\lambda_{\mathrm{opt}}$ is in microns. Figure 6.5 shows the FTIR reflectance spectra from three doped Cermet $\mathrm{ZnO}$ single crystal samples. From the measured reflectance minimum, $\lambda_{\text {opt }}=5.70$ micron for the $\mathrm{ZnO}: \mathrm{Ga}$ sample. This gave the free carrier concentration as $\mathrm{N}=2.23 \times 10^{19} \mathrm{~cm}^{-3}$, which is very close to the Hall measurement of $2.0 \times 10^{19} \mathrm{~cm}^{-3}$ for this sample. The sample Cermet \#7 is representative of the four dark blue Ga-Li codoped samples discussed above and shown in Figs. 6.2 and 6.3. The reflectance minimum for this sample was found at a shorter wavelength $\lambda_{\text {opt }}=3.98$ microns and $\mathrm{N}=4.57 \times 10^{19} \mathrm{~cm}^{-3}$ was obtained, fairly close to that estimated from the absorption coefficient $\left(6.5 \times 10^{19} \mathrm{~cm}^{-3}\right)$. The sample Cermet \#1 in Figure 6.5 is Mg-Ga codoped, one of seven lighter blue Cermet samples, two of which were discussed above and shown in Figure 6.4. The reflectance minimum from this sample occurred at $\lambda_{\text {opt }}=10.5$ microns, which corresponds to $\mathrm{N}=6.57 \times 10^{18} \mathrm{~cm}^{-3}$. 


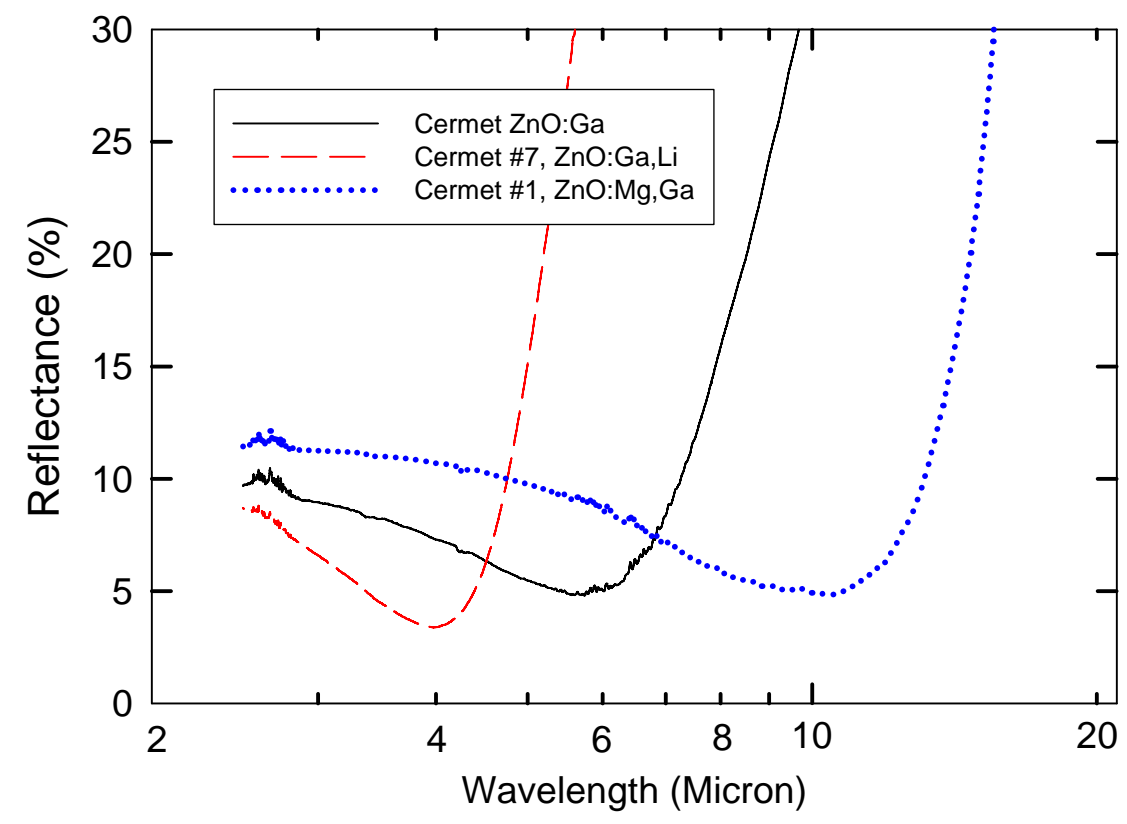

Figure 6.5 FTIR reflectance spectra from three n-type Cermet single crystal $\mathrm{ZnO}$ samples.

\subsection{Effects of Anneal Treatments on $\mathrm{ZnO}$}

Native defects may also contribute to the n-type conductivity in as-prepared $\mathrm{ZnO}$. Annealing of a $\mathrm{ZnO}$ single crystal sample grown from SCVT technique in $\mathrm{Zn}$ vapor has produced a higher n-type sample according to optical absorption and Hall measurements (Halliburton, 2005). In this section, I will discuss the effect of heat treatments on the optical absoption, Hall free carrier concetrantion, and PL emission.

A set of Cermet $\mathrm{ZnO}$ samples were prepared for a heat treatment study performed at Oak Ridge National Laboratory (ORNL) (Dr. Lynn Boatner, collaborator). Two 10 mm x 10 $\mathrm{mm}$ pieces of Cermet as-grown samples were cut into smaller pieces with dimensions of about $4.5 \mathrm{~mm} \times 4.5 \mathrm{~mm}$. One piece was kept untreated (referred to as CMT1), while the others were subjected to separate heating treatments. The samples were sealed in separate quartz ampoules under vacuum with $\mathrm{Zn}$ metal and heated to $1050{ }^{\circ} \mathrm{C}$ for 30 minutes. These samples all turned red after the heat treatment. One of these red samples was kept as a 
reference (CMT2), while the rest were subject to second-step treatments after being removed from the quartz ampoules:

Sample Second-Step Treatment Description

CMT3 Heated to $800{ }^{\circ} \mathrm{C}$ for one hour in flowing $\mathrm{H}_{2} / \mathrm{Ar}$; then cooled to RT with gas

flowing. The sample stayed red.

CMT5 Heated to $1000{ }^{\circ} \mathrm{C}$ for 2 hours in $\mathrm{H}_{2} \mathrm{O}$ vapor. The sample turned clear.

CMT7 Heated to $1000{ }^{\circ} \mathrm{C}$ for 2 hours with $\mathrm{O}_{2}$ gas flowing. The sample turned clear.

CMT8 Heated to $1000{ }^{\circ} \mathrm{C}$ for 2 hours in $\mathrm{D}_{2} \mathrm{O}$ vapor. The sample turned clear.

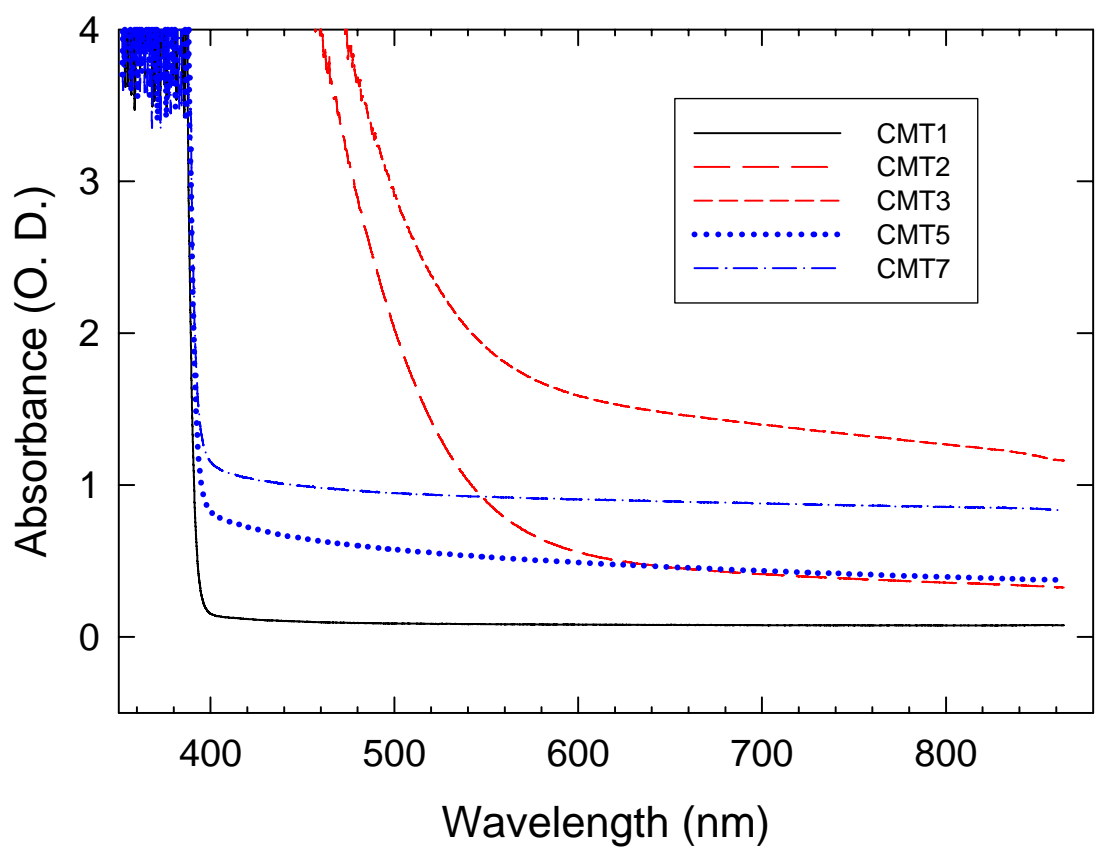

\section{Figure 6.6}

Absorption spectra of five Cermet singlecrystal $\mathrm{ZnO}$ samples. Heat treatments are discussed in the text.

Figure 6.6 shows representative UV-Vis absorption spectra from five of these samples. Since sample thickness is similar, the data is shown as measured in absorbance, rather than converted to absorption coefficient. The as-grown sample (CMT1) has an absorption edge at $3.18 \mathrm{eV}(390 \mathrm{~nm})$ and is highly transparent over the entire visible region. Samples CMT2 and CMT3 have absorption edges at about $460 \mathrm{~nm}$ and $470 \mathrm{~nm}$, respectively. 
This shift to longer wavelengths covering the blue spectral region is the cause of the red color of these two samples. After the second step of treatment, both CMT5 and CMT7 are examples of samples that turned back to clear. As seen in Figure 6.6, their absorption edges moved back to about $390 \mathrm{~nm}$.

The change of sample color was accompanied by an increase in the free carrier concentration. Room-temperature van der Pauw Hall measurements gave $\mathrm{n}_{\mathrm{H}}=7.7 \times 10^{16}$ $\mathrm{cm}^{-3}$ for the as-prepared sample CMT1. For the sample after the first-step (Zn vapor) treatment, $\mathrm{n}_{\mathrm{H}}=8.2 \times 10^{17} \mathrm{~cm}^{-3}$ was found for CMT2. The second-step treatment in sample CMT3 reduced the free carriers to $n_{H}=2.8 \times 10^{17} \mathrm{~cm}^{-3}$, still more than three times larger than that of the untreated sample CMT1. For the samples that turned clear, i.e., CMT5, CMT7, and CMT8, the second-step treatments brought $\mathrm{n}_{\mathrm{H}}$ down to mid- $10^{16} \mathrm{~cm}^{-3}$, close or lower than that for the untreated sample. Free carrier absorption spectra are consistent with the Hall measurements (not shown). The as-grown sample CMT1 has medium free carrier absorption for $\lambda>4 \mu$. The two red samples have strong free carrier absorption for $\lambda>2 \mu$. For the samples that turned clear after the second-step anneal treatment, CMT5 has medium absorption similar to CMT1, while the other two has no significant absorption till $\lambda>8 \mu$. Table 6.2 is a summary of the Hall measurement results and the color changes for these samples, along with a nominal description of their free carrier absorption.

RT PL emission spectra of the treated samples (not shown) changed significantly from the as-grown sample CMT1, but none is brighter than the latter. UV emission from the two red samples were several times weaker than that from CMT1, and a strong broad visible band appears at $510 \mathrm{~nm}$. For the samples that turned clear after second anneal, CMT5 is 
almost as bright as CMT1 in the UV, with a strong visible emission, the UV emission is almost eliminated from sample CMT7 and CMT8.

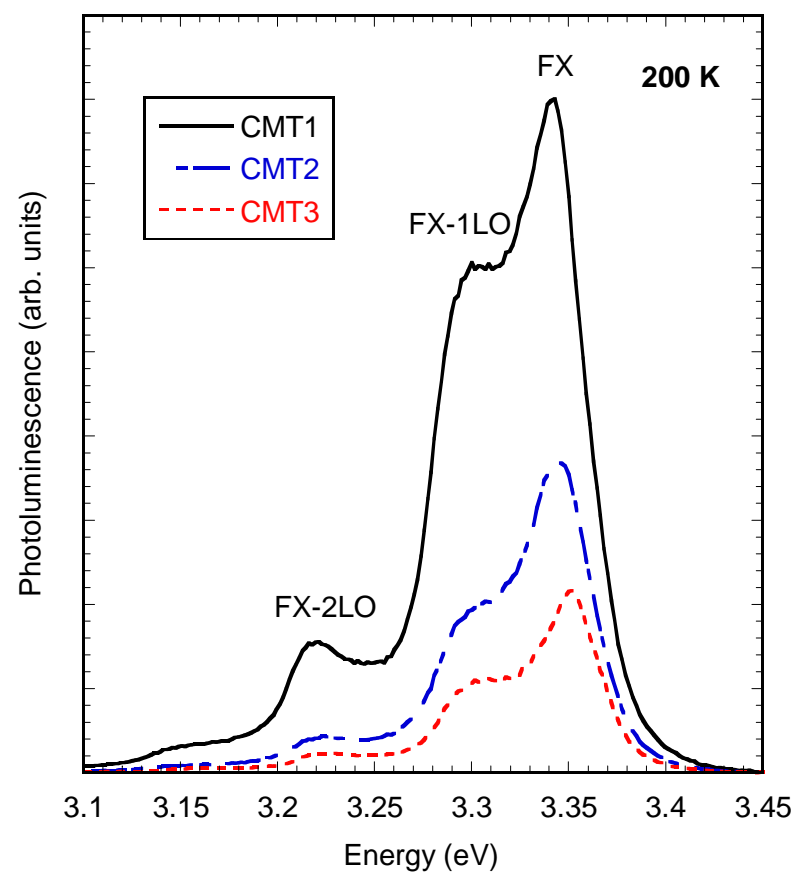

Figure 6.7 200-K PL from three HPMgrown samples.

Table 6.2 Free carrier concentration from Hall measurements, comparison of free carrier absorption, and color changes for 6 CMT samples.

\begin{tabular}{|c|c|c|c|}
\hline Sample & $\left.\mathbf{n}_{\mathbf{H}} \mathbf{( c m}^{-\mathbf{3}}\right)$ & $\begin{array}{c}\text { Free carrier } \\
\text { absorption }\end{array}$ & Color \\
\hline CMT1 & $7.7 \times 10^{16}$ & medium & red \\
\hline CMT2 & $8.2 \times 10^{17}$ & strong & red \\
\hline CMT3 & $2.8 \times 10^{17}$ & strong & clear \\
\hline CMT5 & $5.8 \times 10^{16}$ & medium & clear \\
\hline CMT7 & $3.9 \times 10^{16}$ & weak & clear \\
\hline CMT8 & $3.1 \times 10^{16}$ & weak & \\
\hline
\end{tabular}

The drastic reduction of UV emission from the two higher n-type (red) samples can be explained by the self-absorption effect. As seen from Fig. 6.6, the absorption edge for 
these samples red-shifted to $460-470 \mathrm{~nm}(2.7-2.6 \mathrm{eV})$. The RT PL from these samples, were due to FX and the phonon assisted FX transitions, as approved by the $200 \mathrm{~K}$ spectra shown in Fig. 6.7, suffered severe self-absorption, even though the data were taken using the reflection geometry. The cause of this red-shift of absorption edge that gives the samples the red color will be discussed next.

The red coloration was observed from a set of hydrothermally (HYD) grown $\mathrm{ZnO}$ samples (Airtron, NJ) after anneal treatments in a separate study. These samples were also provided from ORNL (Dr. Lynn Boatner). This sample set provides starting material that is much less n-type than crystals growing using the melt-growth technique. The HYD samples contain a variety of impurities but are closely compensated. As shown in Fig. 6.8, the asgrown sample $\left(\mathrm{n}_{\mathrm{H}}=2 \times 10^{13} \mathrm{~cm}^{-3}\right)$ has an absorption edge at $390 \mathrm{~nm}$ and is highly transparent in the visible region, similar to the untreated Cermet sample (CMT1). After the samples were heated in $\mathrm{Zn}, \mathrm{P}$, and $\mathrm{Mg}$ vapors, they turned red and the absorption edge shifted to above $500 \mathrm{~nm}$. From Hall measurements, the free carrier concentrations in these three samples were very similar with $\mathrm{n}_{\mathrm{H}}=(2.0-2.3) \times 10^{18} \mathrm{~cm}^{-3}$ measured at RT. The sample heated in vacuum for four hours stayed relatively clear, but an increase in free carriers to $\mathrm{n}_{\mathrm{H}}=2.3 \times 10^{17} \mathrm{~cm}^{-3}$ was measured. Compared to the CMT samples discussed earlier in this section, the set of Airtron samples gives a wider range of free carrier concentration from Hall measurement. The samples were polished on both sides at WVU and the absorption data were more reliable. Because the samples had quite different thicknesses, a conversion of the absorbance (in optical density, or OD) data to absorption coefficient (in $\mathrm{cm}^{-1}$ ) was performed in order to allow comparison between samples. 


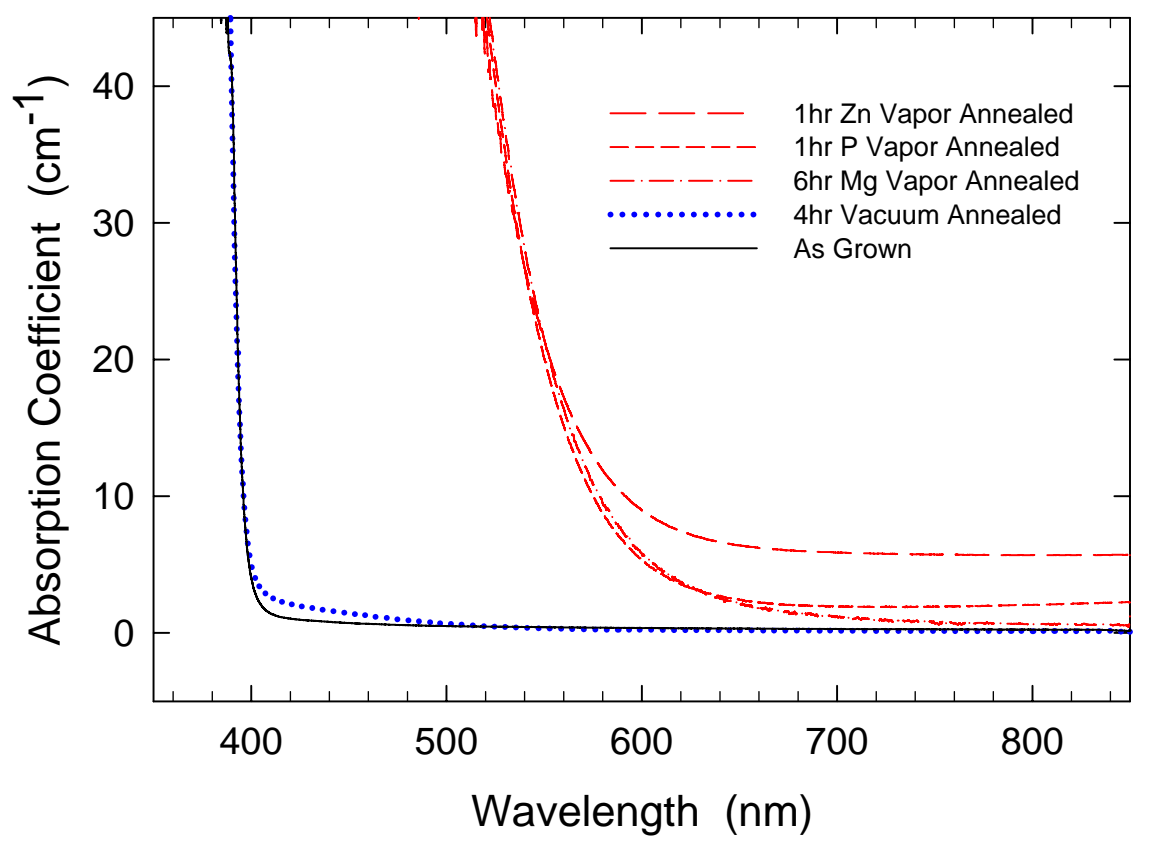

Figure 6.8

Absorption spectra of five hydrothermally grown Airtron single crystal $\mathrm{ZnO}$ samples provided by ORNL.

When the absorption data are presented in semi-logarithmic scales, as shown in Fig. 6.9, the absorption edge from the red samples have parabolic shapes and can be fit with a Gaussian line-shape, which has a peak value at $3.03 \mathrm{eV}(409 \mathrm{~nm})$ and a full width at half maximum of $0.78 \mathrm{eV}$. This is about $0.3 \mathrm{eV}$ below the bandgap. A study of high-temperature anneal in $\mathrm{Zn}$ vapor was performed on SCVT ZnO a few years ago (Halliburton, 2005), and this absorption band was observed along with significant increase in free carrier absorption after anneal. The authors proposed that oxygen vacancies $\left(\mathrm{V}_{\mathrm{O}}\right)$ produced during the anneal treatment in the reducing atmosphere were responsible for this below-bandgap absorption. Production of $\mathrm{V}_{\mathrm{O}}$ and related coloration were also reported in a more recent study on electron-irradiated $\mathrm{ZnO}$ (Kappers, 2008). In Fig. 6.9, it was noticed that the $3.03 \mathrm{eV}$ band also exists in the as-grown and the vacuum-annealed samples. This is consistent with the attribution of this absorption band to the native $\operatorname{defect}\left(\mathrm{V}_{\mathrm{O}}\right)$, as the reducing nature of the heat treatments increased the concentration of $\mathrm{V}_{\mathrm{O}}$ in the treated samples. 
From the Gaussian fitting, the peak intensities were obtained, and Smakula's equation (Markham, 1966) was used to calculate the concentration of the defect centers related to the discrete absorption band peaking at $3.03 \mathrm{eV}$ :

$$
\alpha_{m}=7.75 \times 10^{-18} \frac{1}{H} \frac{\left(2+n^{2}\right)^{2}}{n} f N_{0} .
$$

Here $\alpha_{\mathrm{m}}$ is the peak absorption coefficient from the Gaussian fit, $\mathrm{H}$ is the FWHM in $\mathrm{eV}, \mathrm{n}$ is the refractive index, $\mathrm{f}$ is the unitless oscillator strength, and $\mathrm{N}_{0}$ is the defect density contributing to the absorption band. Using $\mathrm{n}=2.4$ and $\mathrm{f}=0.8$ for $\mathrm{ZnO}$, Eq. 6.7 was rewritten as $\mathrm{N}_{0}=3.29 \times 10^{15} \alpha_{\mathrm{m}}\left(\mathrm{cm}^{-3}\right)$, with $\alpha_{\mathrm{m}}$ in $\mathrm{cm}^{-1}$. The $\alpha_{\mathrm{m}}$ values from the curve fitting and the subsequent calculated $\mathrm{N}_{0}$ using Eq. 6.7 for the five samples shown in Fig. 6.9 are listed in Table 6.3. The free carrier concentrations from Hall measurements $\left(\mathrm{n}_{\mathrm{H}}\right)$ are also listed.

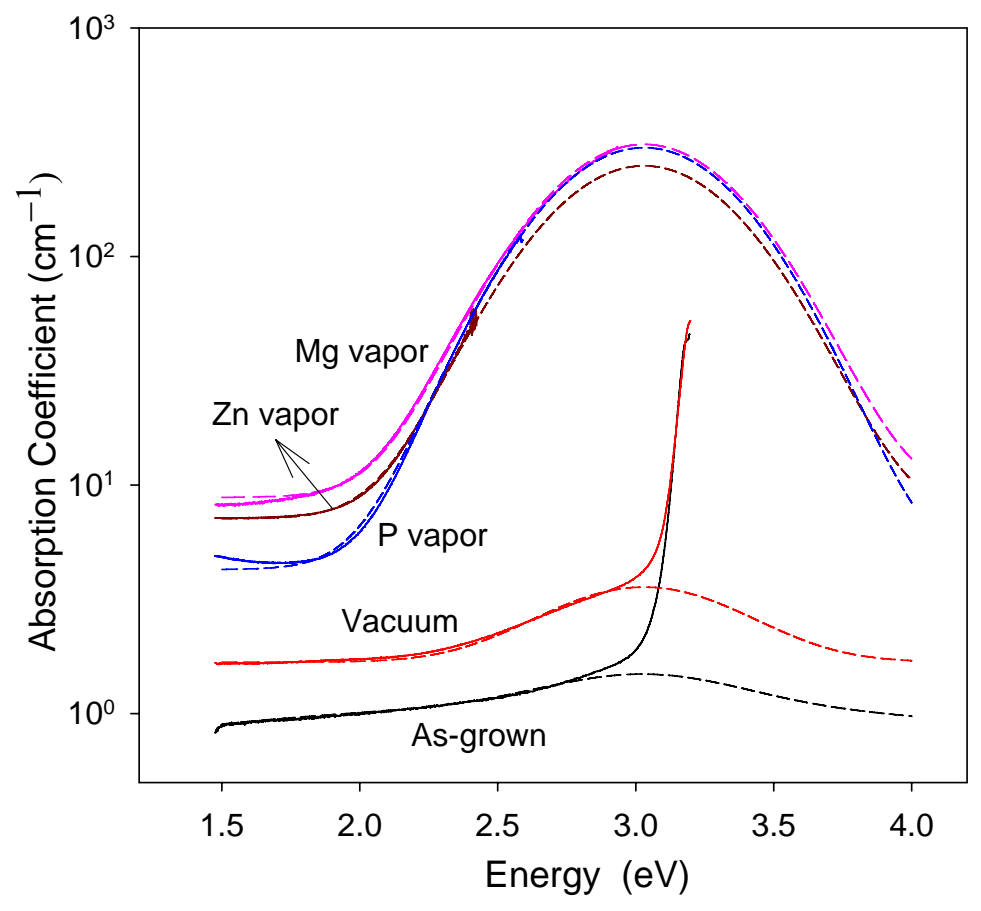

Figure 6.9 Gaussian lineshape fit to the absorption band centered at $3.03 \mathrm{eV}$ with a FWHM of $0.78 \mathrm{eV}$ from five hydrothermal $\mathrm{ZnO}$ samples (Airtron, NJ). Solid lines are the measured data, and the curve fits are shown as dashed lines.

The increase of free carriers after the anneal treatment cannot be explained by the induced $\mathrm{V}_{\mathrm{O}}$. As shown in Table 6.3, the Hall free carrier concentration $\left(\mathrm{n}_{\mathrm{H}}\right)$ for the three 
high n-type samples are about 2-3 times larger than the $\mathrm{V}_{\mathrm{O}}$ densities $\left(\mathrm{N}_{0}\right)$ in each sample.

For the vacuum annealed sample, $\mathrm{n}_{\mathrm{H}}$ is 40 times larger than $\mathrm{N}_{0}$. In the semi-insulate asgrown sample, $\mathrm{N}_{0}$ is nearly two orders larger than $\mathrm{n}_{\mathrm{H}}$, but such a small amount of $\mathrm{V}_{\mathrm{O}}$ can be more compensated by the acceptors existing in this HYD sample. Another reason that the $V_{O}$ may not be responsible for the free carriers is that its energy level is too deep in the bandgap, about $2.1 \mathrm{eV}$ below the CB (Evans, 2008). For the hydrothermal samples, the growth technique itself brings in high density of extrinsic donors and acceptors, but is closely compensated in many cases, as has been shown in Chapter 5. The production of native donors during the reducing anneal treatment provides additional compensation centers for acceptors, allowing some of the shallow donor levels to serve as active donor centers that contribute to the free carriers.

Table 6.3. Peak absorption coefficient $\left(\alpha_{\mathrm{m}}\right)$, defect density $\left(\mathrm{N}_{0}\right)$ calculated using Smakula's equation (Eq. 6.7), and Hall free carrier concentration $\left(\mathrm{n}_{\mathrm{H}}\right)$ for five hydrothermal Airtron $\mathrm{ZnO}$ samples.

\begin{tabular}{|c|c|c|c|}
\hline Sample & $\boldsymbol{\alpha}_{\mathbf{m}}\left(\mathbf{c m}^{-\mathbf{1}}\right)$ & $\mathbf{N}_{\mathbf{0}}\left(\mathbf{c m}^{-\mathbf{3}}\right)$ & $\mathbf{n}_{\mathbf{H}}\left(\mathbf{c m}^{\mathbf{3}}\right)$ \\
\hline As grown & 0.43 & $1.4 \times 10^{15}$ & $2.0 \times 10^{13}$ \\
\hline 4-hour vacuum & 1.95 & $6.4 \times 10^{15}$ & $2.3 \times 10^{17}$ \\
\hline 1-hour Zn vapor & 249 & $8.2 \times 10^{17}$ & $2.1 \times 10^{18}$ \\
\hline 1-hour P vapor & 295 & $9.7 \times 10^{17}$ & $2.3 \times 10^{18}$ \\
\hline 6-hour Mg vapor & 301 & $9.9 \times 10^{17}$ & $2.0 \times 10^{18}$ \\
\hline
\end{tabular}

In summary, high n-type single crystal $\mathrm{ZnO}$ samples were prepared through intentional doping and after-growth treatments, with starting sample grown by HPM and HYD methods. Colorations accompanying extrinsic doping and anneal treatments were explained using UV-visible-IR absorption spectra. The blue color from doped Cermet $\mathrm{ZnO}: \mathrm{Ga}, \mathrm{ZnO}: \mathrm{Ga}, \mathrm{Li}, \mathrm{ZnO}: \mathrm{Mg}, \mathrm{Ga}$, and $\mathrm{ZnO}: \mathrm{Gd}$ samples comes from the free carrier 
absorption tail that extends into the red-orange region. Absorption and reflectance data were used to get free carrier concentrations from these samples as Hall measurements cannot be conducted due to large sample dimensions. Several co-doped samples with $\mathrm{n}$ in the range of high $10^{18} \mathrm{~cm}^{-3}$ have bright UV emissions, while those $\mathrm{ZnO}: \mathrm{Mg}$, Ga samples that have $\mathrm{n}$ in the mid- $10^{19} \mathrm{~cm}^{-3}$ are less bright compared to the Cermet $\mathrm{ZnO}: \mathrm{Ga}$ sample studied in Chapter 4 . The red color of Cermet and Airtron samples after anneal treatments arises from a $3.03 \mathrm{eV}$ absorption band that extends into the blue spectra region, which is related to oxygen vacancies produced during the reducing atmosphere treatments. Smakula's Equation was employed to obtain the concentration of oxygen vacancy. Explanation of the increase of free carrier concentration after the anneal needs invoking of defects other than $\mathrm{V}_{\mathrm{O}}$. The high $\mathrm{n}$ type samples prepared by the anneal treatments are not good for UV scintillator applications, because the broad near-edge absorption band peaking at $3.03 \mathrm{eV}$ causes drastic reduction to the UV emissions that mainly from FX and its phonon lines. 


\section{Chapter 7 Evaluation of Powder and Ceramic ZnO for Scintillator Applications}

As discussed in Chapter 1, gallium-doped $\mathrm{ZnO}$ is being used as the scintillation material in the associated alpha particle detector in the D-T neutron generator at ORNL. For this purpose, the best candidate $\mathrm{ZnO}$ :Ga should have bright UV emission. The undesired visible emission should be eliminated or made as weak as possible. Besides single crystal samples such as those discussed in Chapter 4 and 6, powder $\mathrm{ZnO}$ :Ga samples have demonstrated very bright UV emissions (Neal, 2007). Ceramics with high UV luminescence could greatly ease the fabrication and lower the cost of the scintillator applications. Comparison of $\mathrm{ZnO}$ :Ga powder samples prepared at ORNL and those obtained from commercial sources were conducted at ORNL and WVU, with Ga doping levels varying from 80 ppm up to 4 at $\%$. Characterizations performed at ORNL included alpha-particle excitation, X-ray diffraction (XRD), scanning/transmission electron microscopy (SEM/TEM), and inductively coupled plasma (ICP) measurments. At WVU, study included PL and X-ray excited luminescence on these samples. The effects of anneal treatments such as in $\mathrm{H}, \mathrm{O}_{2}$, $\mathrm{H}_{2} \mathrm{O}$ vapor, and air, were evaluated. To optimize the use of $\mathrm{ZnO}$ in the alpha-particle detector application, the ultimate desired form of the samples is as a ceramic. Ceramic $\mathrm{ZnO}$ samples were prepared in this study using hot uniaxial pressing (HUP) at ORNL, and spark plasma sintering (SPS) technique at UC-Davis. The PL and X-ray excited luminescence characterizations were performed at WVU to evaluate the effects of starting powder, sintering technique, sintering conditions (e. g., temperature and pressure), and after-sintering treatment, on the luminescence of ceramics. (The results of the PL characterization of ZnObased powder and ceramic scintillators for application as alpha-particle detectors have been saccepted for publication in 2008 in IEEE Transactions of Nuclear Science (Neal, 2008). ) 


\subsection{PL Characterization of ZnO:Ga Powder Samples Prepared at ORNL}

$\mathrm{ZnO}$ :Ga powder samples were prepared at ORNL via four growth methods:

combustion, urea precipitation, solution phase, and solid state synthesis. In the combustion synthesis method, a mixture of an aqueous solution of zinc nitrate $\left(\mathrm{Zn}\left(\mathrm{NO}_{3}\right)_{2}\right)$ and gallium nitrate $\left(\mathrm{Ga}\left(\mathrm{NO}_{3}\right)_{3} \cdot 6 \mathrm{H}_{2} \mathrm{O}\right)$ and glycine $\left(\mathrm{NH}_{2} \mathrm{CH}_{2} \mathrm{COOH}\right)$ is heated until it reaches the autoignition temperature. This process is able to produce large amount of samples in a relatively short time period. The product is a fine nanocrystalline powder of complex chemistry with precise compositional control. However, due to particle agglomeration, size and shape control are limited, and additional processing such as milling is needed. Samples prepared by B. Armstrong using the combustion method have Ga doping levels of 160 and 320 ppm, and are named as the JNA series.

In the urea precipitation synthesis, precursor oxides $\left(\mathrm{ZnO}\right.$ and $\left.\mathrm{Ga}_{2} \mathrm{O}_{3}\right)$ are dissolved in water and nitric acid and heated to speed the dissolution. Urea is added to initiate the precipitation and to control the particle size. As in the combustion method, agglomeration occurs during the precipitation. Samples prepared by J. Ramey using the urea precipitation method have Ga doping levels of 80, 160, 320, and 1,600 ppm, and are named as JNR series.

The solution-phase synthesis starts with a mixture methanol solution of zinc acetate dihydrate $\left(\mathrm{Zn}\left(\mathrm{C}_{2} \mathrm{H}_{3} \mathrm{O}_{2}\right)_{2} \cdot 2 \mathrm{H}_{2} \mathrm{O}\right)$, gallium oxide $\left(\mathrm{Ga}_{2} \mathrm{O}_{3}\right)$, and potassium hydroxide $(\mathrm{KOH})$. Dropwise addition of methanol solution of $\mathrm{KOH}$ at $60{ }^{\circ} \mathrm{C}$ triggers the reaction and subsequent precipitation of zinc hydroxide $\left(\mathrm{Zn}(\mathrm{OH})_{2}\right)$. The colloidal $\mathrm{Zn}(\mathrm{OH})_{2}$ product is then dehydrated, resulting in nanocrystalline $\mathrm{ZnO}$. In the preparation of $\mathrm{ZnO}$ : Ga powders by this method, the amount of added $\mathrm{Ga}_{2} \mathrm{O}_{3}$ was kept either stoichiometric or a slight excess in dilute $\mathrm{KOH}$. The advantage of this method is the control of particle size and shape by varying the 
precursor's concentration and reaction time. A disadvantage of this method is the limited quantity of products at a given time. Samples prepared by B. Kesanli using this method have Ga doping levels varying from 80 , to $5,500 \mathrm{ppm}$, and are named as the JNK series.

Table 7.1: Phase purity, size control, and crystal parameters from $\mathrm{ZnO}$ :Ga powders prepared by combustion, urea precipitation, and solution phase synthesis.

\begin{tabular}{|c|c|c|c|c|c|c|}
\hline \multicolumn{2}{|c|}{ Synthesis method } & Sample & $\begin{array}{c}\text { Secondary } \\
\text { phase }\end{array}$ & $\begin{array}{l}\text { Crystallite } \\
\text { size (nm) }\end{array}$ & a $(\AA)$ & c $(\AA)$ \\
\hline \multirow{2}{*}{\multicolumn{2}{|c|}{$\begin{array}{l}\text { Combustion } \\
\text { synthesis }\end{array}$}} & JNA-2 & No & 30.3 & 3.2497 & 5.2048 \\
\hline & & JNA-3 & No & 58 & 3.2497 & 5.20497 \\
\hline \multirow{4}{*}{\multicolumn{2}{|c|}{$\begin{array}{l}\text { Urea precipitation } \\
\text { synthesis }\end{array}$}} & JNR-1 & No & 32.0 & 3.2498 & 5.2053 \\
\hline & & JNR-2 & No & 30.7 & 3.24969 & 5.20454 \\
\hline & & JNR-3 & No & 39.0 & 3.24955 & 5.2050 \\
\hline & & JNR-4 & No & 67 & 3.2498 & 5.2049 \\
\hline \multirow{7}{*}{$\begin{array}{l}\text { Solution } \\
\text { phase } \\
\text { synthesis }\end{array}$} & \multirow{4}{*}{$\begin{array}{c}\text { Slightly } \\
\text { excess } \\
\mathrm{Ga}_{2} \mathrm{O}_{3} \text { in } \\
\mathrm{KOH}\end{array}$} & JNK-1 & No & 7.3 & 3.258 & 5.212 \\
\hline & & JNK-2 & No & 6.9 & 3.257 & 5.208 \\
\hline & & JNK-3 & No & 7.4 & 3.258 & 5.208 \\
\hline & & JNK-4 & Yes & 7.6 & 3.2551 & 5.2032 \\
\hline & \multirow{3}{*}{$\begin{array}{c}\text { Stoichio- } \\
\text { metric } \\
\mathrm{Ga}_{2} \mathrm{O}_{3} \text { in } \\
\mathrm{KOH}\end{array}$} & JNK-5 & No & 14.8 & 3.2511 & 5.2069 \\
\hline & & JNK-6 & No & 23.3 & 3.2506 & 5.2067 \\
\hline & & JNK-7 & No & 15.9 & 3.2517 & 5.2071 \\
\hline
\end{tabular}

Phase purity, sample size control, and crystalline parameters of samples prepared by the above three synthesis techniques are obtained through TEM (performed by Jane Howe) and XRD (performed by Cutler and Rawn) measurements at ORNL, and shown in Table 7.1. The only sample from which a second phase $\left(\mathrm{ZnGa}_{2} \mathrm{O}_{4}\right)$ is observed is the JNK-4 sample from solution synthesis, which has a Ga doping level of 5,500 ppm. The average particle sizes of the solution-synthesized samples are smaller than those from the other two synthesis methods. The JNK samples, especially those synthesized with excess $\mathrm{Ga}_{2} \mathrm{O}_{3}$ in dilute $\mathrm{KOH}$, have large crystal parameters a and c. 
The as-prepared powder samples were initially white in color. A portion of each sample was subjected to a hydrogen-anneal treatment at ORNL. The samples were heated up and held at $800{ }^{\circ} \mathrm{C}$ for $30 \mathrm{~min}$ before cooled down under a forming gas (4\% hydrogen and $96 \%$ argon) environment. After the H-anneal treatment, some of them changed to blue or gray colors while others remained white. TEM measurements show significant grain size increase after the anneal treatments. PL characterization shows improved UV emission and reduced visible emission, as desired for scintillation applications. Figure 7.1 shows the 300K PL spectra from samples JNA-2 and JNA-3 prepared by combustion synthesis, before (a) and after (b) hydrogen anneal treatment. The two samples were nominally doped with 160 ppm and 320 ppm of gallium, respectively. Inductively coupled plasma (ICP) measurement (performed by Banu Kesanli) found 698 ppm of Ga in JNA-2 and 1190 ppm of Ga in JNA-3. The as-prepared samples both feature bright visible emission peaking around $650 \mathrm{~nm}$, and a weak uv peak at about $380 \mathrm{~nm}$. After the H-anneal, the visible emissions dramatically decreased and the UV emission intensity increased by more than 10 times in both samples.

Figure 7.2 shows the $300-\mathrm{K}$ PL spectra from four JNR samples synthesized by the urea precipitation method. The as-prepared powders have almost no uv emission. The visible emissions are dominated by a green band peaking at about $520 \mathrm{~nm}$. After the hydrogen anneal, uv emission at about $387 \mathrm{~nm}$ become dominant. Reduction of the visible emission is not as drastic as in the JNA series. ICP measurement found $\mathrm{Cu}$ concentrations in these samples to be 912 ppm (JNR-1), 1060 ppm (JNR-2), 1554 ppm (JNR-3), and 2220 ppm (JNR-4), which are about 2-5 times larger than those found in the JNA samples. 


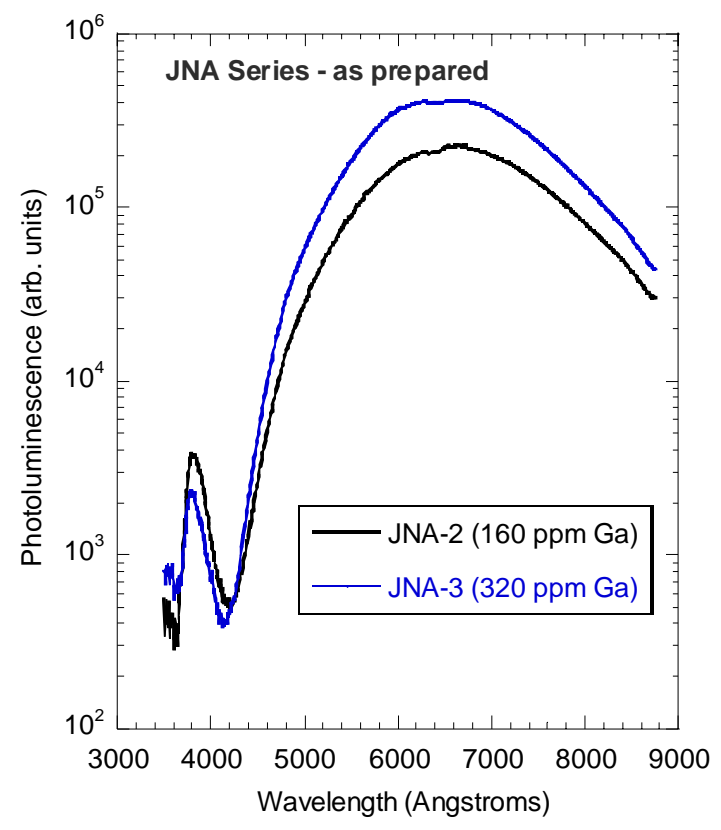

(a)

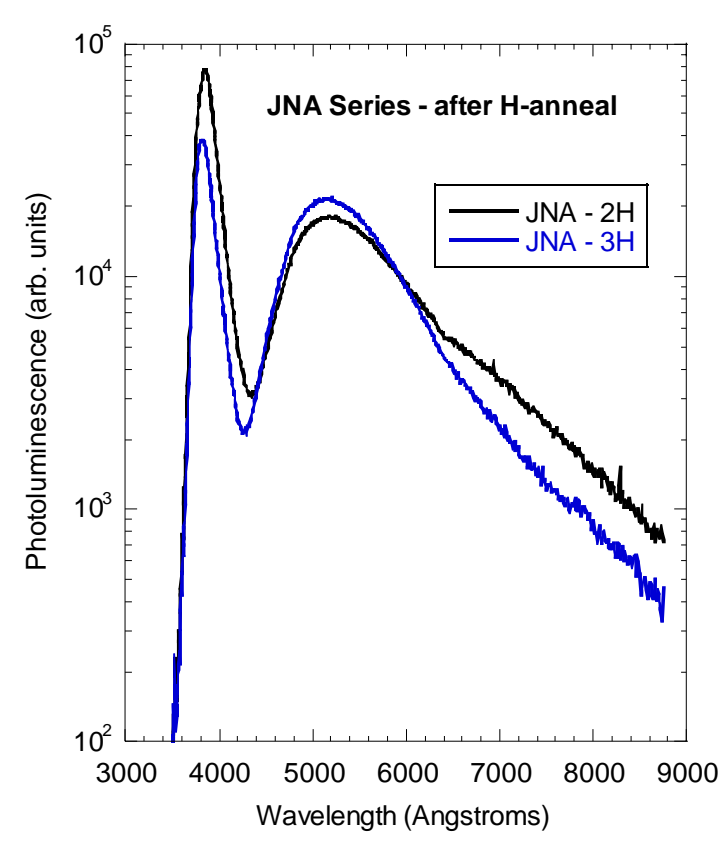

(b)

Figure 7.1 PL spectra at $300 \mathrm{~K}$ from combustion-synthesized samples JNA-2 and JNA-3 before (a) and after (b) hydrogen anneal treatment. Notice the logarithmic vertical scale.

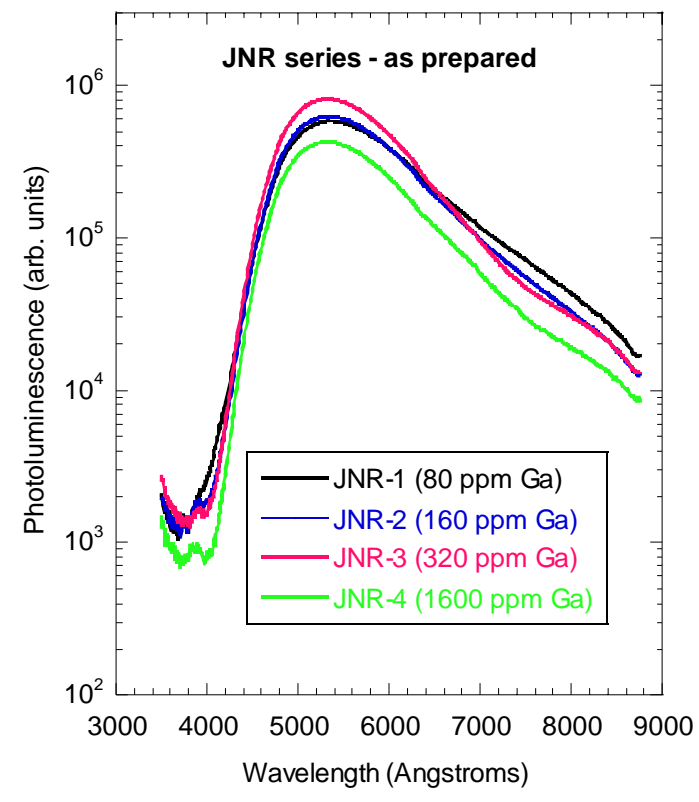

(a)

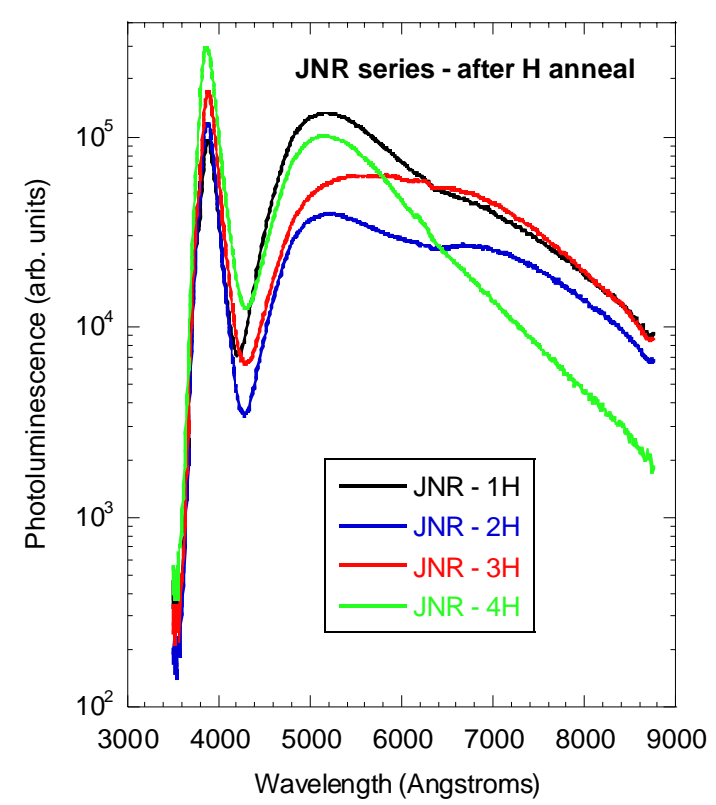

(b)

Figure 7.2 PL spectra at $300 \mathrm{~K}$ from JNR samples prepared by urea precipitation synthesis before (a) and after (b) hydrogen anneal treatment. Notice the logarithmic vertical scale. 
To understand the origin of the strong visible emission bands, PL spectra were taken at $5 \mathrm{~K}$ from the as-prepared JNR-2 sample. As shown in Figure 7.3, the 5-K emission (a, black solid line) has a peak at about $570 \mathrm{~nm}$. The short-wavelength side of the broad visible band has a multi-step feature, which can be fit with the structured green emission peaking at $510 \mathrm{~nm}$ (b, green solid line) associated with a phonon-assisted, localized excitation of substitutional $\mathrm{Cu}^{2+}$ ions in $\mathrm{ZnO}$, as first reported by Dingle (Dingle, 1969) and reaffirmed recently in present-day material (Garces, 2002). The main contributor to the visible PL peak is a yellow band peaking at about $600 \mathrm{~nm}$ (c, black dotted line), which has been assigned to a DAP transition involving a neutral shallow donor and a neutral Li acceptor (Giles, 2007). The third component on the longer wavelength side peaks at about $730 \mathrm{~nm}$ at $5 \mathrm{~K}(\mathrm{~d}$, red dashed line) and is most likely due to a DAP transition related to Zn vacancies (Kappers, 2008). The blue dashed line in Fig. 7.3 is the sum of these three components, and it reproduced the measured spectrum (a, black solid line) very well.

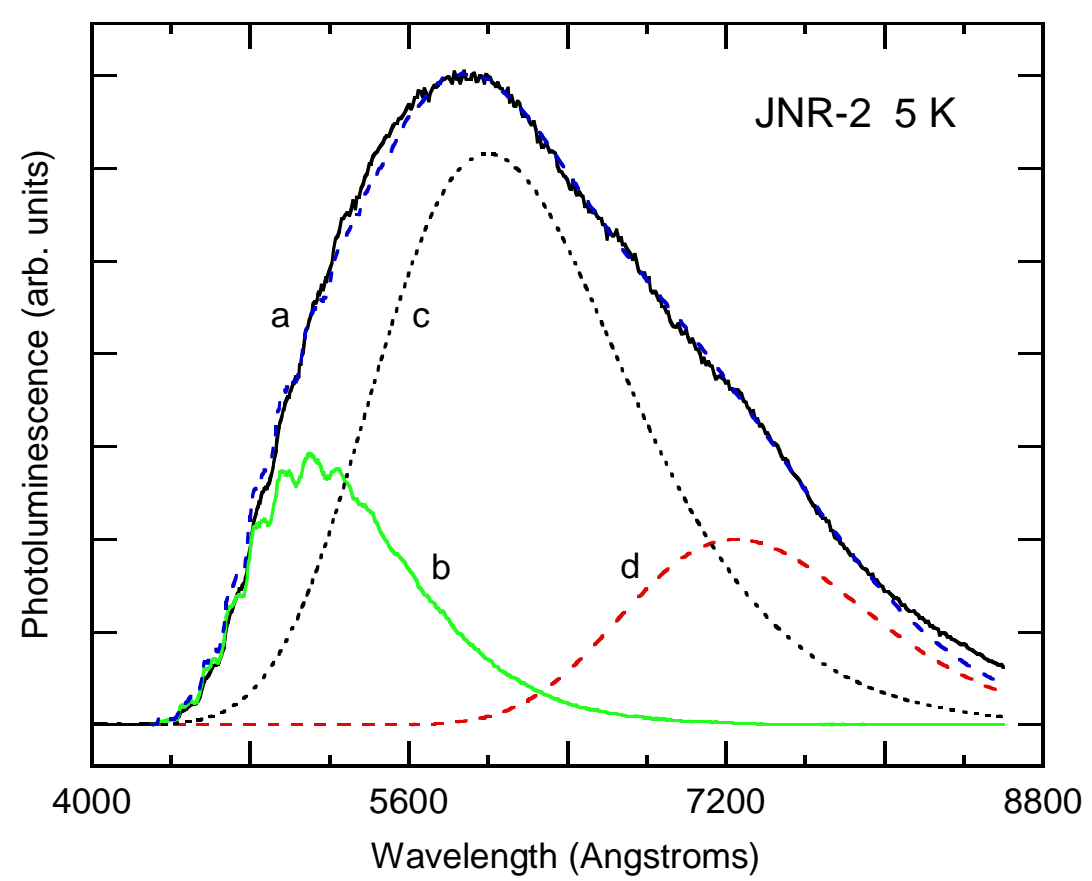

Figure 7.3 5-K PL spectra (a, black solid line) from sample JNR2 and a curve fit (dashed line) with three separate components:

(b) the structured emission related to $\mathrm{Cu}^{2+}$ ions (Garces, 2003), (c) DAP band related to neutral $\mathrm{Li}$ acceptor (Giles, 2007), and (d) DAP band related to $\mathrm{Zn}$ vacancy (Kappers, 2008). 
The 300-K emission from this sample (Fig. 7.2a, blue curve) has three components. The main peak is the superposition of the unstructured green DAP band related to a $\mathrm{Cu}$ acceptor (Garces, 2003), and the yellow DAP band related to Li acceptor (Giles, 2007). The third component is the red DAP band associated with the $\mathrm{Zn}$ vacancy (Kappers, 2008). The hydrogen anneal significantly reduced the yellow band (Fig. 7.2b, blue curve). This could occur if the hydrogen atmosphere was able to allow formation of $\mathrm{LiOH}^{-}$complexes which are electronically inactive, as shown from work on single crystals (Halliburton, 2004). As a consequence of the dramatic reduction of one of the major visible emission components, the UV edge emission, which had to compete with the slow DAP transitions, was enhanced. Enhancement of the UV emission would aid use of these powders in scintillator applications, thus, hydrogen anneal treatments appear to be effective in improving the UV scintillation when impurities such as Li are initially present.

Figure 7.4 shows the $300-\mathrm{K}$ PL from seven JNK powder samples prepared by the solution phase synthesis. The preparation conditions allow these samples to be divided into two groups. In one group (JNK-1 through JNK-4), samples were prepared with a slight excess of $\mathrm{Ga}_{2} \mathrm{O}_{3}$ in $\mathrm{KOH}$. The as-prepared samples show moderate visible emissions peaking around $600 \mathrm{~nm}$, while the UV emissions are about one order weaker (Fig. 7.4a). The second group includes three samples labeled JNK-5, -6, and -7 which were prepared with a stoichiometric amount of $\mathrm{Ga}_{2} \mathrm{O}_{3}$ in $\mathrm{KOH}$. The as-prepared samples in this group show stronger emissions both in the visible and in the UV region than those from the first group. ICP measurements found that the $\mathrm{Cu}$ content in these seven samples are lower than those in the JNA samples. The PL spectra are consistent with the ICP measurement, as the "copper green" band is not dominant in these samples. 


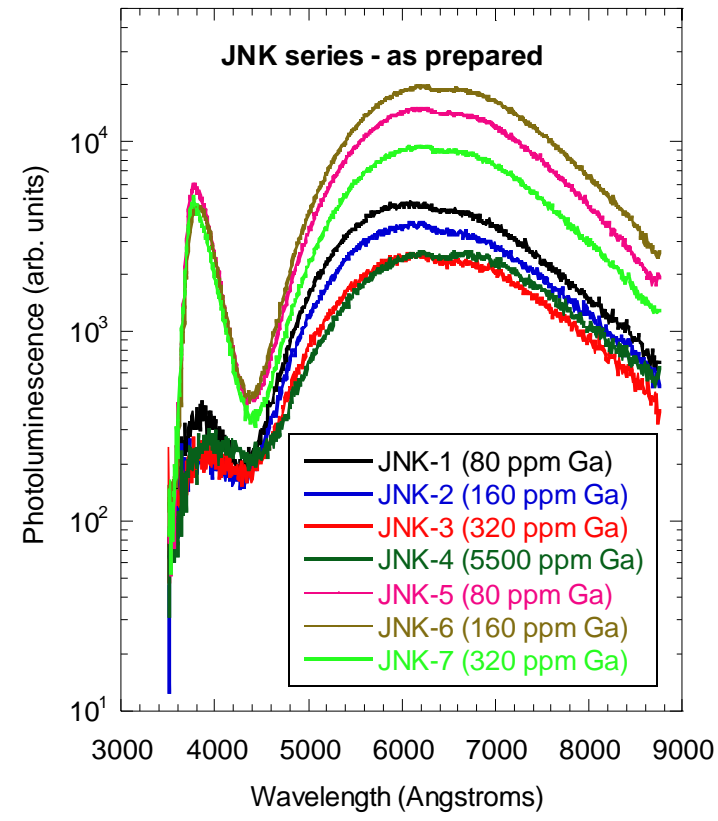

(a)

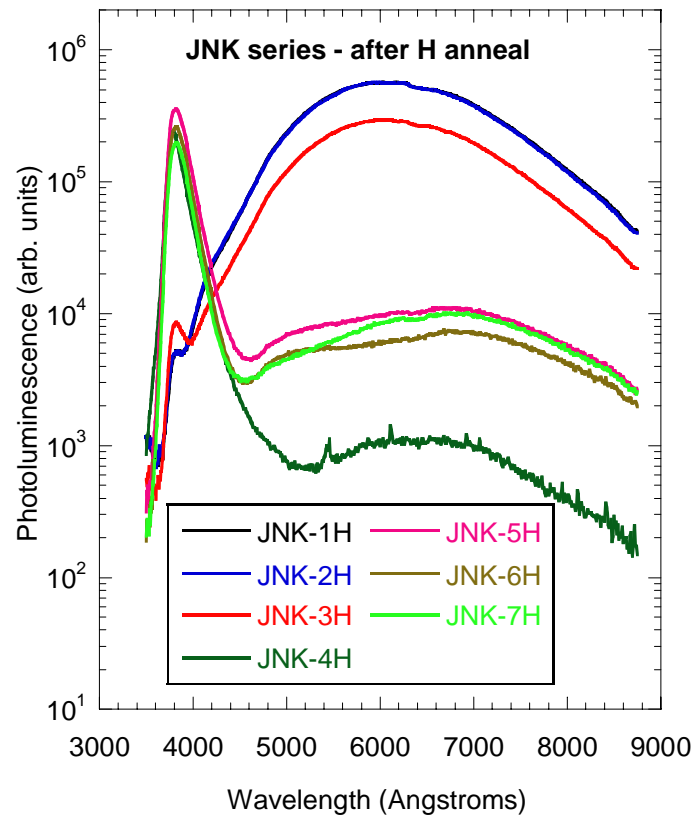

(b)

Figure 7.4 PL spectra at $300 \mathrm{~K}$ from JNK samples prepared by solution phase synthesis before (a) and after (b) hydrogen anneal treatments. Notice the logarithmic vertical scale. The spectra from sample JNK-6 and JNK-6H (brown curves) will be shown again in Fig. 7.5.

After H-anneal, the PL spectra are modified. The visible emissions from samples JNK-1H,-2H, and $-3 \mathrm{H}$ were enhanced by about 2 orders, while the UV emissions show no significant enhancement (Fig. 7.4b). On the other hand, the visible emissions from samples JNK-5, -6, and -7 were reduced, especially in the yellow band region, due to passivation of the Li acceptors by protons, as happened to the JNR samples. The green band related to $\mathrm{Cu}$ acceptor and the red band related to $\mathrm{Zn}$ vacancies did not change significantly, which may indicate that the hydrogen dose not sufficiently passivate or reduce such acceptors. As a consequence of the reduction of visible emissions, the UV emissions from samples JNK-5H, $6 \mathrm{H}$, and $7 \mathrm{H}$ were enhanced by about 2 orders. The UV-to-visible intensity ratios from these samples are now comparable to some single crystals such as as-grown Eagle-Picher samples. 
To compare with the H-anneal effect, air-anneal treatment was performed at WVU on five powder samples selected from the samples listed in Table 1. The samples were heated in an open-ended quartz tube extending through a small horizontal furnace and held in air at $800{ }^{\circ} \mathrm{C}$ for $30 \mathrm{~min}$ before quickly cooled to room temperature. The as-prepared samples were white, and all turned yellow after air-anneal. Figure 7.5 shows the effects of hydrogen and air anneal treatments on the 300-K PL spectra from sample JNK-6. The PL spectra from the as-prepared sample (Fig. 7.5, curve a) and after H-anneal (Fig. 7.5, curve b) have been shown in figure 7.4 (Fig. 7.4, brown curves). In contrast to the reduction of yellow band from H-anneal, the air-anneal treatment (Fig. 7.5, curve c) enhanced the visible emission, and the peak of the broad visible band moved to shorter wavelength $(570 \mathrm{~nm})$. A possible explanation is that anneal in air lowered the Fermi level and favored the existence of copper in the form of $\mathrm{Cu}^{2+}$ rather than $\mathrm{Cu}^{+}$, and the localized internal transition of $\mathrm{Cu}^{2+}$ ions that gives rise to the structured green band was enhanced, although the multi-phonon structure is not observable at room temperature.

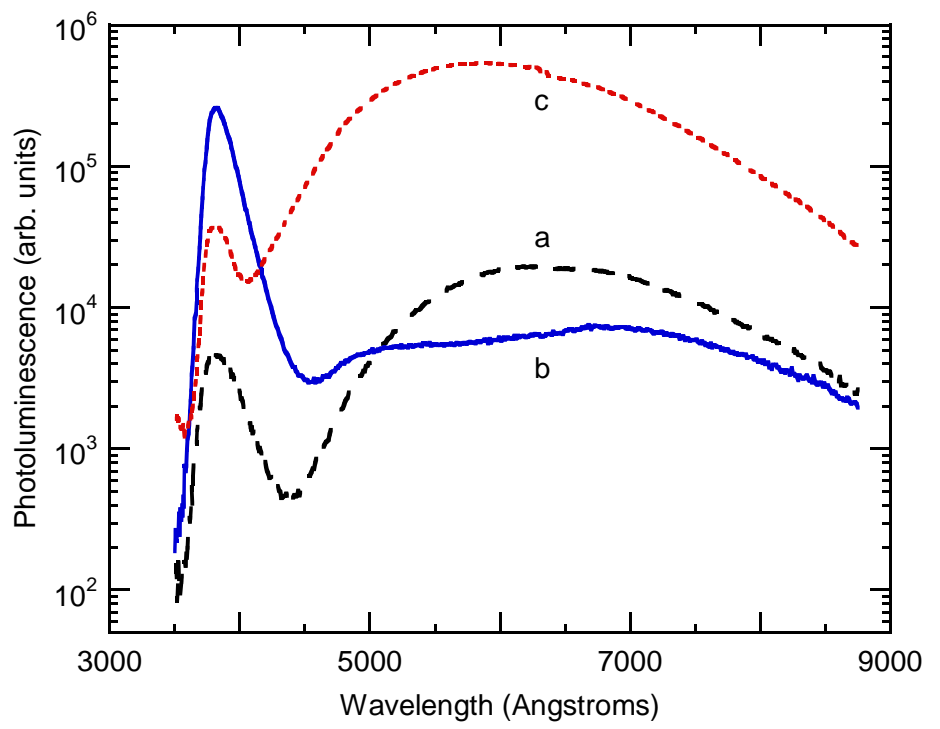

Figure 7.5. 300-K PL from ZnO:Ga powder sample JNK-6. (a) as-prepared, (b) after hydrogen anneal, and (c) after air anneal. Notice the logarithmic vertical scale. 
The most dramatic change after hydrogen anneal treatment occurred in sample JNK4H. This sample was was nominally doped with $5,500 \mathrm{ppm}$ of Ga. The ICP measurement found 7,350 ppm of $\mathrm{Ga}$ and $329 \mathrm{ppm}$ of $\mathrm{Cu}$ in the as-prepared sample (JNK-4). TEM measurements indicated formation of a second phase in this sample. The PL spectrum (Fig. 7.4a, dark green) is similar to those from samples JNK-1, -2 , and -3 . After the $\mathrm{H}$-anneal, the PL spectrum has a strong UV emission and a much reduced visible emission. The visible emission (Fig. 7.4b, dark green) is much weaker than those from samples JNK-5H, $-6 \mathrm{H}$, and $-7 \mathrm{H}$. The UV-to-visible intensity ratio is about 200 . This result encouraged the attempt of higher level of gallium doping. Using the same solution phase synthesis, sample JNK-8 was prepared with a Ga doping of 15,000 ppm. The as-prepared sample has a white color. After hydrogen anneal treatment, it turned into a light blue color. Figure 7.6 shows the TEM images taken at ORNL of the as-prepared sample (JNK-8) and after H-anneal (JNK-8H). The particle sizes before anneal are relatively uniform and have a size range of 7 to $15 \mathrm{~nm}$. After the $\mathrm{H}$ anneal, particle sizes increased significantly to a range of $20 \mathrm{~nm}$ to $80 \mathrm{~nm}$.

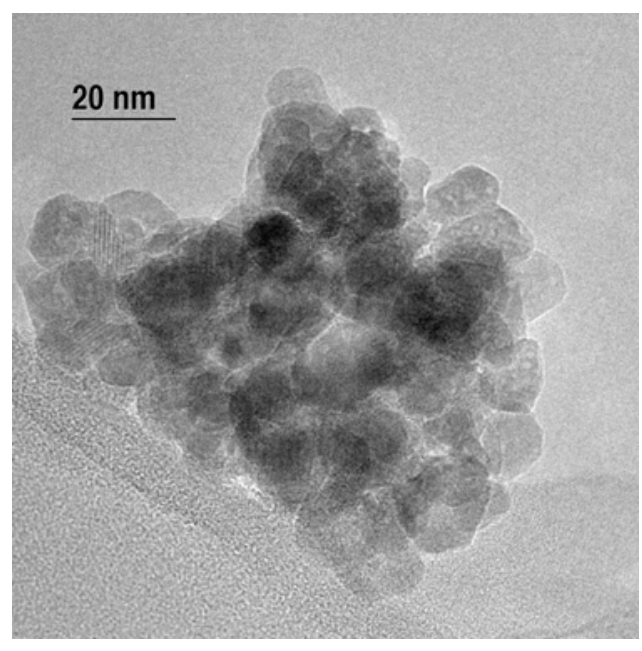

(a)

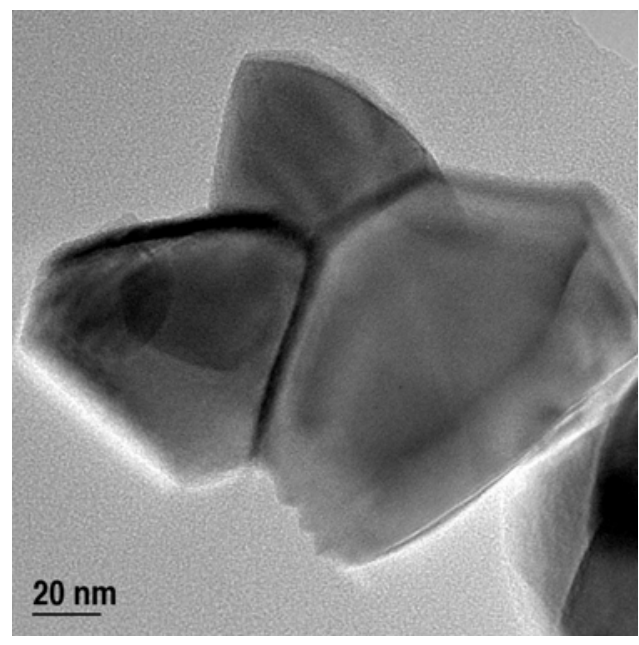

(b)

Figure 7.6 TEM images of as-prepared sample JNK-8 (a) and after H-anneal JNK-8H (b). Notice the particle size change. 
Figure 7.7(a) shows the 300-K PL spectra from the JNK-8 and JNK-8H samples. The as-prepared sample has strong visible emission and weaker UV emission, similar to those from JNK-5, -6, and -7. After H-anneal, the visible emission was reduced by about 20 times in intensity and the UV intensity increased by more than two orders. The UV-to-visible intensity ratio after $\mathrm{H}$-anneal is about 1000 , the highest of all the samples prepared using the three synthesis methods discussed above. The absolute intensity of UV emission from the JNK-8H sample, though, is still about 20 times dimmer than the reference W. Lehmann powder (labeled as WL1201 and not shown here, but will be discussed later in section 7.3).

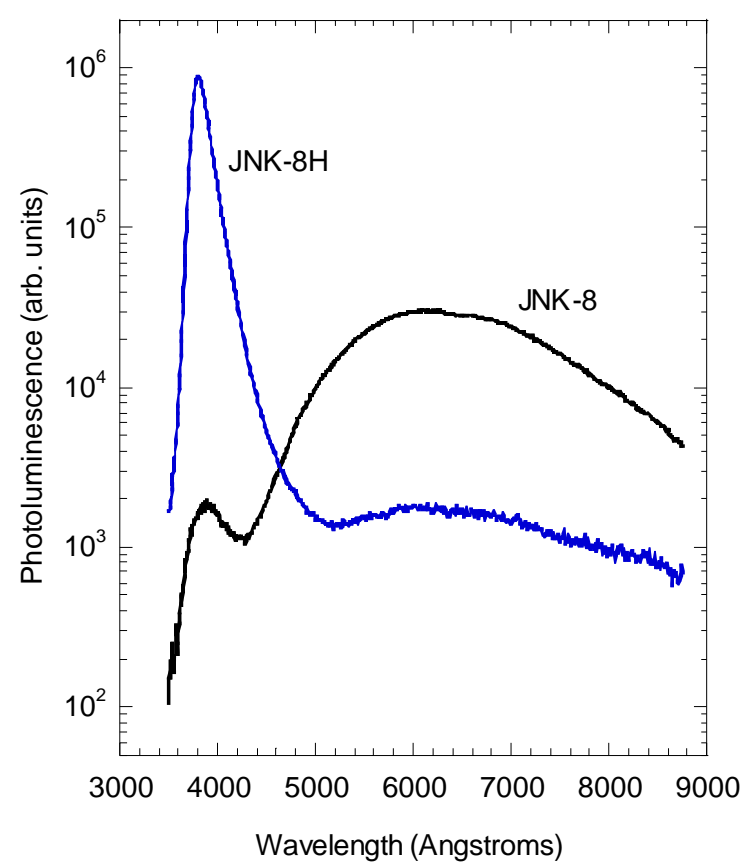

(a)

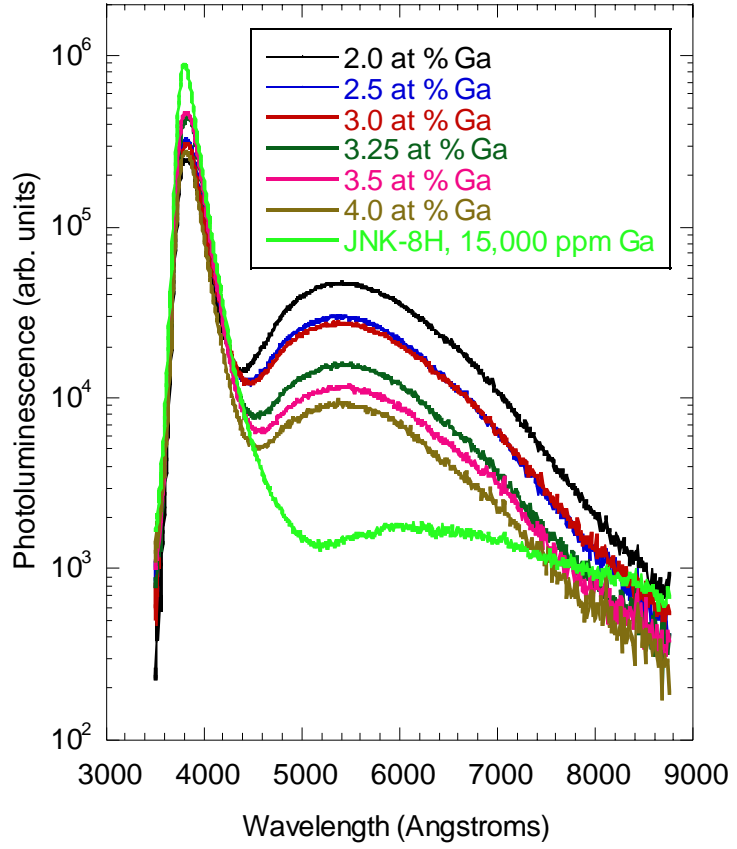

(b)

Figure 7.7 PL spectra at $300 \mathrm{~K}$ from $\mathrm{ZnO}$ :Ga samples with high levels of Ga-doping. (a) Solution-phase synthesized, doped with 15,000 Ga, as-prepared (JNK-8) and after H-anneal (JNK-8H); (b) solid-state synthesized, doped with 2-4 at \% Ga and after H-anneal, compared with JNK-8H. Notice the logarithmic vertical scale.

Higher Ga doping levels were conducted using solid-state synthesis by Cutler at ORNL. In this synthesis, commercial $\mathrm{ZnO}$ powder (Alfa Aesar) was mixed with $\mathrm{Ga}_{2} \mathrm{O}_{3}$ 
powders and heated. The product is powder ZnO:Ga. Fig. 7.7(b) shows the 300-K PL spectra from samples prepared using the solid-state synthesis having nominal Ga doping levels from 2.0 at $\%$ to 4.0 at $\%$. Spectra are shown for both before and after the $\mathrm{H}$-anneal treatments. The spectrum from JNK-8H is shown again for comparison. The as-prepared solid-state samples have PL spectra similar to those from samples JNK-5, -6, and -7 (Fig. 7.3(a)). The $\mathrm{H}$ anneal reduces the visible emission and enhanced the UV peak, but none of them have a UV-to-visible intensity ratio close to that of sample JNK-8H.

In summary for this section, combustion, urea-precipitation, solution-phase, and solid-state synthesis methods were used to prepare $\mathrm{ZnO}$ :Ga powder samples at ORNL, with Ga doping levels varying from $80 \mathrm{ppm}$ up to 4 at $\%$. PL spectra from the as-prepared samples show moderate or strong visible emissions but weak UV emissions. After being thermally annealed in hydrogen at $800{ }^{\circ} \mathrm{C}$ for $30 \mathrm{~min}$, these samples show substantial improvement in UV emissions and reduction in visible emissions. The solution-phase and solid-state syntheses with Ga doping levels of 5,500 ppm and higher and after combined with $\mathrm{H}$-anneal treatments produced $\mathrm{ZnO}:$ Ga powder samples with bright UV emission. Among these samples, JNK-8H (doped with 15,000 ppm Ga) has the largest uv-to-visible intensity ratio and shows promise for scintillator applications.

\subsection{PL Characterization of Commercial ZnO Powder Samples}

Besides samples synthesized at ORNL, commercial $\mathrm{ZnO}$ powder samples were also evaluated for the application as scintillators. In this work, powder $\mathrm{ZnO}$ samples of different purity levels were obtained from two sources, Alfa Aesar (AA) and Sigma Aldrich (SA). The as-prepared commercial samples show a large variation in PL spectra. Samples from the 
same vendor with different purity levels, or even with same purity level but bearing different lot numbers (AA), or batch numbers (SA) have very different emission spectra.

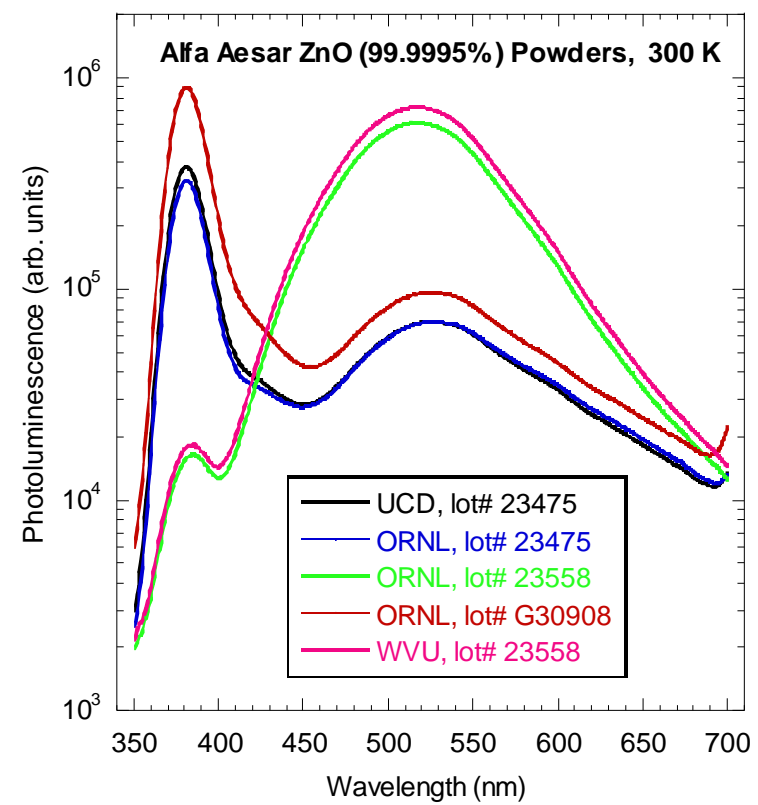

(a)

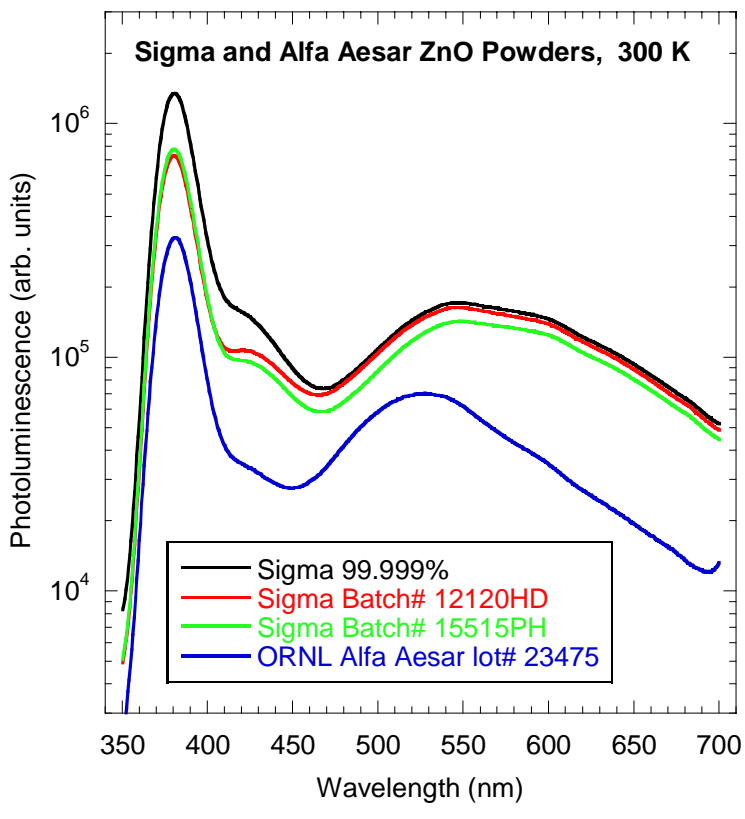

(b)

Figure 7.8 (a) PL spectra at $300 \mathrm{~K}$ from Alfa Aesar (AA) ZnO powder samples $(99.9995 \%$ pure) with different lot numbers. (b) PL spectra at $300 \mathrm{~K}$ from Sigma Aldrich (SA) ZnO powder samples (99.999\% pure) with different batch numbers, in comparison with one of the AA samples shown in (a). Notice the logarithmic vertical scales.

Figure 7.8(a) shows the 300-K PL spectra from five AA powder samples purchased at different times by ORNL, UC-Davis, and WVU. These samples have the same purity classification (99.9995\%) but have different lot numbers. Some of them show bright UV emissions, while the others have broad, strong visible emissions with the green band being dominant. Copper contamination may be responsible for this undesired emission. Although the chemical analysis report accompanying the sample provided no measurement on $\mathrm{Cu}$ content, the ICP analysis performed at ORNL found $634 \mathrm{ppm}$ of $\mathrm{Cu}$ from one of the AA powder samples. Figure 7.8(b) shows the 300-K PL spectra from three Sigma Aldrich (SA) $\mathrm{ZnO}$ powder samples and one of the AA samples is included for comparison. All three SA 
samples show bright UV emissions, about one order brighter than the visible emissions, which is more promising than the AA samples. The multi-component visible band has a main peak around $550 \mathrm{~nm}$, and a side peak about $600 \mathrm{~nm}$ (yellow band). A separate peak shows up in the 400-450 $\mathrm{nm}$ range (blue band), stronger than it is in the AA powders.

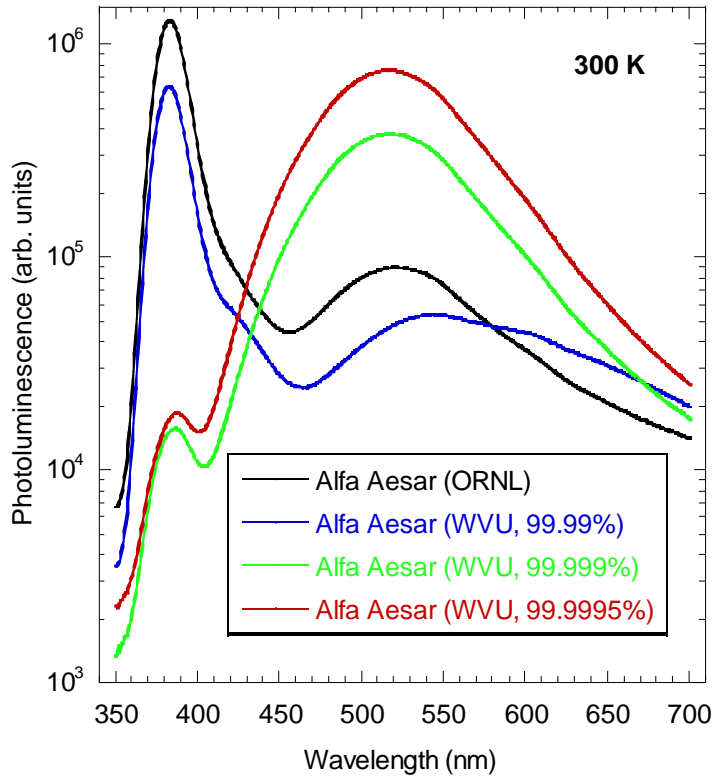

(a)

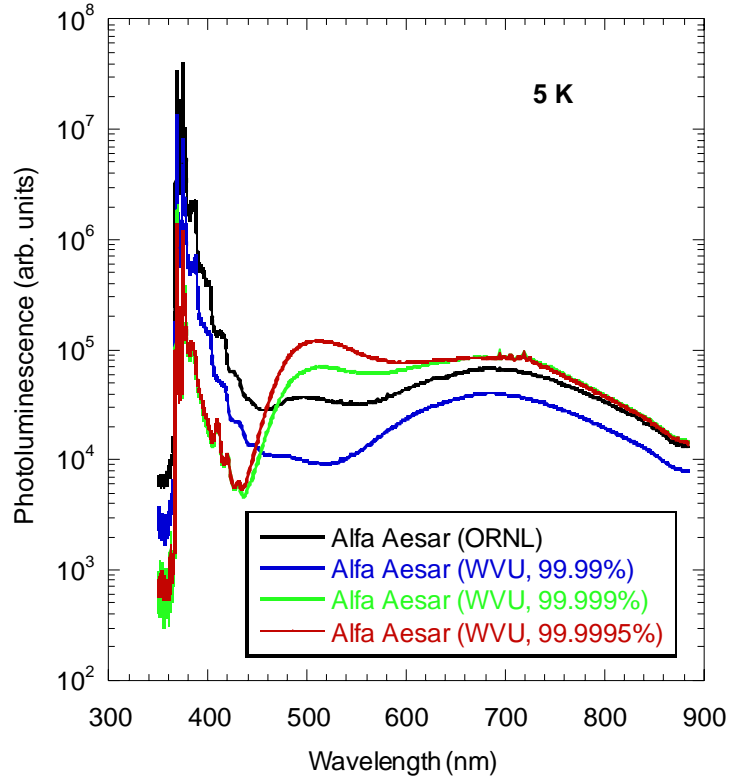

(b)

Figure 7.9 PL spectra taken at (a) $300 \mathrm{~K}$, and (b) $5 \mathrm{~K}$, from four Alfa Aesar $\mathrm{ZnO}$ powder samples purchased by WVU and ORNL. The purity level from the ORNL sample is unknown. Notice the logarithmic vertical scales.

Figure 7.9(a) shows PL spectra taken at $300 \mathrm{~K}$ from three AA samples purchased by WVU, with different purity levels, and one AA sample purchased by ORNL (purity unknown). It is striking that the two samples with higher purities show stronger visible emissions, with the "copper green" being dominant. The $99.99 \%$ pure sample has a spectrum more similar to those from the Sigma Aldrich samples: the green band shifts to $550 \mathrm{~nm}$, and both the yellow band and the blue band are clear. Figure 7.9(b) shows the PL spectra taken at $5 \mathrm{~K}$ from the same four samples. From all four samples, bound-exciton transitions and their phonon-replica lines dominate the edge emission range. The visible region contains a 
green band with peak at $500 \mathrm{~nm}$ and a red band at $700 \mathrm{~nm}$, similar to other samples already discussed. A series of PL lines with a zero-phonon-line at about $410 \mathrm{~nm}(3.02 \mathrm{eV})$ is assigned to DAP with accompanying phonon replicas. These lines evolve into the 400-450 $\mathrm{nm}$ "violet" band at room temperature (Fig. 7.9a). If one takes the 5-K bandgap energy as $\mathrm{E}_{\mathrm{g}}$ $=3.44 \mathrm{eV}$, the donor binding energy as $\mathrm{E}_{\mathrm{D}}=0.07 \mathrm{eV}$, and ignores the Coulomb and van de Waals interactions, the zero-phonon DAP line can be related to an acceptor with a binding energy $\mathrm{E}_{\mathrm{A}}=0.35 \mathrm{eV}$. The set of sharp lines observed from the two high purity samples in the range of $690-720 \mathrm{~nm}$ are related to $\mathrm{Fe}^{3+}$ ion impurities. These lines are attributed to the internal transitions from the ${ }^{4} \mathrm{~T}_{1}(\mathrm{G})$ state to the ${ }^{6} \mathrm{~A}_{1}(\mathrm{~S})$ state of $\mathrm{Fe}^{3+}$, with the zero-phonon line occurring at $694 \mathrm{~nm}(1.786 \mathrm{eV})(\mathrm{Heitz}, 1992)$.

\subsection{PL Characterization of Ceramic ZnO Samples}

The use of $\mathrm{ZnO}$ as a scintillator will require higher material density than can perhaps be achieved using a pressed powder. For the specific application of an alpha-particle detector at ORNL which motivated this work, fabrication of transparent ceramic samples with bright UV emission is the ultimate objective. Powders prepared at ORNL as well as those obtained from the commercial sources (AA and SA) were sintered using hot-uniaxial press (HUP) (by DeVito) and spark-plasma sintering (SPS) (By Hong).

In the HUP sintering technique (Neal, 2008), powder samples are loaded into a graphite-coated die with a $1.91 \mathrm{~cm}$ diameter cavity which is placed into the press, which is then evacuated to a vacuum of $200 \mathrm{mTorr}$ and held for at least 1 hour. A pressure of 28-35 MPa is applied to the press in one direction, while heating up to $900-1100{ }^{\circ} \mathrm{C}$. The rate of increase of both pressure and temperature are linear before they reach their desired values 
simultaneously. After holding at the final sintering conditions for 2 hours, the temperature is first lowered by about $50 \%$ before decreasing the pressure, to avoid the expansion of material upon sudden reduction of pressure.

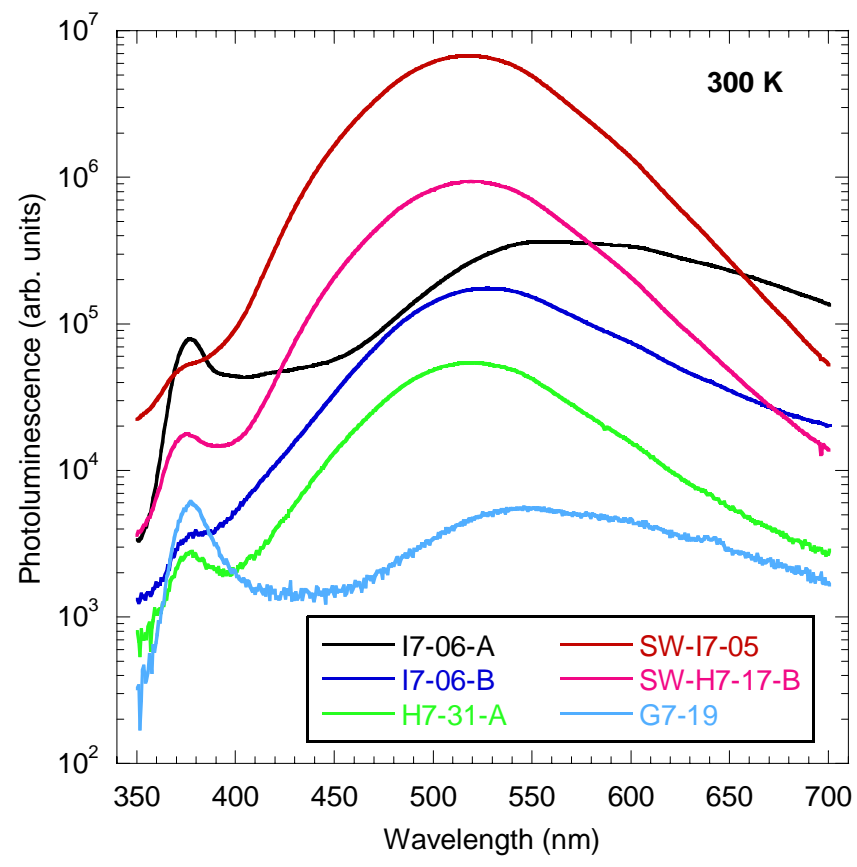

Figure 7.10 PL spectra taken at $300 \mathrm{~K}$ from 6 ceramic samples prepared using hot uniaxial pressing (HUP) technique. Notice the logarithmic vertical scale.

Figure 7.10 shows the PL spectra taken at $300 \mathrm{~K}$ from some ceramic samples

prepared by the HUP process. The samples were sintered at $1000-1200{ }^{\circ} \mathrm{C}$, under $1000-1050$ kg pressure for 2,5 , or 7 hours. Despite the variation in original powder and sintering conditions, most of the ceramic samples show bright green emissions. The brightest sample, SW-I7-05, was sintered at $1200{ }^{\circ} \mathrm{C}$ for 5 hours. The sample G7-19 has the best UV-to-visible intensity ratio. It was prepared using an AA powder and sintered under 1050-kg pressure at $1000{ }^{\circ} \mathrm{C}$ for 5 hours.

In the HUP sintering process, a long time period (e.g., several hours) is required during the application of pressure and high temperature. This causes significant grain-size increase. From the Raleigh scattering relation, the attenuation of due to grain boundary 
scattering is proportional to $\mathrm{f}^{4} \mathrm{~d}^{6}$, where $\mathrm{f}$ is frequency of the incident light, and $\mathrm{d}$ is the average grain size (Bohren, 1998). To realize fast sintering and better control over particle size, the SPS technique was employed at UC-Davis. In this technique (Hong, 2008), powders are first loaded into the graphite die and pressed under $8.9 \mathrm{kN}$ before heating. Heating is provided by a coil bearing a pulsed DC current (Sumitomo Coal Mining Co., Model 2050), with a working pattern of 12 consecutive pulses followed by 2 missing pulses. This provides a heating rate of about $200{ }^{\circ} \mathrm{C} / \mathrm{min}$. Once the target sintering temperature $\left(500-1100{ }^{\circ} \mathrm{C}\right)$ is reached, the temperature is held for 5-6 minutes under pressure of 50-700 MPa. The pressure is then rapidly released and heating is turned off. The whole sintering process takes about 10 minutes.

Figure 7.11 shows the 300-K PL spectra from four ceramic samples prepared using the SPS technique along with the PL spectrum taken from the starting AA powder. The starting powder was AA ZnO with $99.9995 \%$ purity (lot \# 23475). The sintering temperatures for these samples varied from $550{ }^{\circ} \mathrm{C}$ to $1050{ }^{\circ} \mathrm{C}$, while the holding pressure varies from $109 \mathrm{MPa}$ to $131 \mathrm{MPa}$. As shown earlier in this Chapter, the AA powders can have weak or strong UV emissions, depending on the particular lot number of the sample package. The starting powder for this group of ceramic samples has a bright UV emission and a moderate visible band intensity peaking near $530 \mathrm{~nm}$ (Fig. 7.11, curve a). SPS sintering at $550{ }^{\circ} \mathrm{C}$ under $111 \mathrm{MPa}$ produced a ceramic sample with a density of $3.93 \mathrm{~g} / \mathrm{cm}^{3}$, about $70 \%$ of the bulk crystal density. An SEM measurement found that the average grain size is $0.13 \pm 0.05$ micron in the ceramic sample. The UV emission from this sample is 100 times weaker than the starting powder, while the visible emission does not change much (Fig. 7.11, curve b). Sintering temperatures of $700-1050{ }^{\circ} \mathrm{C}$ produced ceramic samples with 
densities close to $100 \%$ of the bulk density, and larger grain sizes with mean values varying from 1.2 to 8.1 microns. The PL spectra all feature bright green emission and reduced UV emission (Fig. 7.11, curves c-e).

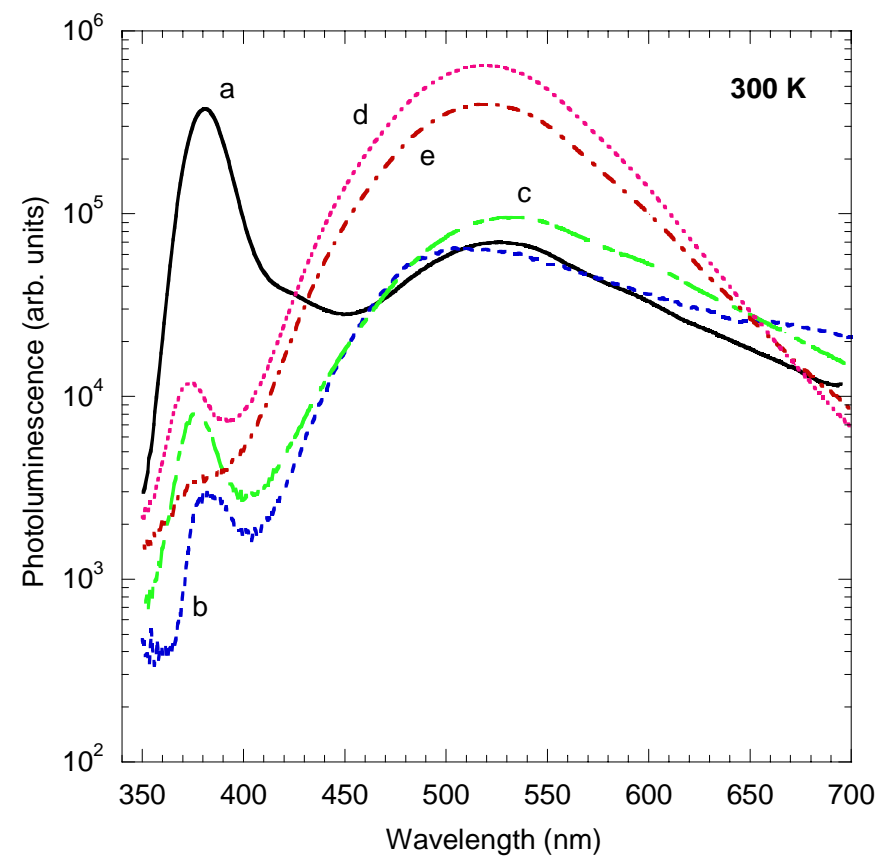

Figure 7.11 PL spectra from four ceramic samples at $300 \mathrm{~K}$, compared to the starting powder sample (a) Alfa Aesar lot\# 23475 (black curve). The ceramic samples were sintered using the SPS method at (b) $550{ }^{\circ} \mathrm{C}$, (c) 700 ${ }^{\circ} \mathrm{C}$, (d) $850{ }^{\circ} \mathrm{C}$, and (e) $1050{ }^{\circ} \mathrm{C}$. Note the logarithmic vertical scale.

To determine the origin of the strong green emission from these samples, ceramic samples prepared by SPS technique from the same AA powder and sintered at 550, 700, 950, and $1050{ }^{\circ} \mathrm{C}$, respectively, were subjected to air-anneals or Li-diffusion treatments to reduce the Fermi level. If the green emission is due to $\mathrm{Cu}$ impurities, then the characteristic structured $\mathrm{Cu}^{2+}$ emission (Dingle, 1969) should appear if the Fermi level is reduced and some neutral substitutional copper is present at thermal equilibrium. For air anneals, the ceramic samples were heated in air at $800{ }^{\circ} \mathrm{C}$ for 1 hour, and quickly cooled to room temperature in air. In the Li-diffusion treatment, one face of the ceramic sample is buried in LiF powder and heated at $800{ }^{\circ} \mathrm{C}$ in air for 1 hour. Figure 7.12 shows the PL spectra taken at $80 \mathrm{~K}$ in the range of $420-880 \mathrm{~nm}$ from the $950{ }^{\circ} \mathrm{C}$ sintered sample before and after the air- and the LiF- 
anneal treatments. The $80-\mathrm{K}$ PL from the as-prepared ceramic sample has an unstructured green band peaking at $510 \mathrm{~nm}$, and a red band at about $730 \mathrm{~nm}$. After annealing in air, the green band becomes much stronger and the peak shifts to about $525 \mathrm{~nm}$. The shortwavelength side of this green band has a step-like structure. The detection of the structured green band is evidence of $\mathrm{Cu}^{2+}$ ions in this sample (Dingle, 1969; Garces, 2002). The LiF diffusion produces substitutional $\mathrm{Li}$ acceptors in the ceramic sample. The $80-\mathrm{K}$ PL is dominated by a very strong yellow band peaking at about $610 \mathrm{~nm}$, but the step-like structure in the $420-530 \mathrm{~nm}$ range and the red band are still recognizable. The yellow band is the DAP band associated with a neutral shallow donor and neutral Li acceptor, and the red band is most likely related to $\mathrm{Zn}$ vacancies, as discussed in the previous section.

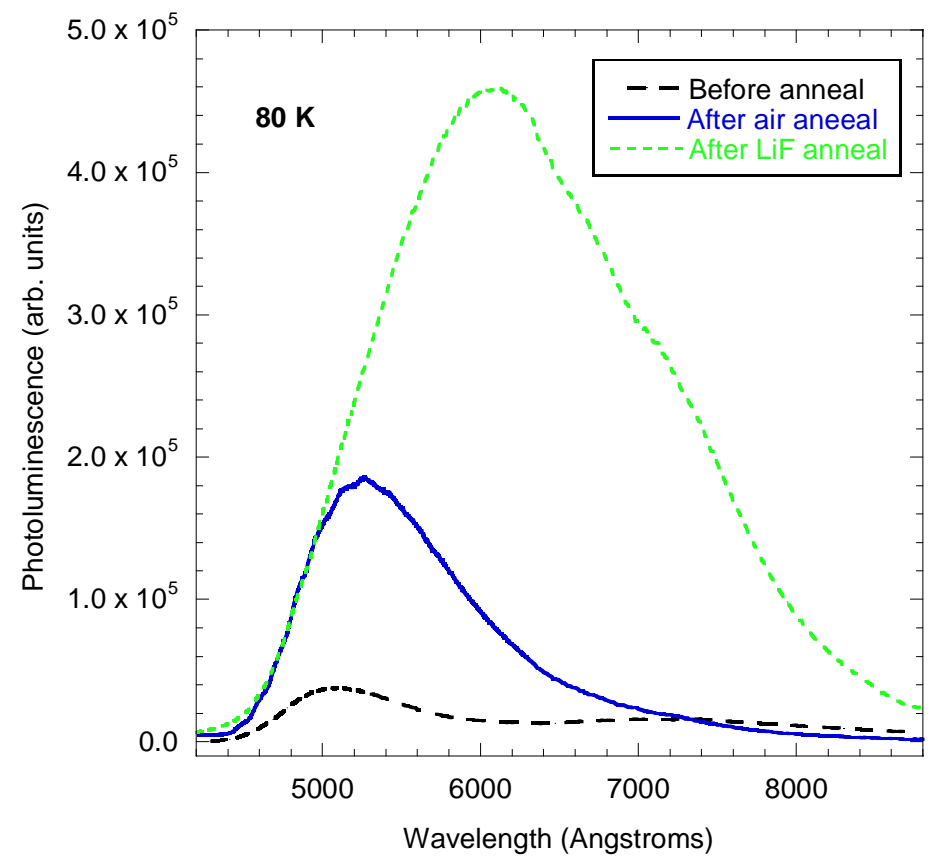

Figure 7. 12 PL spectra taken at 80 $\mathrm{K}$ from a ceramic sample sintered using SPS method at $950{ }^{\circ} \mathrm{C}$ with a pressure of $131 \mathrm{MPa}$. The air and $\mathrm{LiF}$ anneals were performed at 800 ${ }^{\circ} \mathrm{C}$ for 1 hour.

Figure 7.13 shows an expanded view of the $420-560 \mathrm{~nm}$ range from two SPS ceramic samples originally sintered at two different temperatures. The data were taken at $80 \mathrm{~K}$ after the samples were annealed in air. Both samples exhibit the structured green band due to $\mathrm{Cu}^{2+}$ 
ions. Curve (a) is from the $1050{ }^{\circ} \mathrm{C}(126 \mathrm{MPa})$ sintered ceramic, and curve (b) is from the $950{ }^{\circ} \mathrm{C}(131 \mathrm{MPa})$ sintered ceramic. Curve (c) is the PL spectra taken at $5 \mathrm{~K}$ from a SCVT single crystal $\mathrm{ZnO}$ sample after annealing in air at $900{ }^{\circ} \mathrm{C}$ for 1 hour. This latter curve shows the well-resolved $\mathrm{Cu}^{2+}$ emission in $\mathrm{ZnO}$, without interference from other longer-wavelength visible emission bands. The air anneal lowered the Fermi level in these samples, and at least a portion of the copper impurities are now in the $\mathrm{Cu}^{2+}$ state. Compared to the clear phononassisted structure of the 5-K spectrum from the single crystal sample (curve c), the $80-\mathrm{K}$ spectra from the two ceramics are most likely superposition of multiple components, the strongest being the structured green band described by Dingle (Dingle, 1969), and the unstructured green band. The latter requires that the initial state of copper is in the $\mathrm{Cu}^{+}$state, i.e., singly ionized acceptor. Upon laser excitation, the $\mathrm{Cu}^{+}$ion traps a free hole and becomes a neutral acceptor $\left(\mathrm{Cu}^{2+}\right)$, which in turn recombines with a neutral shallow donor and gives rise to the unstructured DAP green band (Garces, 2002).

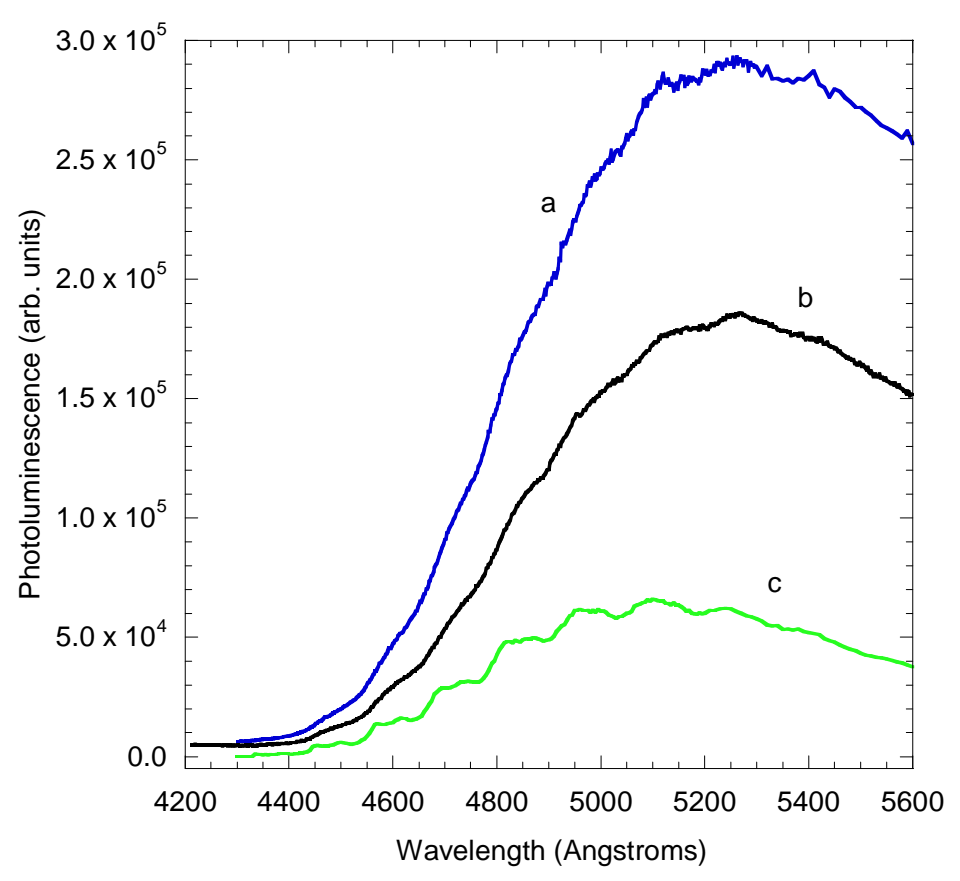

Figure 7. 13 PL spectra taken at 80 $\mathrm{K}$ from two SPS ceramic samples sintered at (a) $1050{ }^{\circ} \mathrm{C}$, and (b) 950 ${ }^{\circ} \mathrm{C}$, after the samples were annealed at $800{ }^{\circ} \mathrm{C}$ in air for 1 hour. Curve (c) shows the structured PL spectrum taken at $5 \mathrm{~K}$ from a single crystal $\mathrm{ZnO}$ sample that was annealed in air at $900{ }^{\circ} \mathrm{C}$ for 1 hour. 
Regardless of the starting powders (e.g., either synthesized at ORNL or purchased from commercial sources), PL spectra from the ceramic samples prepared from both HUP and SPS sintering methods show enhanced visible emissions and reduced UV emissions, quite opposite to the desired result. The strong visible emissions are mainly related to copper contamination, which most likely comes with the starting powder.

As shown in Section 7.1, H-anneal treatment on powder $\mathrm{ZnO}$ :Ga samples enhanced the UV emissions and reduced the visible emissions. This treatment is applied to some of the ceramic samples prepared using SPS sintering technique. The first sample is called WL1201, a ZnO:Ga powder prepared using wet chemistry method (Lehmann, 1966). The Ga doping level in this sample is $0.3 \mathrm{~mole} \%$. The as-prepared powder almost has no luminescence. After anneal in hydrogen gas at $700{ }^{\circ} \mathrm{C}$, it has a light blue color, and bright UV emission similar to some best commercial $\mathrm{ZnO}$ :Ga scintillator powders reported in another study (Neal, 2006). A ceramic sample was sintered from the hydrogen-annealed powder sample WL1201 using the SPS method at $950{ }^{\circ} \mathrm{C}$ under $116 \mathrm{MPa}$ for 5 minutes. A hydrogen anneal treatment was then performed on the ceramic sample. Figure 7.14 shows the PL spectra from the ceramic sample before and after hydrogen anneal, in comparison with that from the precursor powder sample WL1201. The UV emission from the as-prepared ceramic sample (curve a) is about 250 times weaker than the original powder (curve c), and the peak from the ceramic appears at about $8 \mathrm{~nm}$ shorter wavelength ( $66 \mathrm{meV}$ higher in energy) than from the powder. The PL spectrum taken from the powder using a different system (HR640) with higher resolution shows that besides the main peak at $390 \mathrm{~nm}$, there is a shoulder peak on the higher-energy side at about $377 \mathrm{~nm}$ (not shown). The shoulder band and the main peak are likely the FX and its second phonon replica, respectively. The sintering process seems to 
have eliminated most of the phonon-assisted transitions. After hydrogen anneal treatment of the ceramic sample, the UV intensity increased by a factor of about 50 times (curve b), making the ceramic a bright UV emitter comparable to some as-prepared single crystals. The peak position didn't change significantly after $\mathrm{H}$-anneal. The visible emission from the WL1201 powder is weak, and is also weak in the ceramics.

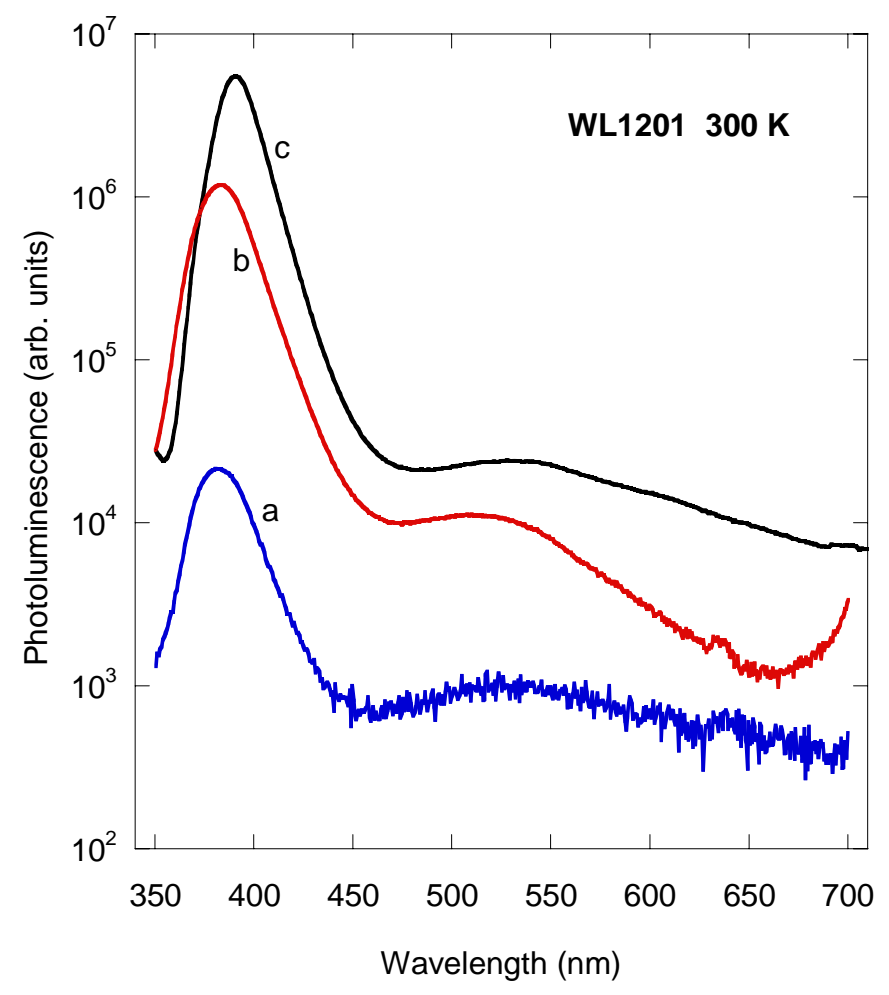

Figure 7.14 300-K PL taken from a ceramic sample before (a) and after (b) hydrogen anneal treatment. The sample was prepared using SPS method from the WL1201 powder (c). Notice the logarithmic vertical scale.

A similar treatment was performed on a ceramic sample sintered from powder ZnO:Ga sample BK5-203. The powder sample was prepared using solution synthesis at ORNL. The as-prepared sample has particle size ranging from 15 to $20 \mathrm{~nm}$, with agglomerates ranging from $200 \mathrm{~nm}$ to several microns. The powder sample was completely consumed in preparation of ceramics and before a PL characterization was able to be done on the starting powder. The ceramic sample was sintered using SPS method at $1050{ }^{\circ} \mathrm{C}$ under $437 \mathrm{MPa}$ for 5 minutes. The product was not as robust as the WL1201 ceramic, and is very 
easy to delaminate. Figure 7.15 shows the $300-\mathrm{K}$ PL from the as-prepared BK5-203 ceramic (curve a) and after the hydrogen anneal (curve b). The as-prepared ceramic has moderate UV emission. The visible band peaking at about $520 \mathrm{~nm}$ has intensity a little higher than that of the UV edge emission. After the hydrogen anneal, both uv and visible intensities increased by more than 10 time, but the UV band is now brighter than the green band.

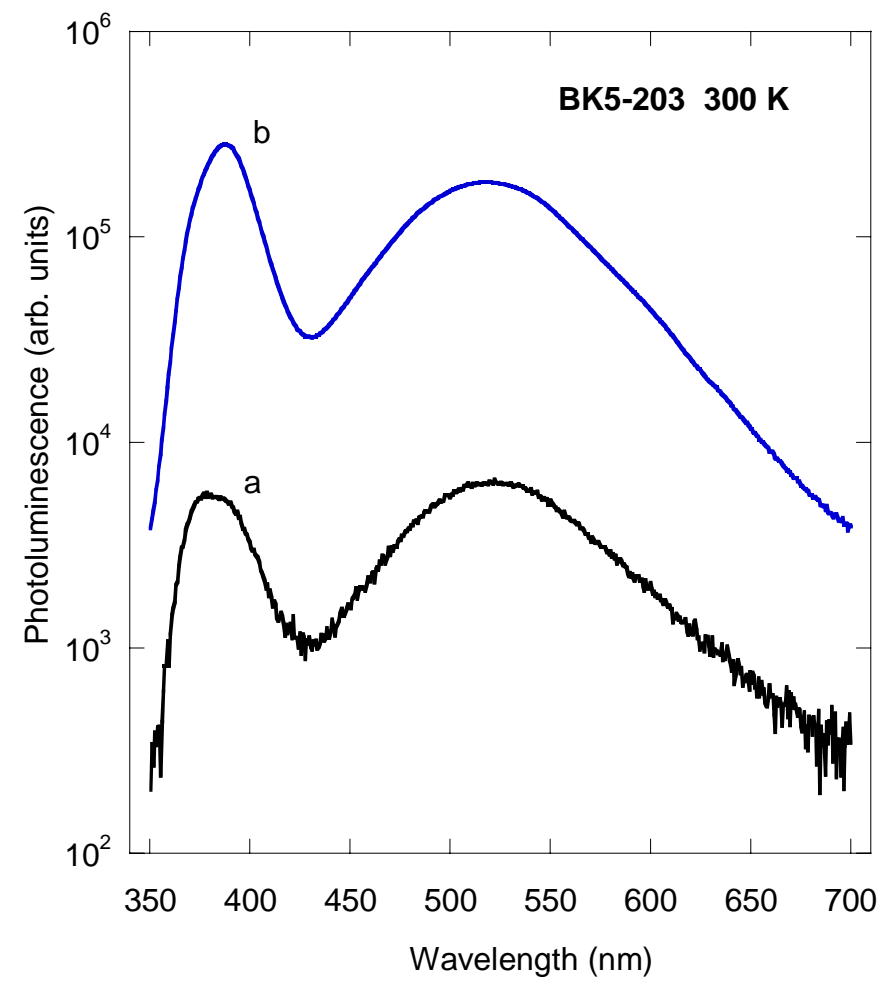

Figure 7.15 300-K PL taken from ceramic sample BK5-203 before (a) and after (b) hydrogen anneal treatment. Notice the logarithmic vertical scale.

The effects of hydrogen anneal treatments on ceramic samples prepared from commercial $\mathrm{ZnO}$ powders were also explored in this study. Figure 7.14 shows the PL spectra from a SPS-sintered ceramic sample prepared from Alfa Aesar ZnO powder (lot\# 23558). The as prepared ceramic has moderate visible (green) emission and very weak UV emission (curve a), both about two decades weaker than the original powder (curve c). After the Hanneal treatment, the intensities of both the edge and visible emissions increased by three orders (curve b), but the uv-to-visible intensity ratio stayed the same. This result indicates 
that the $\mathrm{H}$-anneal treatment on ceramics prepared from undoped $\mathrm{ZnO}$ powders will not be helpful in improving the UV emission. The commercial powders contain impurities/defects which produce bright visible emission.

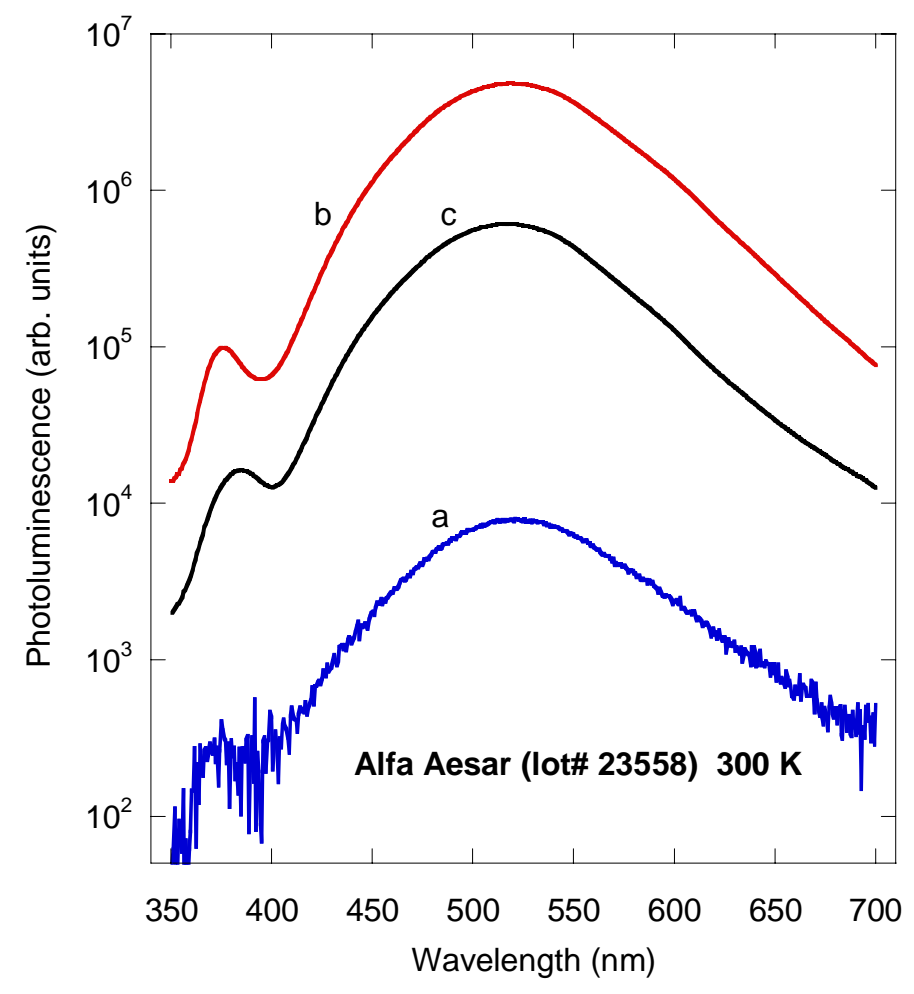

Figure 7.16 300-K PL from a SPS sintered ceramic sample before (a) and after (b) H-anneal. The original powder is Alfa Aesar $\mathrm{ZnO}$ (lot\# 23558 ) and its $300-\mathrm{K}$ PL is shown as curve (c). Notice the logarithmic vertical scale.

\subsection{X-Ray-Induced Luminescence of $\mathrm{ZnO}$ and ZnO:Ga Samples}

Scintillator applications often include high energy excitations such as cosmic rays and radiative beams. For the associated alpha particle detector used in the D-T neutron generator at ORNL, the neutron beam energy is $14.1 \mathrm{MeV}$, hence the energy of the recoil alpha particles could be about $3.5 \mathrm{MeV}$, which is much higher than the photon excitation energy corresponding to the $325-\mathrm{nm}$ laser $(3.81 \mathrm{eV})$ used in the PL measurements. To evaluate the luminescent behavior of $\mathrm{ZnO}$ samples used in such scintillation environments, $\mathrm{X}$-ray-induced luminescence is applied in addition to the PL characterization. As described in Chapter 3 (Section 3.3), the X-ray excitation is provided by a tungsten X-ray tube, operating at $30 \mathrm{~mA}$ 
and $60 \mathrm{kV}$. The luminescence emission is collected either in transmission or reflection geometry by a fiber head, and transferred through the fiber to a spectrograph system (Oriel, MS260i) CCD detector for analysis.

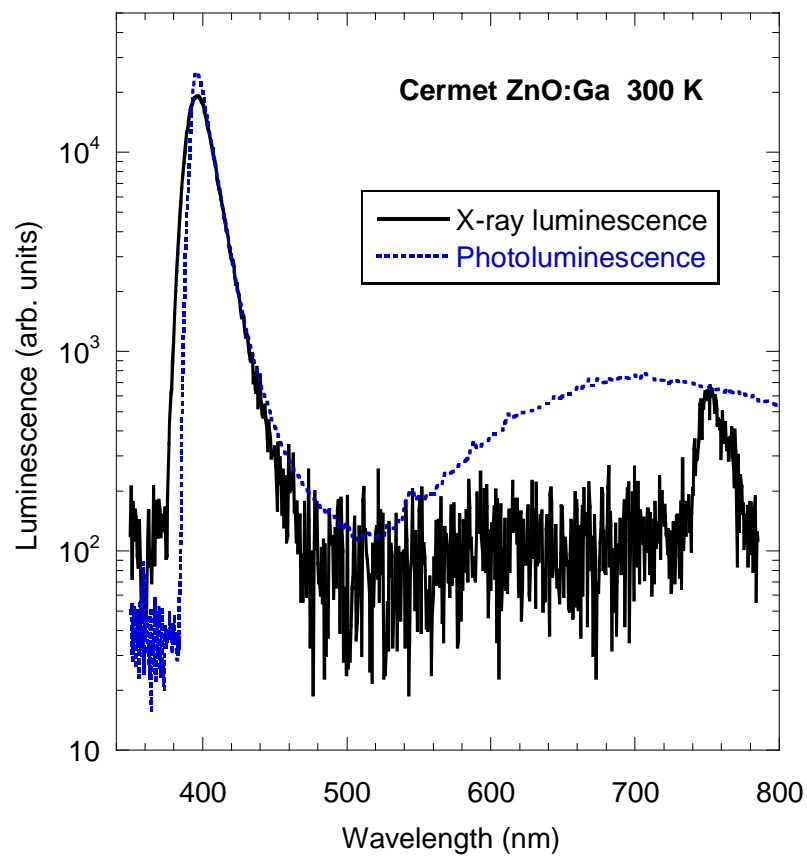

Figure 7.17 X-ray-induced luminescence (solid line) from single crystal $\mathrm{ZnO}: \mathrm{Ga}$ (Cermet) sample compared with its PL spectrum (dashed line). Both spectra were collected using transmission geometry. Notice the logarithmic vertical scale.

Figure 7.17 shows the X-ray induced luminescence spectrum (solid line) from the heavily doped $\mathrm{ZnO}$ :Ga single crystal (Cermet, $\mathrm{n}=2 \times 10^{19} \mathrm{~cm}^{-3}$ ) that was discussed in Chapter 4. The PL spectrum excited by $325 \mathrm{~nm}$ laser is also presented for comparison (dashed line), which was taken using the monochromator (Instrument SA, Inc. HR-640)/PMT (Hamamatsu R943-02) detector system. Both spectra shown in Figure 7.17 are collected using transmission geometry. The PL spectrum of this sample has a moderate UV peak, about 6 times dimmer than the UV emission collected using reflective geometry. The broad visible emission has a peak at about $700 \mathrm{~nm}$, and its intensity is about 30 times weaker than the UV peak intensity. The X-ray induced luminescence is similar to the PL spectrum in the UV edge emission. The broadening of the UV band is most likely due to the lower resolution 
of the CCD detector system. The broad visible band in PL is not seen in X-ray luminescence, which is good for scintillation application. The narrow band in the $740-800 \mathrm{~nm}$ range is the second order diffraction of the edge emission band. This band is eliminated in the PL spectrum because a long-pass filter was placed in the optical path in front of the monochromator for collection of luminescence having wavelengths greater than $620 \mathrm{~nm}$.

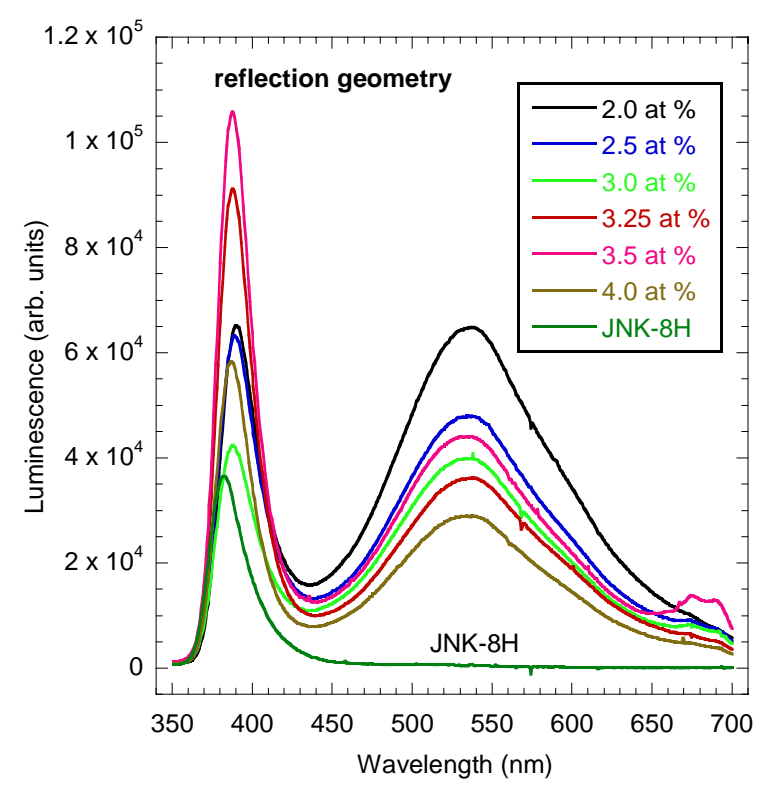

(a)

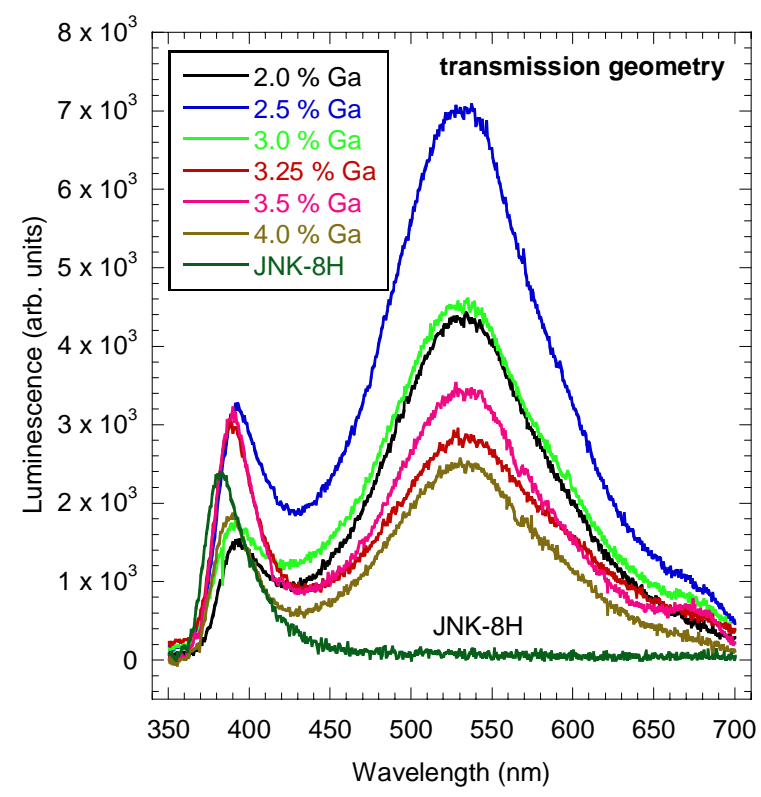

(b)

Figure 7.18 X-ray-induced luminescence from six solid-state synthesized $\mathrm{ZnO}$ :Ga powder samples collected using (a) reflective and (b) transmission geometries. The results from the solution-phase synthesized sample JNK-8H are used for comparison.

Powder $\mathrm{ZnO}$ :Ga samples prepared at ORNL by solid state synthesis were characterized using X-ray-induced luminescence in both reflective and transmission geometries. These samples have been doped with $2-4$ at \% gallium. Figure 7.18 shows their $\mathrm{X}$-ray luminescence in comparison with sample $\mathrm{JNK}-8 \mathrm{H}$, which is synthesized using solution phase method and is doped with 15,000 ppm gallium. The reflective luminescence spectra (Figure 7.18, a) show both bright edge emission and visible emission. The UV edge 
emissions from the solid-state synthesized samples are brighter than that from JNK-8H. This is different than the PL comparison. As shown earlier in Fig. 7.7, the UV PL emission from these samples is weaker than that from JNK-8H. Particle size may play an important role in the sample-to-sample comparison in Fig. 7.18a, since the scattering effect will affect the absorption of X-ray and the consequent luminescence. However, as in the PL comparison, the "winner" of best UV-to-visible intensity ratio (as indicated by these optical measurements) is still the sample JNK-8H. The transmission luminescence spectra (Fig. 7.18, b) show a drastic decrease in intensity for both edge and visible emissions, believed to be mainly due to particle-size-dependent scattering, and the UV emission is reduced more than the visible emission. Assuming a simple Rayleigh scattering model, the scattering loss of light is proportional to the fourth powder of frequency. Along the path through the powder sample, the scattering loss for the UV emission peak ( $390 \mathrm{~nm}$ ) is about 3.4 times larger than that for the visible peak $(530 \mathrm{~nm})$.

Figure 7.19 shows a comparison of X-ray luminescence (transmission geometry) with PL (reflection geometry) from two commercial powder $\mathrm{ZnO}$ samples. The two samples were purchased from Alfa Aesar (AA, lot\# unknown) and Sigma Aldrich (SA, 99.999\% pure). The X-ray luminescence from both sample AA (curve a) and SA (curve b) show moderate UV emission dominated by a more intense broad visible emission. In the PL spectra (reflection geometry), it is the UV emission that dominates in both AA (curve c) and SA (curve d) samples. Comparison of the PL and X-ray luminescence spectra shows that longer wavelengths are favored in the transmission $\mathrm{x}$-ray luminescence, which confirms that scattering loss is a key factor to explain the intensity decrease of the fast edge emission. 


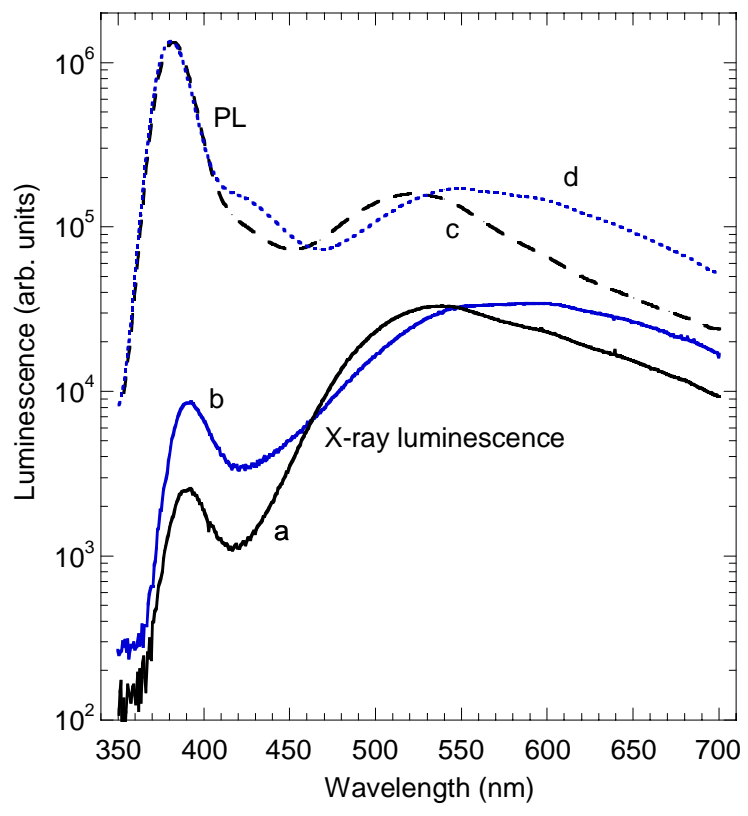

Figure 7.19 X-ray-induced luminescence (transmission geometry) from commercial $\mathrm{ZnO}$ powder samples (a) Alfa Aesar from ORNL (AA, lot\# unknown), and (b) Sigma Aldrich (SA, 99.999\% pure), in comparison with their PL spectra (reflection geometry): (c) from AA and (d) from SA. Notice the logarithmic vertical scale.

For those "best" $\mathrm{ZnO}$ :Ga powders that demonstrated bright UV PL emission and very weak visible emissions, X-ray luminescence shows that they are still the most promising. Besides the WL1201 powder prepared by W. Lehmann and described earlier in this chapter, three other $\mathrm{ZnO}$ :Ga powder samples are also characterized using both PL and X-ray luminescence here. One of them is sample LBNL3518, which is prepared at Lawrence Berkeley National Laboratory (LBNL) using solid-state reaction, doped with 0.15 mole $\%$ of gallium and subjected to hydrogen anneal (Neal, 2006). The other two ZnO:Ga powders were purchased by ORNL from two commercial sources, NETech (Nuclear Enterprises Technology) and GK31 (Phosphor Technologies), and were also annealed in hydrogen. A laser scattering particle size analyzer (Horiba) was used and the amediam particle sizes were found to be 8.2 (LBNL3518), 5.1 (WL1201), and 0.9 micron (NETech and GK31) (Neal, 2006). PL spectra from the four powder samples are shown in Figure 7.20(a). The brightest UV emission is from sample GK31, which also has a broad visible band. Another commercial powder, NETech, has a UV peak less bright than the other three samples, and a 
green band peaking at about $510 \mathrm{~nm}$. The LBNL3518 sample has UV emission as bright as that of sample WL1201, but its visible emission is even weaker, which makes it a better scintillator material than the latter. The sample-to-sample comparison in the UV emission of X-ray induced luminescence from these samples has a different pattern. As shown in Figure 7.20 (b), X-ray luminescence from all four samples show bright UV peak dominating the visible bands. Samples LBNL3518 and WL1201 are the winners for both brighter UV emission and higher UV/visible intensity ratio. The two commercial powders have broad visible emissions with some sharp lines superimposed on them. The origin of these sharp lines is not clear. They might be impurity-related transitions that can be excited by X-ray, but not by the $325-\mathrm{nm}$ laser.

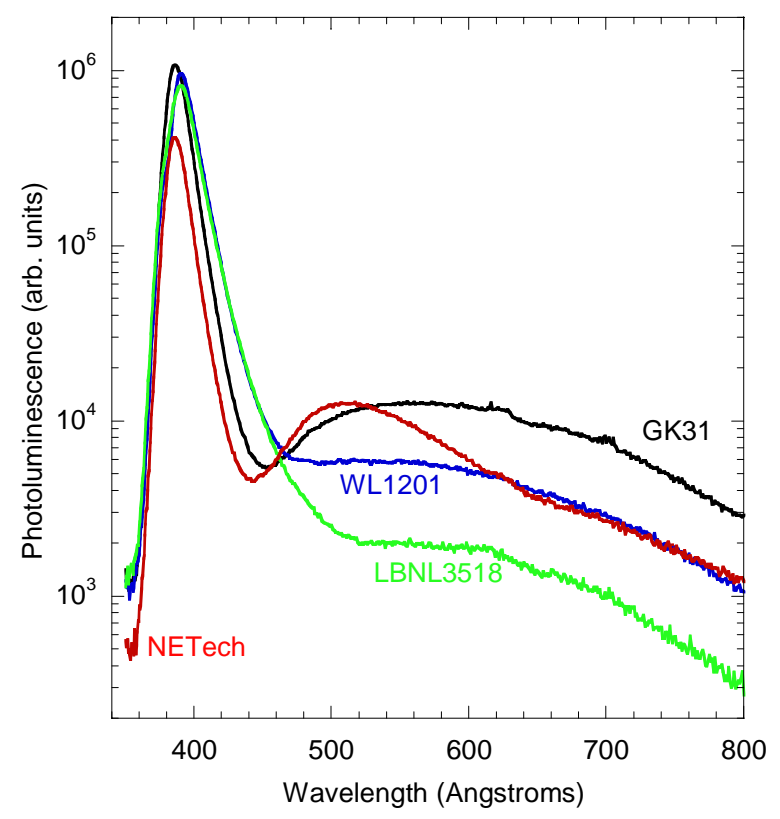

(a)

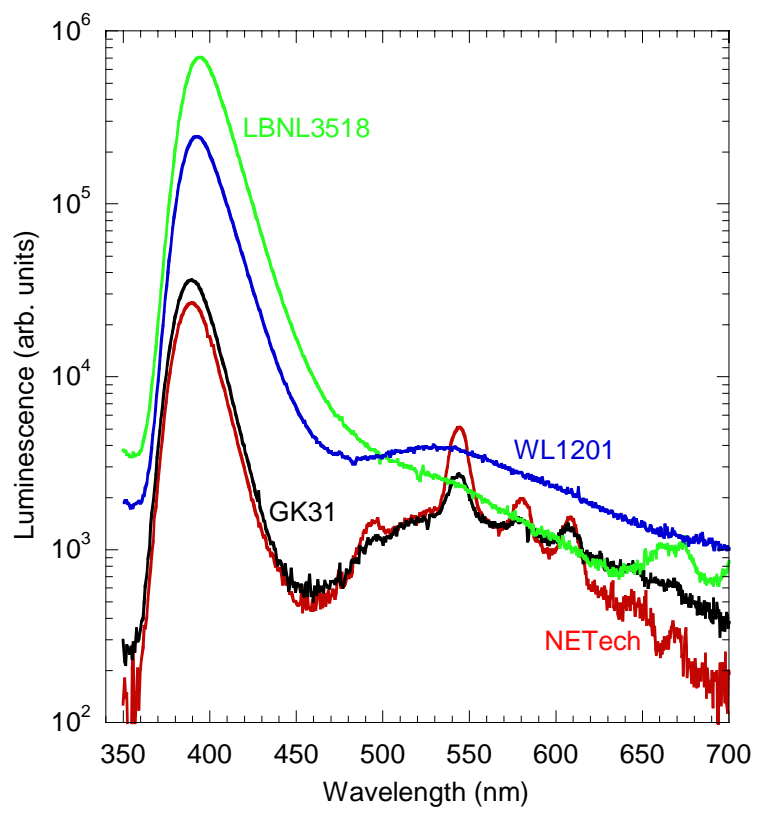

(b)

Figure 7.20 PL spectra (a) and X-ray-induced luminescence spectra (b) from four $\mathrm{ZnO}: \mathrm{Ga}$ powders collected using transmission geometry. Notice the logarithmic vertical scale.

X-ray luminescence from ceramic samples prepared using the HUP and SPS sintering techniques with $\mathrm{ZnO}$ powders either synthesized at ORNL or obtained commercially show 
similar emission spectra. The visible emission peaking around $530 \mathrm{~nm}$ is the dominant feature and the UV emission is either very weak or not detected. Luminescence collected in reflective geometry from some of the ORNL synthesized sample show weak to moderate UV peaks, but none of them will survive in the transmission geometry. Figure 7.21 shows the Xray induced luminescence collected in transmission geometry from a ceramic sample (Pure950), before and after hydrogen anneal treatment. The sample was sintered with AA $\mathrm{ZnO}$ powder (99.9995\%) using SPS method at $950{ }^{\circ} \mathrm{C}$ under $131 \mathrm{MPA}$ for 5 min. X-ray luminescence from the as-prepared ceramic has a very bright green emission and almost no UV emission peak (curve a). After the $\mathrm{H}$-anneal treatment, the visible emission becomes even stronger, but there is still no edge emission peak (curve b).

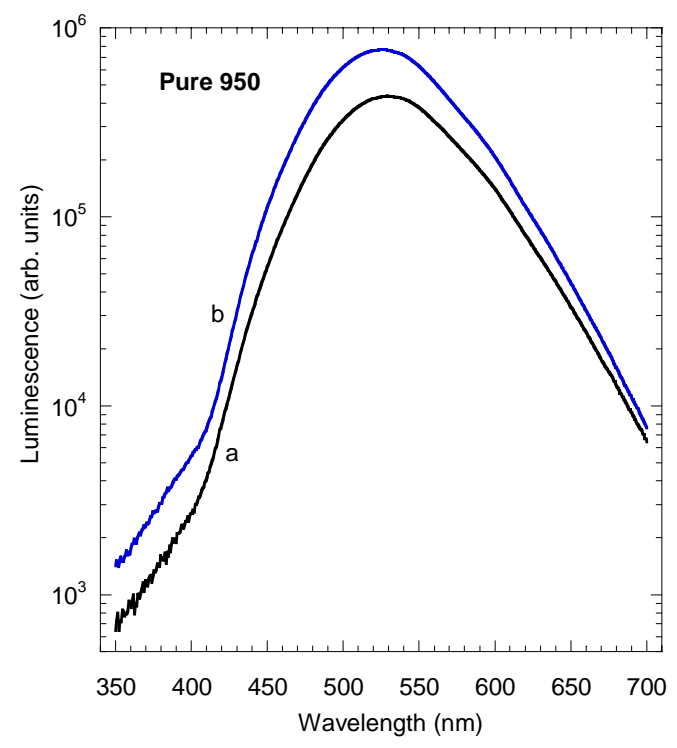

Figure 7.21 X-ray induced luminescence collected using transmission geometry from ceramic sample Pure950: (a) as prepared, and (b) after H-anneal. Notice the logarithmic vertical scale.

Figure 7.22 shows the X-ray induced luminescence collected in transmission geometry from ceramic sample prepared using SPS sintering from the WL1201 powder, before and after a hydrogen anneal treatment. The PL spectra before and after $\mathrm{H}$-anneal have been discussed in the previous section and shown in Figure 7.14. The X-ray luminescence from the as-prepared WL1201 ceramic sample has a weak UV emission peak at about 390 
nm (curve a) that survives the scattering. No visible emission is detected, similar to the PL from this sample. The hydrogen anneal of the ceramic sample improved the UV emission by about a factor of 3 (curve b).

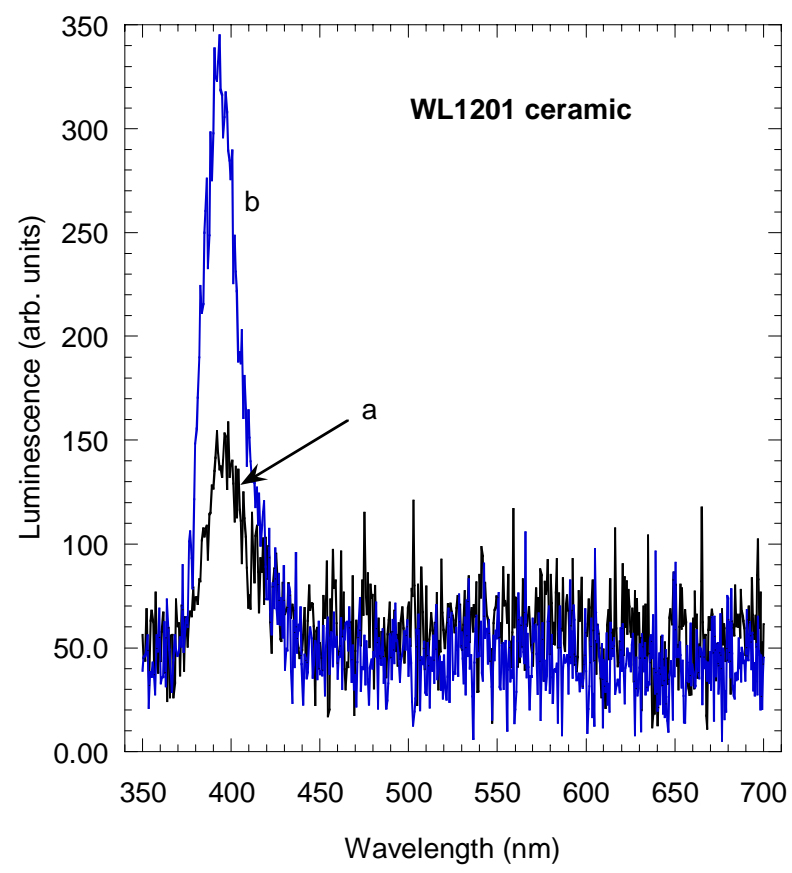

Figure 7.22 X-ray induced luminescence (transmission geometry) from ceramic sample WL1201, (a) before and (b) after H-anneal.

In summary, PL and X-ray induced luminescence characterizations were used to evaluate $\mathrm{ZnO}$ and $\mathrm{ZnO}: \mathrm{Ga}$ samples for scintillator applications. Single crystal samples, powder samples synthesized at ORNL and LBNL or purchased from commercial sources, and ceramic samples prepared using HUP and SPS sintering techniques, have been studied. Both PL and X-ray luminescence studies indicate that heavy doping with Ga (0.05-4.0 at \%), plus a hydrogen-anneal treatment (both on powder and ceramic samples) produces bright ZnO:Ga UV scintillators. Impurity (e.g., $\mathrm{Cu}$ ) content has important effect on preparation of ceramic scintillators using commercial $\mathrm{ZnO}$ powders. Particle size control is crytical since scattering loss is a major concern in transmission PL and X-ray induced luminescence. 


\section{Chapter 8 Summary}

Characterization tools including PL, X-ray-induced luminescence, Hall Effect, and optical absorption are employed in this work to study the electrical and optical properties of $\mathrm{ZnO}$ for use as an inorganic scintillator. The direct motivation is the application of powder and ceramic forms of $\mathrm{ZnO}: \mathrm{Ga}$ in an associated alpha-particle detector for the $\mathrm{D}-\mathrm{T}$ neutron generator at ORNL. Single crystal samples from three different growth techniques were studied to establish knowledge of the PL recombination mechanisms and the relation between PL emission and defects. Knowledge gained from single crystals was applied to understand the emission behavior of powder and ceramic samples fabricated at ORNL and purchased from commercial sources, using both PL and X-ray induced luminescence characterizations. The following is a summary of the main results of this study.

Room-temperature PL emissions from single crystals have different origins, depending on the growth technique used and the free-carrier level. Free-carrier-related bandgap renormalization including the effects of electron-ionized-impurity interaction, exchange interaction, and band-filling was considered in calculating the $300-\mathrm{K}$ band-gap energy for asgrown undoped and Ga-doped $\mathrm{ZnO}$ samples grown at Cermet. At $300 \mathrm{~K}$, the band-gap energy $\mathrm{E}_{\mathrm{g}}=3.353 \mathrm{eV}$ for the as-grown sample and $\mathrm{E}_{\mathrm{g}}=3.213 \mathrm{eV}$ for the $\mathrm{ZnO}$ :Ga sample were calculated. Line-shape analysis was performed to fit the measured PL spectra. Based on these analyses, the edge emission from the as grown Cermet sample is mainly due to FX and FX-1LO transitions, while that from the Ga-doped sample is mainly due to the direct band-to-band (e, h) recombination. 
The effect of self-absorption on PL was studied on three single crystal samples through comparison of 300-K PL spectra collected using transmission geometry with spectra taken using reflection geometry. Absorption coefficient data were used to correct the PL data and the origins of the survived edge emissions were explained. For the as-grown $\mathrm{ZnO}$ samples, emission from the two-phonon assisted transition (FX-2LO) escaped the selfabsorption. For the heavily doped Cermet $\mathrm{ZnO}$ :Ga sample, the FX emission is the survivor.

Temperature dependent PL taken from 5 to $300 \mathrm{~K}$ from as-grown $\mathrm{ZnO}$ confirmed the assignment of the room temperature luminescence to FX transitions. The 5-K PL from the as-grown sample has intense DBX lines associated with group III donors. As temperature increases, the DBX lines quench and the FX and its phonon replicas become dominant. The FX energy was used to fit the temperature-dependent band-gap using Manoogian-Wooley equation after the free carrier effects were taken into account, yielding an Einstein temperature $\theta_{\mathrm{E}}=750 \mathrm{~K}$. The value agrees with the effective temperature determined by the average energy of the top four optical phonons for $\mathrm{ZnO}$. On the other hand, the broad PL peak at $5 \mathrm{~K}$ from the heavily doped $\mathrm{ZnO}: \mathrm{Ga}$ sample makes it difficult to distinguish the contribution of $(\mathrm{e}, \mathrm{h})$ transition from FX recombinations.

Temperature-dependent intrinsic mobility and Hall $\mathrm{r}$ factor for $\mathrm{n}$-type $\mathrm{ZnO}$ single crystals were established by applying the relaxation time approximation (RTA) including three phonon scattering mechanisms. Established expressions were adopted, but some key parameters in these expressions were carefully chosen, such as the deformation potential $\left(E_{1}\right)$, piezoelectric coupling coefficient $(\mathrm{P})$, optical phonon effective temperature $\left(\mathrm{T}_{\mathrm{po}}\right)$, and effective electron polaron mass $\left(\mathrm{m}_{\mathrm{p}}{ }^{*}\right)$. The predicted intrinsic Hall mobility for n-type $\mathrm{ZnO}$ 
is $220 \mathrm{~cm}^{2} / \mathrm{Vs}$ at $300 \mathrm{~K}$ and $2850 \mathrm{~cm}^{2} / \mathrm{Vs}$ at $60 \mathrm{~K}$. Using the same approach, the intrinsic hole Hall mobility at $300 \mathrm{~K}$ is predicted to be $80 \mathrm{~cm}^{2} / \mathrm{Vs}$.

Measured temperature-dependent Hall mobility data from single-crystal samples by different growth techniques were compared to the intrinsic prediction. From RTA fitting by including the ionized and neutral impurity scatterings, donor and acceptor concentrations were obtained. Fitting of the temperature-dependent free carrier data after correction using Hall $r$ factors revealed the energy levels and concentrations of donors that contribute to free carriers. Origins of the donors and acceptors from these samples were identified through optical studies including liquid He temperature PL and absorption. Substitutional group-III shallow donors contribute to the free carriers and also serve as scattering centers in the SCVT and HPM grown samples. For the HYD grown samples, lithium acceptors exist in $10^{16}$ to $10^{17} \mathrm{~cm}^{-3}$, but are compensated by donors. Deep-level transition-metal-ion donors and $\mathrm{LiOH}^{-}$complexes serve as neutral scattering centers.

High n-type single-crystal $\mathrm{ZnO}$ prepared by extrinsic doping and anneal treatments were studied through optical absorption, PL, and Hall measurements. While extensive doping and co-doping produced some bright UV emitters with $\mathrm{n}$ in the high $10^{18} \mathrm{~cm}^{-3}$ to low $10^{19} \mathrm{~cm}^{-3}$, values of $\mathrm{n}$ higher than the Mott density from Ga-Li co-doping leads to lower emission efficiency. Absorption and reflectance spectra were used to estimate free carrier concentrations for the large-dimension samples. Post-growth anneal treatments of HPM- and HYD-grown $\mathrm{ZnO}$ increases $\mathrm{n}$ to the range of high $10^{17} \mathrm{~cm}^{-3}$ to low $10^{18} \mathrm{~cm}^{-3}$, but selfabsorption effect due to a near-edge absorption band peaking at $3.03 \mathrm{eV}$ limits the $\mathrm{UV}$ luminescence. The coloration of single crystal samples associated with doping or heat treatments was explained by UV/visible-IR absorption. The blue color from Ga-doped and 
co-doped Cermet samples was due to free carrier absorption that extends into the visible (red to orange) region. The red color from samples heat-annealed in reducing atmosphere is attributed to a localized absorption of oxygen vacancies produced or increased during the after-growth treatments.

Evaluation of powder and ceramic $\mathrm{ZnO}$ and $\mathrm{ZnO}: \mathrm{Ga}$ samples for scintillator application was performed using PL and X-ray induced luminescence characterizations. Powder samples were synthesized at ORNL and LBNL, or purchased from commercial sources. The brightest UV emitters are $\mathrm{ZnO}$ :Ga powders after hydrogen anneal treatments, such as samples WL1201, LBNL3518, NeTech, GK31, and JNK-8H. From study of ZnO:Ga powder samples prepared at ORNL using different synthesis techniques, hydrogen anneals appear to be efficient in passivation of the visible DAP emission related to Li acceptors, and help to improve the fast UV emission needed for scintillator application.

Ceramic samples were prepared using HUP and SPS sintering techniques from differing synthesis methods and venders of commercial powders. Compared to the starting powders, the ceramics show stronger visible emissions and reduced UV emissions. Anneal treatments in air and $\mathrm{LiF}$ helped attribute the commonly seen green emissions from ceramic samples to copper contaminants existing in the starting powders.

X-ray induced luminescence was used to simulate the harsh radiation environment of scintillators. The luminescence was collected using either reflective geometry or transmission geometry. The reflection geometry luminescence resembles the PL spectra for most samples, while the transmission geometry luminescence shows drastic decrease in the UV emissions. Scattering loss is blamed for this decrease, which becomes more severe as the powders are sintered into ceramics and experience significant grain size growth. 
In summary, n-type $\mathrm{ZnO}: \mathrm{Ga}$ single-crystal and powder samples make good UV scintillators, although the recombination mechanisms can differ from sample to sample. The synthesis methods, Ga doping levels, and after-synthesis treatments still need optimization. Contaminants such as $\mathrm{Cu}$ and $\mathrm{Li}$ from both "homemade" and commercially obtained powders are obstacles for brighter $\mathrm{ZnO}$ scintillators. For fabrication of high efficient ceramic scintillators, the choice of starting powders and sintering methods, particle-size control through sintering conditions such as temperature and pressure, and after-sintering treatments, present challenges as well as opportunities for future study. 


\section{References}

K. A. Alim, V. A. Fonoberov, M. Shamsa, and A. A. Balandin, J. Appl. Phys. 97, 124313(2005)

C. A. Arguello, D. L. Rousseau, and S. P. S. Porto, Phys. Rev. 181, 1351 (1969)

N. Ashkenov, B. N. Mbenkum, C. Bundesmann, V. Riede, M. Lorenz, D. Spemann, E. M. Kaidashev, A. Kasic, M. Schubert, M. Grundmann, G. Wagner, H. Neumann, V. darakchieva, H. Arwin, and B. Monemar, J. Appl. Phys. 93, 126 (2003)

W. S. Baer, Phys. Rev. 154, 785 (1967)

L. Bai, C. Xu, K. Nagashio, C. Yang, R. S. Feigelson, P. G. Schunemann, and N. C. Giles, J. Phys.: Condens. Matter 17, 5687 (2005)

T. B. Bateman, J. Appl. Phys. 33, 3309(1962)

H. B. Bebb and E. W. Williams, in Semiconductors and Semimetals, edited by R. K. Willardson and A. C. Beer, (Academic, New York, 1972), Vol 8, pp. 289-295

J. M. Bian, X. M. Li, X. D. Gao, W. D. Yu, and L. D. Chen, Appl. Phys. Lett. 84, 541 (2004)

J. S. Blakemore, Semiconductor Statistics (Dover, New York, 1987) Chapter 3

S. Bloom and I. Ortenburger, Phys. Status Solidi B 58, 561 (1973)

J. Boone and G. Cantwell, J. Appl. Phys. 57, 1171(1985)

C. F. Bohren, and D. R. Huffman, Absorption and scattering of light by small particles, (Wiley, New York, 1998)

M. Bugajski and W. Lewandowski, J. Appl. Phys. 57(2), 521 (1985)

S. C. Bushong, Radiologic Science for Technologists, (Mosby, St. Louis, MO, 1975)

P. F. Carcia, R. S. McLean, M. H. Reilly, and G. Nunes, Appl. Phys. Lett. 82, 1117 (2003)

Y. Chen, D. M. Bagnell, H.-J., Koh, K.-T., Park, K. Hiraga, Z.-Q. Zhu, and T. Yao, J. Appl. Phys. 84, 3912 (1998)

R. Chwang, B. J. Smith, and C. R. Crowell, Solid-State Electron. 17, 1217 (1974)

R. T. Cox, D. Block, A. Hervé, R. Picard, C. Santier, and R. Helbig, Solid State Commun. 25, $77(1978)$ 
W. Crookes, Certain Properties of the Emanations of Radium. Chemical News; Vol. 87241 (1903)

S. E. Derenzo, M. J. Weber, E. Bourret-Courchesne, and M. K. Klintenberg, Nuclear Instruments and Methods in Physics Research A 505, 111 (2003)

S. E. Derenzo, M. J. Weber, and M. K. Klintenberg, Nuclear Instruments and Methods in Physics Research A 486, 214 (2002)

R. E. Dietz, H. Kamimura, M. D. Sturge, and A. Yariv, Phys. Rev. 132, 1559 (1963)

R. Dingle, Phys. Rev. Lett. 23, 579 (1969)

R. Dinges, D. Frohlich, B. Staginnus, and W. Staude, Phys. Rev. Lett. 25, 922 (1970)

D. Ehrentraut, H.Sato, Y. Kagamitani et al., J. Mater. Chem. 16, 3369 (2006)

R. Elliott, Phys. Rev. 108, 1384 (1957)

C. Erginsoy, Phys. Rev. 79, 1013(1950)

S. Evans, N. C. Giles, L. E. Halliburton, and L. A. Kappers, J. Appl. Phys. 103, 043710 (2008)

M. Fox, Optical Properties of Solids, (Oxford, 2001)

N. Y. Garces, L. Wang, L. Bai, N. C. Giles, L. E. Halliburton, and G. Cantwell, Appl. Phys. Lett. 81, 622 (2002).

N. Y. Garces, L. Wang, L. Bai, N. C. Giles, L. E. Halliburton, G. Cantwell, and D. B. Eason, J. Appl. Phys. 94, 519 (2003).

J. G. E. Gardeniers, Z. M. Rittersma, and G. J. Burger, J. Appl. Phys. 83, 7844 (1998)

N. C. Giles, N. Y. Garces, Lijun Wang, and L. E. Halliburton, Invited paper, in Quantum Sensoring and Nanophotonic Devices, edited by M. Razeghi and G. J. Brown, Proc. Of SPIE Vol. 5359 , p.p. 267-277 (SPIE, Bellingham, WA, 2004)

N. C. Giles, Chunchuan Xu, M. J. Callahan, Buguo Wang, J. S. Neal, and L. A. Boatner, Appl. Phys. Lett. 89, 251906 (2006)

N. C. Giles, Yongquan Jiang, Xiaocheng Yang, S. M. Evans, and L. E. Halliburton, Mater. Res. Soc. Symp. Proc. Vol 957, 29 (2007)

W. Göpel, R. S. Bauer, and G. Hansson, Surf. Sci. 99, 138 (1980)

R. Gwin and R. B. Murray, Phys. Rev. 131, 501 (1963)

A. Hachigo, H. Nakahat, K. Higaki, S. Fujii, and S. Shikata, Appl. Phys. Lett. 65, 2556 (1994) 
L. E. Halliburton, Lijun Wang, Lihua Bai, N. Y. Garces, N. C. Giles, M. J. Callahan, and Buguo Wang, J. Appl. Phys. 96, 7168 (2004)

L. E. Halliburton, N. C. Giles, N. Y. Garces, Ming Luo, Chunchuan Xu, Liju Bai, and L. A. Boatner, Appl. Phys. Lett. 87, 172108 (2005)

D. W. Hamby, D. A. Lucca, M. J. Klopfstein, and G. Cantwell, J. Appl. Phys. 93, 3214 (2003)

R. Hauschild, H. Priller, M. Deecker, J. Brückner, H. Kalt, and C. Klingshirn, Phys. Stat. Sol. (c) 3, No. 4, 976 (2006)

Y. Hayashi, K. Watanabe, T. Jimbo, and M. Umeno, J. Appl. Phys. 37, 622(1999)

J. R. Haynes, Phys. Rev. 98, 1866 (1955)

J. R. Haynes, Phys. Rev. Lett. 4, 361 (1960)

E. C. Heltemes and H. L. Swinney, J. Appl. Phys. 38, 2387 (1967)

J. Hirsch, J. Phys. C: Solid State Phys. 12, 321 (1979)

N. H. Hill, and U. Waghmare, Phys. Rev. B 62, 8802 (2000)

R. Hofstadter, Phys. Rev. 75, 796 (1949)

M. Hong, X. Yang, D. Fredrick, D. M. DeVito, J. Howe, S. Yang, N. C. Giles, J. S. Neal, and Z. Munir, Journal of Materials Research (submitted July 2008)

J. J. Hopfield, D.G. Thomas, and M. Gershenzon, Phys. Rev. Lett. 10, 162 (1963)

K. Hümmer, Phys. Stat. Sol. 56, 249 (1973)

H. Ibach, Phys. Status Solidi 33, 257 (1969)

D.-S. Jiang, Y. Makita, K. Ploog, and H. J. Queisser, J. Appl. Phys. 53, 999 (1982)

Y. Jiang, N. C. Giles, and L. E. Halliburton, J. Appl. Phys. 101, 093706 (2007)

L. A. Kappers, O. R. Gilliam, S. M. Evans, L. E. Halliburton, and N. C. Giles, Nuclear Instruments and Methods in Physics Research B 266, 2953 (2008

H. Karzel, W. Potzel, M. Köfferlein, W. Schiessl, M. Steiner, U. Hiller, G. M. Kalvius, D. W. Mitchell, T. P. Das, P. Blaha, K. Schwarz, and M. P. Pasternak, Phys. Rev. B 53, 11425 (1996)

C. C. Klick and J. H. Schulman, Solid State Phys. 5, 97(1957)

C. F. Klingshirn, Semiconductor Optics, (Springer, Berlin, 1997) 
I. B. Kobiakov, Solid State Commun. 35, 305 (1980)

A. F. Kohan, G. Ceder, D. Morgan, and C. G. van de Walle, Phys. Rev. B 61, 15019 (2000)

R. Kuhnert and R. Helbig, J. Lumin. 26, 203 (1981)

Landolt-Börnstein, in Intrinsic Properties of Group IV Elements and III-V, II-VI and I-VII Compounds, edited by O. Madelung and M. Schulz, New Series, Group III, Vol. 22a (Springer, Berlin, 1987)

W. Lehmann, J. Electrochem. Soc. 112, 1150 (1965)

W. Lehmann, Solid-State Electronics 9, 1107 (1966)

Y. Liu, J. Electron. Mater. 29, 69 (2000)

D. C. Look, Electrical Characterization of GaAs Materials and Devices (Wiley, New York, 1989), Chapter 1.

D. C. Look, D. C. Reynolds, W. Kim, Ö. Aktas, A. Botchkarev, A. Salvador, and H. Morkoç, J. Appl. Phys. 80, 2960 (1996)

D. C. Look, D. C. Reynolds, J. R. Sizelove, R. L. Jones, C. W. Litton, G. Cantwell, and W. C. Harsch, Solid State Commun. 105, 399 (1998)

D. C. Look, D. C. Reynolds, C. W. Litton, R. L. Jones, D. B. Eason, and G. Cantwell, Appl. Phys. Lett. 81, 1830 (2002)

D. C. Look, R. L. Jones, J. R. Sizelove, N. Y. Garces, N. C. Giles and L. E. Halliburton, Phys. Stat. Sol. (a) 195 (1), 171 (2003)

D. C. Look, G. M. Renlund, R. H. Burgener II, and J. R. Sizelove, Appl. Phys. Lett. 85, 5269 (2004)

D. C. Look, B. Claflin, Ya. I. Alivov, and S. J. Park, Phys. Stat. Sol. A 201, 2203 (2004)

D. C. Look, Semicond. Sci. Technol. 20, S55-S61 (2005)

M. Lorenz, R. Johne, T. Nobis, H. Hochmuth, J. Lenzner, M. Grundmann, H. P. D. Schenk, S. I. Borenstain, A. Schön, C. Bekeny, T. Voss, and J. Gutowski, Appl. Phys. Lett. 89, 243510 (2006)

D. W. Ma, Z. Z. Ye, and L. L. Chen, Phys. Status Solidi A 201, 2929 (2004))

G. D. Mahan, in Polarons in Ionic Crystals and Polar Semiconductors, edited by J. T. Devreese, (N.H P\&C, 1972) Chapter VI

T. Makino, A. Tsukazaki, A. Ohtomo, M. Kawasaki and H. Koinuma, Jpn. J. Appl. Phys. 45 (8A) 6346 (2006) 
A. Manoogian and J. C. Woolley, Can. J. Phys. 62, 285 (1984)

J. J. Markham, F-centers in Alkali Halides, (Academic, New York,1966) Chapter I

B. K. Meyer, H. Alves, D. M. Hofmann, W. Kriefseis, D. Forster, F. Bertram, J. Christen, A. Hoffmann, M. Straßburg, M. Dworzak, U. Haboeck, and A. V. Rodina, Phys. Stat. Sol. (b) 241, 231 (2004)

J. Nause, III-Vs Review 12, 28 (1999)

J. Nause and B. Nemeth, Semicond. Sci. Technol. 20, S45-S48 (2005)

J. S. Neal, N. C. Giles, X. Yang, R. A. Wall, K. B. Ucer, R. T. Williams, D. J. Wisniewski, L. A. Boatner, V. Rengarajan, J. Nause, and B. Nemeth, IEEE Trans. Nucl. Sci. 55, 1397 (2008)

J. S. Neal, D. J. Wisniewski, L. A. Boatner, N. C. Giles, X. Yang, V. Rengarajan, J. Nause, and B. Nemeth, presentation at IEEE -9th International Conference on Inorganic Scintillators and their Applications (SCINT 2007), Winston-Selem, NC, (2007)

J. S. Neal, D. M. DeVito, M. Hong, B. Kesanli, J. O. Ramey, B. L. Armstrong, X. Yang, N. C. Giles, J. Y. Howe, D. J. Wisniewski, M. Wisniewska, Z. A. Munir, and L. A. Boatner, IEEE Transactions of Nuclear Sciences (submitted June 2008)

H. M. Ng, D. Doppalapudi, T. D. Moustakas, N. G. Weimann and L. F. Eastman, Appl. Phys. Lett. 73, 821(1998)

A. Ohtomo, M. Kawasaki, T. Koida, K. Masubuchi, and H. Koinuma, Appl. Phys. Lett. 72, 2466 (1998)

A. Ohtomo, K. Tamura, K. Saikusa, K. Takahashi, T. Makino, Y. Segawa, H. Koinuma, and M. Kawasaki, Appl. Phys. Lett. 75, 2635 (1999)

Ü. Özgür, Ya. I. Alivov, C. Liu, A. Teke, M. A. Reshchikov, S. Doğan, V. Avrutin,S.-J. Cho, and H. Morkoç, J. Appl. Phys. 98, 041301 (2005).

J. I. Pankove, Optical Processes in Semiconductors (Dover, New York, 1971)

C. H. Park, S. B. Zhang, and S.-H. Wei, Phys. Rev. B 66, 073202 (2002)

G. L. Pearson and J. Bardeen, Phys. Rev. 75, 865 (1949)

E. H. Putley, The Hall Effect and Semiconductor Physics (Dover, New York, 1968), p.146)

D. L. Rode, Phys. Rev. B 2, 4036 (1970)

D. L. Rode, in Semiconductors and Semimetals, edited by R. K. Willardson and A. C. Beer (Academic Press, New York, 1975) Vol 10, Chapter 1 
P. A. Rodnyi, Physical Process in Inorganic Scintillators (CRC Press, Boca Raton, FL, 1997)

E. Rutherford, J. Chadwich, and C. D. Ellis, Radiations from Radioactive Substances

(Cambridge University Press, Cambrudge, 1930)

Y. R. Ryu, T. S. Lee, and H. W. White, Appl. Phys. Lett. 83, 87 (2003)

M. Schirra, R. Schneider, A. Reiser, G. M. Prinz, M. Feneberg, J. Biskupik, U. Kaiser, C. E. Krill, K. Thonke, and R. Sauer, Phys. Rev. B 77, 125215 (2008)

H.-J. Schulz and M. Thiede, Phys. Rev. B 35, 18 (1987)

B. Segall and G. D. Mahan, Phys. Rev. 171, 935 (1968), as referred to by H. B. Bebb and E. W. Williams, in "Semiconductors and Semimetals" ed. by R. K. Willardson and A. C. Beer, (Academic, New York, 1972), Vol 8, Page 295

G. A. Shi, M. Stavola, S. J. Pearton, M. Thieme, E. V. Lavrov, and J. Weber, Phys. Rev. B $72,195211(2005)$

P. J. Simpson, R. Tjossem, A. W. Hunt, K. G. Lynn, and V. Munné, Nuclear Instruments and Methods in Physics Research A 505, 82 (2003)

A. V. Singh, R. M. Mehra, N. Buthrath, A. Wakahara, and A. Yoshida, J. Appl. Phys. 90, $5661(2001)$

M. Suscavage, M. Harris, D. Bliss, P. Yip, S.-Q. Wang, D. Schwall, L. Bouthillette, J. Bailey, M. Callahan, D. C. Look, D. C. Reynolds, R. L. Jones, and C. W. Litton, MRS Internet J. Nitride Semicond. Res. 4S1 G3.40 (1999)

D.G. Thomas, M. Gershenzon, and F. A. Trumbore, Phys. Rev. 133, A269 (1964)

K. Thonke, Th. Gruber, N. Teofilov, R. Schönfelder, A. Waag, R. Sauer, Physica B 308-310, $945(2001)$

L. J. van der Pauw, Philips Res. Rep. 13, 1-9 (1958)

C. Van de Walle, Physica B 308-310, 899 (2001)

J. A. Van Vechten, and T. K.Bergstresser, Phys. Rev. B 1, 3351 (1970)

Y. P. Varshni, Physica, 34, 149 (1967)

R. D. Vispute, V. Talyansky, S. Choopun, R. P. Sharma, T. Venkatesan, M. He, X. Tang, J. B. Halpern, M. G. Spencer, Y. X. Li, L. G. Saalamanca-Riba, A. A. Iliadis, and K. A. Jones, Appl. Phys. Lett. 73, 348 (1998)

P. Wagner und R. Helbig, J. Phys. Chem. Solids 35, 327(1974)

J. P. Walter, R. R. L. Zucca, M. L. Cphen, and Y. R. Shen, Phys. Rev. Lett. 24, 102 (1970) 
L. Wang and N. C. Giles, J. Appl. Phys. 94, 973 (2003)

L. Wang and N. C. Giles, Appl. Phys. Lett. 84, 3049 (2004)

J. Wilkinson, K. B. ucer, and R. T. Williams, radiation measurements 38, 501 (2004)

J. Wilkinson, K. B. ucer, and R. T. Williams, Nuclear Instruments and Methods in Physics Research A 537, 66 (2005)

H. H. Woodbury and M. Aven, Phys. Rev. B 8, 5195(1974)

P. Y. Yu, Fundamentals of Semiconductors, (Springer, New York, 2001)

Y. Yu, S. Nam, K. Lee, Y. Choi, and B. O, J. Appl. Phys. 90, 807 (2001)

F. Zhuge, L. P. Zhu, Z. Z> Ye, D. W. Ma, J. G. Lu, J. Y. Huang, F. Z. Wang, Z. G. Ji, and S.

B. Zhang, Appl. Phys. Lett. 87, 092103 (2005)

J. D. Zook and R. N. Dexter, Phys. Rev. 129 (5), 1980 (1963)

J. D. Zook, Phys. Rev. 136 (3A), A869 (1964) 


\section{Curriculum Vitae}

\section{Xiaocheng Yang}

Department of Physics

P. O. Box 6315

West Virginia University

Morgantown, WV 26506
Tel (w) (304) 293-3422, ext. 1445

Cell: (304) 685-0871

Email: xyang3@mix.wvu.edu

Personal: F-1 student visa; Citizenship: People's Republic of China

\section{Education:}

September 2008: Ph.D. in condensed matter physics

Department of Physics, West Virginia University, West Virginia, USA

Dissertation: Electrical and Optical Properties of Zinc Oxide for

Scintillator Applications

May, 2003: Masters of Science in Optical Physics

Department of Physics, Capital Normal University, Beijing, China

Thesis: Raman and Surface-Enhanced Raman Spectroscopic Study on 60\&70 Fullerene Organic Solutions and Thin Films

July, 1990: Bachelor of Science in Physics

Dept. of Technological Physics, Peking University, Beijing, China

Thesis: Property Examination of HP-Ge spectrometer: Calibration of Energy and Absolute Efficiency

\section{Employment:}

2005-present: Graduate Research Assistant, doctoral research,

Advisor: Dr. Nancy Giles

Department of Physics, West Virginia University, West Virginia, USA

2003-2005: Graduate Teaching Assistant

Department of Physics, West Virginia University, West Virginia, USA

2000-2003: Graduate Research Assistant

Advisor: Dr. Yan Fang

Beijing Key Lab for Nanophotonics and Nanostructure, Department of

Physics, Capital Normal University, Beijing, China

1990-2000 Instructor/Lecturer of Physics

Department of Physics, Pingliang Normal School, Gansu, China

\section{Professional membership:}

Member, American Physical Society

Member, Materials Research Society 


\section{Publications:}

"Development of ZnO-based polycrystalline ceramic scintillators for use as alphaparticle detectors," John S. Neal, David M. DeVito, Mei Hong, Banu Kesanli, Joanne O. Ramey, Beth L. Armstrong, Xiaocheng Yang, Nancy C. Giles, Jane Y. Howe, Dariusz J. Wisniewski, Monika Wisniewska, Zuhair A. Munir, and Lynn A. Boatner, IEEE Transactions of Nuclear Sciences (in press)

"Intrinsic electron mobilities in CdSe, CdS, ZnO, and ZnS and analysis of temperature-dependent Hall measurements," Xiaocheng Yang, Chunchuan $\mathrm{Xu}$, and Nancy. C. Giles, Journal of Applied Physics, (in press)

"A simple aqueous metathesis reaction yields new lanthanide monothiophosphates," Nathan J. Takas, Lauren E Slomka, Xiaocheng Yang, Nancy C. Giles, and Jennifer A Aitken, Journal of Solid State Chemistry (submitted April 2008)

"Evaluation of Melt-Grown, ZnO Single Crystals for Use as Alpha-Particle Detectors," John S. Neal, Nancy C. Giles, Xiaocheng Yang, R. Andrew Wall, K. Burak Ucer, Richard T. Williams, Dariusz J. Wisniewski, Lynn A. Boatner, Varathajan Rengarajan, Jeff Nause, and Bill Nemeth, IEEE Transactions of Nuclear Sciences, Vol. 55, No. 3, 1397-1403 (2008)

"Role of Neutral Impurity Scattering in the Analysis of Hall Data from ZnO and Other II-VI Materials," Xiaocheng Yang, Chunchuan Xu, and N. C. Giles, In Zinc Oxide and Related materials -2007 , ed. by D. P. Norton, C. Jagadish, I. Buyanova, and GC. Yi, (Mater. Res. Soc. Symp. Proc. Volume 1035E, L04-07, Warrendale, PA, 2008)

"Persistent photoinduced changes in charge states of donors and acceptors in hydrothermally grown ZnO," N. C. Giles, Yongquan Jiang, Xiaocheng Yang, S. M. Evans, L. E. Halliburton, Mater. Res. Soc. Symp. Proc. 2007 Materials Research Society, 957 (Zinc Oxide and related Materials) 29-34 (2007)

"Optical and Electrical Characterization of Cadmium Selenide Doped with Cobalt," Ming Luo, Yongquan Jiang, Chunchuan Xu, Xiaocheng Yang, A. Burger, N. C. Giles, J. Phys. Chem. Solids, Vol. 67, 2596-2602 (2006)

"Surface-Enhanced Raman Scattering of $\mathrm{C}_{60}$ and $\mathrm{C}_{70}$ in Gold Hydrosols," X.-C. Yang, and Y. Fang, J. Phys. Chem. B, Vol. 107, Number 37, (2003) 10100-10103

\section{Presentations:}

"Electrical and Optical Properties of Zinc Oxide for Scintillator Applications", Xiaocheng Yang, PhD dissertation defense, Department of Physics, West Virginia University, Sep. 9, 2008 
"Absorption and Photoluminescence from Highly Conductive N-type ZnO," Xiaocheng Yang, John S. Neal, Lynn A. Boatner, and N. C. Giles, 2008 Materials Research Society Meeting, Boston, MA, Dec 1-5, 2008 (abstract submitted June 2008)

"Development of ZnO-based polycrystalline ceramic scintillators for use as alphaparticle detectors," John S. Neal, David M. DeVito, Mei Hong, Banu Kesanli, Joanne O Ramey, Beth L. Armstrong, Xiaocheng Yang, Nancy C. Giles, Jane Y. Howe, Dariusz J. Wisniewski, Monika Wisniewska, Zuhair A. Munir, and Lynn A. Boatner, 2008 Symposium on Radiation Measurements and Applications (SORMA West 2008), Berkerley, CA, June-2-5, 2008

"Role of Neutral impurity Scattering in the Analysis of Hall Data from ZnO and Other II-VI Materials," Xiaocheng Yang, Chunchuan Xu, and N. C. Giles, 2007 Materials Research Society Meeting, Boston, MA, Nov. 27, 2007

"Photoluminescence Study of ZnO:Ga for Scintillator Applications," Xiaocheng Yang, and N. C. Giles, J. S. Neal, B. Kesanli, J. Y. Howe, J. O. Ramey, B. L. Armstrong, and L. A. Boatner, 2007 Workshop on Physics and Chemistry of II-VI Materials, Baltimore, MD, Oct. 30, 2007

Evaluation of melt-grown, ZnO single crystals for use as a-particle detectors." John S. Neal, Dariusz J. Wisniewski, Lynn A. Boatner, Nancy C. Giles, Xiaocheng Yang, Varathajan Rengarajan, Jeff Nause, and Bill Nemeth, IEEE -9th International Conference on Inorganic Scintillators and their Applications (SCINT 2007), WinstonSelem, NC, June 4-8, 2007

"Role of Neutral impurity Scattering in the Analysis of Hall Data from ZnO," Xiaocheng Yang, Chunchuan Xu, and Nancy Giles, 2007 American Physical Society Meeting, Denver, CO, Mar. 8, 2007 